\title{
PROTEIN-BASED INJECTABLE HYDROGELS TOWARDS THE REGENERATION OF ARTICULAR CARTILAGE
}

\author{
Ph. D. Thesis by \\ Sara Poveda Reyes \\ to obtain the degree of Doctor \\ at the Universitat Politècnica de València \\ Valencia (Spain), January 2016
}

Thesis supervisor:

Dr. Gloria Gallego Ferrer 

A mis seres queridos,

con ellos todo es posible 

"The future belongs to those who believe in the beauty of their dreams" Eleanor Roosevelt 



\section{Acknowledgements}

After reaching this point I would like to thank everybody that in one way or another has helped me through these four years of work.

First of all, I gratefully acknowledge the financial support from the Spanish Ministry of Economy and Competitiveness through the DPI2010-20399-C04-03 and MAT2013-46467-C4-1-R projects, my FPI fellowship BES-2011-046144 and the EEBB-I-13-06179 and EEBB-I-1408725 research stay grants.

I would especially like to thank my thesis supervisor, Dr. Gloria Gallego Ferrer, for helping me in everything I needed through these four years, for giving me this opportunity and for all her efforts in my assistance. Also thanks to José Luis Gómez Ribelles for his valuable help and brilliant ideas.

Thanks to the staff of the Microscopy Service at the Universitat Politècnica de València, for their guidance and help during the SEM sessions.

I would also like to express my gratitude to my supervisors during my stays at other Research Centers. Thanks to Prof. Ulrica Edlund, who during my stay at the Fiber and Polymer Technology Department (KTH) in Stockholm, helped me with everything I could need and to all the people in the center (Laleh, Anas, Soheil, Bekir, Parmida, Robertus, Arturo, etc.) that helped me in the lab and made my stay easier. Thanks to Prof. Manuel Salmerón Sánchez at the Microenvironments for Medicine group (MiMe) (University of Glasgow) for giving me the opportunity to learn the basics of cell culture in his lab, his guidance and valuable help. I would also like to thank Dr. Vladimira Moulisova for teaching me everything I know of cell biology work and being so patient and friendly. And to all the people of the group (Marco, Sara, Eleni, Mark, Alex, Jake, Elie, Frankie, etc.), as they say "People make Glasgow" and they made me feel at home. 
I also want to express my gratitude to Luis Quintanilla and the BIOFORGE Group (University of Valladolid) for the help with the rheology measurements and their contribution to this work.

I want to thank as well the Group of Structural Mechanics and Materials Modelling (GEMM) (University of Zaragoza) for their help with the permeability and compression measurements of the IPNs.

I cannot forget all the people at the Center for Biomaterials and Tissue Engineering: to Laura Teruel, for being so patient with everyone, so helpful and keeping the lab in a perfect state, and all the lecturers for their help and advice, especially to Ana Vallés and Manuel Monleón, who introduced me to the wonderful field of Tissue Engineering.

I would like to especially thank Tatiana C. Gamboa, who guided me in my first years in the lab. To Suhail, Rocío, Marta, Félix, Petra, Leonardo, Rebeca and Esther, I helped them in their first steps in the lab but I learnt a lot from them and they helped me a lot, both professional and personally. Also thanks to Alex Rodrigo who helped me a lot in my first cell cultures even if he had a million things to do.

Thanks to all the people who have been in the "Sala de Reuniones" office: Manu, María, Line, Amparo G, Roberto, Guillermo, Álvaro, Laia, Rubén, etc., thanks for the break times, your laughs, your help and being the way you are.

Special thanks to my friends, they gave me support through all these years, with them life is better.

Finally, I would like to thank my family for supporting me in everything I wanted to do, even if that meant I could not be with them all the time I wanted. Without them this would not have been possible.

Special thanks to Nacho, for being so understanding and always being by my side. 


\section{Table of contents}

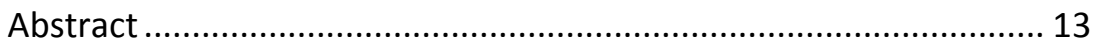

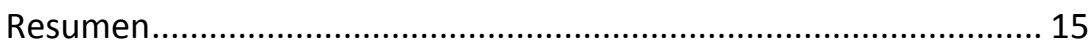

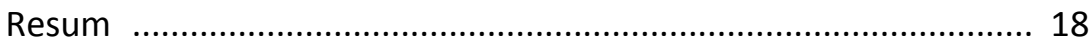

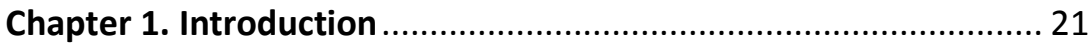

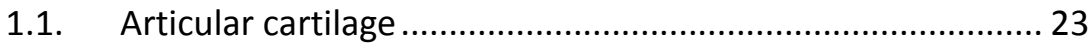

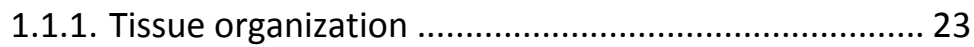

1.1.2. Articular cartilage properties ..................................... 28

1.1.3. Articular cartilage injuries .......................................... 33

1.1.4. Articular cartilage repair therapies ............................. 36

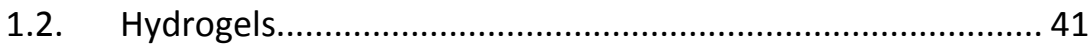

1.3. Gelatin-Hyaluronic acid hydrogels ........................................ 45

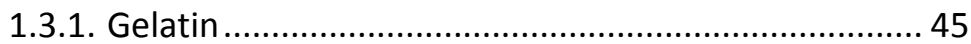

1.3.2. Hyaluronic acid.......................................................... 47

1.3.3. Extracelular-matrix inspired hydrogels of gelatin and hyaluronic acid ................................................ 48

1.4. Injectable enzymatically crosslinked hydrogels of gelatin

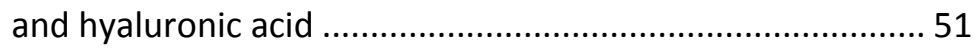

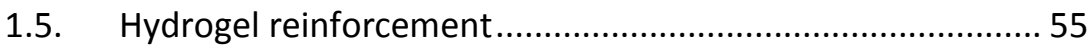

1.5.1. Hydrogels reinforced by Interpenetrating Polymer

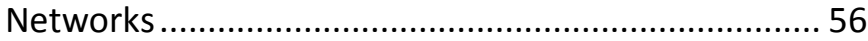

1.5.2. Hydrogels reinforced by combination with microfibers ................................................................ 58

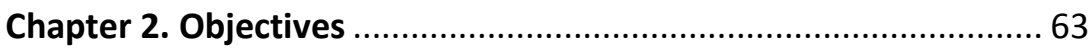

Chapter 3. Injectable gelatin-fiber hydrogels ................................ 67 
3.2. Interaction of two different types of PLLA fibers with gelatin. Two routes for short fiber production.

3.2.1. Materials and methods ............................................... 71

3.2.2. Results and Discussion ................................................ 79

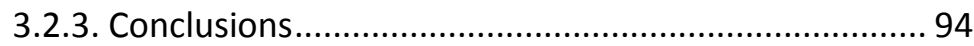

3.3. Injectable Gelatin-fiber Composites with Improved

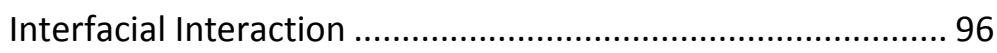

3.3.1. Materials and Methods ............................................... 96

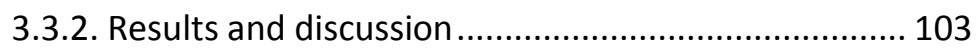

3.3.3. Conclusions ............................................................. 121

Chapter 4. ECM inspired injectable Gelatin-Hyaluronic acid

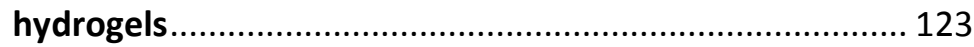

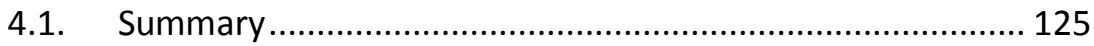

4.2. Injectable gelatin-hyaluronic acid hydrogels with high molecular weight HA ...................................................... 126

4.2.1. Materials and methods ............................................ 126

4.2.2. Results and discussion .............................................. 130

4.2.3. Conclusions................................................................ 139

4.3. Injectable gelatin-hyaluronic acid hydrogels with low molecular weight hyaluronic acid. Physico-chemical

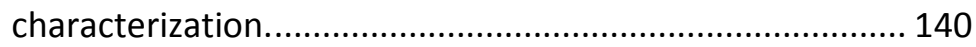

4.3.1. Materials and methods ............................................ 140

4.3.2. Results and discussion ............................................... 143

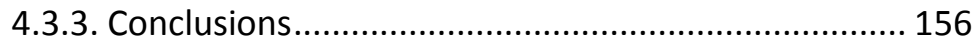

4.4. $\mathrm{C} 2 \mathrm{C} 12$ differentiation in gelatin/hyaluronic acid injectable hydrogels 
4.4.2. Results and discussion ............................................... 160

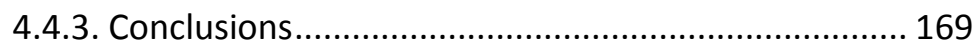

4.5. Condrogenic differentiation of BM-hMSCs in gelatinhyaluronic acid injectable hydrogels .................................. 171

4.5.1. Materials and Methods ............................................ 171

4.5.2. Results and discussion ............................................... 174

4.5.3. Conclusions .................................................................. 198

Chapter 5. Synthetic IPNs as ex vivo platforms for cartilage regeneration ............................................................... 199

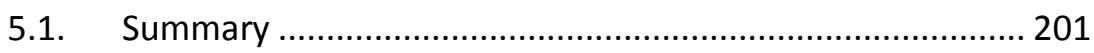

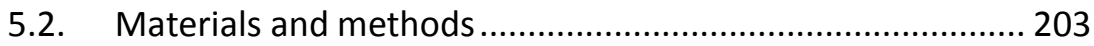

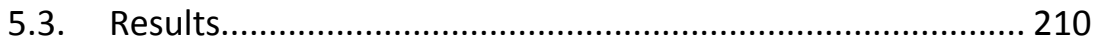

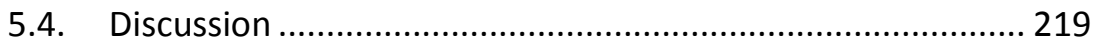

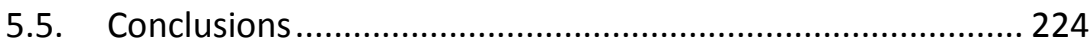

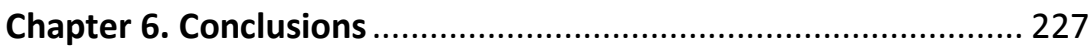

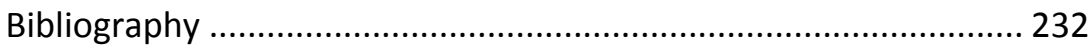

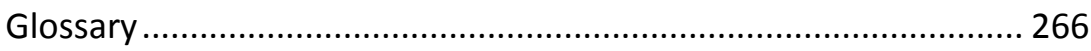

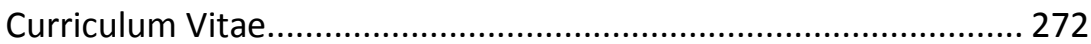





\section{Abstract}

Articular cartilage is a tissue with low capacity for self-restoration due to its avascularity and low cell population. It is located on the surface of the subchondral bone covering the diarthrodial joints. Degeneration of articular cartilage can appear in athletes, in people with genetic degenerative processes (osteoarthritis or rheumatoid arthritis) or due to a trauma; what produces pain, difficulties in mobility and progressive degeneration that finally leads to joint failure. Self-restoration is only produced when the defect reaches the subchondral bone and bone marrow mesenchymal stem cells (MSCs) invade the defect. However, this new formed tissue is a fibrocartilaginous type cartilage and not a hyaline cartilage, which finally leads to degeneration. Transplantation of autologous chondrocytes has been proposed to regenerate articular cartilage but this therapy fails mainly due to the absence of a material support (scaffold) for the adequate stimulation of cells. Matrix-induced autologous chondrocyte implantation uses a collagen hydrogel as scaffold for chondrocytes; however, it does not have the adequate mechanical properties, does not provide the biological cues for cells and regenerated tissue is not articular cartilage but fibrocartilage. Different approaches have been done until now in order to obtain a scaffold that better mimics articular cartilage properties and composition. Hydrogels are a good option as they retain high amounts of water, in a similar way to the natural tissue, and can closely mimic the composition of natural tissue by the combination of natural derived hydrogels. Their three-dimensionality plays a critical role in articular cartilage tissue engineering to maintain chondrocyte function, since monolayer culture of chondrocytes makes them dedifferentiate towards a fibroblast-like phenotype secreting fibrocartilage.

Recently, injectable hydrogels have attracted attention for the tissue engineering of articular cartilage due to their ability to encapsulate cells, injectability in the injury with minimal invasive surgeries and 
adaptability to the shape of the defect. Following this new approach we aimed at synthesizing two new families of injectable hydrogels based on the natural protein gelatin for the tissue engineering of articular cartilage.

The first series of materials consisted on the combination of injectable gelatin with loose reinforcing polymeric microfibers to obtain injectable composites with improved mechanical properties. Our results demonstrate that there is an influence of the shape and distribution of the fibers in the mechanical properties of the composite. More importantly bad fiber-matrix interaction is not able to reinforce the hydrogel. Due to this, our composites were optimized by improving matrix-fiber interaction through a hydrophilic grafting onto the microfibers, with very successful results.

The second series of materials were inspired in the extracellular matrix of articular cartilage and consisted of injectable mixtures of gelatin and hyaluronic acid. Gelatin molecules in the mixtures provided integrin adhesion sites to cells, and hyaluronic acid increased the mechanical properties of gelatin. This combination demonstrated ability for the differentiation of MSCs towards the chondrocytic lineage and makes these materials very good candidates for the regeneration of articular cartilage.

The last part of this thesis is dedicated to the synthesis of a nonbiodegradable material with mechanical properties, swelling and permeability similar to cartilage. This material intends to be used as a platform in a bioreactor in which the typical loads of the joint are simulated, so that the hydrogels or scaffolds would fit in the recesses in the platform. The function of the platform is to simulate the effect of the surrounding tissue on the scaffold after implantation and could reduce animal experimentation by simulating in vivo conditions. 


\section{Resumen}

El cartílago articular es un tejido con baja capacidad de autoreparación debido a su avascularidad y baja población celular. Se encuentra en la superficie del hueso subcondral cubriendo las articulaciones diartrodiales. La degeneración del cartílago articular puede aparecer en atletas, en personas con procesos genéticos degenerativos (osteoartritis y artritis reumatoide) o debido a un trauma; lo que produce dolor, dificultades en la movilidad y degeneración progresiva que finalmente lleva al fallo de la articulación. La auto-reparación sólo se produce cuando el defecto alcanza el hueso subcondral y las células madre (MSCs) de la médula ósea invaden el defecto. Sin embargo, este nuevo tejido formado es un cartílago de tipo fibrocartilaginoso y no un cartílago hialino, el cual finalmente lleva a la degeneración. El trasplante de condrocitos autólogos ha sido propuesto para regenerar el cartílago articular pero esta terapia falla principalmente por la ausencia de un material soporte (scaffold) que estimule adecuadamente a las células. El implante de condrocitos autólogos mediante una matriz utiliza un hidrogel de colágeno como scaffold para los condrocitos; sin embargo, éste no tiene las propiedades mecánicas apropiadas, no proporciona las señales biológicas a las células y el tejido regenerado no es cartílago articular sino fibrocartílago. Diferentes enfoques han sido realizados hasta ahora para obtener un scaffold que mimetice mejor las propiedades y la composición del cartílago articular. Los hidrogeles son una buena opción ya que retienen elevadas cantidades de agua, de forma similar al tejido natural, y pueden imitar de cerca la composición del tejido natural mediante la combinación de derivados de hidrogeles naturales. Su tridimensionalidad juega un papel crítico en la ingeniería de tejidos del cartílago articular para mantener la función de los condrocitos, ya que el cultivo en monocapa de los condrocitos hace que éstos 
desdiferencien hacia un fenotipo similar al fibroblasto secretando fibrocartílago.

Recientemente, los hidrogeles inyectables han acaparado la atención en la ingeniería tisular de cartílago articular debido a su capacidad para encapsular células, su inyectabilidad en el daño con cirugías mínimamente invasivas y su adaptabilidad a la forma del defecto. Siguiendo este nuevo enfoque hemos sintetizado dos nuevas familias de hidrogeles inyectables basados en la proteína natural gelatina para la ingeniería tisular del cartílago articular.

La primera serie de materiales ha consistido en la combinación de gelatina inyectable con microfibras poliméricas sueltas de refuerzo para obtener composites inyectables con propiedades mecánicas mejoradas. Nuestros resultados demuestran que hay una influencia de la forma y la distribución de las fibras en las propiedades mecánicas del composite. Más importantemente, la mala interacción entre las fibras y la matriz no es capaz de reforzar el hidrogel. Debido a esto, nuestros composites han sido optimizados mediante la mejora de la interacción fibra-matriz a través de un injerto hidrófilo sobre las microfibras, con resultados muy exitosos.

La segunda serie de materiales se ha inspirado en la matriz extracelular del cartílago articular y ha consistido en mezclas inyectables de gelatina y ácido hialurónico. Las moléculas de gelatina en las mezclas proporcionan los dominios de adhesión mediante integrinas a las células, y el ácido hialurónico aumenta las propiedades mecánicas de la gelatina. Esta combinación ha demostrado la habilidad para la diferenciación de MSCs hacia el linaje condrocítico y convierte a estos materiales en muy buenos candidatos para la regeneración del cartílago articular.

La última parte de esta tesis está dedicada a la síntesis de un material no biodegradable con propiedades mecánicas, hinchado y permeabilidad similar al cartílago. Este material pretende ser empleado como plataforma en un biorreactor en el que se simulan las cargas típicas de las articulaciones, de forma que los hidrogeles o 
scaffolds encajarían en los huecos de la plataforma. La función de la plataforma es simular el efecto del tejido circundante en el scaffold después de su implantación y podría reducir la experimentación animal mediante la simulación de las condiciones in vivo. 


\section{Resum}

El cartílag articular es un teixit amb baixa capacitat d'auto-reparació deguda a la seua avascularitat i baixa població cel-lular. Es troba en la superfície de l'ós subcondral cobrint les articulacions diartroidals. La degeneració del cartílag articular pot aparèixer en atletes, en persones amb processos genètics degeneratius (osteoartritis $i$ artritis reumatoide) o degut a un trauma; produeix dolor, dificultats a la mobilitat i degeneració progressiva que finalment porta a la fallida de I'articulació. L'auto-reparació solament es produeix quan el defecte arriba fins a l'ós subcondral i les cèl-lules mare de la medul.la òssia (MSCs) envaeixen el defecte. No obstant això, aquest nou teixit format es un cartílag de tipus fibrocartilaginós i no un cartílag hialí, el qual finalment porta a la degeneració. El transplantament de condròcits autòlegs ha sigut proposat per a regenerar el cartílag articular però aquesta teràpia falla principalment per l'absència d'un material de suport (scaffold) que estimuli adequadament a les cèl-lules. L'implant de condròcits autòlegs en una matriu utilitza un hidrogel de col-lagen com scaffold per als condròcits; no obstant això, aquest no té les propietats mecàniques apropiades, no proporciona les senyals biològiques a les cèl-lules i el teixit regenerat no és cartílag articular sinó fibrocartílag. Diferents enfocs han sigut realitzats fins ara per a obtenir un scaffold que mimetitzi millor les propietats i la composició del cartílag articular. Els hidrogels son una bona opció ja que retenen elevades quantitats d'aigua, de forma similar al teixit natural, i poden imitar acuradament la composició del teixit natural mitjançant la combinació de derivats d'hidrogels naturals. La seua tridimensionalitat juga un paper crític a l'enginyeria tissular del cartílag articular per a mantenir la funció dels condròcits, ja que el cultiu en monocapa dels condròcits fa que aquests desdiferencien cap a un fenotip similar al fibroblàstic secretant fibrocartílag.

Recentment, els hidrogels injectables han acaparat I'atenció de l'enginyeria tissular de cartílag articular degut a la seua capacitat per 
a encapsular cèl-lules, la seua injectabilitat en el dany amb cirurgies mínimament invasives i la seua adaptabilitat a la forma del defecte. Seguint aquesta nova aproximació hem sintetitzat dues noves famílies d'hidrogels injectables basats en la proteïna natural gelatina per a l'enginyeria tissular del cartílag articular.

La primera sèrie de materials ha consistit en la combinació de gelatina injectable amb microfibres polimèriques soltes de reforç per a obtenir compòsits injectables amb propietats mecàniques millorades. Els nostres resultats demostren que hi ha una influència de la forma i la distribució de les fibres en les propietats mecàniques del compòsit. Més importantment, la mala interacció entre les fibres $i$ la matriu no és capaç de reforçar l'hidrogel. Degut a això, els nostres compòsits han segut optimitzats mitjançant la millora de la interacció fibra-matriu a traves d'un empelt hidròfil sobre les fibres, amb resultats molt exitosos.

La segona sèrie de materials està inspirada en la matriu extracel-lular del cartílag articular i ha consistit en mescles injectables de gelatina $\mathrm{i}$ àcid hialurònic. Les molècules de gelatina a les mescles proporcionen els dominis d'adhesió mitjançant integrines a les cèl-lules, i l'àcid hialurònic augmenta les propietats mecàniques de la gelatina. Esta combinació ha demostrat I'habilitat per a la diferenciació de MSCs cap al llinatge condrocític i converteix a aquests materials en molt bons candidats per a la regeneració del cartílag articular.

L'última part d'aquesta tesi és dedicada a la síntesi d'un material no biodegradable amb propietats mecàniques, inflat i permeabilitat similar al cartílag. Aquest material pretén ser utilitzat com a plataforma a un bioreactor que simula les cargues típiques de les articulacions, de manera que els hidrogels o scaffolds encaixarien als buits de la plataforma. La funció de la plataforma és simular l'efecte del teixit circumdant al scaffold després de la seua implantació i podria reduir l'experimentació animal mitjançant la simulació de les condicions in vivo. 



\section{Chapter 1}

\section{Introduction}





\subsection{Articular cartilage}

Articular hyaline cartilage is a type of tissue located on the surface of the subchondral bone covering the diarthrodial joints. Its principal functions are to avoid friction between the articular surfaces of the joint in movement and to absorb, dissipate and distribute loads to the subchondral bone [2]. This human articular cartilage is normally around 2-4 $\mathrm{mm}$ thick [3].

\subsubsection{Tissue organization}

Articular cartilage can be considered a complex multiphasic tissue with both fluid and solid phases. The fluid phase is composed of water and dissolved electrolytes (60-85\% of wet weight), and the solid phase is formed by the extracellular matrix (ECM), which has collagen $(10-30 \%$ of the wet weight), proteoglycans $(3-10 \%$ of wet weight) and some glycoproteins and lipids [4].

Type II collagen forms $90 \%$ of the macrofibrillar collagen network [4]. This collagen is in the form of fibrils composed of three $\alpha_{1}$ (II) chains, forming a triple helix with amino and carboxyl groups at each end. Intra and intermolecular bonds are formed between the lysine residues present in the collagen chains to compose the fibril. Type IX collagen represents $2 \%$ of the collagen fibril and is located on its surface in an antiparallel direction (see Figure 1-1). Collagen Type XI is also present within the fibril and on its surface, and its main functions are fibril self-assembly and limiting its lateral growth. The proteoglycan decorin is bonded to the collagen fibril, reducing its final diameter [2]. The percentage of collagen decreases with distance from the articular surface [5].

Proteoglycans and Type II collagen are some of the principal components of articular cartilage. Proteoglycan concentration varies inversely with collagen content. Lower concentrations of 
proteoglycan are found on the surface and higher in the deeper zones near the subchondral bone [2], [5]. They are composed of a protein core to which glycosaminoglycans (GAGs), chondroitin sulfate (CS) and keratan sulfate (KS) are covalently attached to a serine residue in the protein (see Figure 1-1). This core protein is bounded by the amino terminal G1 domain and the carboxy terminal G3 domain. The $\mathrm{G} 1$ domain binds to hyaluronic acid (HA) in a bond that is stabilized by a link protein, which has a similar structure to the G1 domain [2], [6]. Proteoglycans do not crosslink the hyaluronic acid chains but only bind to one binding site [2], [5]. The majority of proteoglycans present in articular cartilage are aggrecans [6], which bind to hyaluronic acid and form aggregates of $2 \times 10^{5} \mathrm{kDa}$ [4]. The main characteristic of chondroitin sulfate and keratan sulfate is their high capacity for hydration, which is restricted by the presence of the collagen fibrillary network, conferring good compressive stiffness to articular cartilage [2]. The high swelling capacity is due to the presence of repeating sulfate and carboxylate groups along the proteoglycans chains, which become negatively charged in aqueous solutions. The high concentration of negatively charged proteoglycans applies strong swelling pressure and tensile forces to the collagen network [4], [7].

The ECM is synthesized and maintained by the specialized cells known as chondrocytes, which compose $10 \%$ of the wet weight of articular cartilage [4] or $2 \%$ of the total volume in human adults [2]. Chondrocyte cell volume varies with age, being at its maximum in fetuses and early childhood and decreases with age [2]. Hyaluronic acids bind to the collagen macrofibrillar network but also to chondrocytes via the CD44 cell surface receptor. Chondrocytes are also bound to Type II collagen by annexin $\mathrm{V}$ or anchoring $\mathrm{Cll}$ receptors. They synthesize and liberate hyaluronic acid, link protein and aggrecan into the ECM, where they aggregate spontaneously [2], [4].

Other molecules present in articular cartilage include: matrilin-1, fibronectin, tenascin and TSG-6, whose presence is an indicator of 
immaturity or degeneration of new cartilage. The proteoglycan chondroadherin binds the $\alpha_{2} \beta_{1}$ integrin to the chondrocyte surface, promoting adhesion [2]. Other important components are perlecan, which is responsible for matrix organization and matrix ${ }^{-}$ carboxyglutamic acid-rich protein (GLA protein) [2], which prevents matrix calcification. Small proteoglycans such as versican, biglycan, and decorin; thrombospondin and COMP (cartilage oligomeric matrix protein) are also present in articular cartilage [4], [8].

This cartilage is not a homogenous tissue but has different zones in which cellular disposition, collagen fibrils and proteoglycans vary, which in turn can affect its mechanical properties [2].

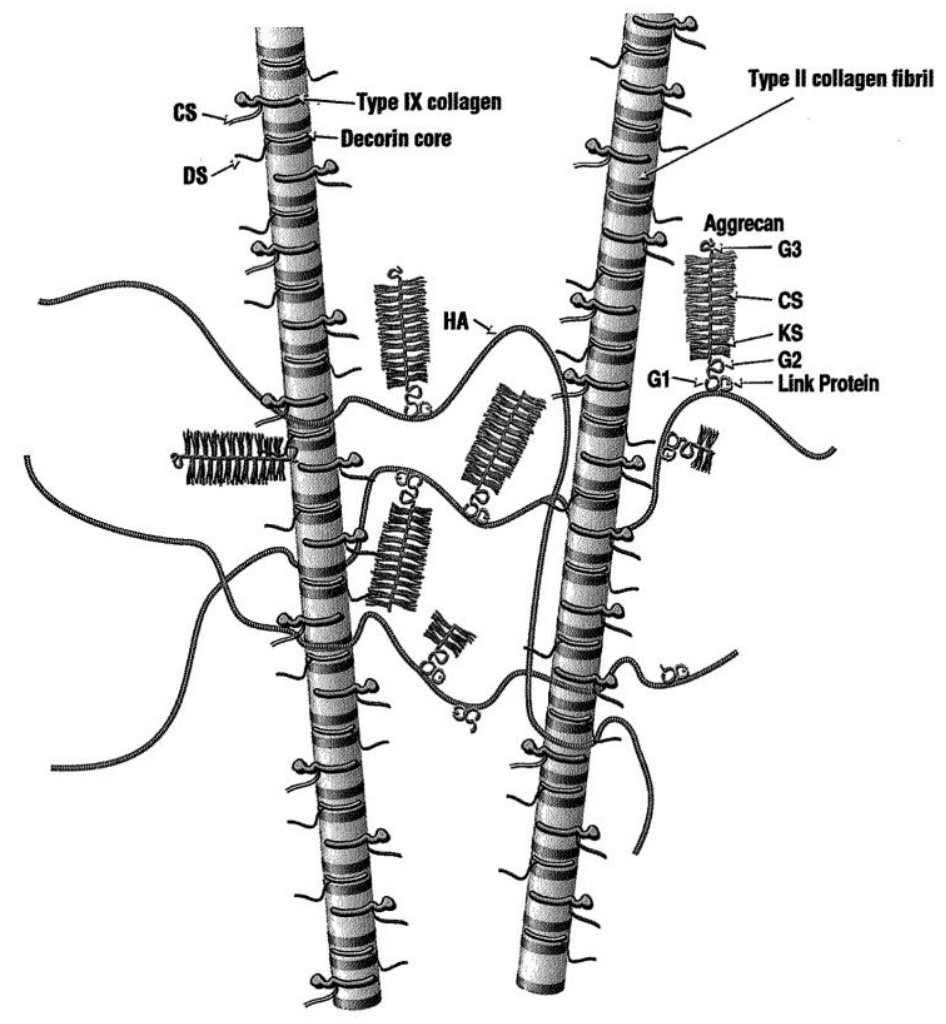

Figure 1-1. Scheme of the macrofibrillar collagen network of articular cartilage and aggrecans linked to hyaluronic acid. CS: chondroitin sulfate; KS: keratan sulfate. Reproduced from [2]. 


\section{Zones of articular cartilage}

Articular cartilage is bounded by a free upper surface that is in contact with the synovial fluid, and a lower surface connected to the subchondral bone. In functional cartilage the different components are arranged differently according to their distance from the free surface. Articular cartilage can be divided into: superficial, middle, deep (or radial) and calcified zones (see Figure 1-2).

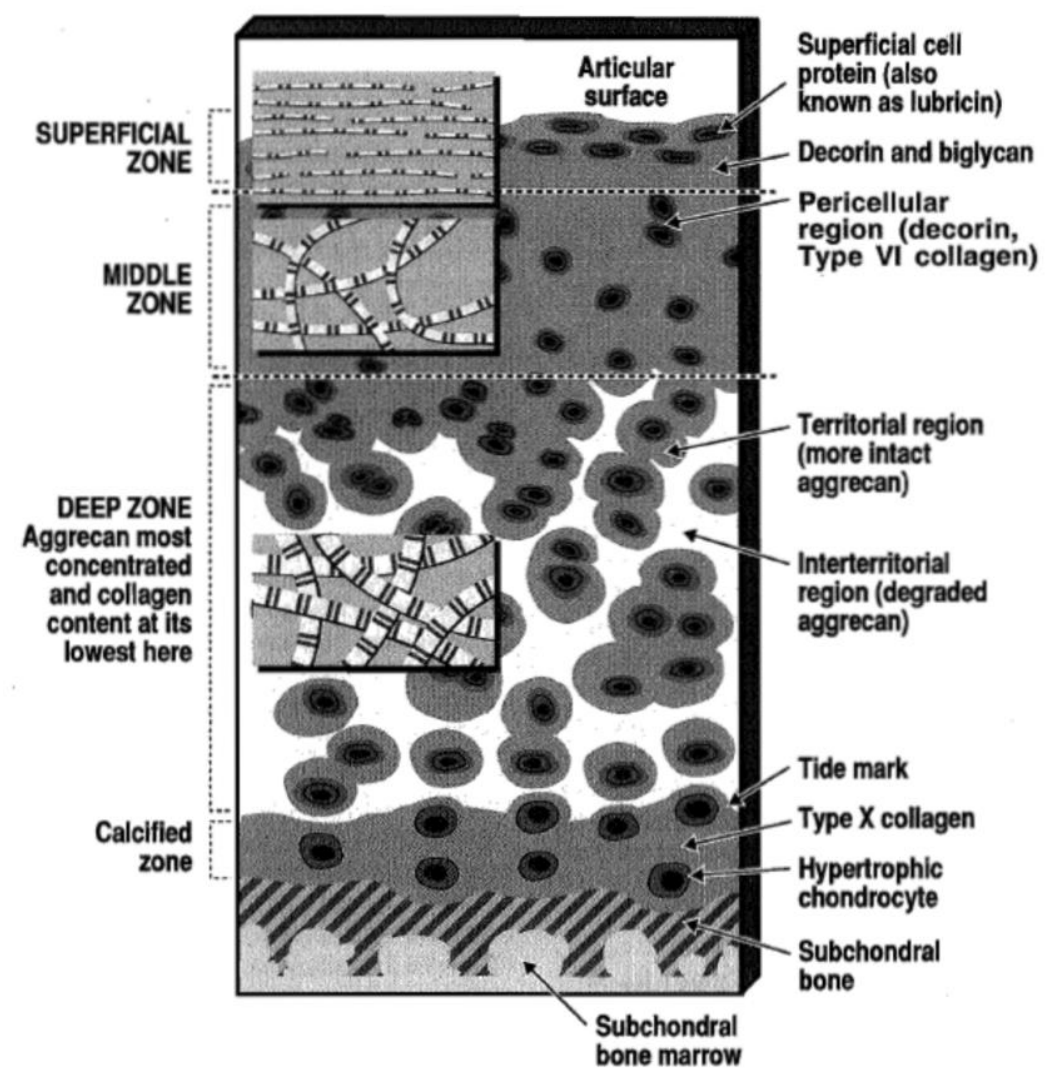

Figure 1-2. Representation of the general structure of articular cartilage showing its main components and the different zones. Image insets show the relative diameters and organization of collagen fibrils. Reproduced from [2]. 


\section{Superficial zone}

This zone occupies $10-20 \%$ of the articular cartilage thickness [9], in which collagen fibrils and chondrocytes are aligned parallel to the articular surface [2], [4]. Chondrocytes have a flattened shape and synthesize lubricin molecules, also known as the superficial zone protein, whose main function is to avoid friction in the joints. The cells are surrounded by a close network of thin collagen fibrils of 20 $\mathrm{nm}$ in diameter that supports matrix organization. Generally, collagen fibers do not offer strong resistance to compression, but are quite strong under tension and also in the superficial zone. The collagen network has the highest tensile properties [2].

The proteoglycan aggrecan is here at its lowest concentration, together with other proteoglycans such as decorin and biglycan [2].

Middle zone

This forms $40-60 \%$ of cartilage thickness [9]. The midzone is below the superficial zone and has the characteristic features of articular cartilage, with rounded cells embedded in a large ECM rich in aggrecan. In this zone collagen fibrils are thicker and less organized. Cell density is lower than in the superficial zone [2].

Deep (or radial) zone

This zone occupies $30-40 \%$ of articular cartilage thickness [9]. Cell density decreases near the bone and is the lowest of all articular cartilage. The collagen fibrils are perpendicular to the subchondral bone surface, they have the largest diameter (from 70 to $120 \mathrm{~nm}$ ) and they are low in content. This zone has the highest aggrecan concentration [2].

\section{Calcified zone}

This is a transition zone between the subchondral bone and the uncalcified cartilage, with intermediate mechanical properties that serve as a link between both types of tissue without compromising mechanical stability. Chondrocytes here present a hypertrophic 
phenotype, synthesizing type $X$ collagen and calcifying the ECM. This calcified ECM resists vascular invasion and is not resorbed during development [2].

\section{Chondrocyte surrounding matrix}

In addition to the organization of articular cartilage in different zones, there is also localized zonation around the chondrocytes. The ECM around the chondrocytes can be divided into three regions (see Figure 1-2):

1) The pericellular region is found in the first $2 \mu \mathrm{m}$ of ECM around the chondrocytes [2], [8]. Few collagen fibrils are found in this region, while Type $\mathrm{VI}$ collagen and the proteoglycans decorin and aggrecan are concentrated here. Type VI collagen forms a branched microfilamentous collagen network of tetramers bound to decorin and associated with hyaluronic acid [2].

In the middle and deep zone the pericellular matrix is separated from the territorial matrix by a fibrous capsule. Together with the pericellular matrix and the chondrocyte they form what is known as "chondron" [8].

2) The territorial region surrounds the pericellular region where the main component is aggrecan [2].

3) The interterritorial region is in the deep zone of cartilage, surrounding the territorial region. It is mainly composed of degraded aggrecan and forms aggregates that have been created as a result of incomplete proteolysis [2].

\subsubsection{Articular cartilage properties}

Articular cartilage has a biphasic nature formed by a fluid and a solid component. As previously stated, proteoglycans are negatively charged in aqueous solution, having a high water retention capacity that is restricted by the collagen network. This composition gives 
articular cartilage one of its main characteristics, its load bearing capacity. When a pressure gradient (hydraulic or osmotic) is applied to articular cartilage, water and electrolytes flow through the permeable porous solid phase and create a load transfer between both phases together with pressurization of the interstitial fluid, giving rise to the compressive and load bearing properties of this cartilage. Its intrinsic characteristics therefore determine some of the tissue's properties, such as permeability, viscoelasticity, compression, tensile and shear behavior and swelling capacity [4]. A summary of articular cartilage's mechanical properties can be seen in Table 1-1.

Permeability is a measure of the capacity of a fluid to flow through a porous and/or permeable material such as articular cartilage. The permeability values obtained for normal human knee cartilage range from $1.14 \times 10^{-15}$ to $2.17 \times 10^{-15} \mathrm{~m}^{4} / \mathrm{Ns}$. When compression stresses are applied pore size is reduced, decreasing permeability. The hydraulic pressure also increases causing higher frictional resistance to fluid flow, which stiffens the articular cartilage by limiting rapid fluid flow rates.

Viscoelasticity is one of the main characteristics of articular cartilage and refers to the behavior of a material when a constant strain or load is applied to it. Creep and stress relaxation are two typical responses of this material (Figure 1-3). In creep, a constant load is applied and the dependence of deformation on time is measured. The initial material deformation rate is quite fast but then slows down until reaching equilibrium, which is reached when the load is balanced and deformation stops. Stress relaxation occurs when a constant deformation is applied to the material. As in creep, the material first responds with high initial stress, which reduces with time until the stress needed for constant deformation is zero and the material is in equilibrium. The main articular cartilage component involved in viscoelastic behavior when a compression force is applied is fluid flow. 
Compression properties. Under normal conditions articular cartilage is mainly subjected to compression loads. The applied load changes the cartilage volume, which increases pressure inside the cartilage and causes fluid to flow through the porous matrix. The interstitial fluid flow then produces frictional resistance with the solid phase of the tissue. The biphasic nature of articular cartilage is responsible for its compressive properties. The tests commonly applied to determine the properties of articular cartilage under compression are the confined compression creep test and indentation tests, in which a constant compression load is applied to the surface of the articular cartilage probe, which is confined in a cylindrical holder with a porous base that permits uniaxial liquid exudation. Initially, the interstitial fluid rapidly exits the cartilage, which deforms until equilibrium is reached. The applied load is first balanced by the solid matrix and the frictional forces exerted by the exuding fluid. As cartilage deformation develops, the stress on the solid matrix increases until it is in balance with the applied load, when fluid flow and matrix deformation cease. If constant displacement is applied instead of a constant load, this is known as a uniaxial confined compression stress-relaxation test. In this case, there is high initial stress which gradually decreases until equilibrium is reached.

Indentation tests have the advantage that they can be applied in situ without the need for discs or tissue probes. In this type of test, the load is applied to the surface of intact cartilage with a cylindrical porous permeable indenter and information is obtained on the rate of fluid exudation and its redistribution within the tissue. 
Table 1-1. Native articular cartilage's mechanical properties and the tests used to measure them. Reproduced from [10].

\begin{tabular}{|c|c|c|c|}
\hline $\begin{array}{l}\text { Mechanical } \\
\text { property }\end{array}$ & Description & Value & $\begin{array}{l}\text { Mechanical } \\
\text { test }\end{array}$ \\
\hline $\begin{array}{l}\text { Aggregate modulus } \\
\text { (MPa) }\end{array}$ & $\begin{array}{l}\text { Equilibrium compressive stiffness } \\
\text { of cartilage constrained at the } \\
\text { sides }\end{array}$ & $0.1-2$ & $\mathrm{CC}, \mathrm{I}$ \\
\hline $\begin{array}{l}\text { Hydraulic } \\
\text { permeability } \\
\left(\mathrm{m}^{4} / \mathrm{Ns}\right)\end{array}$ & $\begin{array}{l}\text { Ease by which interstitial water } \\
\text { moves through the solid ECM }\end{array}$ & $10^{-16}-10^{-15}$ & CC, UC, I \\
\hline $\begin{array}{l}\text { Compressive } \\
\text { Young's modulus } \\
\text { (MPa) }\end{array}$ & $\begin{array}{l}\text { Equilibrium stiffness of cartilage } \\
\text { unconstrained at the sides }\end{array}$ & $0.24-0.85$ & UC \\
\hline Poisson's ratio & $\begin{array}{l}\text { Ratio of lateral strain to strain } \\
\text { along the stress direction and a } \\
\text { measure of the compressibility of } \\
\text { pores in the ECM }\end{array}$ & $0.06-0.3$ & UC,I \\
\hline $\begin{array}{l}\text { Tensile equilibrium } \\
\text { modulus ( } \mathrm{MPa} \text { ) }\end{array}$ & $\begin{array}{l}\text { Tensile stiffness of cartilage at } \\
\text { equilibrium, usually along the } \\
\text { articular surface }\end{array}$ & $5-12$ & TSR \\
\hline $\begin{array}{l}\text { Tensile Young's } \\
\text { modulus (MPa)- } \\
\text { constant- strain } \\
\text { rate }\end{array}$ & $\begin{array}{l}\text { Tensile stiffness of cartilage when } \\
\text { subjected to a constant-strain } \\
\text { rate }\end{array}$ & $5-25$ & TCSR \\
\hline $\begin{array}{l}\text { Tensile strength } \\
\text { (MPa) }\end{array}$ & $\begin{array}{l}\text { Maximum amount of tensile } \\
\text { stress endured by cartilage } \\
\text { before rupturing }\end{array}$ & $0.8-25$ & TCSR \\
\hline $\begin{array}{l}\text { Equilibrium shear } \\
\text { modulus ( } \mathrm{MPa} \text { ) }\end{array}$ & $\begin{array}{l}\text { Measure of the shear stiffness of } \\
\text { solid ECM after all viscous ECM } \\
\text { effects have subsided }\end{array}$ & $0.05-0.4$ & ES \\
\hline $\begin{array}{l}\text { Complex shear } \\
\text { modulus (MPa) }\end{array}$ & $\begin{array}{l}\text { Apparent stiffness of the ECM, } \\
\text { which includes both viscous and } \\
\text { elastic effects }\end{array}$ & $0.2-2.5$ & DS \\
\hline Shear loss angle (ㅇ) & $\begin{array}{l}\text { Measurement of how much of } \\
\text { the complex shear modulus is } \\
\text { caused by viscous effects }\end{array}$ & $10-15$ & DS \\
\hline
\end{tabular}

CC, confined compression; UC, unconfined compression; I, indentation; TSR, tensile stress relaxation; TCSR, tensile constant strain rate; ES, equilibrium shear; DS, dynamic shear. 
a. Creep test
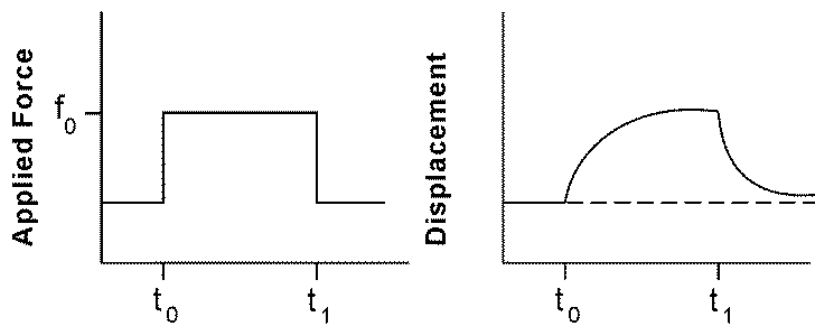

b. Stress-relaxation test
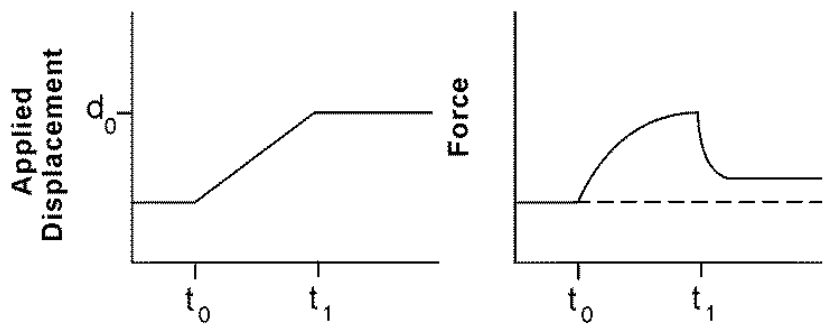

Figure 1-3. Viscoelasticity of articular cartilage. a) Creep test and b) stress-relaxation test. Figure inspired in [11].

Tensile properties. Articular cartilage is normally submitted to compressive forces that also generate tensile stresses within the cartilage matrix because of the collagen network in the ECM, which maintains the structural integrity of the tissue. The viscoelastic behavior of this tissue under tension depends on the movement of the collagen network and the proteoglycans. Under tensile analysis, a small load initially causes a large deformation in the cartilage due to the force required to move the collagen network through the proteoglycans. As the collagen fibers tighten and form a more uniform structure, they become more aligned and can absorb the applied tensile force. When this occurs, the tensile response is linearly elastic at a constant value of the slope in the stress-strain curve, known as the Young's modulus. Since collagen organizes itself differently in the different zones, so does its tensile behavior.

Shear properties. Shear stresses occur when a parallel force is applied to the surface of a material. When this force is applied to articular 
cartilage, they appear in the deep zone near the calcified zone between the layer of articular cartilage and the stiffer subchondral bone due to compression forces. In shear testing, a small shear strain is applied that does not cause any changes in volume or fluid flow. This permits the intrinsic viscoelastic properties of the collagenproteoglycan solid matrix to be calibrated without the effect of the interstitial fluid flow. The studies performed on articular cartilage show that the solid matrix behaves like an elastic solid with only a small viscoelastic component, similar to the collagen response. Although proteoglycans have a lower shear modulus than collagen, they also resist shear forces by keeping the collagen network in place.

Swelling. Articular cartilage has a high swelling capacity. Swelling is caused by the interaction between the highly negatively charged matrix, due to the negatively charged keratan sulfate and chondroitin sulfate, and the ionic constituents of the synovial fluid. The ionic concentration of articular cartilage is higher than the surrounding synovial fluid, and this difference creates osmotic pressure (Donnan osmotic pressure) that forces fluid into the tissue until reaching equilibrium.

The swelling of articular cartilage is also caused by the formation of repulsion forces between the negatively charged sulfate and carboxyl groups of the chondroitin sulfate and keratan sulfate chains (chemical-expansion stress).

When articular cartilage is damaged or degraded its water content increases, which in turn affects its permeability and stiffness.

\subsubsection{Articular cartilage injuries}

Articular cartilage injuries can be classified into two groups: focal lesions and degenerative lesions. The former are the direct result of trauma, osteochondritis dissecans or osteonecrosis, while degenerative lesions are usually caused by meniscal injuries, ligament instability, joint overuse or disuse, obesity, osteoarthritis (OA) or rheumatoid arthritis (RA) [12], [13]. Traumas induced by sporting 
injuries or accidents, together with the most prevalent chondral degenerative process, osteoarthritis, are the most common causes of the degeneration of this type of cartilage. Osteoarthritis usually affects people over 40 and is characterized by the progressive destruction of articular cartilage by uncontrolled proteolysis of its ECM, which also affects the subchondral bone, the synovial membrane and the synovial fluid. Some of the frequent structural changes in degenerated articular cartilage are: fibrillation, chondrocyte proliferation, loss of matrix proteoglycans, subchondral bone thickening, deformation of the articular surface, osteophyte formation and synovial fibrosis [14]. OA is the most common joint disorder in the United States; the prevalence of knee OA is $10 \%$ in men and $13 \%$ in women aged 60 years or older [15].

The main difference between OA and RA is in their origin; OA starts on the cartilage surface while in RA the joint is inflamed and synovial degradative enzymes destroy both cartilage and bone [13].

Articular cartilage can repair itself according to the extent of the damage and this should be taken into account in regenerative therapies. For chondral lesions the Outerbridge classification is the most frequently used in clinical practice (see Table 1-2).

Table 1-2. Outerbridge classification of chondral lesions [12]

\begin{tabular}{cl}
\hline Grade & Description \\
\hline O & Normal articular cartilage \\
I & Softening, blistering or swelling of the cartilage \\
II & Partial thickness fissures and gaps $<1 \mathrm{~cm}$ diameter \\
III & Full thickness fissures to subchondral bone $>1 \mathrm{~cm}$ diameter \\
IV & Exposed subchondral bone \\
\hline
\end{tabular}

Self-repairing processes of natural tissue differ according to the depth of the lesion, e.g. if the lesion affects only a part of the 
cartilage layer (partial thickness lesion) or if it reaches the subchondral bone (full thickness lesion) [16] (see Figure 1-4).

a. Partial thickness defects

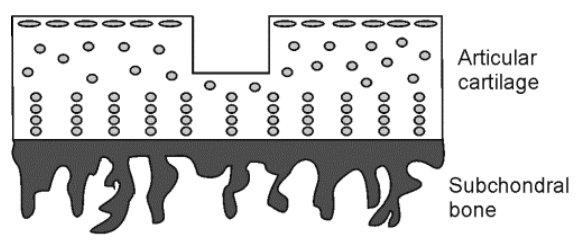

b. Full thickness defects

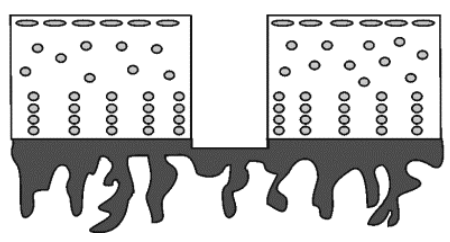

Figure 1-4. Scheme of a) partial thickness defect and b) full thickness defect in articular cartilage. Sketch inspired in [16].

Partial thickness defects, such as small fissures or gaps in the articular cartilage surface, cannot heal spontaneously. The reason is thought to be the absence of progenitor cells from the bone marrow space, since articular cartilage has no blood vessels. After receiving an injury there is thus no angiogenesis response and undifferentiated cells do not invade the injured tissue (see Figure 1-4a). However, the cells close to the injury site do respond immediately. Firstly, the cells adjacent to the wound margins undergo cell death followed by an increase of chondrocyte proliferation or cell cluster formation after 24 hours. This cell proliferation increases the synthesis of ECM and catabolism processes. This response is very short and does not succeed in repairing the defect. It has also been observed that cells can migrate through the synovial fluid to the injury site, but the antiadhesive properties of the proteoglycans that form articular cartilage do not allow them to settle there. However, the full repair mechanism and the factors that influence articular cartilage repair are still unknown.

In full thickness defects, the injury reaches the subchondral bone and causes the migration of mesenchymal stem cells from the bone 
marrow (see Figure 1-4b). These cells fill the defect with fibrocartilage tissue. As this new tissue does not have the same mechanical properties as native tissue, this can lead to the failure of the repaired tissue and continued cartilage degeneration.

\subsubsection{Articular cartilage repair therapies}

The therapies currently applied to treating articular cartilage injuries or degeneration are aimed at reducing pain or at restoring damaged tissue. To date, only fibrocartilaginous tissue has been synthesized. This tissue has lower mechanical properties than native hyaline cartilage and with time leads to new tissue failure and further degradation of the cartilage. The selection of the treatment will depend on patient characteristics such as age, sporting activity and grade and type of lesion and can be classified into conservative treatments and surgical procedures.

\section{Conservative treatments}

These are aimed at palliating the symptoms without surgical intervention, not being reparative treatments. They are used in small lesions or low grade injuries where surgical treatment would cause more damage. Some of the treatments usually used are medication, mechanical treatment (weight loss, ice, canes, rest, physical therapy, etc.), nutritional supplements (chondroprotective agents, calcium and vitamins) or intraarticular injections (steroids or viscosupplementation) [12].

\section{Surgical treatments}

The main purpose of a surgical procedure is to regenerate osteochondral defects by tissue with similar characteristics, such as hyaline cartilage. However, present-day treatments can only lead to the formation of fibrocartilaginous tissue, which will fail after a certain period. In some cases, such as obesity, infection, inflammatory arthropathy or unstable joints, surgical treatment is not a good option. 
- Arthroscopy lavage and debridement is not a regenerative technique. Its main purpose is to diminish pain in cases of minor injuries. In this type of treatment inflammatory mediators and loose cartilage debris are washed out of the synovium fluid in order to prevent synovitis. Cartilage debridement, or chondroplasty, involves removing cartilage or meniscal fragments [12]. In abrasion arthroplasty, the edges of the articular cartilage defect are debrided to obtain a uniform contour of non-degraded collagen, to which a fibrin clot can be adhered. The subchondral bone is then breached to allow blood to reach the defect and form a fibrin clot [12].

- In subchondral drilling, as in the previous treatment, the boundaries of the defect are debrided but in this case, in order to obtain blood perfusion, a high speed drill is used to penetrate the subchondral bone and reach the trabecular bone. Blood can then reach the defect to form the blood clot and initiate the repair. The main risk of this technique is thermal necrosis. The synthesized tissue is formed by a mix of fibrous and hyaline cartilage [12].

- In microfracture, an arthroscopic awl is used to create several holes on the articular cartilage surface to increase roughness and improve fibrin clot formation [12].

- Mosaicoplasty - Osteochondral autografting (OATS) aims at regenerating osteochondral defects with autografts from a nonweight bearing area as donor when no immune response is expected. Lesions derived from degenerative processes cannot be treated in this way, only local defects produced by a trauma. Some of the steps in the procedure are similar to those previously described. First, arthroscopic debridement is performed, followed by removing unstable cartilage to form a circular shape. The circular defect is then extended into the subchondral bone. A cylindrical osteochondral plug of the same dimensions as the treated defect is harvested from the donor area and inserted into the defect [12].

- Osteochondral allografting uses the tissue from a donor of the same species. This type of transplant is often used in medium to large full- 
thickness lesions when the graft size is limited and other techniques cannot be applied. It also avoids donor site morbidity. [12].

- Autologous chondrocyte implantation (ACI) was first used in 1994. It is usually recommended for young patients (20-50 year olds) with a femoral lesion bigger than 2-4 $\mathrm{cm}^{2}$ when initial repair of the subchondral bone is not necessary. In the first generation of $\mathrm{ACl}$, autologous chondrocytes are harvested and proliferated in the Good Manufacturing Practices (GMP) laboratory for six weeks. Chondrocytes are injected into the defect after debridement, the cartilage defect is measured, the periosteal flap is harvested from the medial tibia in the shape of the defect and the flap is fixed in place. Some difficulties involved in this technique are: multiple staged procedures, long operation times, difficult access to certain areas for suturing, long rehabilitation time and periosteal flap complications. To overcome periosteal flap problems a collagen type I/III membrane is used in what is called the second $\mathrm{ACl}$ generation. Using a collagen membrane instead of a periosteal flap avoids graft hypertrophy [12].

- The aim of matrix-induced $\mathrm{ACl}(\mathrm{MACl})$ is to minimize the surgical procedures and periosteal complications associated with the $\mathrm{ACl}$ technique. Its main difference to $\mathrm{ACl}$ is that it uses a collagen scaffold instead of the periosteal flap or collagen membrane and the loose injection of chondrocytes [16]. Cultured autologous chondrocytes are seeded onto a bilayer collagen $\mathrm{I} / \mathrm{III}$ membrane that is implanted in the cartilage defect and attached with fibrin glue. One side of the collagen bilayer is seeded with the cells and its porosity allows matrix synthesis and tissue integration. The other side is a smooth hyalinelike surface that reduces the friction with the chondral surface and acts as a barrier against soft tissue invasion.

The steps of the surgical intervention are similar to the $\mathrm{ACl}$ technique. An arthroscopic diagnosis of the lesion is followed by chondral biopsy to determine the chondrocytes to be cultured in the GMP laboratory. The chondrocytes are then seeded on the collagen membrane and the defect is debrided, shaped and sized and the 
membrane is implanted with fibrin glue. The main advantages of this technique are: no periosteal harvesting, less invasive, suture free, good stability of the implant and early mobilization [12], [16].

- Artificial chondroplasty implants (e.g. Co-Cr metallic implants) are often used with elderly patients when no other options are available. Most of these are experimental and are used in focal lesions [12].

- Other techniques under study. Other studies for the regeneration of articular cartilage are currently being carried out, since the current therapies are still not able to properly regenerate damaged tissue. Autologous chondrocytes in the $\mathrm{ACl}$ and $\mathrm{MACl}$ techniques have not been successful in the development of appropriate cartilage tissue, since they dedifferentiate to a fibroblast-like phenotype when monolayer cultured in the laboratory [17]. This underlines the need for further research into chondrocytes and other cell types for articular cartilage regeneration. Also, the collagen scaffold used in $\mathrm{MACl}$ does not have the adequate mechanical properties to correctly stimulate chondrocytes, does not interpenetrate with the surrounding host tissue and does not provide the biological cues and properties of articular cartilage needed for the cells to synthesize the ECM components, which finally leads to failure of the scaffold. There is also a need to characterize cell behavior in new scaffolds that better mimic articular cartilage properties. In fact, the main aim of this thesis is to obtain a scaffold that better mimics articular cartilage properties in order to improve cell survival and ECM production.

Current research strategies can be classified into three groups: the use of other cell lines, of other types of hydrogels or scaffolds, and of growth factors and gene therapy [12].

When other cell lines are replaced by chondrocytes, most studies employ mesenchymal stem cell (MSCs) implants seeded in hydrogels or scaffolds. MSCs have several advantages over chondrocytes: they can be obtained from a wide variety of tissues, e.g. bone marrow, adipose tissue, synovium, periosteum, umbilical cord vein or placenta. They also have a high expansion capacity in vitro and can be 
differentiated into chondrocytes under the appropriate conditions [18]. Fibroblasts are also being studied, since they can be directed towards a chondrocytic phenotype when cultured in the right conditions [19].

However, the cells cannot be directly introduced into the defect, since this would involve cell diffusion outside the injection site to other parts of the organism. Like $\mathrm{MACl}$, the use of a matrix or scaffold that can maintain cells in the defect site and support the mechanical loads in the first stages of implantation until the cells start to synthesize their own ECM seems to be a good repair strategy. However, the collagen scaffold used in the $\mathrm{MACl}$ technique does not provide the appropriate mechanical and biological signals for the formation of articular cartilage. $\mathrm{MACl}$ technique treatments have been shown to cause tissue hypertrophy, incomplete filling and limited integration with surrounding normal cartilage [20]. This shows that scaffolds with properties similar to native articular cartilage (high swelling, similar chemistry and compression properties, etc.) and that can integrate with the surrounding cartilage need to be developed.

Regarding the use of growth and differentiation factors, TGF- $\beta 1$ and TGF- $\beta 2$ have shown an increase in aggrecan and collagen gene expression and have prevented proteoglycan loss. However, they are not the perfect option, as some therapeutic studies with TGF- $\beta 1$ resulted in joint fibrosis and osteophyte formation. Bone morphogenetic proteins (BMPs) have also been proposed in combination with MSCs, as they are able to induce chondrogenic differentiation, at least in in vitro cell cultures. However, more trials need to be done to assess whether their behavior is as good as the TGF- $\beta$ factors. Other growth factors currently under study are: the Fibroblast Growth Factor (FGF), Insulin Growth Factors (IGFs), the Wingless (Wnt) family and Hedgehog family [14], [18]. Other approaches aim to mediate in $\mathrm{OA}$ by inhibiting inflammatory mediators (IL-1 and TNF- $\alpha$ ) or other substances that play a role in cartilage degeneration (MMPs, ADAMTS-4 and ADAMTS-5) [14]. The 
gene therapy approach is focused on manipulating progenitor cells and chondrocytes to locally express genes that encode specific growth factors, which will enhance osteochondral repair [12], [18].

\subsection{Hydrogels}

Hydrogels are among the most important materials studied in cartilage tissue engineering. They are composed of hydrophilic polymeric chains linked to each other through a network of crosslinking points. The hydrophilic chains give them the ability to absorb great quantities of water and at the same time the crosslinking points prevent their dissolving in water or physiological buffers. They are able to imitate the extracellular matrix of articular cartilage, which is highly hydrated due to the presence of proteoglycans. Hydrogels have many medical applications, not only in cartilage regeneration but also as controlled protein or drug delivery carriers [21]-[25], as supports for the proliferation and differentiation of cells [26]-[34] and as barriers between tissue and material surfaces [35]-[39].

There are different types of hydrogel crosslinking: (a) by covalent bond between the monomeric units of the polymeric chains and the crosslinker molecules, (b) by physical chain entanglement, (c) by secondary bonds (hydrogen bonds and van der Waals interactions) between the polymeric chains, or (d) by crystallites able to join together two or more polymeric chains [40], [41]. When the hydrogel is covalently bonded it is said to be a chemical hydrogel and a physical hydrogel when held together by molecular entanglements and/or secondary forces.

Different polymers can be combined to obtain a hydrogel with the desired characteristics of the tissue to be regenerated (swelling, mechanical, permeability, etc.). Examples of these are: homopolymers, blends of two or more polymers, copolymers (random, graft, alternating or block copolymer [42]) or 
interpenetrated polymer networks (IPNs) [41] (see Figure 1-5). The final structure and properties of the hydrogel obtained will depend on the monomers used as well as the synthesis process. Both of these are determinants in the monomer sequence of the polymeric chains and their interactions, crosslinking density, equilibrium water content, degradation profile and final mechanical properties [43][45].

- Polymer A - Crosslinker A

- Polymer B - Crosslinker B

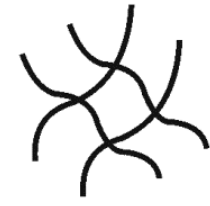

Homopolymer

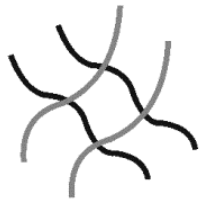

Polymer Blend

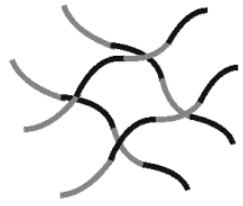

Copolymer

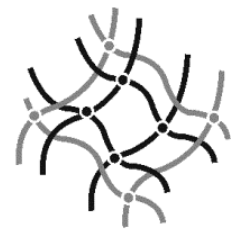

IPNs

Figure 1-5. Types of polymeric structures.

Hydrogels can be classified into natural or synthetic hydrogels.

Synthetic hydrogels have better and more consistent mechanical properties than natural hydrogels and can be obtained on a larger scale. They are highly biocompatible but most need modifications by degradable groups to increase their biodegradability (e.g. polyesters, MMP-sensitive peptides, photodegradable groups, etc.) [43].

Poly(hydroxyethyl methacrylate) (PHEMA), discovered in 1960 [46], was the first material used in soft contact lenses [41]. It is weak, biocompatible and inert and its hydrophilic nature hinders protein and cell adhesion, as in most synthetic hydrogels. It has many other biomedical applications, as for instance artificial skin [47] and drug delivery [48], [49].

Poly(vinyl alcohol) and poly(ethylene glycol) are used in medicine. The former has compression, tensile and shear moduli, as well as permeability, similar to articular cartilage. As it has a high water sorption capacity and good biocompatibility its use in tissue 
engineering is conditioned by the incorporation of biological factors, as its protein adsorption and cell adhesion are quite poor.

Poly(ethylene glycol) (PEG) has very good mechanical properties [50], immunogenicity and biocompatibility, but limited protein binding and cell adhesion [51]. It is being explored as a substrate for the encapsulation of cells in cartilage tissue engineering, with promising results in cell viability and synthesis of cartilaginous ECM [30], [44], [52]-[54].

Natural hydrogels are inherently biocompatible and more environmentally friendly than those produced synthetically. However, they are difficult to produce on a large scale, tend to be softer than synthetic hydrogels and it is difficult to control their properties. They are degraded by specific enzymes and the degradation kinetics is less reproducible and predictable than hydrolysis.

Most natural hydrogels used as biomaterials can be classified as either polysaccharides or proteins. The principal polysaccharides are: chondroitin sulfate, hyaluronic acid, alginate, agarose, chitosan and cellulose. The proteinic types include fibrin, collagen, gelatin, elastin and fibroin [40].

Since articular cartilage ECM is a combination of the protein collagen II and polysaccharides (mainly in the form of GAGs), recent research has focused on scaffolds (or hydrogels) combining proteins and polysaccharides as potential biomimetic extracellular matrices.

\section{Protein-polysaccharide hydrogels}

Many research groups have focused their studies on the use of scaffolds of collagen type I and type II for MSC differentiation towards the cartilaginous phenotype [55]-[58]. Du et al. studied the differentiation of human MSCs (hMSCs) into chondrocytes cultured in a chondroitin sulfate-collagen hydrogel synthesized through a $\mathrm{pH}-$ triggered $(\mathrm{pH}$ 2-3) co-precipitation [59]. hMSCs differentiation was controlled by the release of growth factors (TGF- $\beta$ and bFGF) bonded 
to the CS-collagen hydrogel. Collagen has also been combined with chitosan [60], [61] hyaluronic acid [62]-[64] and cellulose [65]. Biomimetic hydrogels of articular cartilage include not only collagen and one polysaccharide, but two typical cartilage polysaccharides, CS and HA [66].

Gelatin derived from collagen hydrolysis has been proposed as cheaper and easier to obtain than collagen. It has been combined with CS [67], [68], hyaluronic acid [69], chitosan [70], [71], cellulose [72], agarose [73] and alginate [74] polysaccharides. Tri-copolymers of gelatin with CS and HA [75]-[78], with chitosan and HA [79], and with chitosan and agarose [80] have also been studied.

Fibrin has also been extensively used as mimetic hydrogel of the ECM for cartilage regeneration. This protein plays an important role in blood coagulation, inflammation processes, wound healing and cellmatrix interactions as it is a protein with a fast degradation rate in the organism. Park et al. obtained fibrin-HA hydrogels with fibrinolysis inhibition factors as cell delivery vehicles of chondrocytes and tested them in a rabbit model [81]. Pereira et al. synthesized injectable carrageenan/fibrin/hyaluronic acid-based hydrogels that showed good regeneration in sheep cartilage lesions with the help of human articular chondrocytes [82]. Gamboa-Martínez et al. tested chitosan microspheres within a fibrin matrix. The fibrin/chitosan combination supplied suitable domains for chondrocyte anchoring. Human chondrocytes actively produced collagen type II and GAGs after 28 days of culture [83].

Silk fibroin has a structure that consists of glycine, alanine and serine [40]. It is a natural fibrous protein with good permeability to oxygen and water, relatively low thrombogenicity, low inflammatory response, good cell adhesion and proliferation and high tensile strength. However, its main disadvantage is its brittleness [84]. Bhardwaj et al. cultured silk fibroin-chitosan scaffold with bovine chondrocytes for two weeks. They found that the presence of chitosan in the silk fibroin scaffold gave enhanced GAG and collagen 
synthesis [85]. Many studies have combined these two natural polymers to obtain a composite scaffold with better physical and biological properties than pure polymers. Its non-cytotoxicity and degradable properties have also been studied [84], [86]. Fibroin aggregation is restrained by the addition of HA. Garcia-Fuentes et al. synthesized silk fibroin-HA hydrogel blends cultured with hMSCs that gave better collagen I and III expression than pure silk fibroin scaffold [87]. Ren et al. also prepared fibroin-HA hydrogels and showed that they are cytocompatible and suitable for neural cell survival, migration and adhesion [88].

\subsection{Gelatin-Hyaluronic acid hydrogels}

\subsubsection{Gelatin}

Gelatin is a natural polymer derived by partial denaturation of collagen, the main structural protein of the extracellular matrix of most tissues. It is not formed by a single chemical entity but a mixture of fractions composed of amino acids joined by peptide linkages to form polymers varying in molecular mass from 10000 to $400000 \mathrm{~g} / \mathrm{mol}$ [89]. It is enzymatically degraded by collagenases, which degrade the gelatin chain cleaving the peptide bonds [5], [90], [91].

Both gelatin and collagen are made up of 20 amino acids. The most common of these are glycine, proline and hydroxyproline (see Figure 1-6). The composition of collagen and gelatin amino acid is given in Table 1-3. The main functional groups of gelatin are amine, carboxyl and hydroxyl groups, which can be bonded with other active molecules to modify its properties (mechanically or chemically via crosslinking). 


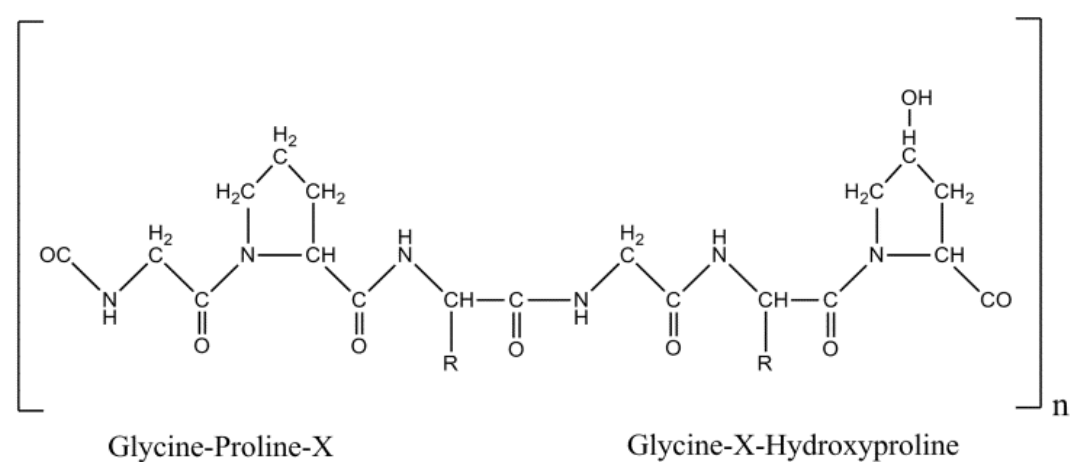

Figure 1-6. Representative structure of gelatin [91].

Table 1-3. Amino acid composition of acid-conditioned gelatin (Type A), alkali-conditioned gelatin (type B) and collagen. Residues per 1000 residues [89].

\begin{tabular}{|c|c|c|c|}
\hline Amino Acid & Gelatin Type A & Gelatin Type B & $\begin{array}{c}\text { Type I Collagen } \\
\text { (cattle) }\end{array}$ \\
\hline Alanine & 112 & 117 & 114 \\
\hline Arginine & 49 & 48 & 51 \\
\hline Asparagine & 16 & 0 & 16 \\
\hline Aspartic Acid & 29 & 46 & 29 \\
\hline Cysteine & - & - & - \\
\hline Glutamic Acid & 48 & 72 & 48 \\
\hline Glutamine & 25 & 0 & 25 \\
\hline Glycine & 330 & 335 & 332 \\
\hline Histidine & 4 & 4.2 & 4.4 \\
\hline Hydroxiproline & 91 & 93 & 104 \\
\hline Hydroxilisine & 6.4 & 4.3 & 11 \\
\hline Isoleucine & 10 & 11 & 24 \\
\hline Leucine & 24 & 24.3 & 28 \\
\hline Lysine & 27 & 28 & 5.7 \\
\hline Methionine & 3.6 & 3.9 & 13 \\
\hline Phenylalanine & 14 & 14 & 115 \\
\hline Proline & 132 & 124 & 35 \\
\hline Serine & 35 & 33 & 17 \\
\hline Threonine & 18 & 18 & -4 \\
\hline Tryptophan & - & - & 22 \\
\hline Tyrosine & 2.6 & & \\
\hline Valine & 26 & 1.2 & 22 \\
\hline
\end{tabular}


Gelatin has attracted attention as a hydrogel scaffold for tissue engineering due to its biocompatibility, biodegradability [92]-[94] and the presence of different integrin binding sites for cell adhesion and differentiation [95]. It forms a physical hydrogel below its melting temperature $\left(\approx 23^{\circ} \mathrm{C}\right)$ by the organization of some chains into helical structures stabilized by intramolecular bonds acting as crosslinking points [96]. Above gelation temperature these helical structures are not stable and gelatin becomes soluble in water. Gelatin-based scaffolds are thus usually crosslinked by chemical agents covalently linking different chains. Examples include: glutaraldehyde, genipin, carbodiimides and diisocyanates [40], [78], [97]-[101]. However, crosslinking reactions with these agents are usually cytotoxic and cannot occur in the presence of cells.

\subsubsection{Hyaluronic acid}

Hyaluronic acid $(\mathrm{HA})$, also known as hyaluronan, is a negatively charged glycosaminoglycan composed of D-glucuronic acid and D-Nacetylglucosamine linked via $\beta-1,4$ and $\beta-1,3$ glycosidic bonds (see Figure 1-7). It is degraded enzymatically by hyaluronidases which cleave the $\beta-1-4$ glycosidic linkages in HA producing tetraoligosaccharides and hexa-oligosaccharides as the major end products [102].

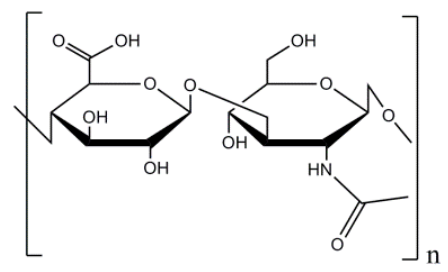

Figure 1-7. Chemical structure of hyaluronic acid.

HA is one of the major components of cartilage ECM and other soft tissues. Healon ${ }^{\circledR}$ (Abbot Medical Optics) was the first medical product based on HA developed in 1970 for use in eye surgery. It is still being used as a surgical aid in cataract extraction, intraocular lens 
implantation, corneal transplant, glaucoma filtration and retinal attachment surgery. Many other HA-based products have been developed for different uses: injection of intraarticular HA to treat $O A$, tissue augmentation (e.g. Hylaform ${ }^{\circledR}$, etc.), wound dressing, blood barrier and tissue engineering [103]. Currently, HYAFF ${ }^{\circledR}$ is the most frequently used hyaluronan-based commercial product. HYAFF$11^{\circledR}$ is an esterified form of hyaluronan that produces a highly hydrophobic polymer with a degradation time of 40 days [104].

As this polysaccharide is soluble in water, chemical crosslinking has been proposed to form physiologically stable hydrogels. The most common groups of HA used for crosslinking are the hydroxyls and carboxyls. Hydroxyl groups form ether bonds while carboxyl groups form ester bonds. The HA backbone can be modified to obtain other reactive groups or to include the RGD cell adhesion sequence. Some of the HA crosslinking strategies use carbodiimides (e.g. 1-ethyl-3-(3dimethyl aminopropyl) carbodiimide/ $\mathrm{N}$-hydroxysuccinimide, EDC/NHS), hydrazides, aldehydes, divinyl sulfone, photocrosslinking, autocrosslinking and enzymes [53], [105]-[107]. More recent strategies are able to produce injectable HA hydrogels in which the HA solution is charged with cells, injected in the site of the defect and in situ crosslinked [40].

It has also been proposed as a good candidate for cartilage tissue engineering, jointly with some HA derivatives (methacrylated HA, tyramine modified, etc.) [26], [106], [108]-[112]. Mixtures with other components such as fibrin [81], [113], collagen [64], [114], gelatinchondroitin sulfate [75], [115] and chitosan [116] have also been studied as potential materials for tissue engineering.

\subsubsection{Extracelular-matrix inspired hydrogels of gelatin and hyaluronic acid}

One of the strategies followed in the present work to obtain a suitable scaffold for cartilage regeneration is the combination of gelatin and hyaluronic acid. This combination mimics articular 
cartilage composition and produces a hydrogel with the cell adhesion properties of gelatin and the higher swelling and mechanical properties of HA hydrogels. This strategy has already been proposed by other authors and a summary of their conclusions are described here.

Shu et al. synthesized a disulfide crosslinked thiolated gelatinhyaluronic acid hydrogel. Enzymatic degradation studies with collagenase showed that the incorporation of hyaluronic acid into the gelatin hydrogel retarded its degradation. On the other hand, the presence of gelatin in the HA hydrogel improved attachment of Balb/c murine 3T3 fibroblasts on the hydrogel surface [117]. Weng et al. obtained hydrogels oxidizing hyaluronic acid in order to generate aldehyde groups, which were then crosslinked with gelatin. In vitro tests with fibroblasts showed that cells attach to and infiltrate into the hydrogels. Degradation assay of hydrogels in the presence of cells and in culture medium indicated that hydrogel degradation rates were controlled by the oxidation degree of oxidized HA, which correlates with the crosslinking density. Hydrogels with $50 / 50 \% \mathrm{w} / \mathrm{w}$ ratio but changing the $\mathrm{HA}$ oxidation degree, showed that disintegration time increased from 11 to 32 days when the oxidation degree was raised from 16.7 to $23.4 \%$. Beyond this ratio, hydrogels with lower gelatin content degraded faster (in 7 days), mainly due to the stabilization effects of gelatin's physical crosslinking. In vivo studies also indicated that these hydrogels have excellent biocompatibility and are biodegradable [118]. Chen et al. also obtained oxidized HA-gelatin hydrogels as materials for the regeneration of the nucleus pulpous. The encapsulated nucleus pulpous cells survived and proliferated. Importantly, cells synthesized COL2A1, AGN, SOX-9, BGN, DCN, and HIF-1A mRNA [119].

Choi et al. prepared gelatin-hyaluronic acid sponges crosslinked with EDC. Biodegradation was studied with $13 \mathrm{U} / \mathrm{mL}$ collagenase solution in phosphate-buffered saline (PBS). Collagenase cleaves amide bonds within the triple helical structure of collagen and has specificity for 
the Pro-X-Gly-Pro-Y region, splitting $X$ and Gly. The results obtained indicated that hydrogels with higher crosslinking have greater resistance to collagenase degradation. In vivo tests in rats confirmed that sponges impregnated with an antibiotic used to treat burns can aid in healing skin defects [91]. Zhou et al. also prepared gelatin-HA blends by freeze-drying crosslinked with EDC. In this study the authors indicate that the equilibrium swelling ratio increased with higher HA content, but decreased with higher crosslinker content [120].

Liu et al. synthesized a collagen-gelatin-hyaluronic acid film to be used in cornea tissue engineering. They obtained a film with hydrophilicity, optical performance, mechanical and diffusion properties similar to the human cornea. The viability study with human corneal epithelial cells demonstrated good cellular compatibility [94].

Vanderhooft et al. synthesized hydrogels of thiol-modified carboxymethyl HA and thiol modified gelatin, crosslinked using poly(ethylene glycol)-bis-acrylate. The storage shear moduli of HA hydrogels ranged from $11 \mathrm{~Pa}$ to $3.5 \mathrm{kPa}$, being within the range of soft tissues [121].

Camci-Unal et al. synthesized methacrylated gelatin and $\mathrm{HA}$ hydrogels by photopolymerization. The presence of gelatin induced cell spreading of human umbilical cord vein endothelial cells (HUVECS) while the presence of HA improved hydrogel mechanical properties [122]. Levett et al. also prepared photopolymerized methacrylated gelatin-HA. Their results showed that encapsulated chondrocytes kept their rounded shape and that secreted extracellular matrix increased the compressive modulus of the hydrogels by up to three-fold over 8 weeks of culture. Importantly, cells substantially increased the stiffness of the synthesized hydrogels [123].

Most chemical crosslinking reactions are cytotoxic and leave behind solvents, initiators or unreacted substances that often produce 
inflammation and cell death [124]. These hydrogels are thus preformed in the lab and thoroughly washed to remove undesired reactants prior to their clinical application. Photocrosslinked hydrogels have additional drawbacks, since monomer solution limits light penetration and some of them change their transparency as the reaction progresses, diminishing hydrogel homogeneity [125].

To overcome these problems, there has recently been increasing interest in the development of injectable hydrogels capable of crosslinking in suitable conditions with the use of enzymes, which offers considerable advantages over preformed hydrogels [107], [126], [127]. In the following section we summarize some leading studies that use enzymatic crosslinking, with special attention to gelatin and hyaluronic acid.

\subsection{Injectable enzymatically crosslinked hydrogels of gelatin and hyaluronic acid}

Non-cytotoxic enzyme mediated crosslinking reactions have recently been proposed as an alternative to achieve the added advantage of enabling injectability of the gel precursor with the embedded cells on the site of the tissue defect, since they gel/crosslink within a few minutes [128]-[130]. The desirable characteristics of an injectable hydrogel are:

(i) No cytotoxic crosslinking.

(ii) Fast and controlled crosslinking to keep cells within the injection site, adapting their shape to the defect and avoiding mechanical failure.

(iii) Easy, effective and homogeneous encapsulation of cells and therapeutic molecules.

(iv) Provides cell adhesive sequences to maintain cell viability, promote cell adhesion and differentiation. 
(v) Possesses sufficient mechanical stability to be able to act as a temporary scaffold while the cells produce their own ECM and form new tissue.

(vi) Minimally invasive in vivo implantation and fast patient recovery, with small scar size and reduced pain.

(vii) Good interaction with the host tissue by crosslinking with native extracellular matrix.

Several years ago, tyramine conjugates of the polysaccharides hyaluronic acid [131] and dextran [132] were presented as injectable hydrogels for tissue engineering due to their ability to crosslink by enzyme-mediated non-cytotoxic reactions. Later, Sakai et al. demonstrated that similar derivatives of gelatin exhibited $95 \%$ viability of encapsulated cells with successful in vivo gelification after subcutaneously injecting the precursor solution into rodents [129].

Gelatin and hyaluronic acid chains need to be modified in order to allow enzymatic crosslinking. In the present work tyramine was grafted onto both gelatin and hyaluronic acid chains using EDC and NHS. Firstly, the gelatin or hyaluronic acid chains react with the EDC forming an O-acylisourea [133] (Figure 1-8).

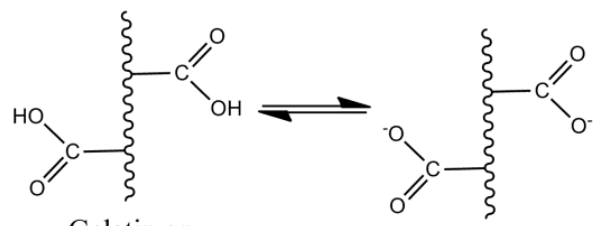

Gelatin or Hyaluronic Acid<smiles>[R]N=C=N[Z]=C[IH+]</smiles><smiles>[R]N=C(N[R])OC(=O)C(C)C(=O)OC(=O)C(=O)OCC</smiles>
2 EDC

Figure 1-8. Formation of EDC carbocation (1) and of O-acylisourea (2). 
The O-acylisourea then reacts with NHS (which acts as a stabilizer) and the amine group of tyramine in order to form an amide linkage and obtain the tyramine grafted gelatin (Gel-Tyr) or tyramine grafted hyaluronic acid (HA-Tyr) (Figure 1-9).
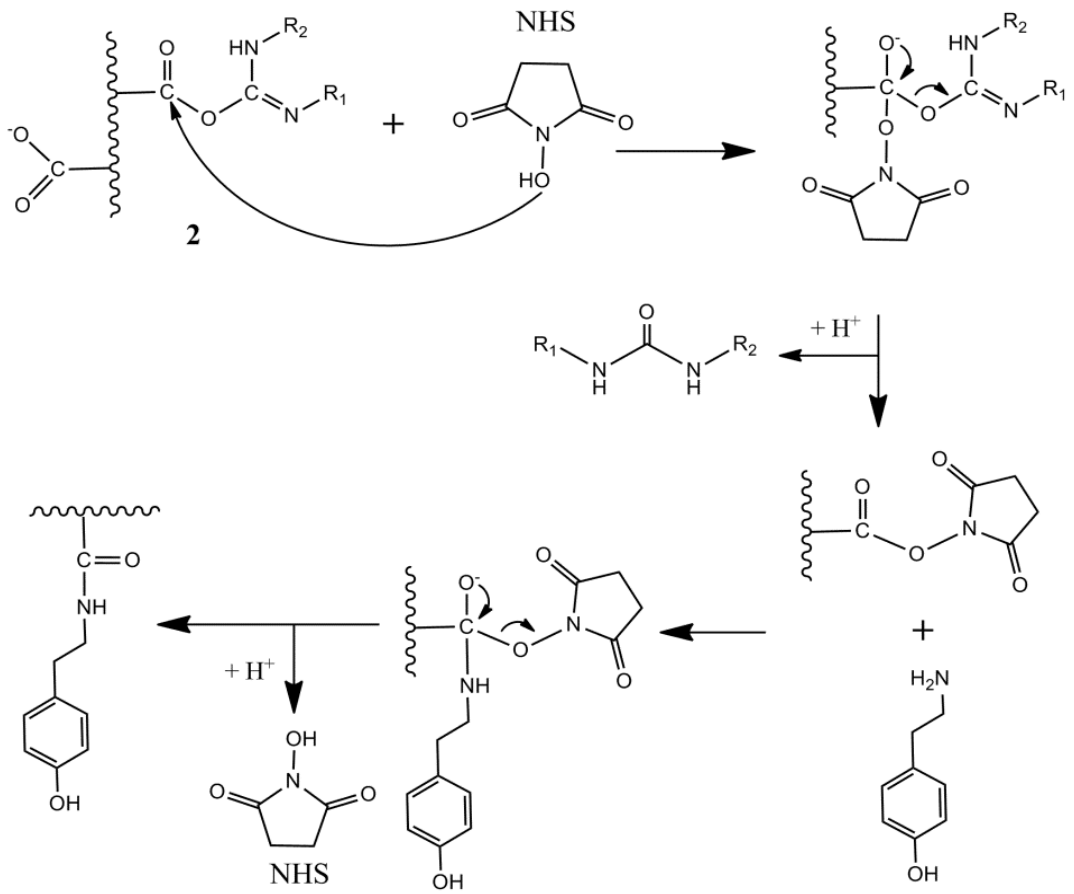

Tyramine

Figure 1-9. Reaction of O-acylisourea with NHS and tyramine to form an amide.

Once the tyramine is grafted onto the polymeric chains, they can be crosslinked with horseradish peroxidase (HRP) and hydrogen peroxide $\left(\mathrm{H}_{2} \mathrm{O}_{2}\right)$, see Figure 1-10. $\mathrm{H}_{2} \mathrm{O}_{2}$ acts as the oxidant of the tyramine molecule, and the HRP catalyzes the crosslinking reaction, oxidizing two tyramine molecules every crosslinking cycle [134]. Soybean peroxidase or hematin can be used as the catalysts instead of HRP [128], [135]. 


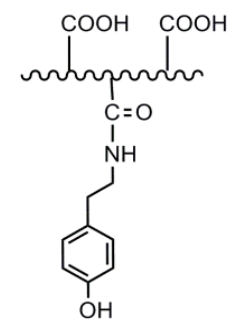

Gel-Tyr or HA-Tyr

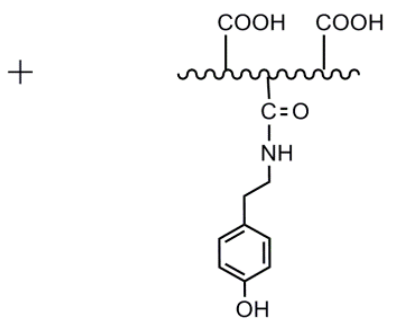

Gel-Tyr or HA-Tyr

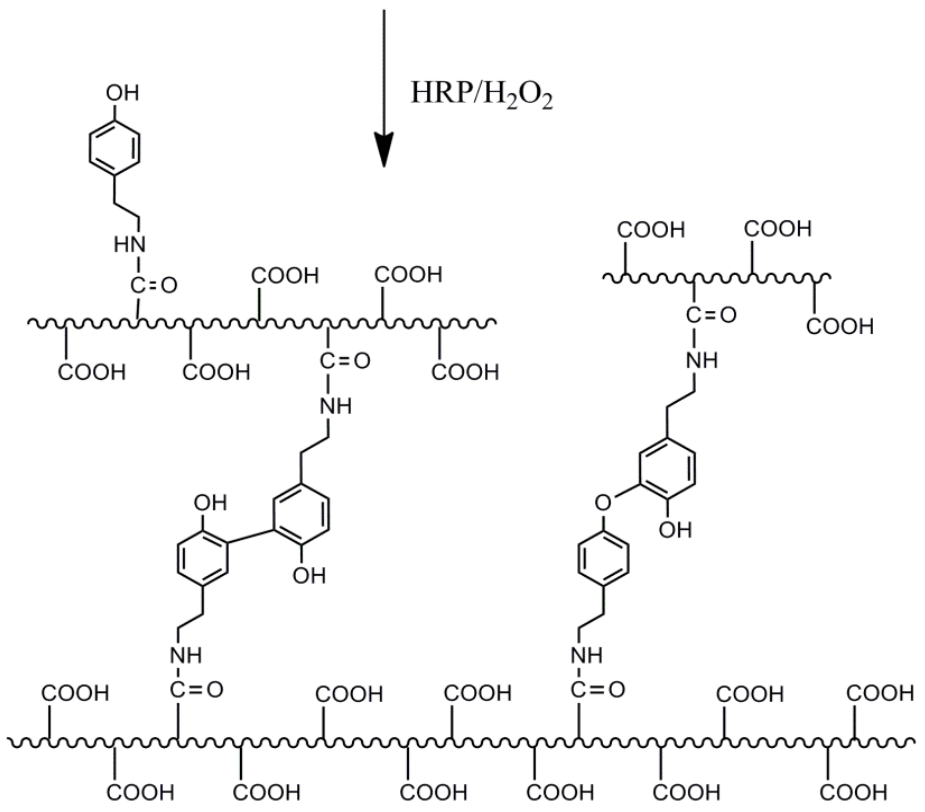

Figure 1-10. Enzymatically crosslinking tyramine grafted gelatin or hyaluronic acid chains with $\mathrm{HRP} / \mathrm{H}_{2} \mathrm{O}_{2}$ [136].

Transglutaminase and tyrosinase are other enzymes that have been used as crosslinker agents of gelatin. Transglutaminase catalyzes the covalent crosslinking between a free amine group from a protein or peptide-bound lysine and the $\gamma$-carboxamide group of a protein or peptide-bound glutamine. This enzyme has been used for the crosslinking of gelatin [137], fibrin, PEG, etc. [128]. However, its main problem is that it is involved in inflammatory reactions in joint diseases and so may actually worsen the disease [128]. Tyrosinases catalyze the oxidation of phenols into activated quinones, which 
react with a hydroxyl group or amino group via a Michael-type reaction [128]. Chen et al. obtained blends of gelatin and chitosan crosslinked with tyrosinase and transglutaminase. Tyrosine crosslinking reaction was faster, however the hydrogels obtained were weaker and the tyrosinase crosslinking of gelatin was not possible without the presence of chitosan [138]. In comparison with transglutaminase and tyrosinase, peroxidases produce a faster gelation in only a few seconds [128]. The integration of the injectable hydrogels with the host tissue in some cases may even be so good that they crosslink with extracellular matrix fibers [139], [140], enhancing the transmission of loads from the host tissue to the implanted hydrogel.

Despite the benefits of gelatin in situ formed hydrogels, their mechanical properties are rather poor, showing values of shear storage modulus lower than $2.75 \mathrm{kPa}$ for concentrated gels of $12.5 \%$ $\mathrm{w} / \mathrm{v}$ [141]. There is therefore a need to develop systems with improved mechanical properties without compromising cellular viability.

\subsection{Hydrogel reinforcement}

Typical ways of improving the mechanical properties of hydrogels consist of increasing crosslinking density or polymer concentration. However, these strategies are not so good for the encapsulation of cells or for tissue engineering as they compromise the permeability, hydrophilicity and transparency of the material and hinder cell viability [142]-[144].

Alternative strategies need to be found to improve hydrogel toughness without compromising their use in tissue engineering and cell encapsulation. Composite hydrogels filled with fibers, textiles or microparticles, hard solid structures within an embedded hydrogel, multi-layered designs, three-dimensional woven composite materials and the combination with a harder hydrogel or polymer (e.g. forming 
a secondary interpenetrated polymeric network or copolymerization) have been proposed in the literature as possible options [62], [122], [145]-[155].

In the present work two approaches were developed to improve hydrogels' mechanical properties. One was the synthesis of Interpenetrated Polymer Networks and the other the synthesis of a composite hydrogel filled with microfibers.

\subsubsection{Hydrogels reinforced by Interpenetrating Polymer Networks}

Interpenetrated Polymer Networks (IPNs) are formed by a combination of two or more polymeric networks, which spatially interpenetrate without crosslinking with each other. The networks cannot be separated unless the chemical bonds are broken. IPNs can be classified into two groups according to the chemical process followed for their synthesis (see Figure 1-11):

A) Simultaneous IPNs are obtained when polymerization precursors (monomers or oligomers and cross-linker agents) of both networks are mixed in one step, and polymerization and crosslinking of both networks occurs at the same time by independent, noninterfering chemical routes, Figure 1-11a .

B) Sequential IPNs are produced in two steps. In the first, polymerization of the first network occurs. Afterwards, this first network is swollen in the precursor solution of the second network (monomer, initiator and with or without cross-linker) (see Figure 1-11b). If a cross-linker is present a fully-IPN is obtained, while in the absence of a cross-linker, a network having linear polymers embedded within the first network is formed (semi-IPN). When a linear polymer is entrapped in a matrix, forming a semi-IPN hydrogel, a selective cross-linking of the linear polymer chains will result in obtaining a fully-IPN (see Figure 111c) [156]. 

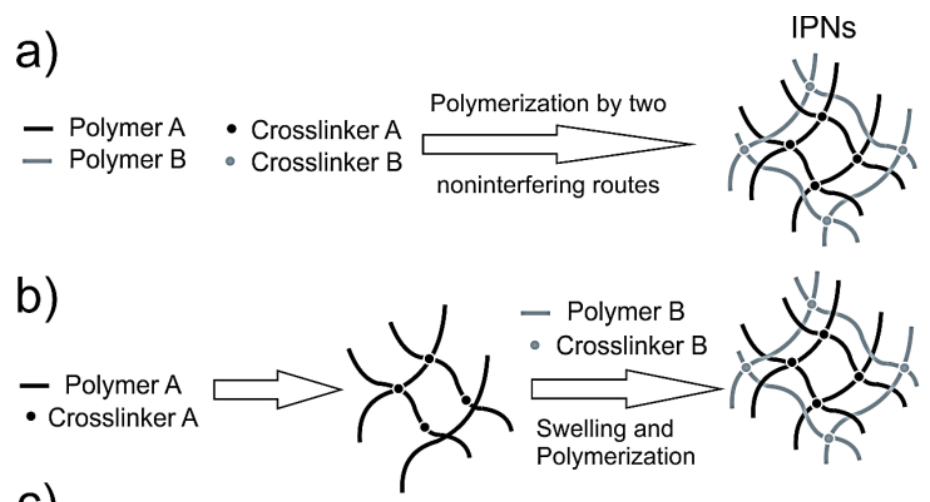

C)
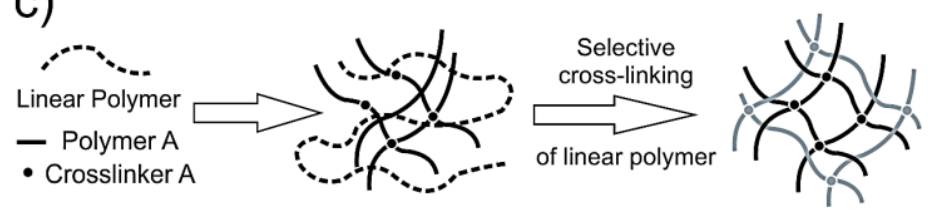

Figure 1-11. Steps needed for the synthesis of IPN formation. a) Simultaneous polymerization, b) sequential IPNs and c) selective cross-linking of a linear polymer entrapped in semi- IPN.

Gong et al. synthesized mechanically enhanced IPN hydrogels, also known as "double networks" [157]. They consist of the preparation of a densely cross-linked ionic hydrogel and a second network with a molar ratio (network 1/network 2 ) of 1-20. The hydrogels obtained were characterized by high resistance to wear and high fracture strength [158].

The miscibility of the interpenetrating polymers is an important parameter that must be considered in their production process. Polymers usually do not mix well with each other, forming a phase separated system at the nanoscale level. In order to avoid phase separation a forced compatibility can be obtained by high crosslinking [144].

Polymerization of sequential IPNs is produced in several steps. At the beginning, monomer II is soluble in the already crosslinked polymer I. Once the polymerization starts, the synthesized oligomer II will also be soluble in the first network, in which gives an optically transparent 
polymeric mixture. As polymerization proceeds, in the second step the polymeric solution loses its transparency becoming more whitish, indicating phase separation.

The IPN morphology will thus depend on the level of crosslinking of the first and the second networks, and on the procedure followed for its synthesis [159]; it is not the same to perform polymerization simultaneously as to do it sequentially. In the case of sequential IPNs, if polymers I and II are immiscible and the crosslinking density of polymer $I$ is low, i.e. the quantity of monomeric units between crosslinking points is high, then, during the polymerization of network II, the growing polymeric chains of polymer II will push away the already formed polymeric chains of I, obtaining a phase separated IPN. However, if the crosslinking density of I is high, the polymeric chains of II will interpenetrate with the chains of polymer I, and a homogenous IPN will be obtained due to the forced compatibility between both polymers [160].

\subsubsection{Hydrogels reinforced by combination with microfibers}

The use of composites made of short or loose microfibers embedded in a soft natural polymeric matrix arose from the need to improve the hydrogels' mechanical properties without increasing crosslinking density or compromising cell viability. Moreover, the microfibers should not limit hydrogel injectability, so good dispersion is needed in order to avoid needle blockage.

The term usually used to classify fibers into short micrometer or submicrometer fibers is their aspect ratio (length/diameter). The fibers should have an aspect ratio (AR) lower than 200, with diameters of a few nanometers and a length of the order of microns. Eichhorn et al. studied the effect of cellulose nanofibers in a polypropylene matrix and determined that AR smaller than 10 would not provide any major benefits over conventional micron-sized filaments and that only 
nanofibers with AR higher than 50 can guarantee a reinforcement effect [161].

An important factor that needs to be taken into account is the adhesion between the fiber and the matrix, since some micromechanical events, such as variations in stress distribution along the fiber-matrix interphase and end effects play a significant role [162], [163].

Few studies can be found in the literature on the production of short, non-aggregated or loose microfibers of biocompatible polymers for injection with hydrogel solutions, as this is a newly opened field. Short microfibers have been obtained by precipitation [164], postprocessing electrospun meshes (e.g. milling [165] or ultrasonication [166]) or direct electrospinning of short microfibers on the collector [167]. In the latter case, loose microfibers can be obtained by varying the polymer-solvent system, polymer concentration and molecular weight. Low molecular weights are usually needed to prevent chain entanglements, which lead to jet breakage and short fibers.

Coburn et al. obtained low-density electrospinning microfiber meshes of polyvinyl alcohol and chitosan that allowed cell infiltration [168], but this type of fibers could not be used in injectable hydrogels. Regev et al. obtained serum albumin short electrospun fibers; for this they used a serum albumin solution with a viscosity that enables jet fragmentation during the electrospinning process. The fibers obtained could be dispersed in the gelatin precursor solution and finally crosslinked after injection [150]. Palma et al. obtained silk nanofibers using cotton as a template that was pyrolysed to finally obtain the fibers [169], but this method can only be used with materials capable of supporting high temperatures, which is not usually the case with biodegradable polymers. Finally, Hsieh et al. obtained short fibers capable of being used in injectable hydrogels by sonicating electrospun poly(3-caprolactone-co-D,Llactide) and collagen meshes [170]. 
Different mathematical models have been proposed in the literature to predict values of the composite modulus. The application of models can help us to better understand the relationship between composite microstructure (e.g. fiber orientation, fiber-matrix interaction, etc.) and how this influences the material properties.

\section{Mathematical modeling}

1) Rule of mixtures (ROM): this is the simplest model that can be applied to predict the elastic modulus of the composite material. The elastic modulus $\left(E_{1}\right)$ is calculated assuming that the matrix and the fibers experience the same strain $\left(\varepsilon_{1}\right)$. The strain is produced by a uniform stress $\left(\sigma_{1}\right)$ applied over a uniform cross-section with area $\mathrm{A}$. The ROM equation for the apparent Young's modulus in the direction of the fibers, i.e. in the longitudinal direction of the composite, is obtained with Equation 1 [171]:

$$
E_{1}=E_{F} V_{F}+E_{M} V_{M}
$$

where $E_{F}$ and $V_{F}$ are the modulus and the volume fraction of the fibers and $E_{M}$ and $V_{M}$ are the modulus and the volume fraction of the polymeric matrix. This model works well in the case of aligned continuous fiber composites where the hypothesis of equal strain in both components is correct.

2) Inverse rule of mixtures: this is used to predict the elastic modulus $\left(E_{2}\right)$ when the force is applied perpendicular to the composite surface, i.e. in the transverse direction. In this model, it is assumed that the transverse stress is the same in the fiber and the matrix (Reuss' assumption). The inverse rule of mixtures is shown in Equation 2 [171]:

$$
E_{2}=\frac{E_{F} E_{M}}{V_{M} E_{F}+V_{F} E_{M}} .
$$

3) Halpin-Tsai equation: this model predicts all the elastic constants of short-fiber composite materials as a function of the aspect ratio of the fibers and the volume fraction and the moduli of the fibers and 
the matrix. In this model, no fiber-fiber interactions are considered. The predicted composite modulus $\left(E_{C}\right)$ can be obtained using Equation 3 [161]:

$$
E_{C}=E_{M}\left(\frac{1+\xi \eta V_{F}}{1-\eta V_{F}}\right)
$$

The parameter $\eta$ is calculated from Equation 4:

$$
\eta=\frac{\left(E_{F} / E_{M}\right)-1}{\left(E_{F} / E_{M}\right)+\xi}
$$

where $\xi$ is a shape fitting parameter that takes into account the geometry and the packing arrangement of the fibers. Different empirical equations for the calculation of $\xi$ are available and depend on the shape and on the modulus to be predicted, and usually take into account the length and the diameter of the fibers [172].

4) Shear lag theory: in this theory, the applied load is transferred from matrix to fibers by interfacial shear stresses, assuming zero tensile stress at the ends of the fibers. The shear-lag equation for the composite is, see Equation 5 [172]:

$$
E_{C}=\eta_{1} v_{f} E_{f}+\left(1-v_{f}\right) E_{m},
$$

where $E_{C}$ is the Young's modulus of the composite, $\eta_{1}$ is a lengthdependent "efficiency factor", $v_{f}$ is the volume fraction of the fibers, $E_{f}$ is the Young's modulus of the fibers and $E_{m}$ the Young's modulus of the polymeric matrix. The equation used to calculate $\eta_{1}$ (Equation $6)$ is:

$$
\eta_{1}=\left[1-\frac{\tanh (\beta L / 2)}{(\beta L / 2)}\right],
$$

being $L$ the average fiber length and $\beta$ is calculated by Equation 7:

$$
\beta^{2}=\frac{H}{\pi r_{f}^{2} E_{f}} .
$$


Here, $r_{f}$ is the fiber radius and the coefficient $H$ is calculated from Equation 8:

$$
H=\frac{2 \pi G_{m}}{\ln \left(R / r_{f}\right)},
$$

where $G_{m}$ is the matrix shear modulus and $\mathrm{R}$ is the radius of the matrix cylinder, the matrix that surrounds each fiber. Theoretical values of $R$ have been established assuming different fiber packaging models (e.g. hexagonal, square or Cox models) [172]. 


\section{Chapter 2 Objectives}



As has been previously stated, articular cartilage is an avascular tissue where cells cannot migrate to properly repair any damage; thus, there is the need to obtain a scaffold that can properly stimulate chondrocytes for the synthesis of hyaline cartilage and the proper regeneration of articular cartilage. Until now, many approaches had been studied for its regeneration but they could only obtain a fibrocartilaginous type cartilage or a mixture of fibrocartilage and hyaline cartilage which finally led to scaffold failure and the progression of cartilage degeneration.

In this thesis we hypothesized that the use of natural injectable hydrogels capable of crosslinking in the place of the defect adopting its shape and with minimal invasive surgery can be promising materials for the regeneration of articular cartilage. Following this new approach we aimed at synthesizing two new families of injectable hydrogels based on the natural protein gelatin. The first family was based on gelatin with reinforcing loose microfibers and the second family was based on a combination of gelatin and hyaluronic acid which better mimics articular cartilage composition.

Moreover, a series of synthetic IPNs that combine a hydrophobic and a hydrophilic polymer were synthesized as suitable materials with similar mechanical and permeability properties as articular cartilage host tissue. The material which better fits articular cartilage characteristics will be used as a bioreactor platform where the different scaffolds can be tested in an environment which better mimics the mechanical stresses that occur in the joints than actual bioreactors or in vitro cell cultures.

Therefore, the main objectives of this thesis were:

1) The study of the reinforcement effect of two different types of fibers, with different shape and size distribution, in the mechanical properties of the composite. Moreover, matrix-fiber interphase was 
improved with the grafting of hydrophilic polymer chains and the improvement of the mechanical properties was assessed.

2) The mechanical and biological evaluation of injectable gelatinhyaluronic acid hydrogels which better mimics articular cartilage composition. Cell morphology in the different compositions synthesized and the influence in hMSCs differentiation to a cartilage phenotype was studied.

3) Development of a non-degradable platform for a bioreactor that simulates the mechanical stresses that occur in the joints. The permeability and the compression Young's modulus was obtained for each composition and compared with typical values for articular cartilage in order to select the composition that fits the requirements. 


\section{Chapter 3}

\section{Injectable gelatin-fiber hydrogels}





\subsection{Summary*}

In this chapter injectable composites composed of gelatin hydrogels containing loose poly(L-lactide acid) (PLLA) microfibers were synthesized with the aim of improving gelatin mechanical properties for application in cartilage tissue engineering.

First, the advantages of using two different types of PLLA fibers synthesized by two different new methods were explored. In the first method, a PLLA electrospun mesh was obtained and subsequently milled to produce loose microfibers. In the second technique, the PLLA solution was projected into a rapidly stirred ethanol medium and loose microfibers were also obtained. Different proportions of both types of microfiber were mixed with solutions of tyraminegelatin derivatives to obtain the injectable composites. Hydrogel composites were subsequently prepared by enzymatic crosslinking of these solutions in the presence of $\mathrm{HRP}$ and $\mathrm{H}_{2} \mathrm{O}_{2}$. Classical preformed composites crosslinked with glutaraldehyde (GTA) were employed as comparison systems.

The interaction between the composite matrix and the hydrophobic fibers in these systems will be mainly due to the van der Waals forces between the hydroxyl and carboxylic groups of PLLA chain ends with the hydroxyl, carboxylic and amine groups of gelatin, and will probably give weak fiber-matrix interactions. PLLA was selected for its high tensile strength, low elongation and high elastic modulus. It is frequently used for cell culture, tissue regeneration and orthopedic implants due to its non-toxicity, good biocompatibility and biodegradability [173], [174].

The main purpose of the first part of this chapter is to obtain short length PLLA microfibers to be included in the injectable hydrogel precursor solution.

In the second part of the chapter, we selected one type of loose PLLA microfibers and modified their surfaces by grafting on hydrophilic 
moieties. The aim was to improve the interaction between the fibers and the gelatin matrix in order to obtain composites with better mechanical properties than those with non-modified fibers. The hydrophilic and biocompatible polymer Poly(2-hydroxyethyl methacrylate) (PHEMA) [22], [46], [49], [175]-[177] was graft polymerized onto the PLLA fibers.

The resulting composites were also injectable. They combine three kinds of macromolecules with different in vivo degradation rates. Gelatin acts as scaffold in the first stages of regeneration, retaining encapsulated cells and agglutinating the fibers. The mechanical properties of the scaffold in the early stages of implantation are very important, and it is precisely in this first stage when the role of the PHEMA grafting onto the PLLA fibers is essential. In few days, cells will secrete their own extracellular matrix (ECM) and this new matrix will play the role of the gelatin that has already been degraded or highly degraded. As this first regenerated matrix is still not mature, the remaining PLLA fibers will provide mechanical support to the new ECM. As the tissue matures and more ECM is secreted, the fibers will slowly degrade and will not compromise the mechanical stability of the defect.

*The results presented in this chapter have been published in:

Poveda-Reyes S., Mellera-Oglialoro L. R., Martínez-Haya R., GamboaMartínez T. C., Gómez Ribelles J. L. and Gallego Ferrer G. Reinforcing an Injectable Gelatin Hydrogel with PLLA Microfibers: Two Routes for Short Fiber Production. Macromol. Mater. Eng. 300(10), 977-988 (2015).

Poveda-Reyes S., Rodrigo-Navarro A., Gamboa-Martínez T.C., Rodíguez-Cabello J.C., Quintanilla-Sierra L., Edlund U., Gallego Ferrer. $G$. Injectable composites of loose microfibers and gelatin with improved interfacial interaction for soft tissue engineering. Polymer, 74, 224-234 (2015). 


\subsection{Interaction of two different types of PLLA fibers with gelatin. Two routes for short fiber production.}

\subsubsection{Materials and methods}

\subsubsection{Materials}

Gelatin (for analysis and bacteriology), dioxane (extra pure, stabilized with $2.5 \mathrm{ppm}$ of 2,6-Di-tert-butyl-4-methylphenol (BHT)), ethanol absolute (synthesis grade), sodium chloride (synthesis grade), potassium dihydrogen phosphate (extra pure), N,Ndimethylformamide (synthesis grade) and glutaraldehyde $(25 \% \mathrm{w} / \mathrm{w}$, extra pure) (GTA) were purchased from Scharlab, Barcelona, Spain. Poly(L-lactic acid) (PLLA) Ingeo 4042D (number average molecular weight of $200000 \mathrm{~g} / \mathrm{mol}, 92 \%$ L-lactide and $8 \%$ D-lactide units [178]) was supplied by Natureworks, Savage, MN, USA. Hydrogen peroxide solution $\left(30 \% \mathrm{w} / \mathrm{w}\right.$ in $\mathrm{H}_{2} \mathrm{O}$, with stabilizer), peroxidase from horseradish (HRP) type $\mathrm{VI}$, tyramine hydrochloride ( $\geq 98 \%)$, 2-(NMorpholino)ethanesulfonic acid (>99\%, MES), N-Hydroxysuccinimide (98\%), dialysis tubing (12400 MWCO), sodium azide ( $\geq 99.5 \%$, ReagentPlus ${ }^{\circledR}$ ), potassium chloride (for molecular biology), 4-(2hydroxyethyl) piperazine 1-ethanesulphonic acid (HEPES), dichloromethane (anhydrous, $299.8 \%$ ) and Dulbecco's phosphate buffered saline (DPBS) solution were provided by Sigma-Aldrich, Germany.

$\mathrm{N}$-(3-Dimethylaminopropyl)-N'-ethylcarbodiimidehydrochloride (EDC) was provided by Iris Biotech $\mathrm{GmbH}$, Marktredwitz, Germany. Calcium chloride anhydrous was provided by Panreac, Barcelona, Spain.

For the cell culture tests, mouse fibroblasts L929, bovine serum albumin (BSA), formalin 10\% and Triton X-100 Bioxtra were purchased from Sigma-Aldrich. Dulbecco's Modified Eagle Medium (DMEM) high glucose, fetal bovine serum (FBS), live/dead kit for mammalian cells and BODIPY-FL phallacidin (1:100) were provided by Life Technologies SA, Madrid, Spain. Penicillin/streptomycin and L- 
glutamine were purchased from Lonza, Spain. MTS (3-(4,5dimethylthiazol-2-yl)-5-(3-carboxymethoxyphenyl)-2-(4-sulfophenyl)$2 \mathrm{H}$-tetrazolium) was provided by Promega, Spain. Vectashield with incorporated 4',6-diamidino-2-phenylindole (DAPI) was purchased from Vector Laboratories, Peterborough, UK.

Krebs Ringer Buffer (KRB) solution was prepared with $115 \mathrm{mM}$ sodium chloride, $5 \mathrm{mM}$ potassium chloride, $1 \mathrm{mM}$ calcium chloride, 1 $\mathrm{mM}$ potassium dihydrogen phosphate and $25 \mathrm{mM}$ HEPES.

\subsubsection{Preparation of PLLA microfibers}

\section{Electrospinning produced microfibers}

$10 \% \mathrm{w} / \mathrm{v}$ PLLA solution at a volume ratio of dichloromethane to dimethyl formamide $70 / 30$ was projected through a $0.45 \mathrm{~mm}$ diameter needle in a $5 \mathrm{~mL}$ syringe. A syringe pump was used to feed the polymer solution at a rate of $4 \mathrm{~mL} / \mathrm{h}$. A high-voltage power supply was employed to generate the electric field, applying a voltage of 20 $\mathrm{kV}$ between the needle and the collector plate. The tip-to-collector distance was fixed at $17 \mathrm{~cm}$ and the electrospun fibers were collected on aluminum foil. After electrospinning, the mesh was left at room temperature for $24 \mathrm{~h}$ to evaporate the residual solvents.

In order to obtain loose fibers we developed the following protocol. First, both sides of the electrospun mesh were treated with air plasma (Plasma treatment unit PICCOLO by Plasma Electronic GmbH, Neuenburg, Germany) at $50 \mathrm{~Pa}$ and $150 \mathrm{~W}$ for $30 \mathrm{~s}$ for a $65 \mathrm{mg}$ mesh. Then small pieces of the electrospun mesh were embedded in water and converted into ice cubes. The ice cubes were slightly ground up in small pieces, immersed in liquid nitrogen and finely ground with a $600 \mathrm{~W}$ grinder for $20 \mathrm{~min}$. The obtained powdered ice was melted at room temperature and sieved, collecting the PLLA electrospun fibers (PLLA-ES) between 80 and $30 \mu \mathrm{m}$ with a nylon cloth, which were subsequently dried by lyophilization. 
The loose dried microfibers were subjected to plasma air treatment for $5 \mathrm{~min}$ at $50 \mathrm{~Pa}$ and $300 \mathrm{~W}$ to enhance their wettability prior to using them in the composite preparation.

\section{Microfibers produced by turbulent flow}

PLLA high turbulent flow microfibers (PLLA-HT) were obtained from a $5 \% \mathrm{w} / \mathrm{v}$ PLLA solution in dioxane. $5 \mathrm{~mL}$ of PLLA solution was injected at a rate of $34 \mathrm{~mL} / \mathrm{min}$ using a syringe pump through a $0.9 \mathrm{~mm}$ diameter needle in a high turbulent cold ethanol bath at $-20^{\circ} \mathrm{C}$. The bath and projected solution were constantly stirred at $15000 \mathrm{rpm}$ using the IKA T25 digital ULTRA-TURRAX mixer (Staufen, Germany) for a total time of $2 \mathrm{~min}$.

After fiber precipitation and in order to eliminate the dioxane, the fibers were washed several times with ethanol. Then two exchanges drop by drop with water under stirring were carried out to avoid fiber agglomeration due to the ethanol. Afterwards, the PLLA-HT fibers were sieved, lyophilized and treated with plasma prior to using them in the composite preparation with the same conditions as those described in the previous method.

\subsubsection{Preparation of injectable composite hydrogels}

Before preparing the composites, the gelatin was modified in order to introduce phenol groups able to crosslink gelatin in the presence of $\mathrm{HRP}$ and $\mathrm{H}_{2} \mathrm{O}_{2}$. To do this, the carboxylic groups of gelatin were bound with the amine groups of tyramine as explained by Sakai et al. [129]. In short, $2 \% \mathrm{w} / \mathrm{v}$ gelatin in $50 \mathrm{mM}$ MES was dissolved at $60^{\circ} \mathrm{C}$ for $30 \mathrm{~min}$ under stirring ( $0.4 \mathrm{~g}$ of gelatin and $0.1952 \mathrm{~g}$ of MES in 20 $\mathrm{mL}$ ). Then $0.2 \mathrm{~g}$ of tyramine hydrochloride was added and the mixture was stirred for $20 \mathrm{~min}$ at room temperature. The $\mathrm{pH}$ was adjusted to 6 and $0.0189 \mathrm{~g}$ NHS was added and the mixture was stirred for 30 min to obtain good homogenization. Afterwards, 0.144 $\mathrm{g}$ of EDC was added and stirred for $18 \mathrm{~h}$. The non-reacted products were removed dialyzing against deionized water for $48 \mathrm{~h}$. The modified gelatin was lyophilized for further use. 
To determine the quantity of phenol $(\mathrm{Ph})$ groups introduced in the gelatin, the absorbance of $0.1 \% \mathrm{w} / \mathrm{w}$ aqueous solution of the grafted gelatin was measured at $275 \mathrm{~nm}$ with the CECIL CE9200 UV/VIS double beam spectrophotometer (Buck Scientific, Norwalk, USA), obtaining a value of $1.9 \times 10^{-7}$ moles of tyramine $\mathrm{mg}^{-1}$ of gelatin. The content of introduced $\mathrm{Ph}$ groups was calculated from a calibration curve of known percentages of tyramine hydrochloride in distilled water.

To obtain the composites from the tyramine grafted gelatin (GEL-TA) with PLLA microfibers (obtained by electrospinning or by the high turbulent bath) different quantities of fibers $(0,0.5,1$ and $1.5 \% \mathrm{w} / \mathrm{v}$, higher fiber concentrations start producing fiber aggregates limiting their injectability) were dispersed in $80 \%$ of the total volume of composite in Krebs Ringer Buffer (KRB) and subjected to an ultrasonic treatment ( $30 \mathrm{~W}, 0.4 \mathrm{~s}$ on and $0.2 \mathrm{~s}$ off, Bandelin Sonopuls, Berlin, Germany) for $2 \mathrm{~min}$. After that, a $5 \% \mathrm{w} / \mathrm{v}$ of tyramine grafted gelatin was fully dissolved in the fiber solution at $37^{\circ} \mathrm{C}$. Finally, the crosslinking of the GEL-TA with different fiber content was carried out using a $10 \% \mathrm{v} / \mathrm{v}$ of $12.5 \mathrm{U} / \mathrm{mL} \mathrm{HRP}$ and $10 \% \mathrm{v} / \mathrm{v}$ of $20 \mathrm{mM} \mathrm{H}_{2} \mathrm{O}_{2}$ (1.25 U m/L and $2 \mathrm{mM}$ concentration in the final volume, respectively). Cylindrical samples with a diameter of $8 \mathrm{~mm}$ and $2 \mathrm{~mm}$ high were obtained by injecting a total volume of $300 \mu \mathrm{L}$ into the wells of a cell culture plate. Hereafter, the composites will be named as GEL-TA xPLLA-HT or GEL-TA xPLLA-ES (where $x$ is the percentage of fibers in the composite) for the composites with the high turbulence microfibers or the electrospun ones, respectively.

\subsubsection{Preparation of preformed composite hydrogels}

Previous to preparing the composites, bare gelatin was dissolved in DPBS at $60^{\circ} \mathrm{C}$ at a concentration of $6.25 \% \mathrm{w} / \mathrm{v} .2 \mathrm{~mL}$ of gelatin solution was mixed with four different concentrations of plasmatreated PLLA-HT microfibers $(0,0.5,1$ and $1.5 \% \mathrm{w} / \mathrm{v}$, referred to the total volume of the composite). These mixtures were subjected to ultrasonic treatment ( $30 \mathrm{~W}, 0.4 \mathrm{~s}$ on and $0.2 \mathrm{~s}$ off) for $2 \mathrm{~min}$ and 
placed into a $30 \mathrm{~mm}$ diameter Petri dish. Subsequently they were crosslinked with $0.4 \mathrm{~mL}$ of the GTA aqueous solution for $45 \mathrm{~min}$ in an orbital shaker at $150 \mathrm{rpm}$ to obtain composites containing $5 \% \mathrm{w} / \mathrm{v}$ gelatin and $0.5 \% \mathrm{v} / \mathrm{v}$ GTA. The volume of solutions was calculated to obtain samples with a thickness similar to that of the injectable systems $(2 \mathrm{~mm})$. The composites were punched to obtain cylinders of $8 \mathrm{~mm}$ in diameter that were then immersed in a $0.1 \mathrm{M}$ glycine solution at $37^{\circ} \mathrm{C}$ for $1 \mathrm{~h}$ to block residual aldehyde groups of GTA. Finally, the samples were washed three times with DPBS solution [150], [151]. Hereafter, the composites will be named as GEL-GTA $x$ PLLA-HT (where $x$ is the percentage of fibers in the composite).

\subsubsection{Morphology and microstructure of the fibers and composites}

Fibers and composites were macroscopically characterized by observation in a Leica MZ APO stereomicroscope (Germany). They were microscopically characterized with a Scanning Electron Microscope (JEOL JSM-5410, Japan) (SEM) equipped with a cryo-unit Oxford CT 1500. To observe the samples in the hydrogel state, swollen samples were frozen, cryofractured and dried at $-90^{\circ} \mathrm{C}$ in the cryounit for $45 \mathrm{~min}$. Then, the samples' surface was gold sputtered and observed at an accelerating voltage of $10 \mathrm{kV}$ and $15 \mathrm{~mm}$ working distance.

\subsubsection{Compression test}

Unconfined compression tests were performed on at least five replicates of the swollen composites of each composition immersed in DPBS with $0.02 \%$ sodium azide at room temperature. Sample dimensions were $8 \mathrm{~mm}$ diameter and $2 \mathrm{~mm}$ thick, and were tested at a rate of $50 \mu \mathrm{m} / \mathrm{min}$ on a thermomechanical analyzer (EXSTAR $\mathrm{TMA} / \mathrm{ss} 6000$, Seiko Instruments Inc, Japan). 2\% Pre-deformation was applied to the samples followed by the total compression. Young's modulus was calculated from the slope in the linear region corresponding to $2-20 \%$ strain. 


\subsubsection{Swelling test}

After preparing the composites, the samples were washed and maintained in DPBS with $0.02 \% \mathrm{w} / \mathrm{v}$ sodium azide until equilibrium was reached; sodium azide was added as a biocide to prevent bacterial growth. Then, they were weighed to obtain their swollen mass $\left(m_{s}\right)$ and lyophilized to obtain their dry mass $\left(m_{d}\right)$. Water reabsorption capacities of the composites were also determined by soaking the lyophilized samples in DPBS solution with $0.02 \%$ sodium azide at $37^{\circ} \mathrm{C}$ until equilibrium was reached, $72 \mathrm{~h}$. Gelatin water content in both experiments $\left(w^{\prime}(\%)\right)$ was calculated from the Equation 9, where $m_{\text {gelatin }}$ is the dry mass of gelatin in the composites:

$$
w^{\prime}(\%)=\frac{m_{s}-m_{d}}{m_{\text {gelatin }}} \cdot 100 .
$$

At least five replicates per sample were used for the experiments.

\subsubsection{Cell encapsulation and culture}

Mouse fibroblasts 1929 were expanded in $75 \mathrm{~cm}^{2}$ flasks and incubated in a $5 \% \mathrm{CO}_{2}$ humidified atmosphere at $37 \stackrel{\circ}{\circ} \mathrm{C}$ until confluence. DMEM with $4.5 \mathrm{~g} / \mathrm{L}$ glucose supplemented with $10 \% \mathrm{FBS}$, $1 \%$ penicillin/streptomycin $(100 \mathrm{U} / \mathrm{mL} / 100 \mathrm{mg} / \mathrm{mL})$ and $1 \% \mathrm{~L}-$ glutamine, was used as culture media. After confluence, cells were harvested, counted with a hemocytometer and encapsulated in pure gelatin injectable hydrogels (GEL-TA) prepared at $3 \%(\mathrm{w} / \mathrm{v})$ and injectable gelatin composites with the same concentration of gelatin and $1 \% \mathrm{w} / \mathrm{v}$ PLLA-HT fibers.

The gelatin solution was mixed with HRP $(12.5 \mathrm{U} / \mathrm{mL})$ and sterilized by filtering with a $0.22 \mu \mathrm{m}$ filter. The quantity of gamma radiated ( 25 kGys) sterilized fibers needed per hydrogel was placed into the wells of the cell culture plate and $5 \mu \mathrm{L}$ of the filtered GEL-TA-HRP solution was added per well to keep the microfibers inside them. Then, the fibroblasts were mixed with the sterilized GEL-TA-HRP solution at a concentration of $4 \times 10^{6}$ cells $/ \mathrm{mL}$, and $40 \mu \mathrm{L}$ of this cellular suspension was then added to each well. Finally, the composites 
were formed by adding $5 \mu \mathrm{L}$ of $\mathrm{H}_{2} \mathrm{O}_{2}(20 \mathrm{mM})$ to each well. After 15 min, $1 \mathrm{~mL}$ of media was added to each well and incubated for $1,7,14$ and 21 days. The medium was renewed every 3 days.

\subsubsection{Cell viability and distribution}

The viability and distribution of cells in the injectable pure hydrogel (GEL-TA) and injectable composite (GEL-TA 1PLLA-HT) were evaluated at 1 and 10 days of culture using the Live/Dead kit for mammalian cells. At the defined time points the samples were washed with DPBS and incubated for $15 \mathrm{~min}$ at $37^{\circ} \mathrm{C}$ in DPBS with $1 \mu \mathrm{M}$ of calcein AM and $2 \mu \mathrm{M}$ ethidium homodimer-1 (EthD-1). Then, in vivo analysis of live (stained in green with calcein AM) and dead cells (stained in red with EthD-1) was assessed in the inverted fluorescence microscope Eclipse 80i (Nikon). Several images were taken from two different replicates of each sample. The resulting images were representative of the whole sample. Cell viability was confirmed using the 3-(4,5dimethylthiazol-2-yl) 5-(3-carboxymethoxyphenyl)-2-(4-sulfophenyl)$2 \mathrm{H}$-tetrazolium (MTS) assay following the manufacturer's instructions. In short, after different times of culture the samples were washed twice with DPBS and incubated with fresh culture medium with neither phenol red nor FBS but containing MTS reagent (ratio 5:1) at $37^{\circ} \mathrm{C}$ for $3 \mathrm{~h}$ in darkness. The absorbance was measured at $490 \mathrm{~nm}$ with a microplate reader Victor 3 (Perkin-Elmer, Waltham, MA, U.S.A.) after the incubation period with MTS.

\subsubsection{Cell morphology by cytoskeleton observation}

In order to examine cell morphology within the hydrogels and possible differences of cell shape due to the presence of microfibers the samples cultured for 1 day were processed for fluorescence microscopy observation. Prior to staining, cultured samples were fixed with formalin at $10 \%$ for $1 \mathrm{~h}$ and $4^{\circ} \mathrm{C}$ and then washed 3 times in DPBS. Fixed samples were left overnight in $30 \% \mathrm{w} / \mathrm{v}$ sucrose and then included in OCT to obtain sections $15 \mu \mathrm{m}$ thick in a Leica CM1520 cryotome at $-20^{\circ} \mathrm{C}$. The $15 \mu \mathrm{m}$ sections were pre-incubated 
with $0.1 \%$ Triton $\mathrm{X}-100$ in DPBS for $5 \mathrm{~min}$ at room temperature followed by DPBS washing. Afterwards, incubation with $1 \%$ bovine serum albumin (BSA) in DPBS for 30 min at room temperature was performed prior to adding BODIPY-FL phallacidin to label the actin cytoskeleton. Finally the cellular nuclei were labeled with DAPI. Several micrographs were taken of duplicate samples using an Eclipse 80i microscope.

\subsubsection{Statistical analysis}

Data are presented as means \pm standard deviation. Statistical comparisons between samples were examined using one way ANOVA where $p$ values $<0.05$ were considered significant. 


\subsubsection{Results and Discussion}

\subsubsection{Morphology and microstructure of the fibers}

The appearance of the high turbulence PLLA-HT microfibers obtained with the new method based on injecting the PLLA solution into a highly-stirred cold ethanol bath is shown in Figure 3-1. After fiber Iyophilization the microfibers agglomerate forming the cotton-like structure shown in Figure 3-1a. The subsequent treatments with plasma, immersion in water and sonication were effective in dispersing the fibers, as can be seen in the picture in Figure 3-1b. The better the distribution of the fibers in water, the better the dispersion in the aqueous gelatin solution, and the composites should be homogeneous, without presenting filler agglomerations. In the microscopic picture of PLLA-HT microfibers (Figure 3-1c), it can be seen that the size and shape of the microfibers is quite heterogeneous and their surface is quite rough. This is a positive result because with this morphology the specific surface of contact between the fibers and the hydrogel matrix is high, consequently enhancing interaction between the filler and the gelatin matrix. The diameter of the fibers obtained by this method is $0.73 \pm 0.45 \mu \mathrm{m}$ and the length is $47.09 \pm 18.96 \mu \mathrm{m}$, obtaining an average aspect ratio (AR) of 64. By contrast, the electrospun PLLA-ES microfibers present a smooth surface (see Figure 3-1d), a more homogenous diameter distribution with values of $1.15 \pm 0.29 \mu \mathrm{m}$ and a length of $45 \pm 28.37$ $\mu \mathrm{m}$, obtaining an average $A R$ of 39 . In both cases fibers have an $A R$ lower than 200, value usually used to classify the fibers into short micrometer or sub-micrometer fibers; and in the case of PLLA-HT without any extra milling process. Optical microscope images of individual PLLA-HT and PLLA-ES microfibers can be seen in Figure 3$1 \mathrm{e}$ and Figure 3-1f, respectively. 

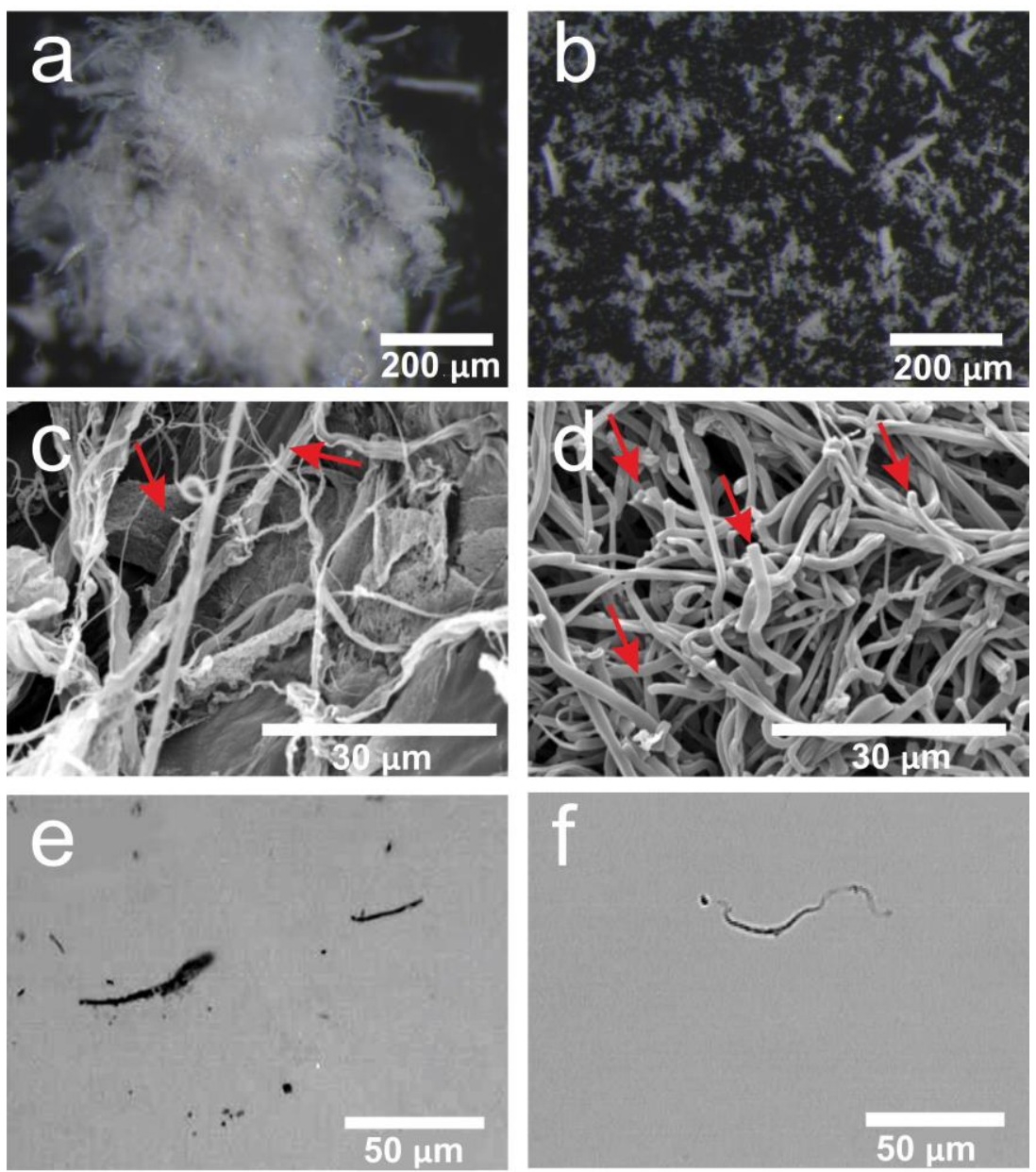

Figure 3-1. Stereomicroscope images showing (a) the cotton like appearance of the PLLA-HT fibers after being lyophilized and (b) the dispersed PLLA-HT fibers in water. SEM images of (c) high turbulence PLLA-HT fibers and (d) electrospun PLLA-ES fibers. And optical microscope images showing individual microfibers of (e) PLLA-HT and (f) PLLA-ES. Red arrows indicate the end of the fibers.

\subsubsection{Morphology and microstructure of composites}

The tyramine grafted gelatin was crosslinked by oxidizing the phenol groups in the presence of the HRP enzyme and the $\mathrm{H}_{2} \mathrm{O}_{2}$. In this reaction, the hydrogen peroxide acts as the oxidant of the HRP that 
then oxidizes two tyramines forming a covalent bond between them (see Figure 1-10). After that, the HRP returns to its original state [134]. The crosslinking reaction is very fast, it takes less than one minute, avoiding the precipitation of the fibers in the gelatin solution and enabling homogeneous distribution of fibers in the hydrogel matrix. The macroscopic aspect of the enzymatically crosslinked GELTA composites with the ultra turbulent obtained PLLA fibers (PLLA$\mathrm{HT}$ ) and the electrospun milled ones (PLLA-ES) are shown in Figure 3$2 a$ and Figure $3-2 b$, respectively. All the composites maintain the shape at physiological temperature, and those containing the highest fiber content (1.5\%) have the best handling and integrity properties. The fibers give opacity to the samples as the composites are more whitish than pure hydrogels (0\%). A higher opacity is also observed in the composites crosslinked with glutaraldehyde (GEL-GTA) in comparison to pure gelatin, which in this case is yellow due to the action of the glutaraldehyde, Figure 3-2c.

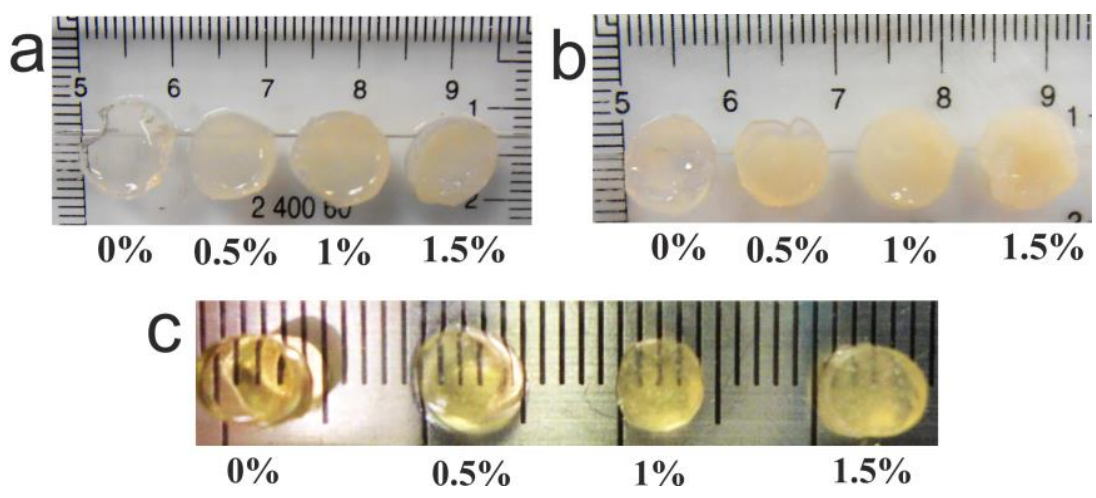

Figure 3-2. Macroscopic images of the composites with different PLLA fiber content ( $W / v$ percentages): (a) enzymatically crosslinked with embedded PLLA-HT microfibers (GEL-TA PLLA-HT), (b) enzymatically crosslinked with embedded electrospun fibers (GEL-TA PLLA-ES) and (c) chemically crosslinked with GTA and embedded PLLA-HT microfibers (GEL-GTA PLLA-HT). 

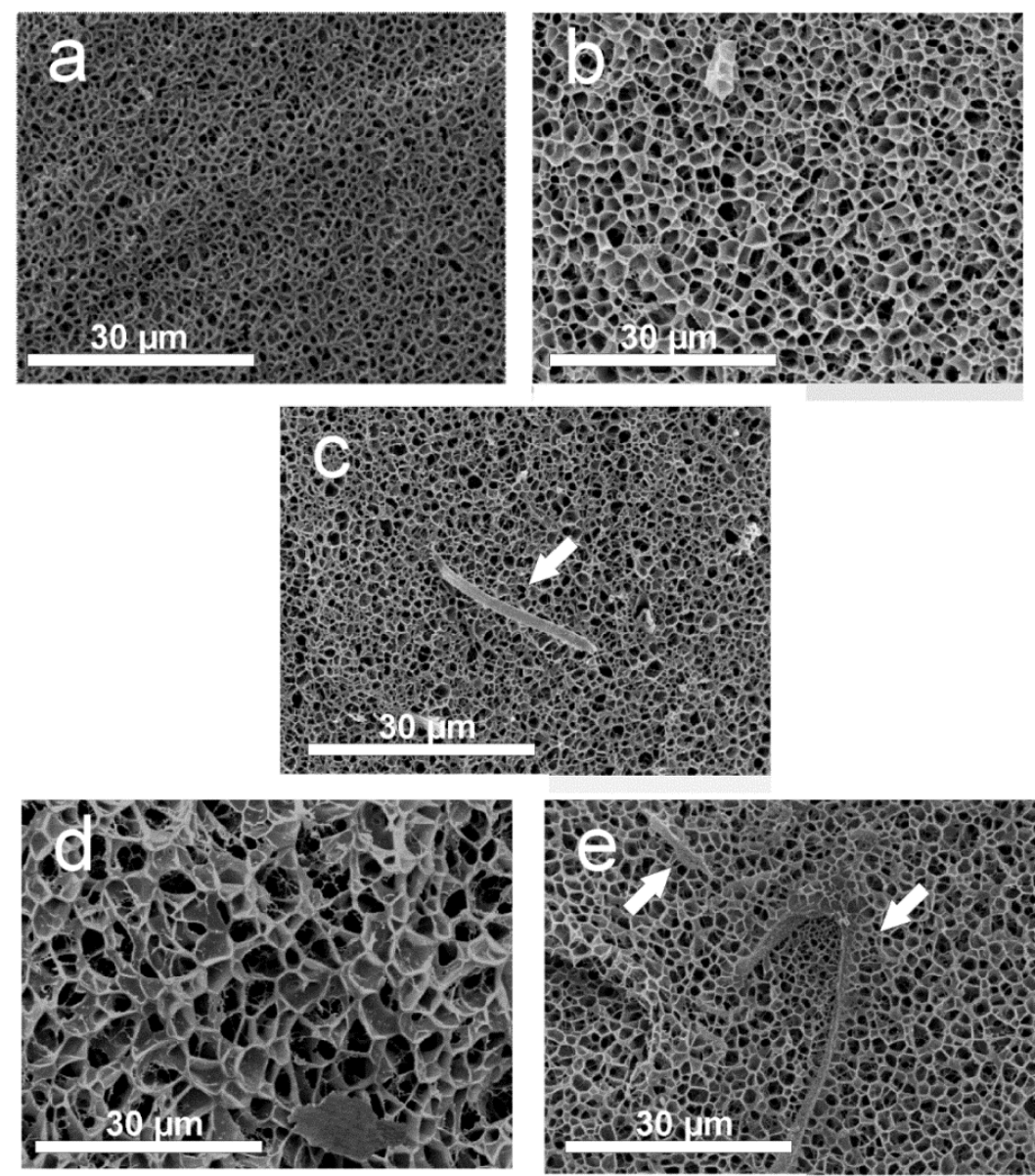

Figure 3-3. Cryo-SEM images of: (a) pure injectable gelatin (GEL-TA); (b) injectable composite with $1 \% \mathrm{w} / \mathrm{V}$ PLLA-HT fibers (GEL-TA 1PLLAHT); (c) injectable composite with $1 \% \mathrm{w} / \mathrm{V}$ PLLA-ES fibers (GEL-TA 1PLLA-ES); (d) chemically crosslinked gelatin (GEL-GTA); and (e) chemically crosslinked composite with 1\% w/V PLLA-HT fibers (GELGTA 1PLLA-HT). White arrows mark the PLLA fibers.

Figure 3-3 shows the microscopic images of composites with 0 and $1 \%$ of PLLA-HT microfibers crosslinked by enzymatic or chemical reaction, as representative of the rest of samples. Figure 3-3a and Figure 3-3b represent the cryo-SEM images of GEL-TA and GEL-TA 1PLLA-HT, respectively. In this case the obtained hydrogels have a 
pore size of 0.6-1.4 $\mu \mathrm{m}$ for GEL-TA and a wider pore size distribution was obtained for the composite with PLLA-HT fibers with 1.2-4 $\mu \mathrm{m}$. In the GEL-TA 1PLLA-HT image, the PLLA-HT fibers cannot be clearly seen, they were probably lost in the process of cutting the material. In the case of GEL-TA 1PLLA-ES (Figure 3-3c) some fibers can be seen sticking out from the microporous hydrogel with micropores of 0.64 $2 \mu \mathrm{m}$. Figures $3 \mathrm{~d}$ and $3 e$ depict pure GEL-GTA and GEL-GTA 1PLLA-HT composite observed by cryo-SEM, respectively. Here, the pore size of pure gelatin is bigger than in the case of enzimatically crosslinked gelatin, with interval dimension of 3.75-6 $\mu \mathrm{m}$ for GEL-GTA. However, when introducing the fibers the gelatin reduces the size of the pores and the pore distribution interval becomes similar to that of the injectable composite, 1.5-2 $\mu \mathrm{m}$ for the composite GEL-GTA 1PLLA-HT. Again some fibers mixed with the gelatin matrix can be seen in this composite, marked by white arrows in Figure 3-3e. This way the chemical crosslinking of gelatin in the presence of fibers results in reduction of pore size, as we can see in Figure 3-3e in comparison to Figure 3-3d. Despite these small differences in pores size, the mixture of fibers with the precursor solution of the injectable gelatin did not affect the crosslinking reaction, and we were able to prepare injectable microfiber-gelatin composites as novel materials for tissue engineering.

\subsubsection{Mechanical properties}

Figure 3-4 shows the characteristic stress-strain curves of each composite. For the glutaraldehyde crosslinked composites (Figure 34c), as the quantity of PLLA-HT fibers increases, so the stress augments at the same deformation, as observed by the upward shift of the GEL-GTA PLLA-HT curves. This effect is more notable at higher strains. The mechanical behavior of hydrogels at compression is governed by their water permeability. Water retention in the hydrogel by the polymeric chains increases the Young's modulus. This way, the effect of the PLLA hydrophobic fibers in the composites is to reduce water permeability and consequently increases the 
mechanical strength in proportion to the fibers content. Water retention is higher for high deformations because the fibers are closer and more compact hindering water flow. This would explain the more pronounced reinforcement observed at high deformations in Figure 3-4c.

No reinforcement is observed in the injectable composites containing the ultra turbulent obtained fibers (GEL-TA PLLA-HT), as seen in the stress-strain curves in Figure 3-4a. However, significant reinforcement of the injectable gelatin was obtained when the electrospinning milled fibers were used to fabricate the composites (Figure 3-4b), as is noticeable by the change in the slope, which becomes higher with the increasing fiber content. A similar enhancement of the mechanical properties has been reported by other authors in systems that combine hydrogels and fibers [147], [150], [179]-[182], although the novel side to our system is the use of short fibers capable of being dispersed in an injectable hydrogel solution. The agglomeration of the high turbulent obtained fibers in the injectable gelatin solution prior to crosslinking may be causing the null reinforcement found here. Fiber agglomerates are not a barrier to water flow, with the contribution of water to the mechanical performance of these composites being very similar to that of pure gelatin.

In order to predict the Young's modulus (E) of the PLLA fiber reinforced gelatin a shear lag model based on the rule of mixtures has been applied (see Equation 5, page 62). In this case, the matrix shear modulus, $G_{m}$, is around $200 \mathrm{~Pa}$ for gelatin and for $\mathrm{R}$ an average distance between fibers of $30 \mu \mathrm{m}$ has been assumed.

The theoretical values obtained for both types of composites (GEL-TA PLLA-HT and GEL-TA PLLA-ES) are shown in Table 3-1. 
a)

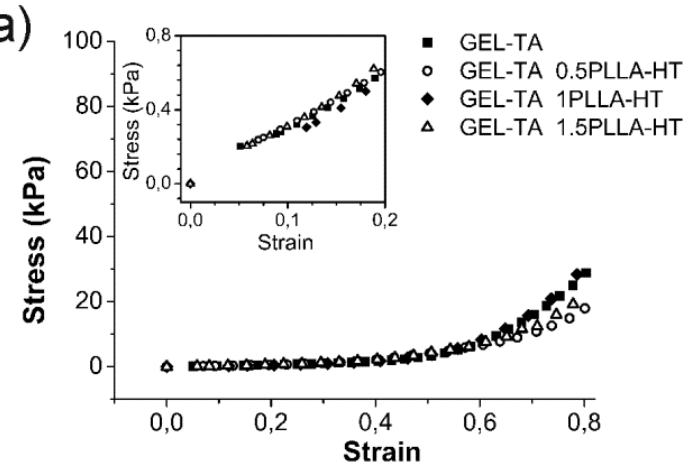

b)

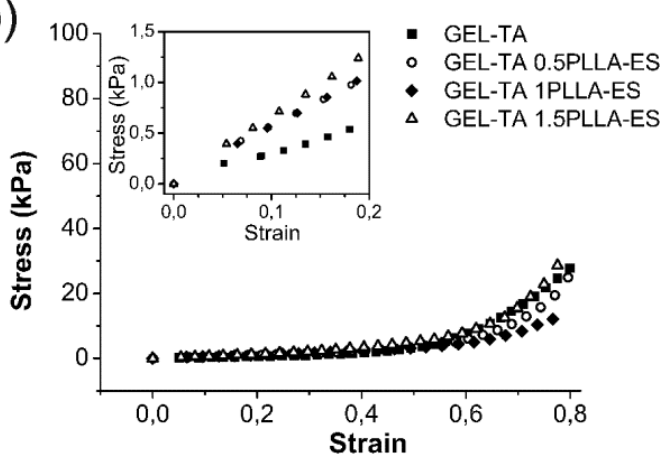

c)

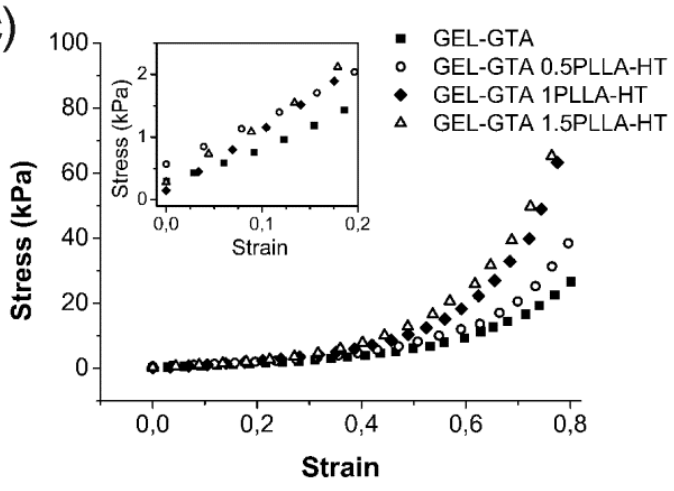

Figure 3-4. Stress-strain curves for the different composites: (a) Injectable gels with high turbulent obtained fibers (GEL-TA PLLA-HT); (b) injectable gels with elctrospinning milled fibers (GEL-TA PLLA-ES); and (c) glutaraldehyde crosslinked hydrogels with high turbulent obtained fibers (GEL-GTA PLLA-HT). The inserts show an enlargement of the curves up to $20 \%$ strain, where Young' modulus was calculated. 
Table 3-1. Theoretical Young's modulus obtained with the shear lag model, Equation 5.

\begin{tabular}{cccc}
\hline \%PLLA fibers & $\boldsymbol{v}_{\boldsymbol{f}}$ & $\begin{array}{c}\mathrm{E}_{\mathrm{C}} \text { PLLA-HT } \\
{[\mathrm{kPa}]}\end{array}$ & $\begin{array}{c}\mathrm{E}_{\mathrm{C}} \text { PLLA-ES } \\
{[\mathrm{kPa}]}\end{array}$ \\
\hline 0.5 & 0.0038 & 2.72 & 2.71 \\
1 & 0.0076 & 2.79 & 2.78 \\
1.5 & 0.0114 & 2.87 & 2.84
\end{tabular}

Experimental Young's modulus was calculated as the slope in the initial linear region from the stress-strain curves (see enlargement in Figure 3-4) where the hydrogel shows elastic behavior. The E values for all the composites are compared in Figure 3-5. For GEL-GTA composites, as mentioned previously, the presence of fibers increases the Young's modulus although the ANOVA test did not show significant differences between the composites with different fiber content. Significant differences were only obtained when the composites with fibers are compared with GEL-GTA, with an increase in $\mathrm{E}$ from $6.43 \pm 0.46 \mathrm{kPa}$ to $8.07 \pm 0.82 \mathrm{kPa}$ for GEL-GTA and the $0.5 \%$ composite, respectively. Moreover, the GEL-GTA PLLA-HT composites show higher modulus for all the compositions than the GEL-TA PLLAHT composites. No significant differences between the averages for the different GEL-TA PLLA-HT composites were obtained with the ANOVA test, so no reinforcement in compression could be achieved in the enzymatically crosslinked gelatin hydrogels with the fibers obtained by the high turbulence method. However the obtained values are similar to the ones obtained theoretically (see Table 3-1), and go from $2.95 \pm 0.29 \mathrm{kPa}$ for GEL-TA 0.5\% PLLA-HT to $3.07 \pm 0.09 \mathrm{kPa}$ for GEL-TA 1.5\% PLLA-HT, corroborating the theoretical model. By contrast, when electrospun fibers are embedded in the enzymatically crosslinked gelatin, an increase in $E$ is achieved by increasing fiber content from $2.65 \pm 0.33 \mathrm{kPa}$ for GEL-TA to $6.69 \pm 0.28 \mathrm{kPa}$ for the 
composite with $1.5 \%$ of PLLA-ES, values much higher than the ones obtained with the theoretical model (see Table 3-1). The statistical analysis shows significant differences between all the samples except between the mean of the composite with $0.5 \%$ fibers and $1 \%$ PLLA-ES fibers. Furthermore, the obtained moduli are higher than the ones corresponding to the GEL-TA PLLA-HT composites, but not as high as the ones obtained when the gelatin is chemically crosslinked with glutaraldehyde. This effect can be explained by the agglomeration of the high turbulent obtained fibers and inhomogeneous dispersion in the hydrogel solution, as has been mentioned in the previous paragraph.

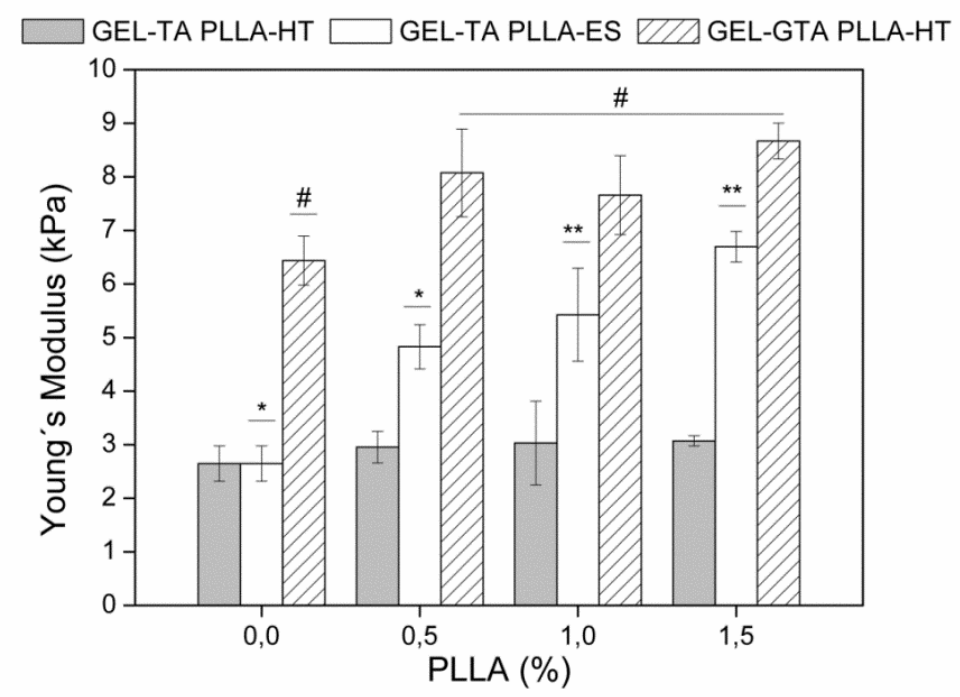

Figure 3-5. Young's modulus as a function of the PLLA fiber content for the different synthesized composites. Asterisks show the significant differences among the GEL-TA PLLA-ES composites and the dash symbols show the significant differences among the GEL-GTA PLLA-HT composites. 


\subsubsection{Equilibrium water content}

Figure 3-6a compares the percentage of swelling, referred to as the quantity of DPBS absorbed by the composite gelatin chains ( $w^{\prime}(\%)$, see Equation 9). This bar chart shows a higher swelling capacity for the hydrogels crosslinked with glutaraldehyde than for the enzymatically crosslinked ones, probably due to the different chemistries and the differences in crosslinking densities. However, the ANOVA test shows no significant differences between the different compositions of the GEL-GTA PLLA-HT samples, indicating that the different pores sizes observed in the SEM pictures Figure 3$3 d$ and Figure $3-3 e$ are not sufficient to change gelatin porosity and water sorption. For the GEL-TA gels with microfibers made by the high turbulent bath, significant differences among GEL-TA, GEL-TA 0.5PLLA-HT and GEL-TA 1PLLA-HT were found. Specifically, the swelling capacity of the composites increases with the fiber percentage. This unexpected effect is attributed to the imperfect interaction between the fibers and the gelatin matrix and the presence of gaps able to lodge higher quantities of water. The weak interaction between fibers and matrix in the GEL-TA PLLA-ES series makes significant statistical differences between the pure gelatin and the composites, while no differences were obtained when comparing different fiber contents.

Figure 3-6b compares the reabsorption capacity of the GEL-GTA and GEL-TA systems after lyophilization. In both cases, a reduction is observed compared to the initial absorption capacity of DPBS. This effect is probably due to the pores collapsing in the lyophilizing process. Pure gelatin showed the lowest reabsorption capacity compared to the composites. 

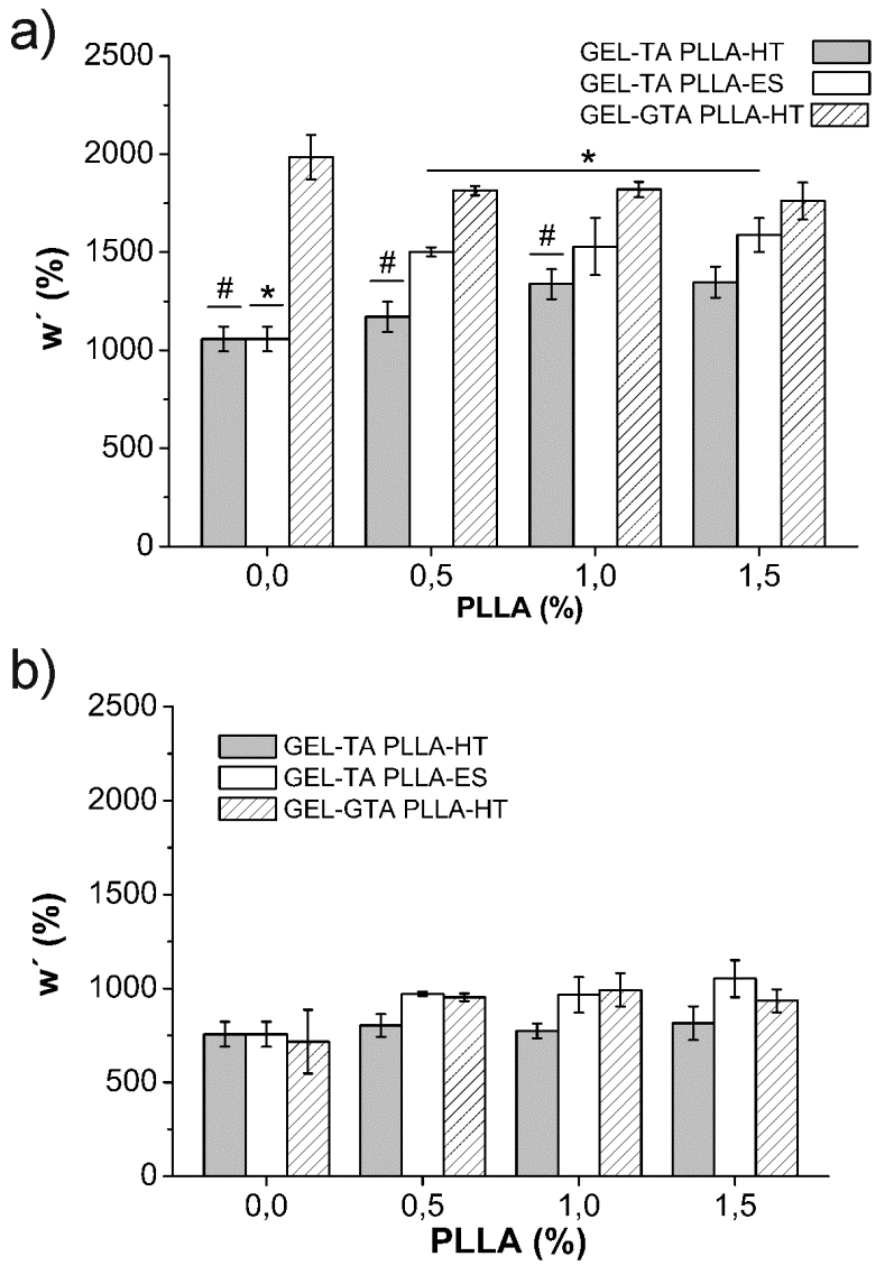

Figure 3-6. Equilibrium water content referred to the dry mass of gelatin for the GEL-TA PLLA-HT, GEL-TA PLLA-ES and GEL-GTA PLLAHT composites: (a) After preparation and (b) after rehydration in $D P B S$. Dash symbols mark the significant differences among the GELTA PLLA-HT composites and the asterisks mark the significant differences in the GEL-TA PLLA-ES composites. 


\subsubsection{Cell culture and seeding}

The formation of the whitish semicircular hydrogel observed in Figure 3-7 was carried out in less than one minute in the pure gelatin (GELTA). However, when the fibers are incorporated in the gelatin/cells solution a varying geometrical shape was obtained after the crosslinking reaction (composite's column in Figure 3-7). The deformed gelatin and the presence of fibers do not show any negative effect on the cell culture. Moreover, after 21 days no hydrogel shrinking was found in comparison with the beginning of the experiment. In fact, we can consider this relatively unusual compared with other bibliographic references where the cells are able to contract the hydrogel molecules. This is the case of collagen hydrogels seeded with $5 \times 10^{4}$ to $5 \times 10^{7}$ cells $/ \mathrm{mL}$ of mesenchymal stem cells (MSCs) [180]-[182] or with $1 \times 10^{6}$ cells $/ \mathrm{mL}$ of porcine smooth muscle cells [165], collagen-methacrylated hyaluronic acid hydrogel seeded with $2 \times 10^{6}$ cells $/ \mathrm{mL}$ of NIH-3T3 cells [183] and fibroblasts seeded in collagen hydrogels [184]-[186]. Figure 3-7 also illustrates the actin cytoskeleton of L929 cells encapsulated in GEL-TA and composites at the culture time of 1 and 21 days. At day 1 , cells are proliferating in the hydrogels, and developed a narrow actin cytoskeleton showing a fairly round shape as typically occurs in cells encapsulated in low stiffness hydrogels for this short period of time [186]-[188]. The cellular growth indicates low toxicity of enzymatically cross-linked hydrogels as reported by previous studies where the compatibility, spreading and cell attachment onto the surface of enzymatically crosslinked GEL-TA was evaluated [129], [189]. After 21 days of culture (Figure 3-7), cells proliferated and achieved confluence, showing a round actin cytoskeleton shape. Moreover, chondrocytes were recently encapsulated in similar substrates with positive results [190], confirming their good performance for use in Tissue Engineering of articular cartilage. 


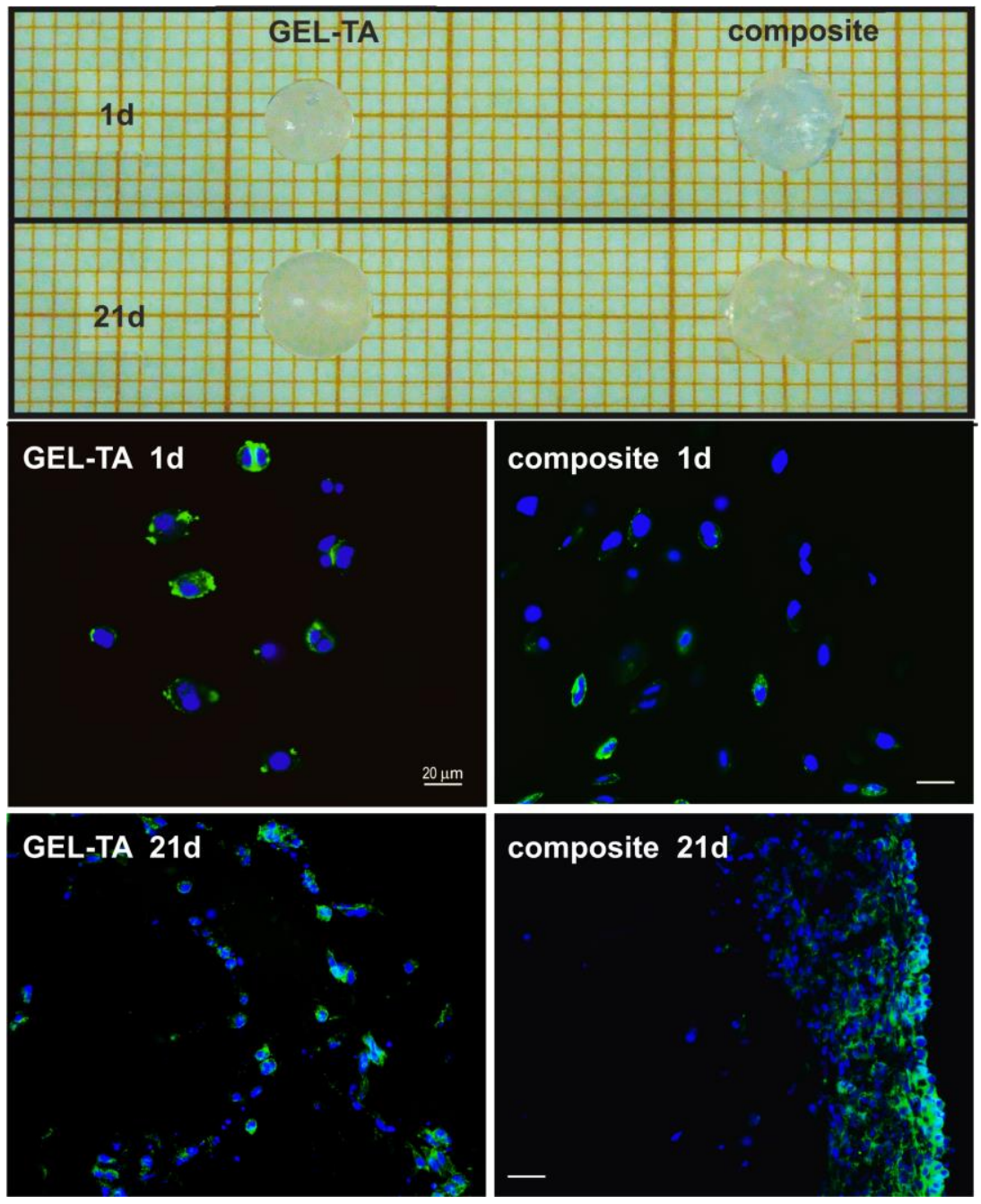

Figure 3-7. Macroscopic view of the hydrogels GEL-TA and composite containing 1\% of PLLA-HT fibers at the beginning and after 21 days of culture. Fluorescence images indicate the cellular cytoskeleton (stained in green) that developed around the nuclei (blue) at 1 and 21 days of culture. 


\subsubsection{Cell viability and distribution}

After the fast cellular encapsulation in enzymatically crosslinked hydrogels no signs of toxicity were identified. Previous cytotoxicity studies in this type of in situ crosslinking gelatin have demonstrated the non-cytotoxicity of the system [129], [189]. Here, the effect of the PLLA-HT fibers in terms of cytotoxicity and cell proliferation in this injectable system is analyzed. The viability of the 1929 cells increased over the incubation time in both hydrogels as indicated in Figure 3-8. In the bar chart it was noticeable that cells are viable since the beginning of the experiment, although after the first day of culture the composite seems to be a better substrate for cell spreading, at least until 14 days of culture when cellular confluence was reached in both substrates. Cell viability was confirmed using fluorescent dyes from 1 to 10 days (see Figure 3-8 bottom). In both hydrogels most of the cells are viable (green dots) and increasing numbers of them were detected with the time of culture by the more abundant green dots seen at 10 days. Conversely some few red dots were stained by EthD-1, indicating very low cell death. But the higher level of red dye seen in the pictures of the composites distinctive to the pure gelatin is due to the PLLA fibers also stained red (see Figure $3-9)$. 

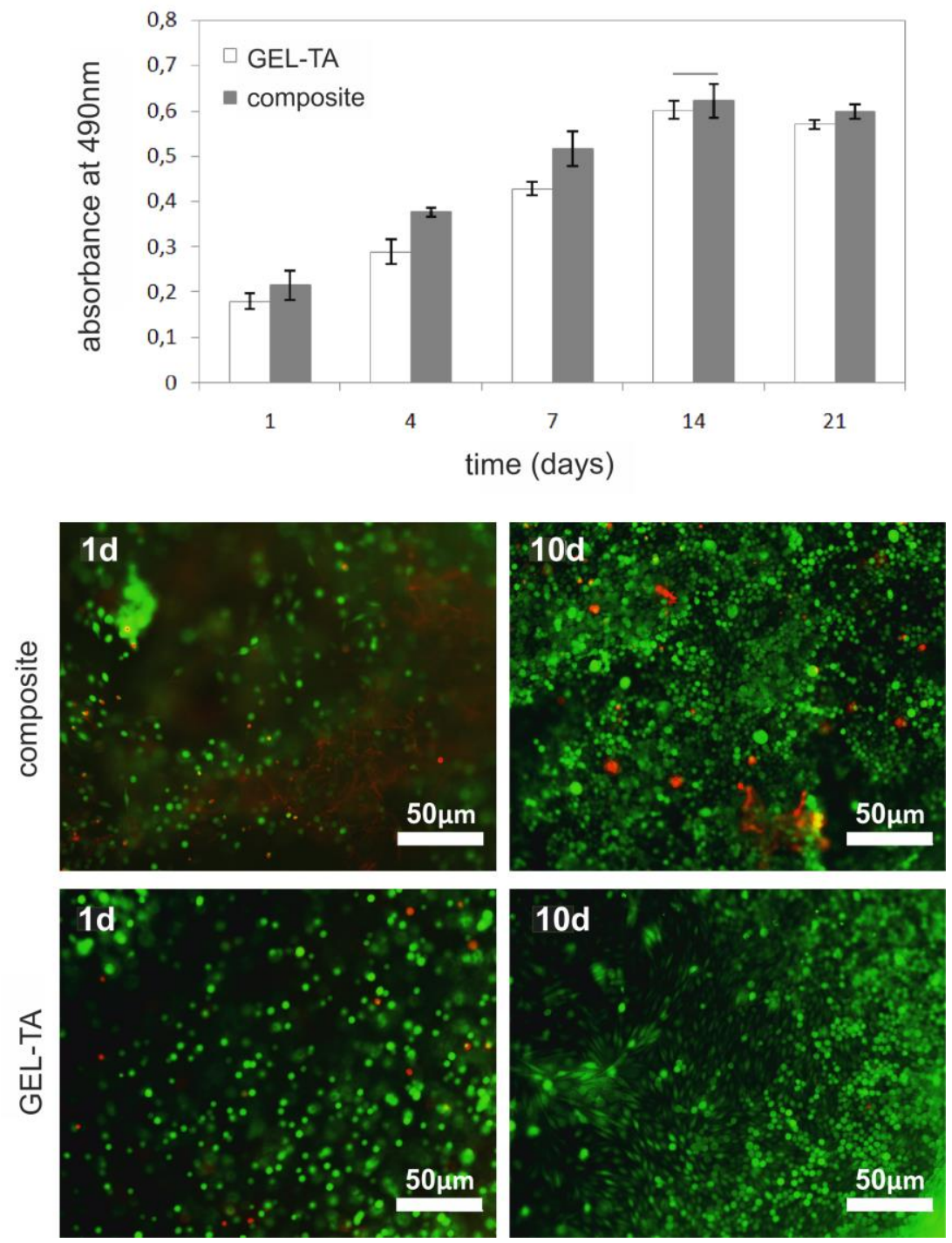

Figure 3-8. Cellular viability measured in GEL-TA and composite containing $1 \%$ of PLLA-HT fibers by MTS (bar graph) and live/dead assays (fluorescent images) for the different culture times. Bar graph shows the average, standard deviation and its level of statistical significance $(p<0.05)$. Line over bars denotes no significant differences. Green staining denotes the viable cells and red staining indicates the dead cells and also the stained PLLA fibers in the composite. 

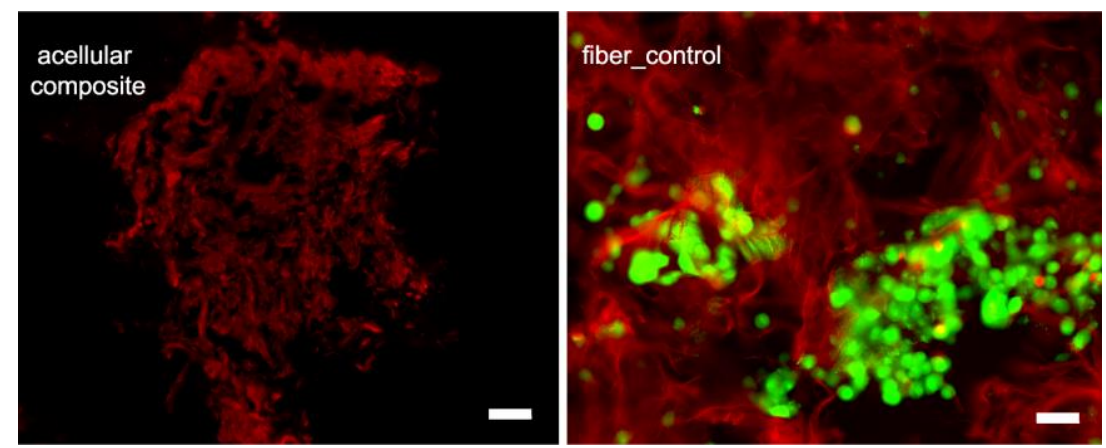

Figure 3-9. Control samples of composites without cells and PLLA fibers with $L 929$ cells cultured in the same conditions were stained with EthD-1 and calcein respectively in order to confirm the fiber dyeing. Scale bar is $20 \mu \mathrm{m}$.

\subsubsection{Conclusions}

Two new families of injectable microfiber-gelatin composites were successfully fabricated by the incorporation and dispersion of new short fibers into the gelatin solution before crosslinking it. A reinforcement of gelatin using PLLA microfibers is obtained in the chemically non-injectable composites (GEL-GTA PLLA-HT) and the injectable ones with the electrospinning milled fibers (GEL-TA PLLAES). However, no reinforcement was observed in the injectable composites with the ultra turbulent obtained fibers (GEL-TA PLLA$\mathrm{HT}$ ). In this last case no significant differences were obtained among the Young's modulus of the samples. The null reinforcement was attributed to the agglomeration of the ultraturbulent obtained fibers in the gelatin solution prior to crosslinking. It could also be due to the weak interaction between the filler and matrix, the formation of a less crosslinked gelatin matrix due to its crosslinking in the presence of fibers and the size and shape of the particles. The gelatin in the composites of the injectable systems has a higher swelling capacity than the pure gelatin, probably due to the presence of gaps between the fibers and the gelatin matrix and/or the lower crosslinking extent of the gelatin, caused by its gelation in the presence of the fibers. The 
injectable matrix is softer than the chemically crosslinked one. All the hydrogels exhibit a microporous structure enabling the flow of nutrients to the cells. The new injectable composite hydrogels are not cytotoxic and induce steady cell growth. 


\subsection{Injectable Gelatin-fiber Composites with Improved Interfacial Interaction}

\subsubsection{Materials and Methods}

\subsubsection{Materials}

Gelatin from porcine skin (gel strength 300 , type A, with $80 \mathrm{mmol}$ $\mathrm{COOH} / 100 \mathrm{~g}$ gelatin), peroxidase from horseradish type I (HRP), potassium phosphate monobasic, 2-hydroxyethyl methacrylate (HEMA) (97\%), sodium phosphate dibasic ( $\geq 99 \%$, ACS Reagent), and benzophenone (ReagentPlus ${ }^{\circledR}$ 99\%) (BP) were provided by SigmaAldrich, Germany. Ethanol ( $96 \%$ vol GPR Rectapur ${ }^{\circledR}$ ) was purchased from VWR International, France. Dimethyl sulfoxide-d6 (D, 99,9\%) and deuterium oxide ( $D, 99,9 \%)$ were provided by Cambridge Isotope Laboratories Inc., MA, USA. Sodium chloride and potassium chloride GR were provided by Merck KGaA, Darmstadt, Germany. Potassium dihydrogen phosphate (analytical reagent, buffer substance) was supplied by Riedel-de Häen $\mathrm{GmbH}$, Seelze, Germany. All the other reagents used in this section and not described here have been detailed in 3.2.1.1. (page 71).

Dulbecco's Phosphate Buffered Saline (DPBS) solution was prepared with $0.2 \mathrm{~g} / \mathrm{L}$ potassium phosphate monobasic, $0.2 \mathrm{~g} / \mathrm{L}$ potassium chloride, $8 \mathrm{~g} / \mathrm{L}$ sodium chloride and $1.15 \mathrm{~g} / \mathrm{L}$ sodium phosphate dibasic.

\subsubsection{PHEMA grafting to loose PLLA microfibers}

PLLA high turbulent flow microfibers were obtained as explained in 3.2.1.2. (page 73) and HEMA was graft polymerized onto PLLA microfibers in a two-step procedure.

Firstly, the PLLA microfibers were activated by dispersing $40 \mathrm{mg}$ fibers in $20 \mathrm{~mL}$ ethanol containing $5 \%(\mathrm{w} / \mathrm{v}) \mathrm{BP}$. This fiber dispersion was placed into a pyrex tube and submitted to an orbital shaking for 5 minutes and then irradiated for 20 min with an UV-lamp (Osram 
Ultra Vitalux, $300 \mathrm{~W}$ ) of $280-320 \mathrm{~nm}$ wavelength and $38 \mathrm{~mW} / \mathrm{cm}^{2}$ output intensity. The dispersion container was covered with a quartz plate to prevent ethanol evaporation and allow penetration of UV light. The distance between the UV lamp and the fiber dispersion was $14 \mathrm{~cm}$. The activated fibers were washed with ethanol two times and then the ethanol was substituted by water drop by drop. Next, 2 $\mathrm{mg} / \mathrm{mL}$ of the activated fibers (PLLA-BP) was dispersed in a $20 \%(\mathrm{v} / \mathrm{v})$ HEMA/ethanol solution and homogenized by orbital shaking for 5 $\mathrm{min}$. The fiber dispersion was then irradiated with UV-light for $30 \mathrm{~min}$ to initiate the PLLA-HEMA grafting and HEMA polymerization, the process took place into a pyrex flask to prevent sample degradation. Unreacted products and non-grafted adsorbed PHEMA chains were removed by washing the fibers twice with ethanol and then the ethanol was substituted by water, injecting it drop by drop to avoid fiber agglomeration.

\subsubsection{Tyramine grafting to gelatin}

Gelatin was modified with tyramine (Gel-Tyr) in order to obtain chains with phenolic groups, which allow hydrogel formation by enzymatic reaction with HRP.

Modified gelatin was obtained by the reaction between its carboxylic groups with amine groups of tyramine [129]. The tyramine molecule (Tyr) was covalently grafted onto gelatin chains by carbodiimide chemistry. The EDC activates the carboxylic groups of gelatin forming an $\mathrm{O}$-acylisourea intermediate. This intermediate is highly reactive and reacts with the tyramine amine group forming a covalent bond. NHS was used as stabilizer of the O-acylisourea group preventing the hydrolytic side reaction [191].

The synthesis procedure has previously been described in [129] with some modifications. The molar ratios for the grafting were tyramine/EDC $/ \mathrm{COOH}=2 / 2 / 1$ and $\mathrm{NHS} / \mathrm{EDC}=1 / 10$.

Briefly, $2 \%(\mathrm{w} / \mathrm{v})$ gelatin in $50 \mathrm{mM}$ MES was dissolved at $60^{\circ} \mathrm{C}$ for 30 min under stirring ( $0.4 \mathrm{~g}$ of gelatin and $0.195 \mathrm{~g}$ of MES in $20 \mathrm{~mL}$ ). 
Then $0.111 \mathrm{~g}$ of tyramine hydrochloride was added and the mixture was stirred for $20 \mathrm{~min}$ at room temperature. The $\mathrm{pH}$ was adjusted to 6 , and $7 \mathrm{mg}$ of NHS was added and stirred for $30 \mathrm{~min}$ for homogenization. Afterwards, $123 \mathrm{mg}$ of EDC was added and the mixture was stirred for another $24 \mathrm{~h}$ at $37^{\circ} \mathrm{C}$. Unreacted reagents were removed via dialysis against deionized water for $48 \mathrm{~h}$. Finally, the modified gelatin was lyophilized in a LyoQuest (Telstar Life Science Solutions, Japan) for further use.

\subsubsection{Enzymatic crosslinking of tyramine grafted gelatin and their PLLA fiber composites}

Two types of composites were prepared: i) gelatin with plasma treated PLLA fibers, and ii) gelatin with PHEMA-grafted PLLA fibers (PLLA-PHEMA).

For the activation of the surface of the PLLA fibers with plasma, which also promotes their wettability, the dried microfibers were submitted to an air plasma treatment ( $300 \mathrm{~W}$, Plasma treatment unit PICCOLO of Plasma Electronic GmbH, Germany) for 5 min.

To obtain the composites of tyramine grafted gelatin (GEL-Tyr) and PLLA fibers (plasma treated or PLLA-PHEMA), different quantities of fibers $(0,0.5$ and $1 \%(w / v))$ were dispersed in $80 \%$ of the total volume of the hydrogel in Krebs Ringer Buffer (KRB). Dispersion was forced by subjecting the mixtures to an ultrasonic treatment ( $225 \mathrm{~W}$, $4 \mathrm{~s}$ on and $2 \mathrm{~s}$ off) for $1 \mathrm{~min}$. After that, $3 \%(\mathrm{w} / \mathrm{v})$ of GEL-Tyr was fully dissolved in the fiber dispersion at $37^{\circ} \mathrm{C}$. Finally, the chemical crosslinking of the GEL-Tyr with or without fibers was achieved using $10 \%$ of the total volume of the hydrogel of a KRB solution of 12.5 $\mathrm{U} / \mathrm{mL}$ of HRP and $10 \%$ of the total volume of the hydrogel of a $20 \mathrm{mM}$ solution of $\mathrm{H}_{2} \mathrm{O}_{2}$ in $\mathrm{KBR}$.

Henceforth, gelatin composites synthesized with plasma treated PLLA fibers are denoted GEL+xPLLAp, while gelatin composites synthesized with PLLA-PHEMA fibers are denoted GEL+xPLLA-PHEMA. In both cases, $x$ indicates the percentage of fibers used in the formation of 
the composite $(0.5 \%$ and $1 \%)$. Enzymatically crosslinked gelatin will be denoted as GEL.

The composites were stored in a fridge in DPBS with $0.02 \%(\mathrm{w} / \mathrm{v})$ sodium azide for further characterization.

\subsubsection{Size Exclusion Chromatography (SEC)}

The average molecular weight and the polydispersity index (PDI) of the PLLA fibers before and after PHEMA grafting were determined with SEC. Fibers were dissolved in chloroform at a concentration of $0.1 \mathrm{mg} / \mathrm{mL}$. Solutions were filtered (Teflon, $0.45 \mu \mathrm{m}$ ), injected with a PL-AS RT autosampler, and analyzed on a Verotech PL-GPC 50 plus system equipped with a PL-RI detector and two PLgel $5 \mu \mathrm{m}$ MIXED-D columns from Varian. Chloroform was used as the mobile phase (1 $\mathrm{mL} / \mathrm{min}, 30^{\circ} \mathrm{C}$ ). The system was calibrated using polystyrene standards with a narrow molecular weight distribution and CirrusTM GPC software was used to process the data.

\subsubsection{Fourier Transformed Infrared (FTIR) Spectroscopy}

Surface grafting was verified by FTIR. Spectra were recorded in the range of $4000-600 \mathrm{~cm}^{-1}$ on a Spectrum 2000 Perkin-Elmer spectrometer equipped with an attenuated total reflectance (ATR) accessory (Golden Gate) that measures to a depth of approximately 1 $\mu \mathrm{m}$ from the sample surface. All FTIR spectra were obtained as the average of 16 individual scans at $1 \mathrm{~cm}^{-1}$ resolution with corrections for atmospheric $\mathrm{H}_{2} \mathrm{O}$ and $\mathrm{CO}_{2}$.

\subsubsection{Proton Nuclear Magnetic Resonance $\left({ }^{1} \mathrm{H}-\mathrm{NMR}\right)$}

${ }^{1} \mathrm{H}$-NMR measurements were performed in $\mathrm{D}_{2} \mathrm{O}$ for the gelatin and the tyramine grafted gelatin and with $\mathrm{CDCl}_{3}$ for the PLLA and PLLAHEMA fibers at a concentration of $10 \mathrm{mg} / \mathrm{mL}$ in a Spectra spin 400 Ultrashield from Bruker. The samples were prepared in NMR sample tubes (5 $\mathrm{mm}$ in diameter) and spectra were evaluated using MestReNova software. 


\subsubsection{Scanning Electron Microscopy (SEM)}

GEL, GEL+1PLLAp and GEL+1PLLA-PHEMA cross-sections were obtained by cutting the lyophilized crosslinked hydrogels with a sharp blade. The samples were examined using a Hitachi S-4800 field emission scanning electron microscope at an accelerating voltage of $1 \mathrm{kV}$. The composites were mounted on adhesive carbon black and sputter-coated with a $10 \mathrm{~nm}$ gold/palladium layer.

\subsubsection{Swelling}

Composites were prepared in cylindrical molds with a diameter of $\sim 8$ $\mathrm{mm}$. After crosslinking, they were immersed in DPBS with $0.02 \%$ sodium azide solution at $37^{\circ} \mathrm{C}$ overnight to reach equilibrium. Then each sample was weighed (this will be the initial water absorption value) in a Mettler AE166 DeltaRange ${ }^{\circledR}$ (Zurich, Switzerland), after rinsing twice with water to remove the DPBS, and finally lyophilized to obtain the dry mass of the sample. Dry samples were immersed again in DPBS with $0.02 \%$ sodium azide at $37^{\circ} \mathrm{C}$ and weighed at different intervals of time to obtain the re-absorption curves of the composites, in terms of the water content, $w_{r}$ (Equation 10):

$$
w_{r}(\%)=\frac{m_{\text {water }}}{m_{\text {dry }}} \cdot 100 .
$$

Moreover, the equilibrium water content $\left(E W C_{r}\right)$ was also obtained as the quantity of water per mass of dry composite when equilibrium was reached and compared with the initial equilibrium water content of the samples $(E W C)$, i.e. before lyophilization.

The water content relative to the quantity of gelatin in the composites in the re-absorption process $\left(w_{r}{ }^{\prime}\right)$ was also calculated as follows, Equation 11:

$$
w_{r}^{\prime}(\%)=\frac{m_{\text {water }}}{m_{\text {gelatin }}} \cdot 100,
$$

which in equilibrium would equal $E W C^{\prime}$ and $E W C_{r}^{\prime}$ for the samples before and after lyophilization, respectively. 


\subsubsection{Rheological measurements}

Rheological experiments were performed on a strain-controlled AR2000ex rheometer (TA Instruments) with the hydrogels immersed in PBS at $25^{\circ} \mathrm{C}$. Cylindrical swollen hydrogels with a diameter of $12 \mathrm{~mm}$ were placed between two parallel plates of nonporous stainless steel. The gap between the plates was adjusted using a normal force of 0.1 $\mathrm{N}$ in order to prevent slippage. A gap higher than $800 \mu \mathrm{m}$ was always reached after sample relaxation until equilibrium. Measurements were always carried out at $25^{\circ} \mathrm{C}$. Sample temperature was controlled and maintained by a Peltier device.

Two different types of measurements in shear deformation mode were performed. First, the range of strain amplitudes for which hydrogels exhibit a linear region of viscoelasticity was determined. A dynamic strain sweep (with amplitudes ranging between $0.01 \%$ and $20 \%$ ) was carried out at the frequency of $1 \mathrm{~Hz}$ to measure the dynamic shear modulus as a function of the strain. Second, dynamic frequency sweep tests were performed to measure the dependence of the dynamic shear modulus and the loss factor on frequency. Specifically, a frequency sweep between $0.01 \mathrm{~Hz}$ and $10 \mathrm{~Hz}$ at a fixed strain (corresponding to the hydrogel linear region) was selected.

The storage modulus $\left(G^{\prime}\right)$, the loss modulus $\left(G^{\prime \prime}\right)$, the complex modulus magnitude $\left(\left|G^{*}\right|\right.$, a measure of the hydrogel stiffness: $\left|G^{*}\right|^{2}$ $\left.=\left(G^{\prime}\right)^{2}+\left(G^{\prime \prime}\right)^{2}\right)$, and the loss factor $\left(\tan \delta=\left(G^{\prime \prime}\right) /\left(G^{\prime}\right)\right.$, a measure of the internal energy dissipation, $\delta$ being the phase angle between the applied stimulus and the corresponding response) as a function of the strain amplitude or the frequency were obtained. 


\subsubsection{Cell culture}

Mouse fibroblasts 1929 were expanded in $75 \mathrm{~cm}^{2}$ flasks and incubated in a $5 \% \mathrm{CO}_{2}$ humidified atmosphere at $37{ }^{\circ} \mathrm{C}$ until confluence. DMEM with $4.5 \mathrm{~g} / \mathrm{L}$ glucose supplemented with $10 \% \mathrm{FBS}$, $1 \%$ penicillin/streptomycin (100 U/mL / $100 \mathrm{mg} / \mathrm{mL})$ and $1 \% \mathrm{~L}$ glutamine, was used as culture media. After confluence, cells were harvested, counted with a hemocytometer and encapsulated in the GEL hydrogel (prepared at 3\% (w/v)) and in the composite with $1 \%$ of PLLA-PHEMA fibers.

Each hydrogel was prepared with $80 \%(\mathrm{v} / \mathrm{v})$ of $3 \%(\mathrm{w} / \mathrm{v})$ GEL-Tyr, $10 \%$ $(\mathrm{v} / \mathrm{v})$ of $\mathrm{HRP}$ at $12.5 \mathrm{U} / \mathrm{ml}$ (that is $1.25 \mathrm{U} / \mathrm{ml}$ in the final volume) and $10 \%(\mathrm{v} / \mathrm{v})$ of $20 \mathrm{mM} \mathrm{H}_{2} \mathrm{O}_{2}$ (2 $\mathrm{mM}$ in the final volume). The GEL-Tyr and HRP were mixed and sterilized with a $0.22 \mu \mathrm{m}$ filter. Fibroblasts were included in the GEL-Tyr-HRP solution at a concentration of $2 \times 10^{5}$ cells $/ \mathrm{mL}$. The samples were formed by mixing $45 \mu \mathrm{L}$ of the cell GELTyr-HRP solution and $5 \mu \mathrm{L}$ of $\mathrm{H}_{2} \mathrm{O}_{2}(20 \mathrm{mM})$ in a 48-well plate. After $15 \mathrm{~min}, 1 \mathrm{~mL}$ of medium was added to each well and the plate was then incubated for $1,7,14$ and 21 days. The medium was renewed every 3 days.

\subsubsection{Cell viability (MTS)}

Cell viability at all times of culture was confirmed using the MTS assay (3-(4,5-dimethylthiazol-2-yl)-5-(3-carboxymethoxyphenyl)-2-(4-

sulfophenyl)-2H-tetrazolium) following manufacturer instructions. Briefly, the cell cultured samples were washed twice with DPBS and incubated with fresh culture medium without neither phenol red nor FBS but containing MTS reagent (ratio $5: 1$ ) at $37^{\circ} \mathrm{C}$ for $3 \mathrm{~h}$ in darkness. Thereafter, the absorbance was measured at $490 \mathrm{~nm}$ with a Victor 3 microplate reader (Perkin-Elmer, Waltham, MA, U.S.A.). 


\subsubsection{Cell distribution and shape by nuclei and cytoskeleton staining}

To examine cell shape and distribution within the hydrogels, cell nuclei and cytoskeleton were processed for fluorescence microscopy observation after 1, 7 and 14 days of culture.

Prior to staining, cultured samples were washed twice with DPBS, and then fixed with $10 \%$ formalin at $4^{\circ} \mathrm{C}$ for 20 min. After fixing, the samples were washed twice in DPBS and incubated for $10 \mathrm{~min}$ with $0.5 \%$ Triton X-100 at room temperature. After cell membrane permeation with Triton, samples were washed twice with DPBS and actin cytoskeleton was stained by $1 \mathrm{~h}$ incubation in 1:40 BODIPY FL Phallacidin. Then, samples were washed with DPBS and left overnight into a $30 \% \mathrm{w} / \mathrm{v}$ sucrose solution, followed by their inclusion in OCT at $-80^{\circ} \mathrm{C}$ to obtain $50 \mu \mathrm{m}$ sections in a Leica $\mathrm{CM} 1520$ cryotome at $-20^{\circ} \mathrm{C}$. Cellular nuclei were labeled by DAPI prior to the observation of the sections under the microscope. Photomicrographs were taken using the fluorescent microscope Eclipse 80i.

\subsubsection{Results and discussion}

A new injectable in situ forming gelatin-fiber composite with enhanced mechanical properties was here achieved through the reinforcement of tyramine-gelatin hydrogels with PHEMA-surface grafted PLLA microfibers.

\subsubsection{PHEMA grafting on PLLA microfibers}

The surface modification of the PLLA micro-fibers was mediated by a sequential photoinduced graft polymerization method [192]-[195]. Firstly, the PLLA fibers were dispersed in an ethanol bath containing the benzophenone initiator which abstracts hydrogens from the PLLA substrate, creating radicals on the surface of the fibers and semipinacol radicals, which combine to form surface photoinitiators (Figure 3-10). Next, these surface photoinitiators are dispersed in an ethanol solution containing HEMA and initiate graft polymerization 
under UV irradiation. UV irradiation converts the surface initiators to surface radicals and semipinacol radicals. HEMA monomers react preferably with these surface radicals than with the semipinacol radicals due to steric reasons, resulting in oligomeric and polymeric PHEMA chains grafted onto the PLLA fibers (Figure 3-10).

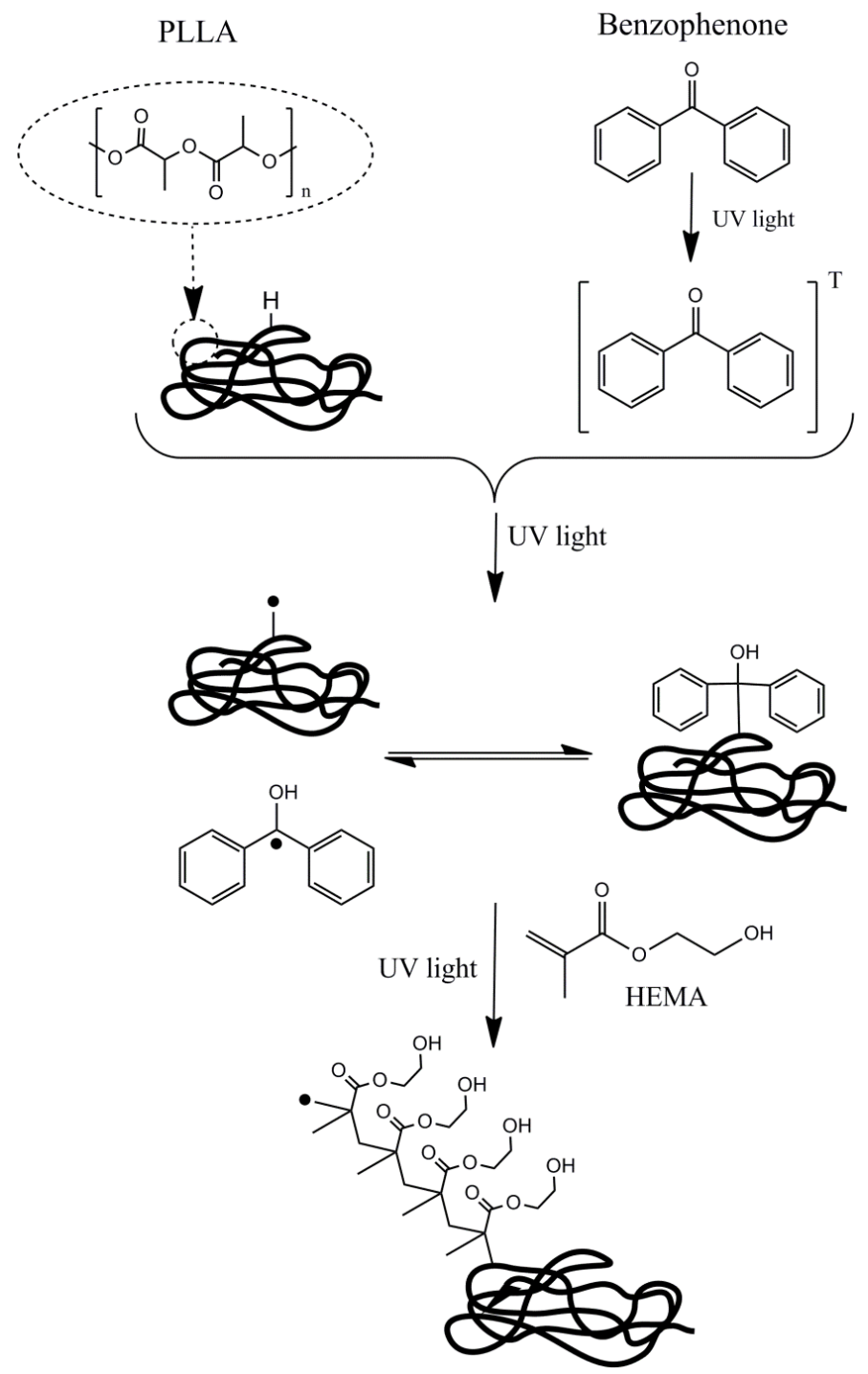

Figure 3-10. Scheme of the chemical reaction produced to graft the PHEMA chains on the PLLA microfibers. 
Not a high reduction in the molecular weight of the PLLA is expected in this reaction, as the only effect of benzophenone is the abstraction of hydrogen bonds from superficial chains of the fibers and the subsequent combination of generated radicals with HEMA monomer. In fact, previous reported results of this grafting process on PLA particles named this synthesis "nondestructive covalent graftingfrom", as it is applicable to complex geometries while maintaining the shape of the substrate without degradation [196]. The mean number average molecular weight (Mn) of PLLA fibers resulted $197000 \mathrm{~g} / \mathrm{mol}$ with a polydispersity index (PDI) of 1.44, which are consistent with the data provided by the supplier. UV irradiation [192], [195] and water washings produced a small reduction in the molecular weight of the PLLA-PHEMA fibers which had a mean $\mathrm{Mn}$ value of $153000 \mathrm{~g} / \mathrm{mol}$ with a PDI of 1.21 . It should be noted that the solubility of PLLA-PHEMA in chloroform is significantly reduced compared to the ungrafted PLLA fibers, which will influence the hydrodynamic volume and make comparisons between the molecular weight values as measured by SEC more difficult.

FTIR and ${ }^{1} \mathrm{H}-\mathrm{NMR}$ analysis verify grafting of PLLA microfibers with PHEMA. FTIR spectra of PLLA microfibers, PLLA-BP activated microfibers and PLLA-PHEMA grafted microfibers (Figure 3-11) show the appearance of a broad band at $3500 \mathrm{~cm}^{-1}$ (peak \#1, Figure 3-11) on the PLLA-PHEMA surface arising from the additional -OH groups in the PHEMA chains grafted onto the fibers [197]. Overlaid $C=0$ stretching bands at $1750 \mathrm{~cm}^{-1}$ (peak \#2, Figure 3-11) show the presence of two different types of carbonyl groups, stemming from PLLA main chain ester bonds and PHEMA repeating units, respectively, which is another indication of the successful grafting to the microfibers. It is worth mention that PHEMA homopolymerization could take place under UV radiation without the need of the PLLA-BP surface initiatior [194], [196]. However, those PHEMA hompolymer chains that were not grafted to the PLLA fibers should not remain adsorbed on the PLLA fibers as they are soluble in ethanol and water, the solvents we used after the synthesis to wash 
off unreacted products and non-grafted PHEMA chains. The surface activated PLLA fibers give rise to bands at $3500 \mathrm{~cm}^{-1}$ (-OH, peak \#3, Figure 3-11), $1750 \mathrm{~cm}^{-1}$, and $1250 \mathrm{~cm}^{-1}$ (aromatic rings, peak \#4, Figure 3-11) which are all characteristic of BP.

Figure 3-12 corresponds to the ${ }^{1} \mathrm{H}-\mathrm{NMR}$ spectrum of the PLLA-PHEMA microfibers in comparison to the PLLA ones. Two hydrogens of the monomeric units of the PLLA can be observed at $5.2 \mathrm{ppm}$ and 1.56 ppm [198] (Figure 3-12b). These peaks are still seen in the ${ }^{1} \mathrm{H}-\mathrm{NMR}$ spectrum of the PLLA-PHEMA grafted microfibers (Figure 3-12a). The other peaks observed in Figure 3-12a at $1.26-0.59 \mathrm{ppm}$ are due to the PHEMA hydrogen protons $\left(\mathrm{CH}_{2}-\mathrm{C}\left(\mathrm{CH}_{3}\right)\right)$ [199], indicating effective grafting. Although the covalent linkage between PLLA and PHEMA does not have any hydrogen that could give a specific signal in the ${ }^{1} \mathrm{H}$ NMR spectra, we can say that the peaks of PHEMA correspond to grafted PHEMA, as eventual adsorbed PHEMA should be removed in the ethanol and water baths used to wash the fibers [196]. Moreover, PLLA is soluble in chloroform (the solvent we used to perform ${ }^{1} \mathrm{H}$-NMR spectra) but the homopolymer PHEMA is not. If the PLLA-PHEMA was a physical blend rather than a graft-copolymer, dissolution would result in a soluble PLLA fraction and an insoluble fraction, and a pure PLLA spectrum would show upon NMR analysis. The ${ }^{1} \mathrm{H}$-NMR spectrum in Figure $3-12 \mathrm{a}$ however, shows peaks originating from both PLLA chain segments and HEMA graft chains indicating that the PLLA and HEMA segments are indeed covalently linked. 


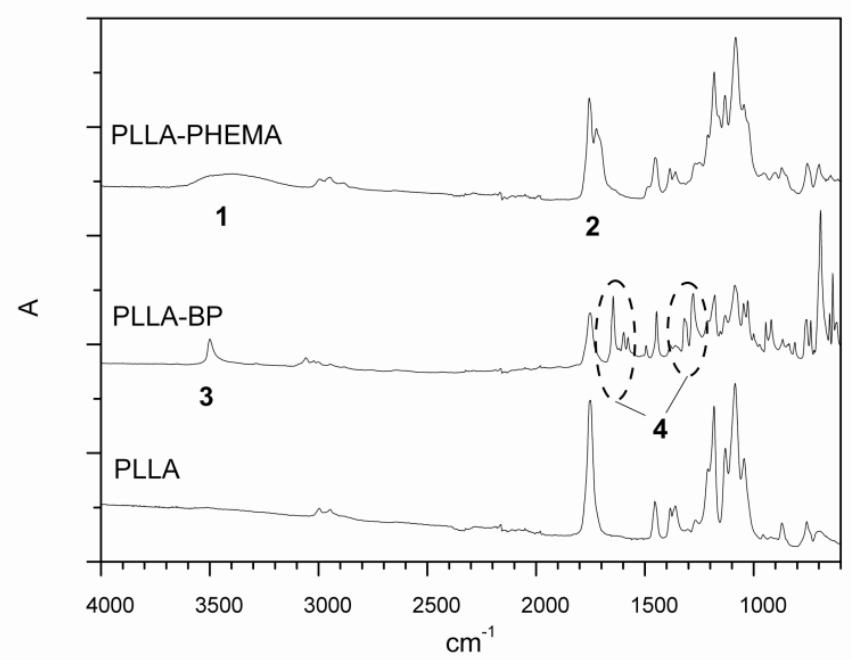

Figure 3-11. FTIR spectra of (from bottom to top) PLLA microfibers, benzophenone activated fibers (PLLA-BP), and PLLA-PHEMA grafted microfibers.

a) PLLA-PHEMA

b) PLLA

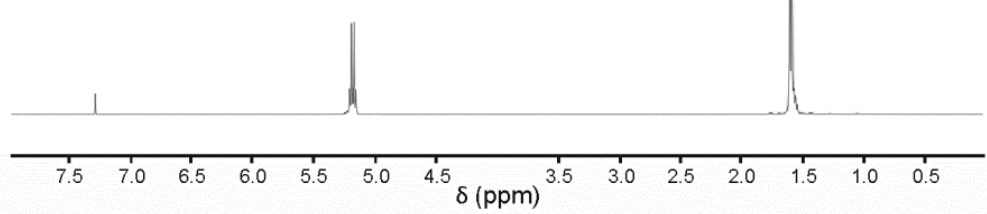

Figure 3-12. ${ }^{1}$ H-NMR of a) PLLA-PHEMA and b) PLLA microfibers. 


\subsubsection{Tyramine grafting on gelatin}

Effective grafting of the tyramine molecules on the gelatin chains was also verified by ${ }^{1} \mathrm{H}-\mathrm{NMR}$. Figure $3-13$ shows the spectra of non modified gelatin (Figure 3-13a) and the tyramine modified gelatin (GEL-Tyr) (Figure 3-13b), where the presence of distinctive peaks for both pairs of aromatic ring protons at 7.0 and $6.7 \mathrm{ppm}$ in the GEL-Tyr spectrum is an indication of successful grafting [106], [200]. The result was also confirmed by UV spectrophotometry which provided a value of $1.9 \cdot 10^{-7}$ tyramine grafted groups per $\mathrm{mg}$ of gelatin by relating the UV absorbance at $275 \mathrm{~nm}$ of the GEL-Tyr sample [129] (results not shown) to a tyramine calibration curve.

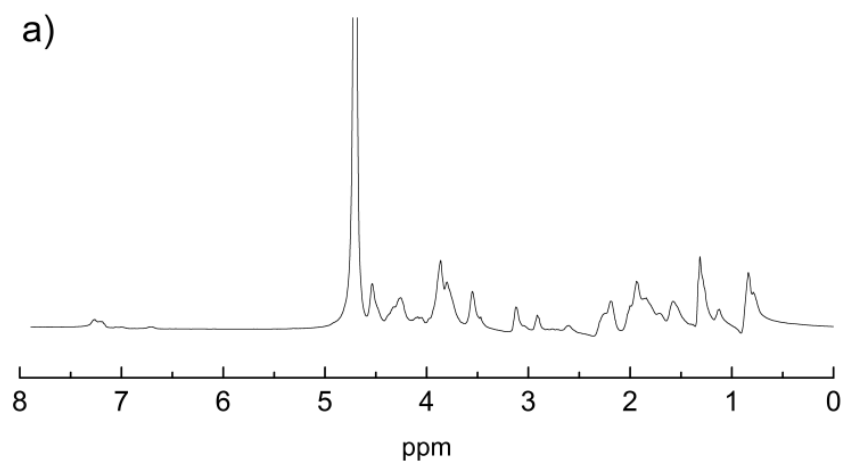

b)

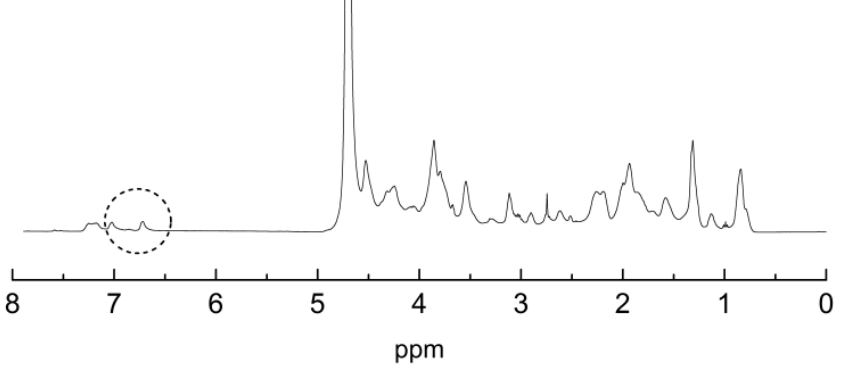

Figure $3-13 .{ }^{1} \mathrm{H}-\mathrm{NMR}$ of a) gelatin (control) and b) tyramine grafted gelatin (GEL-Tyr). 


\subsubsection{Morphology of the composites}

Injectable composite hydrogels were successfully prepared. The HRP is the reaction catalyst and the $\mathrm{H}_{2} \mathrm{O}_{2}$ acts as the oxidation agent, obtaining two water molecules per two oxidized tyramine molecules (phenol groups) [201] in the GEL-Tyr conjugates. Afterwards, as in the injectable gelatin hydrogels prepared in the previous section, the oxidized phenols crosslink through a C-C linkage between the orthocarbons of the aromatic ring or a C-O linkage between the orthocarbon and the phenolic oxygen [136], [200], [202], see Figure 1-10. The macroscopic aspect of the resulting hydrogels, with and without loose PLLA fibers, can be seen in Figure 3-14. Hydrogels with PLLA fibers can be distinguished by a more whitish color than the pure gelatin gel. Nevertheless, no differences in color or homogeneity between the two types of fiber composites are macroscopically detectable.

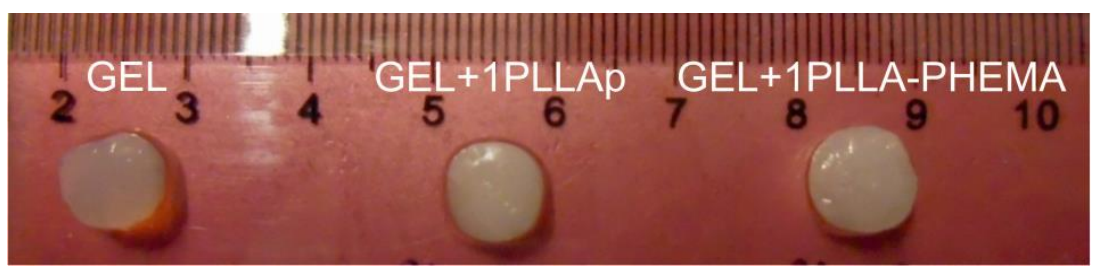

Figure 3-14. Macroscopic image at $25^{\circ} \mathrm{C}$ of the water swollen hydrogels: GEL, GEL+1PLLAp and GEL+1PLLA-PHEMA.
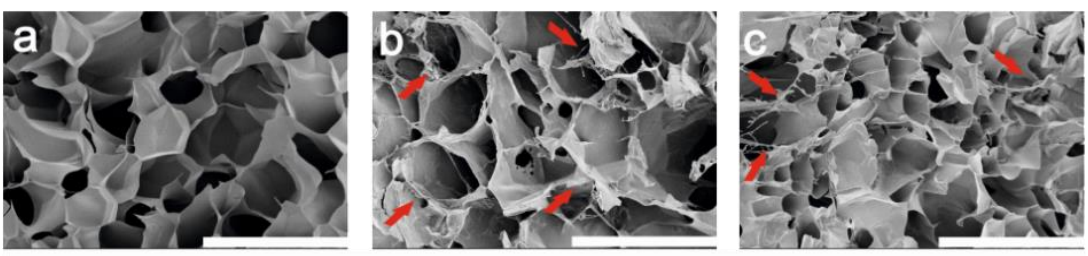

Figure 3-15. SEM images of (a) GEL, (b) GEL+1PLLAp and (c) GEL+1PLLA-PHEMA cross-sections at $1 \mathrm{kV}$. Red arrows mark the PLLA fibers. White scale bar in all pictures corresponds to $50 \mu \mathrm{m}$. 
The internal morphology of the hydrogels with and without loose PLLA fibers was visualized with SEM (Figure 3-15).

Figure 3-15a shows the microstructure of pure gelatin-tyramine crosslinked hydrogel. GEL exhibits a clear and regular porous structure, with pores of about $20 \mu \mathrm{m}$, which allow the water and nutrient diffusion needed for the cell survival and tissue regeneration. When PLLA microfibers are embedded in the gelatin matrix a more heterogeneous matrix is obtained with fibers well distributed in the hydrogel (red arrows in Figure 3-15b and Figure 315c). Hydrogels containing PLLA fibers have a seemingly wider pore size distribution, where bigger pores than in pure gelatin are combined with very small pores, giving rise to a less regular structure.

\subsubsection{Swelling}

The re-absorption curves for GEL+PLLAp and GEL+PLLA-PHEMA composites can be seen in Figure $3-16 a$ and Figure $3-16 b$, respectively. Re-absorption is quite rapid and it seems that the PLLA fibers in both series accelerate the re-absorption process, as equilibrium is reached within $2 \mathrm{~h}$ for the composites, while a longer time is required for pure gelatin. This can be attributed to the fact that the fibers to some extent prevent gelatin pores from collapsing when drying, facilitating water diffusion through the gelatin matrix and giving rise to higher recuperation rates.

Comparing the equilibrium water content in the re-absorption process $\left(E W C_{r}\right)$ to the initial amount of water at equilibrium (EWC), i.e. before subjecting the samples to lyophilization (see Table 3-2), a decrease of more than half the initial value is observed in the reabsorption. This phenomenon has been previously attributed to the pores collapsing during lyophilization, thereby reducing gelatin chain reorganization in the re-absorption process [203]. A similar reduction is also seen when the amount of water is calculated relative to the mass of gelatin in the composites (EWC' and $E W C_{r}^{\prime}$ in Table 3-2). 
a)

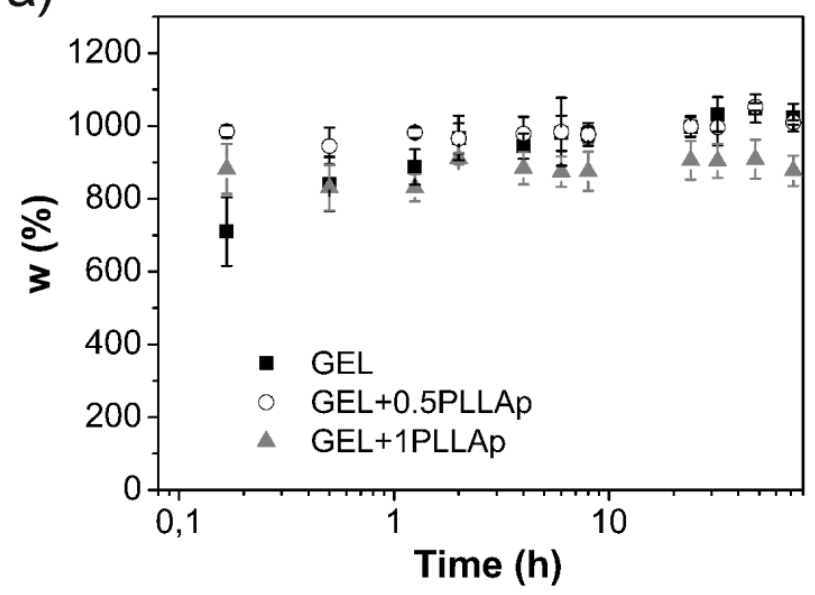

b)

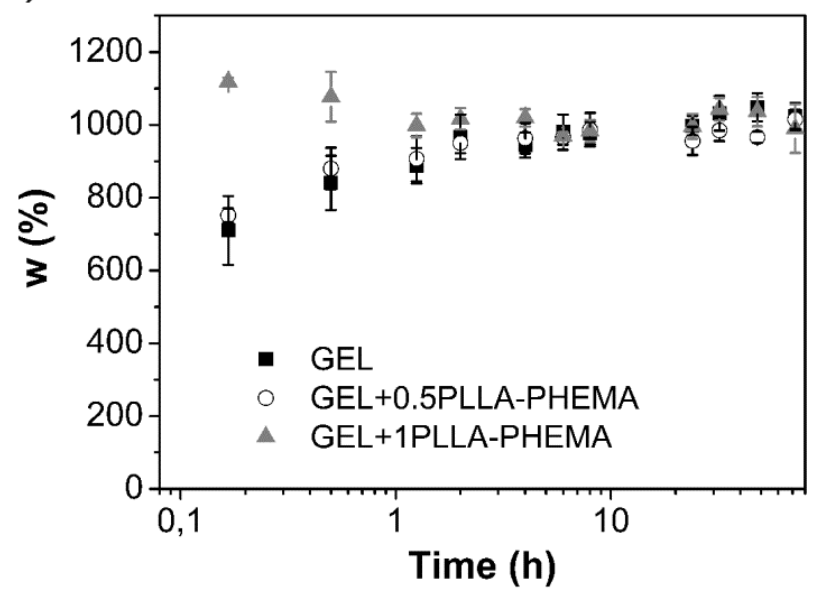

Figure 3-16. Re-absorption curves of (a) GEL+PLLAp composites and (b) GEL+PLLA-PHEMA composites at $37^{\circ} \mathrm{C}$ in DPBS with $0.02 \%$ sodium azide. 
Table 3-2. Equilibrium water contents for the different GEL+PLLA composites before lyophilization relative to sample weight (EWC) or to the weight of gelatin in the samples $\left(E W C^{\prime}\right)$, and in the reabsorption relative to sample weight $\left(E W C_{r}\right)$ and the weight of gelatin in the samples $\left(E W C_{r}^{\prime}\right)$.

\begin{tabular}{lcccc}
\hline Sample & EWC (\%) & EWC $_{\mathbf{r}}$ (\%) & EWC'(\%) $^{\prime}$ & EWC $_{r}^{\prime}(\%)$ \\
\hline GEL & $2439 \pm 61$ & $1023 \pm 38$ & $2439 \pm 61$ & $1023 \pm 38$ \\
GEL+0.5 PLLAp & $2369 \pm 204$ & $1010 \pm 6$ & $2862 \pm 247$ & $1220 \pm 8$ \\
GEL+1 PLLAp & $1860 \pm 41$ & $877 \pm 41$ & $2635 \pm 58$ & $1242 \pm 59$ \\
GEL+0.5 & $2290 \pm 52$ & $1015 \pm 27$ & $2767 \pm 62$ & $1227 \pm 33$ \\
PLLA-PHEMA & & & & \\
GEL+1 & $2036 \pm 48$ & $990 \pm 67$ & $3060 \pm 68$ & $1402 \pm 94$ \\
PLLA-PHEMA & & & & \\
\hline
\end{tabular}

The amount of absorbed water per mass of gelatin (EWC') was calculated in order to see the effect of the fibers in the water sorption of gelatin. Considering that PLLA is a hydrophobic polymer and its water sorption capacity is very low in comparison to gelatin, values of EWC' for all the composites should be the same as for pure gelatin and independent of the fiber content in the composites. However for the PLLA plasma fiber composites, EWC' values are slightly higher than those for pure gelatin, although no differences are obtained between the two composites ( $0.5 \%$ or $1 \%$ of fibers) (Table 3-2). This difference could be attributed to the effect of the air plasma treatment creating hydrophilic groups (hydroxyl-, carboxyl- or amino-) on the fiber surface. In the case of the PLLA-PHEMA composites, the observed increase in the water sorption capacity relative to the weight of gelatin is explained by the PHEMA hydrophilic chains grafted on the fibers' surface, which is another proof of the effectiveness of the chemical modification. These PHEMA chains in the fibers provide one hydroxyl group per 
monomeric unit, improving the fibers' hydrophilicity and thus their interaction with the hydrogel gelatin matrix.

\subsubsection{Rheological properties}

Hydrogels have to be tested within their respective linear viscoelastic range to ensure their stability [204]-[206]. $\left|G^{*}\right|$ remains independent of the strain amplitude (linear viscoelastic behavior), at least, up to about $6-7 \%$, (Figure 3-17) for every measured gel. Thus, in order to ensure that measurements are done within the linear viscoelastic region, all subsequent rheological tests were performed at $1 \%$ strain.

As can be seen in Figure 3-17, microfiber-gelatin composites prepared with the PHEMA grafted fibers have a consistently higher complex modulus magnitude than pure gelatin, indicating an improvement in the interaction between the filler and the matrix. The increase of $\left|G^{*}\right|$ is proportional to the percentage of fibers in the composites. Specifically, a ratio of $\sim 2$ is obtained, when the percentage of fibers is doubled in PLLA-PHEMA containing hydrogels. On the contrary, no significant differences are found for the composites prepared with the ungrafted fibers (PLLAp) when the percentage of fibers is changed. 


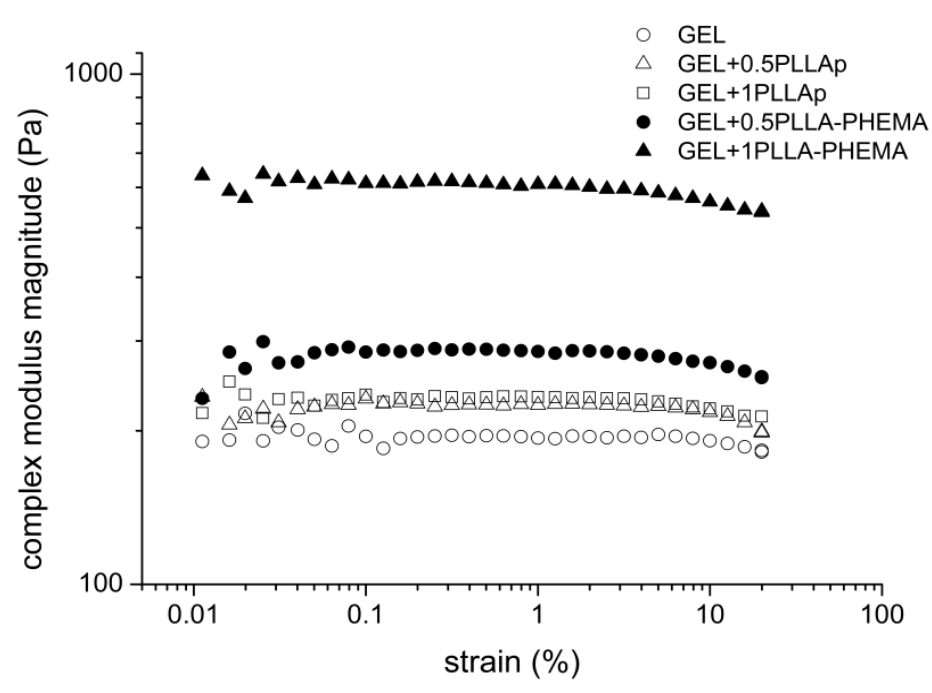

Figure 3-17. Dependence of the complex modulus magnitude $\left(\left|G^{*}\right|\right)$ on the strain amplitude for the fiber-reinforced gelatin gels with (PLLA-PHEMA) and without (PLLAp) hydrophilic grafting at a frequency of $1 \mathrm{~Hz}$ and $25^{\circ} \mathrm{C}$. The results obtained for the pure gelatin gel (GEL, control sample) have been included for comparison. Each curve corresponds to the average of three different samples measured.

In Figure 3-18 the dependence of $G^{\prime}, G^{\prime \prime}$ and $\tan \delta$ on the frequency are shown for grafted (PLLA-PHEMA) and ungrafted (PLLAP) fiberreinforced gelatin gels. $G^{\prime}$ and $G^{\prime \prime}$ increase with frequency for both types of gels, but this trend is only noticeable for the PHEMA grafted samples. As far as the loss factor is concerned, no significant dependence on the frequency is found within the frequency range analyzed. 
a)

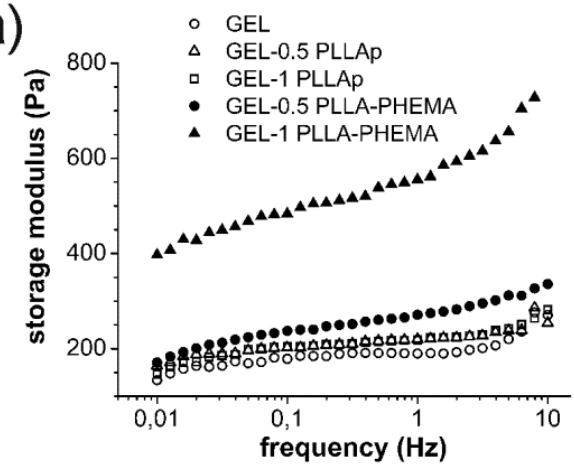

b)

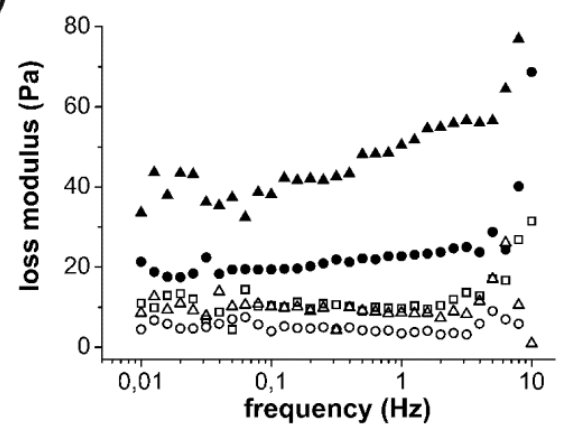

c)

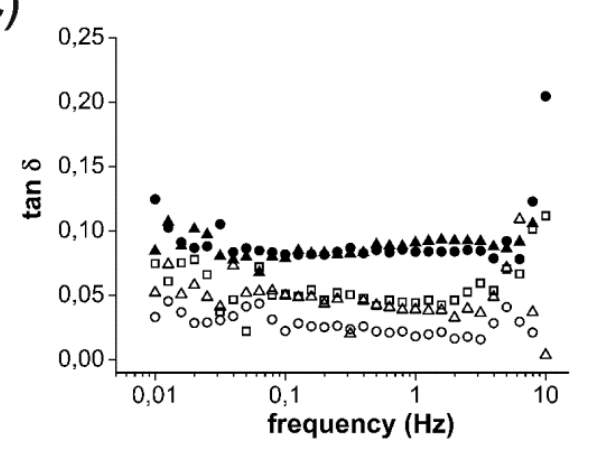

Figure 3-18. Evolution of (a) the storage $\left(G^{\prime}\right)$, and (b) loss $\left(G^{\prime \prime}\right)$ moduli, and (c) the loss factor (tan $\delta$ ) as a function of the frequency for the fiber-reinforced gelatin gels with (PLLA-PHEMA) and without (PLLAp) hydrophilic grafting at $25^{\circ} \mathrm{C}$. The results obtained for the unreinforced gelatin gel (GEL, control sample) have been included for comparison. Each curve corresponds to the average of three individual samples measured. The legend seen in (a) is valid for all the graphs. 
Focusing on a fixed frequency, $\mathrm{G}^{\prime}, \mathrm{G}^{\prime \prime}$ and $\tan \delta$ values at $1 \mathrm{~Hz}$ have been included in Table 3-3 for all the samples.

The storage modulus is the dominant contribution to $\left|G^{*}\right|$, since $G^{\prime}$ $>G^{\prime \prime}$ in both types of gels, verifying that the gels indeed display an elastic behavior. The value of $\mathrm{G}^{\prime}$ at $1 \mathrm{~Hz}$ appears in the range of several hundreds of $\mathrm{Pa}$, and specifically, for $1 \%$ PLLA-PHEMA is higher than $500 \mathrm{~Pa}$.

Estimated loss factors are $2.5^{\circ}$ and $5^{\circ}$ for gels containing ungrafted and grafted-PLLA fibers, respectively, regardless of the fiber percentage. Taking into account that $\tan \delta$ measures the internal energy dissipation, the interphase interactions occurring in the PLLAPHEMA grafted gels may give rise to an increase in the phase angle (around a 2-fold change) with respect to ungrafted hydrogels. Still, both angles are very low, characteristic of highly elastic, energy storing hydrogels.

Table 3-3. Storage $\left(G^{\prime}\right)$, and loss $\left(G^{\prime \prime}\right)$ moduli, loss factor (tan $\delta$ ), and phase angle $(\delta)$ evaluated at a frequency of $1 \mathrm{~Hz}$, using an $1 \%$ strain and at $25^{\circ} \mathrm{C}$ for the fiber-reinforced gelatin gels with (PLLA-PHEMA) and without (PLLAp) hydrophilic grafting. The results obtained for the unreinforced gelatin gel (GEL, control sample) have been included for comparison.

\begin{tabular}{lcccr}
\hline Sample & $\mathbf{G}^{\prime}(\mathrm{Pa})$ & $\mathbf{G}^{\prime \prime}(\mathrm{Pa})$ & $\tan \boldsymbol{\delta}$ & $\boldsymbol{\delta}\left(^{\circ}\right)$ \\
\hline GEL & $184.9 \pm 32$ & $3.4 \pm 0.4$ & $0.019 \pm 2 \times 10^{-3}$ & $\sim 1.1$ \\
GEL+0.5 PLLAp & $219.8 \pm 15$ & $8.6 \pm 1.6$ & $0.038 \pm 4 \times 10^{-3}$ & $\sim 2.2$ \\
GEL+1 PLLAp & $218.6 \pm 14$ & $9.7 \pm 0.4$ & $0.048 \pm 4 \times 10^{-3}$ & $\sim 2.5$ \\
GEL+0.5 & $271.2 \pm 47$ & $22.7 \pm 3.1$ & $0.084 \pm 6 \times 10^{-3}$ & $\sim 4.8$ \\
PLLA-PHEMA & & & & \\
GEL+1 & $535.2 \pm 90$ & $48.5 \pm 7.0$ & $0.091 \pm 7 \times 10^{-3}$ & $\sim 5.2$ \\
PLLA-PHEMA & & & & \\
\hline
\end{tabular}


The degree of interaction between the short fibers and the matrix of our reinforced hydrogels determines their mechanical properties [162]. According to the rheological results, the inclusion of a hydrophilic graft in the PLLA fibers improves the interaction between the fibers and the gelatin matrix. The complex fiber/matrix network shown in Figure 3-15b and Figure 3-15c gives rise to an enhancement of the load transmission from the matrix to the fibers and, in consequence, the mechanical integrity of the hydrogels is enhanced.

The interaction between the gelatin matrix and the two types of PLLA fibers is schematically presented in Figure 3-19. The higher amount of hydroxyl groups on the PLLA-PHEMA microfibers increases the quantity of hydrogen bonds and therefore improves the interaction with the hydrophilic gelatin matrix, which presents carboxylic and amine groups.

๑ PLLA fiber

- Gelatin

- Hydrogen bonds

он-fон PHEMA chains

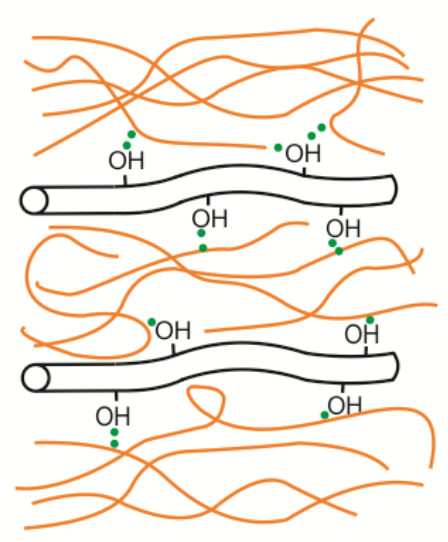

Plasma treated fibers

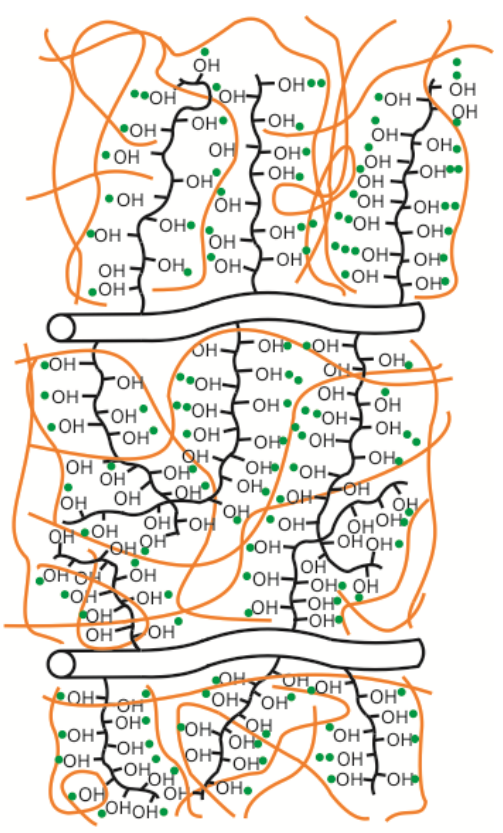

PHEMA grafted fibers

Figure 3-19. Gelatin-fiber interaction scheme for plasma treated PLLA fibers and PLLA-PHEMA fibers. 


\subsubsection{Cytotoxicity of the composites}

After the fast cellular encapsulation in enzymatically crosslinked hydrogels no signs of toxicity were identified. Previous cytotoxicity studies in this type of in situ crosslinking gelatin have demonstrated the non-cytotoxicity of the system [129], [189]. Our purpose was to analyze the non-cytotoxic effect of the PLLA-PHEMA grafted fibers in view of the application of these new injectable composites in tissue engineering. The viability of the $L 929$ cells increased until the $7^{\text {th }}$ day of culture and the 14th day of culture for the GEL and GEL+1PLLAPHEMA hydrogels, respectively, as indicated in Figure 3-20. At this time, cells stop growing by the presence of gelatin chains or fibers and no more proliferation is observed. Interestingly, the proliferation of cells for short times ( 1 and 7 days) is lower in the composite with PLLA-PHEMA fibers. This lower proliferation rate could be due to the difficulties that cells find in moving the fibers in order to grow. This is easier when the matrix consists of gelatin only, and is probably also due to the presence of PHEMA chains hindering cell attachment [207], [208]. Thus, in the case of the composite, proliferation stops at 14 days of culture.

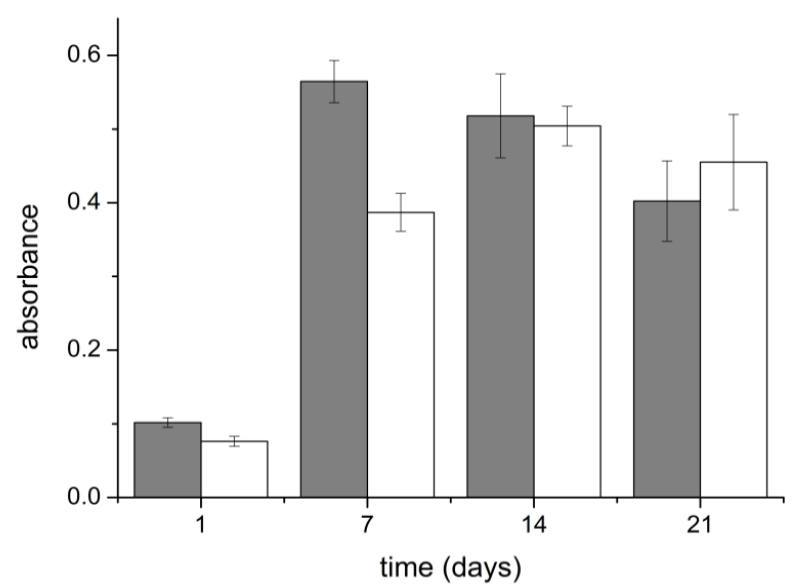

Figure 3-20. Cellular viability measured in GEL (grey bars) and composite containing 1\% of PLLA-PHEMA fibers (white bars) by MTS. 


\subsubsection{Morphology and distribution of $L 929$ cells in the composites}

Figure 3-21 shows the actin cytoskeleton of 2929 fibroblasts encapsulated in GEL and GEL+1PLLA-PHEMA at 1, 7 and 14 days of cell culture. After one day of culture (Figure 3-21a and Figure 3-21b for GEL and GEL+1PLLA-PHEMA, respectively) the density of cells inside the hydrogels is low and their shape is round, as usually reported in low stiffness hydrogels cultured for short periods of time [186]-[188]. The first differences between the two types of hydrogels can be seen in Figure 3-21c and Figure 3-21d. The GEL hydrogel is highly populated with elongated fibroblasts coexisting with some cells with a more spherical cytoskeleton (Figure 3-21c). However, fibroblasts embedded in the GEL+1PLLA-PHEMA composite are more dispersed than in pure gelatin and have a clear elongated shape typical of fibroblasts (Figure 3-21d). Finally, the pictures at 14 days of cell culture (Figure 3-21e and Figure 3-21f) corroborate that cell density in pure gelatin and in the composite with PHEMA grafted fibers is similar, which confirms the observations of the MTS assay.

The effective cellular encapsulation and growth in the two types of hydrogels confirm their viability and prove that the PHEMA grafting onto the fibers does not compromise the use of the composites as injectable materials for tissue engineering, as previously reported for pure injectable gelatin [129], [186]. 

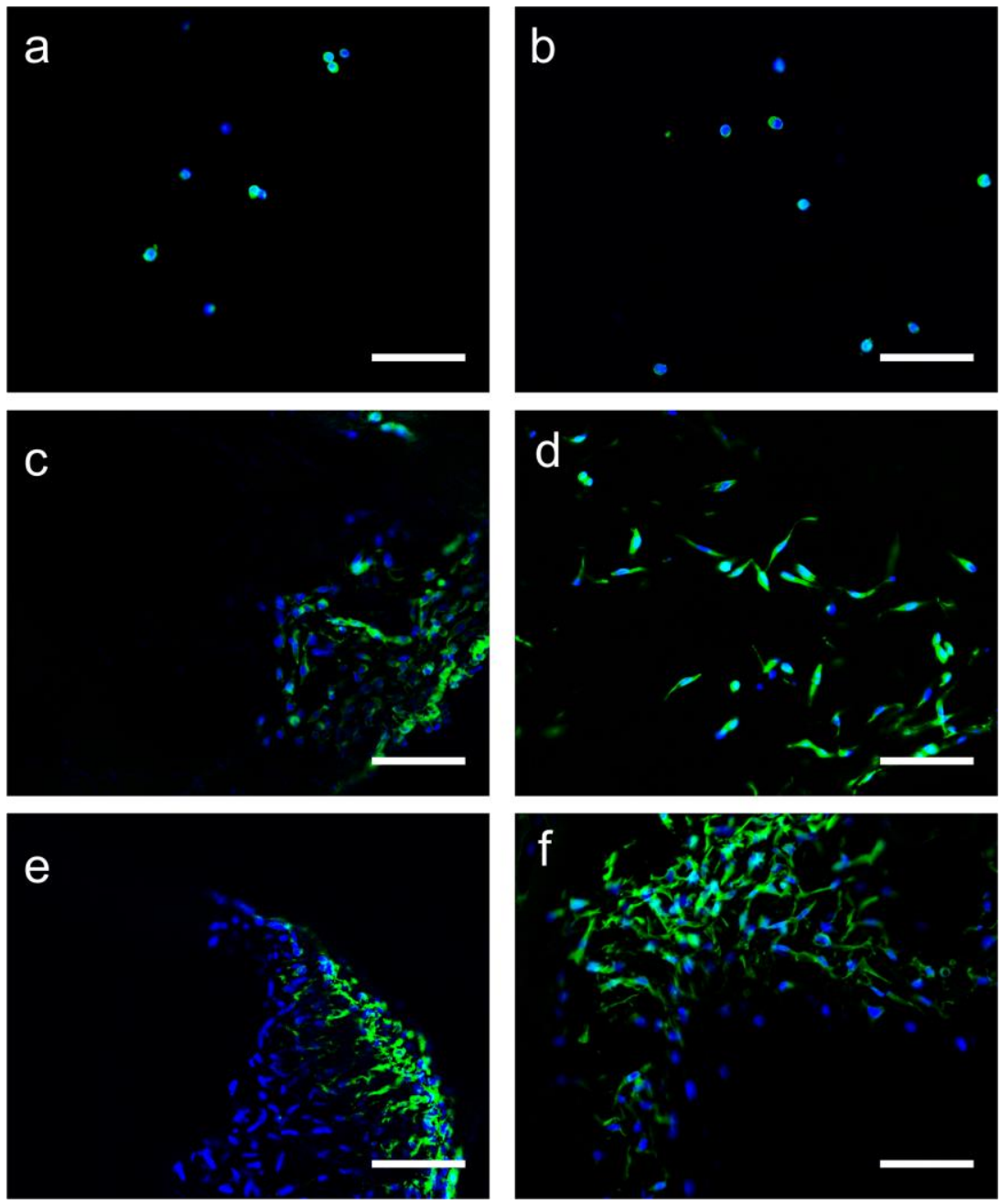

Figure 3-21. Actin (green) and nuclei (blue) stained L929 cells in GEL for (a) 1 day, (c) 7 days and (e) 14 days; and in GEL+1PLLA-PHEMA for (b) 1 day, (d) 7 days and (f) 14 days of cell culture. Scale bar $100 \mu \mathrm{m}$. 


\subsubsection{Conclusions}

Injectable fiber reinforced gelatin hydrogels were synthesized in which a very good interfacial interaction between the PLLA microfibers and the gelatin matrix was achieved via surface grafting of PHEMA chains onto the fibers. The storage moduli of the enzymatically crosslinked gels increased proportionally to the quantity of PHEMA-grafted fibers: from $184.9 \pm 32$ Pa for pure gelatin gels to $271.2 \pm 47 \mathrm{~Pa}$ when $0.5 \%$ of fibers was added, and to $535.2 \pm$ $90 \mathrm{~Pa}$ when $1 \%$ of fibers was incorporated. No significant increase in storage modulus is observed in the non-grafted fiber composites, probably due to fiber agglomeration and low interfacial interaction between the fibers and the hydrogel. Our production protocol produces loose or short microfibers which retain their shape during PHEMA grafting, enabling their incorporation into the gelatin solution containing cells and prior to the crosslinking reaction. The fibers do not compromise enzymatic crosslinking, cell encapsulation and proliferation, making these materials promising injectable systems with enhanced mechanical properties for the regeneration of soft tissues. 



\section{Chapter 4}

ECM inspired injectable gelatin-hyaluronic acid hydrogels 



\subsection{Summary*}

This chapter describes the characteristics of injectable hydrogels formed by mixtures of gelatin and high or low molecular weight hyaluronic acid (1.06 MDa and $300 \mathrm{kDa}$, respectively). Gelatin provides the RGD peptide sequences needed for cell adhesion and proliferation while hyaluronic acid gives better mechanical properties under compression, increased swelling due to its anionic character and lower degradation rates. The physical and mechanical characterization of tyramine grafted gelatin (Gel-Tyr) and hyaluronic acid (HA-Tyr) composite hydrogels (Gel/HA) was achieved with ${ }^{1} \mathrm{H}$ NMR, UV, swelling, SEM, compression assays and rheological measurements.

Cell culture studies were carried out on the Gel/HA hydrogels with low molecular weight $\mathrm{HA}$, since it shows some advantages over high molecular weight $\mathrm{HA}$; the solutions obtained are less viscous, are easier to handle and the crosslinked hydrogels are more homogenous. The influence of different Gel/HA compositions was first studied with $\mathrm{C} 2 \mathrm{C} 12$ murine myoblasts. Skeletal muscle cells have been proposed as a good cell model to study the influence of hydrogel composition and stiffness on cell behavior, since their phenotype clearly changes from undifferentiated myoblasts to differentiated myocytes, forming myotubes in short culture times [209]. Myoblast differentiation is influenced by: the presence of adhesion sequences such as RGD [210], increased matrix stiffness [211], polymer conductivity [212], high cell seeding densities [213], co-cultures of myoblasts with fibroblasts [214] or neural cells [215], and the use of micropatterns to guide myotube formation [125]. Finally, human bone marrow mesenchymal stem cells (BM-hMSCs) were embedded within the Gel/HA hydrogels and differentiation towards the chondrogenic linage was studied.

* The results presented in this chapter are in preparation to be published as three scientific articles: 
Poveda-Reyes S., Sanmartín-Masiá E. and Gallego Ferrer G. Extracellular matrix-inspired gelatin/hyaluronic acid injectable hydrogels (In preparation).

Poveda-Reyes S., Moulisova V., Sanmartín-Masiá E., SalmerónSánchez M. and Gallego Ferrer G. Myogenic Differentiation in GelatinHyaluronic Acid Injectable Hydrogels (submitted to Macromolecular Bioscience).

Poveda-Reyes S., Moulisova V., Sanmartín-Masiá E., QuintanillaSierra L., Salmerón-Sánchez M. and Gallego Ferrer G. GelatinHyaluronic Acid Hydrogels promote BM-hMSCs differentiation towards cartilage phenotype (In preparation).

\subsection{Injectable gelatin/hyaluronic acid hydrogels with high molecular weight HA}

\subsubsection{Materials and methods}

\subsubsection{Materials}

The following products were purchased from Sigma-Aldrich (Germany): Hyaluronic acid (sodium salt) from Streptococcus equi (MW $1060 \mathrm{kDa}$ ), gelatin from porcine skin (gel strength 300, Type A), and peroxidase from horseradish Type VI (HRP). All the other reagents used in this section and not described here have been detailed in 3.3.1.1. (page 96).

\subsubsection{Synthesis of HA-Tyr}

Similarly to the process used for the gelatin, hyaluronic acid was modified with tyramine (HA-Tyr). Modified HA was obtained by the reaction between carboxylic groups of HA with amine groups of tyramine as explained in [200] with some modifications.

Briefly, a solution with $150 \mathrm{mM} \mathrm{NaCl}, 0.276 \mathrm{M} \mathrm{MES}$ and $75 \mathrm{mM} \mathrm{NaOH}$ was prepared. The $\mathrm{pH}$ was adjusted at 5.75 and $0.5 \% \mathrm{w} / \mathrm{v}$ hyaluronic acid was added. The solution was stirred for 2-3 $\mathrm{h}$ at room 
temperature. Then tyramine hydrochloride was added (molar ratio 0.75:1 tyramine/carboxylic groups $(\mathrm{COOH})$ in hyaluronic acid) and stirred for 20 min to obtain good homogenization. After that, the pH was adjusted at 5.75, EDC and NHS were added (molar ratios 0.75:1 EDC/COOH and 1:10 NHS/EDC, respectively) and stirred for $24 \mathrm{~h}$ at room temperature. Non-reacted products were then removed by dialyzing against $150 \mathrm{mM} \mathrm{NaCl}$ solution for $24 \mathrm{~h}$ followed by deionized water for $24 \mathrm{~h}$. Modified HA-Tyr was lyophilized for further use.

\subsubsection{Degree of substitution}

The degree of substitution was calculated for modified Gel-Tyr (synthesized as explained in 3.3.1.3.) and HA-Tyr in order to determine the tyramine phenolic groups that had been grafted. A solution of $0.1 \%(\mathrm{w} / \mathrm{w})$ in deionized water for each material was prepared and measured at $275 \mathrm{~nm}$ with the CECIL CE9200 UV/VIS double beam spectrophotometer. Measured absorbance at this wavelength is due to the presence of phenolic groups grafted. Finally, the degree of substitution of the tyramine conjugates was obtained using a calibrated curve.

${ }^{1} \mathrm{H}$-NMR measurements were performed in $\mathrm{D}_{2} \mathrm{O}$ for the grafted and ungrafted gelatin and hyaluronic acid at a concentration of $10 \mathrm{mg} / \mathrm{mL}$ in a Spectra Spin 400 Ultrashield (Bruker). The samples were prepared in NMR sample tubes (5 $\mathrm{mm}$ in diameter) and spectra were evaluated using MestReNova software. The degree of substitution of HA-Tyr (the number of tyramine molecules per 100 repeating units) was calculated by comparing the ratio of the relative peak integrations of the phenyl protons of tyramine (peaks at 7.2 and 6.9 ppm) and the methyl protons of HA (1.9 ppm).

\subsubsection{Hydrogel formation}

Hydrogel formation takes place when phenolic groups of Gel-Tyr (synthesized as explained in 3.3.1.3.) and $\mathrm{HA}-\mathrm{Tyr}$ react in the presence of $\mathrm{HRP}$ and $\mathrm{H}_{2} \mathrm{O}_{2}$ (see Figure $4-1$ ). $1 \%$ and $2 \% \mathrm{w} / \mathrm{v}$ hydrogels 
were prepared with different Gel-Tyr and HA-Tyr (Gel/HA) volume percentages $(100 / 0,70 / 30,50 / 50,30 / 70,0 / 100)$.

First, separate solutions of Gel-Tyr and HA-Tyr were obtained by dissolving lyophilized Gel-Tyr and HA-Tyr at $1 \%$ and $2 \% \mathrm{w} / \mathrm{v}$ in CFKRB. To improve solubility, HA-Tyr solutions were maintained at 4 으 for $24 \mathrm{~h}$ and Gel-Tyr solutions were heated to $37^{\circ} \mathrm{C}$ for $30 \mathrm{~min}$, after which the different proportions of Gel/HA were mixed. Fresh solutions of HRP $(12,5 \mathrm{U} / \mathrm{mL})$ and $\mathrm{H}_{2} \mathrm{O}_{2}(20 \mathrm{mM})$ in CF-KRB were prepared. All the hydrogels were formed by homogeneously mixing $80 \% \mathrm{v} / \mathrm{v} \mathrm{Gel} / \mathrm{HA}$ solution with $10 \% \mathrm{v} / \mathrm{v} \mathrm{HRP}$ solution and finally adding $10 \% \mathrm{v} / \mathrm{v} \mathrm{H}_{2} \mathrm{O}_{2}$ solution. The samples were kept in DPBS with $0.02 \%$ $\mathrm{w} / \mathrm{v}$ sodium azide to prevent bacterial growth in a refrigerator for further characterization.

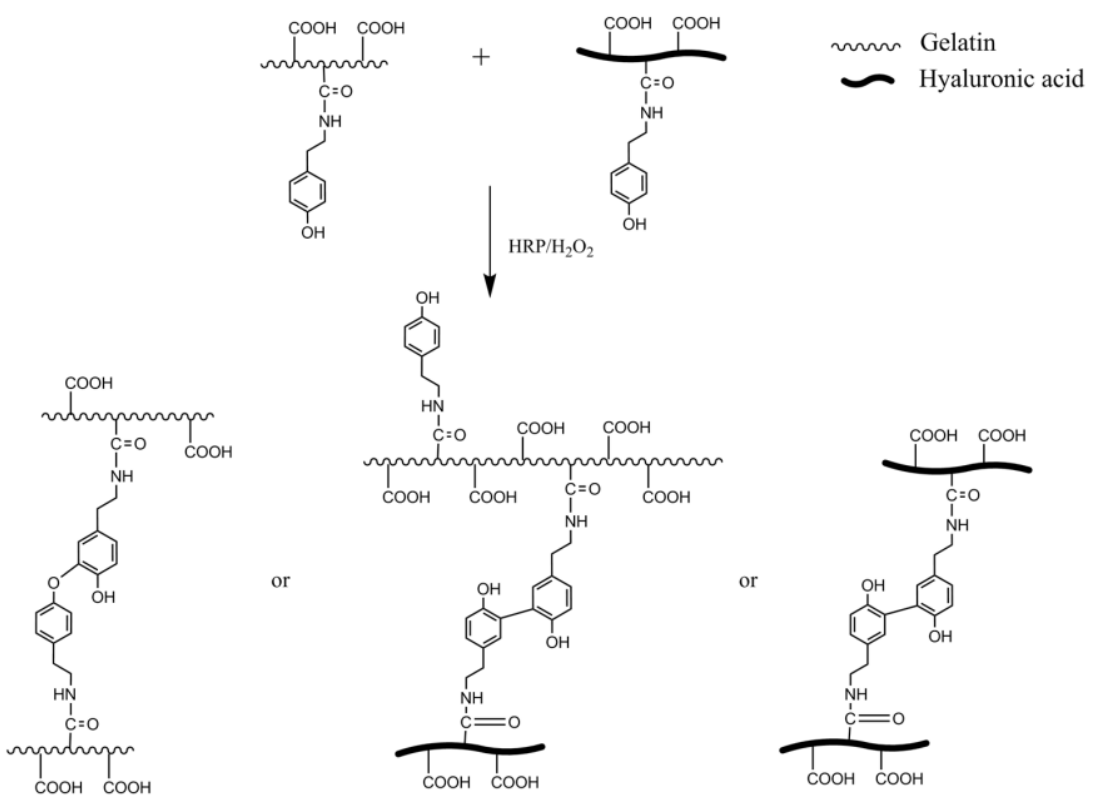

Figure 4-1. Molecular structure of enzymatically crosslinked Gel/HA hydrogels and their possible combinations. 


\subsubsection{Gelation time}

The vial tilting method was used to determine the crosslinking time. When the samples were too viscous, the initial solution remained almost motionless. To ensure that crosslinking had been achieved, a small spatula was used to detach the hydrogel from the mold, taking gelation time as the time when the hydrogel could be detached as a unit.

\subsubsection{Morphology}

Cross-sections of the different Gel/HA compositions were obtained by lyophilizing the swollen samples and cutting them with a sharp blade. The sample sections were then mounted on adhesive carbon black, sputter-coated with gold at $40 \mathrm{~mA}$ for 90 seconds and examined at an accelerating voltage of $10 \mathrm{kV}$ and $15 \mathrm{~mm}$ working distance with a Scanning Electron Microscope (JEOL JSM-5410, Japan) (SEM). ImageJ software was used to obtain the pore size from the analysis of three images for each composition.

\subsubsection{Swelling ratio}

Gel/HA hydrogels were prepared in cylindrical molds with a diameter of $8 \mathrm{~mm}$ and by pouring $300 \mu \mathrm{L}$ of total hydrogel precursors. After crosslinking, they were immersed in DPBS with $0.02 \%$ sodium azide solution at $37 \circ \mathrm{C}$ overnight to reach equilibrium and weighed $\left(m_{w}\right.$, this will be the equilibrium water absorption value). Afterwards, the samples were rinsed twice with water to remove the DPBS, and finally lyophilized to obtain the dry mass of the sample $\left(m_{d}\right)$. The equilibrium water content (EWC) was obtained using Equation 12:

$$
\operatorname{EWC}(\%)=\frac{m_{\text {water }}}{m_{d}} \cdot 100=\frac{m_{w}-m_{d}}{m_{d}} \cdot 100
$$

\subsubsection{Statistical analysis}

For statistical purposes, five replicates of each sample were used for the EWC and compression assays. The Mann-Whitney (Wilcoxon) test was used to obtain significant differences between groups $(p<0.05$ 
was considered significant). Data are given as average \pm standard deviation.

\subsubsection{Results and discussion}

\subsubsection{Degree of substitution of the tyramine conjugates and hydrogel formation}

Tyramine conjugates Gel-Tyr and HA-Tyr were obtained with a substitution degree of $1.9 \cdot 10^{-7} \mathrm{~mol} \mathrm{Tyr} / \mathrm{mg}$ Gel and $9.69 \cdot 10^{-8} \mathrm{~mol}$ $\mathrm{Tyr} / \mathrm{mg} \mathrm{HA}$, respectively. The degree of substitution of hyaluronic acid was also obtained from ${ }^{1} \mathrm{H}-\mathrm{NMR}$ measurements by comparing the ratio of the relative peak integrations of the phenyl protons of tyramine (peaks at 7.2 and $6.9 \mathrm{ppm}$ ) and the methyl protons of HA (1.9 ppm). The degree of substitution obtained for HA-Tyr was 7 (number of tyramine molecules per 100 repeating units). The presence of the tyramine molecule in the gelatin and hyaluronic acid grafted chains can be seen in the ${ }^{1} \mathrm{H}$-NMR spectrum shown in Figure $4-2 b$ and Figure $4-2 d$, respectively. The distinctive signal produced by the aromatic ring protons appears at 6.9 and $7.2 \mathrm{ppm}$.

Hydrogel mixtures of Gel/HA with different proportions by volume at $1 \%$ and $2 \%(\mathrm{w} / \mathrm{v})$ in CF-KRB were crosslinked by adding $\mathrm{HRP}$ and $\mathrm{H}_{2} \mathrm{O}_{2}$. Table 4-1 shows the gelation time of each composition.

Gelation is quite fast (between 4 and $9 \mathrm{~min}$ ) for all the hydrogels. In general, increasing polymer concentration from $1 \%$ to $2 \%(\mathrm{w} / \mathrm{v})$ lengthens the time needed to fully crosslink the hydrogel. The difference in gelation time between $1 \%$ and $2 \%$ solutions is more evident in the hydrogels with higher HA content. HA CF-KRB solutions are more viscous than Gel solutions, probably due to the high molecular weight. By increasing polymer concentration, the viscosity of the precursor solution is considerably increased, hindering $\mathrm{HRP} / \mathrm{H}_{2} \mathrm{O}_{2}$ diffusion and slowing the gelation process, so that $2 \%$ mixtures rich in hyaluronic acid need more time than the other samples to fully crosslink. It is noteworthy that $1 \% \mathrm{Gel} / \mathrm{HA}$ hydrogels 
rich in gelatin (100/0 and 70/30) do not keep their shape after gelation but are almost liquid, as if the crosslinking did not take place due to the low concentration of polymer chains. These compositions were not used for the subsequent tests.
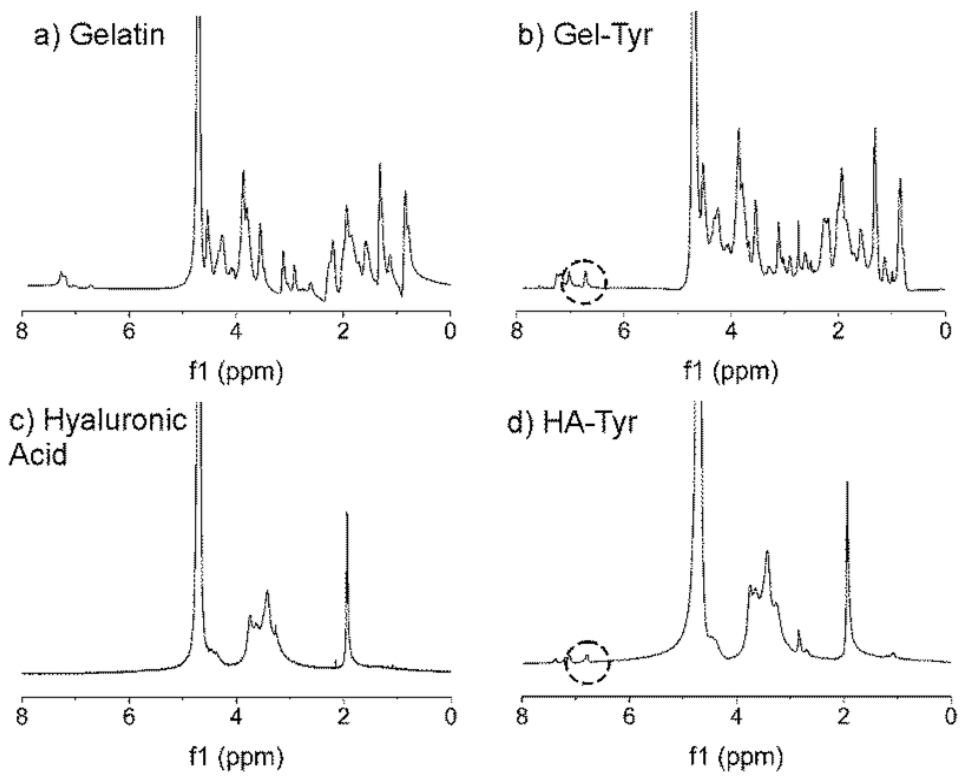

Figure 4-2. ${ }^{1} \mathrm{H}-\mathrm{NMR}$ in $\mathrm{D}_{2} \mathrm{O}$ of a) Gelatin, b) Gel-Tyr: tyramine conjugate of gelatin, c) Hyaluronic Acid and d) HA-Tyr: tyramine conjugate of hyaluronic acid. The circle indicates the presence of the aromatic protons of the tyramine molecule.

Pure HA hydrogel is transparent and pure Gel is white. As the Gel proportion is increased in the mixtures, the hydrogels become more whitish (Figure 4-3). The hydrogel mixtures at $2 \%$ have signs of phase separation, i.e. there are transparent and small white zones in the same hydrogel that correspond to $\mathrm{HA}$ and Gel zones, respectively. Macroscopically, it should be noted that the $2 \%$ are more stable and compact than the $1 \%$ hydrogels, which are softer. 
Table 4-1. Gelation time, Equilibrium Water Content (EWC) and compression Young's modulus (E) of Gel/HA hydrogels at $1 \%$ and $2 \%$ w/v.

\begin{tabular}{l|cc|cc|cc}
\hline \multirow{2}{*}{ Gel/HA } & \multicolumn{2}{|c|}{$\begin{array}{c}\text { Gelation } \\
\text { time } \\
\text { (min) }\end{array}$} & \multicolumn{2}{|c|}{ EWC (\%) } & \multicolumn{2}{|c}{ E (kPa) } \\
\cline { 2 - 7 } & $\mathbf{1 \%}$ & $\mathbf{2 \%}$ & $\mathbf{1 \%}$ & $\mathbf{2 \%}$ & $\mathbf{1 \%}$ & $\mathbf{2 \%}$ \\
\hline $100 / 0$ & - & 4 & - & $3366 \pm 635$ & - & $4.086 \pm 0.144$ \\
$70 / 30$ & - & 7 & - & $3789 \pm 781$ & - & $4.582 \pm 0.405$ \\
$50 / 50$ & 6 & 7 & $6716 \pm 1448$ & $5236 \pm 676$ & $4.078 \pm 0.234$ & $4.776 \pm 0.211$ \\
$30 / 70$ & 6 & 8 & $7798 \pm 1026$ & $5774 \pm 553$ & $4.560 \pm 0.303$ & $5.754 \pm 0.506$ \\
$0 / 100$ & 6 & 9 & $12579 \pm 1333$ & $6124 \pm 526$ & $5.984 \pm 0.421$ & $6.275 \pm 0.532$ \\
\hline
\end{tabular}

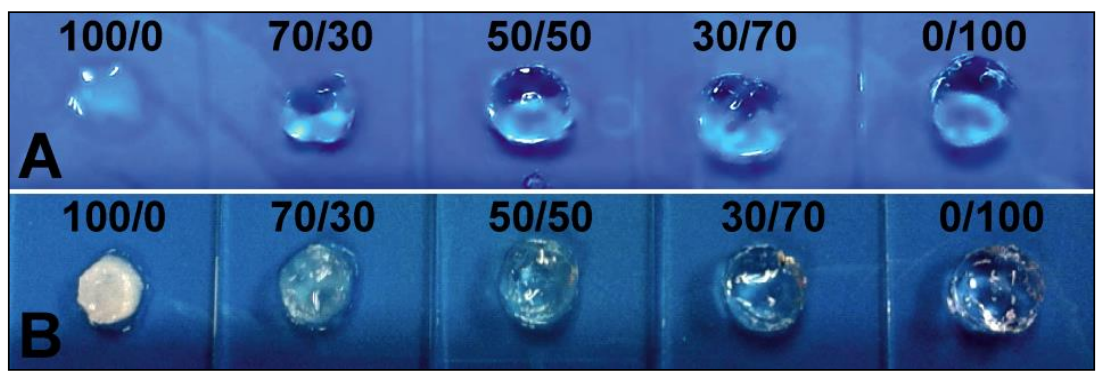

Figure 4-3. Macroscopic appearance of the hydrogels: A) 1\% Gel/HA and B) $2 \% \mathrm{Gel} / \mathrm{HA}$.

\subsubsection{Morphology}

The structure of the different hydrogels was studied by SEM in order to obtain their porous structure and their microscopic appearance. $1 \%$ Gel/HA hydrogels (Figure 4-4) present an interconnected porous structure, allowing nutrient and waste diffusion to the cells. Hydrogels with both gelatin and hyaluronic acid have a closer porous network with smaller pores than the HA sample (see Figure 4-4), a structure that is influenced by the presence of gelatin. 

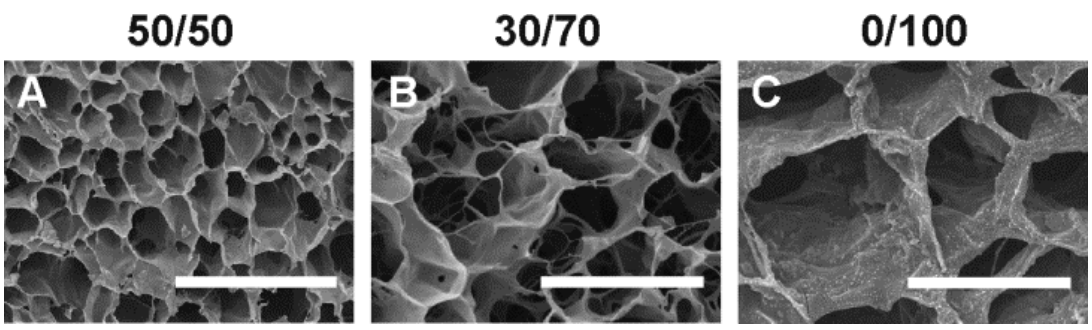

Figure 4-4. SEM images of 1\% Gel/HA hydrogels: A) 50/50, B) 30/70 and C) 0/100 Gel/HA. Scale bar $30 \mu \mathrm{m}$.
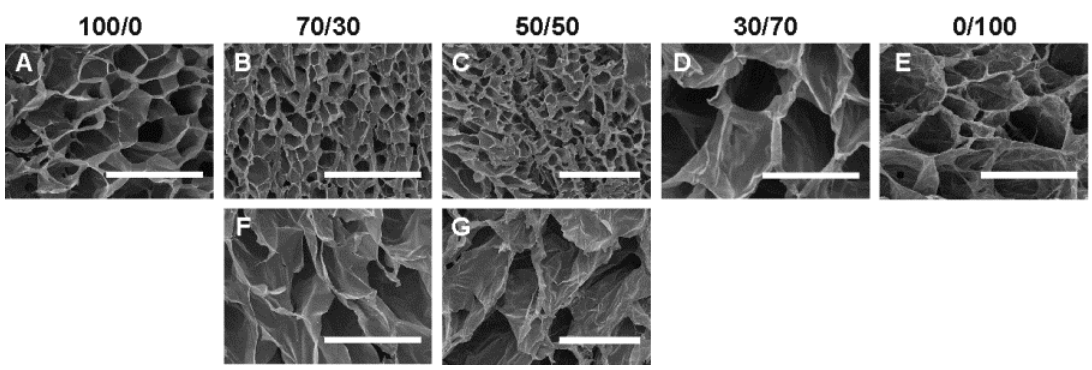

Figure 4-5. SEM images of 2\% Gel/HA hydrogels (100/0, 70/30, 50/50, 30/70 and 0/100 Gel/HA). Scale bar $60 \mu \mathrm{m}$.

For $50 / 50 \mathrm{Gel} / \mathrm{HA}$ and $\mathrm{HA}$, if the polymer concentration is increased from $1 \%$ to $2 \% \mathrm{w} / \mathrm{v}$ a similar pore size is obtained. In the case of 30/70 Gel/HA pores with larger diameters are obtained at 2\% (from 5 to $20-35 \mu \mathrm{m}$ for $1 \%$ and $2 \%$ respectively, see Figure 4-5 and Table 42). Taking the results of Table 4-2 as a whole, it seems that gelatin tends to form structures with smaller pores, while HA tends to form structures with bigger pores, a tendency previously observed by Choi et al. [91]. The value of the diameter obtained for 30/70 Gel/HA at $1 \%$ must be due to gelatin and the higher value obtained at $2 \%$ to the hyaluronic acid, since the pore size is quite similar to the value obtained for $2 \% \mathrm{HA}$.

The pore size obtained in 70/30 and 50/50 Gel/HA at 2\% (Table 4-2) is only in the area with smaller pores. The values obtained in these areas are smaller than the pore size of the gelatin sample (with 
diameter of 15-22 $\mu \mathrm{m})$. Assuming that the areas with smaller pores are rich in gelatin, the presence of hyaluronic acid appears to move the gelatin chains closer to one another and thus reduces the pore size.

\subsubsection{Swelling ratio}

Figure 4-6 shows EWC values for the Gel/HA system. Samples prepared at a concentration of $1 \% \mathrm{w} / \mathrm{v}(50 / 50,30 / 70$ and $0 / 100$ $\mathrm{Gel} / \mathrm{HA}$ ) absorb a higher amount of water than those prepared at a concentration of $2 \% \mathrm{w} / \mathrm{v}$. A more porous hydrogel is expected for less concentrated hydrogels, giving rise to hydrogels with better water retaining capacity. Compared with the work of Camci-Unal et al. that synthesized methacrylated mixtures of gelatin (GelMA) and hyaluronic acid (HAMA) and found HAMA hydrogels had a EWC of $5220 \%$ and $3900 \%$ for 1 and $2 \% \mathrm{w} / \mathrm{v}$, respectively, our HA hydrogels are able to swell higher amounts of water as EWC is $12579 \%$ and $6124 \%$ at $1 \%$ and $2 \%$, respectively. The HAMA hydrogels have a high level of methacrylation ( $80 \%)$ than our degree of tyramine substitution, being gels with higher crosslinking levels, which means lower EWC. Even though the same trend is observed, an increase in polymer concentration reduces EWC due to the higher crosslinking density and smaller pore size [122].

Pure hydrogels have very high equilibrium water sorption values (compared to dry mass), 3366\% for gelatin and $6124 \%$ for HA, which is consistent with the work of Wang et al., who obtained an HRP crosslinked gelatin hydrogel with approximately $3000 \%$ of water uptake [216]. With regard to hyaluronic acid, Fan et al. obtained 5\% w/v HA-Tyr hydrogels with an EWC of $3800 \%$, somewhat lower than the one obtained in the present study, due to the higher polymer concentration and the use of $20 \mathrm{U} / \mathrm{mL} \mathrm{HRP}$ and $10 \mathrm{mM} \mathrm{H}_{2} \mathrm{O}_{2}$, values that are higher than the $1.25 \mathrm{U} / \mathrm{mL} \mathrm{HRP}$ and $2 \mathrm{mM} \mathrm{H}_{2} \mathrm{O}_{2}$ used in this work [217]. Lee et al. obtained $1.75 \% \mathrm{w} / \mathrm{v}$ HA-Tyr hydrogels crosslinked with lower HRP concentration $(0.124 \mathrm{U} / \mathrm{mL})$ and varying the $\mathrm{H}_{2} \mathrm{O}_{2}$ concentration, obtaining EWC ranging from $7000 \%$ to 
$3000 \%$ by increasing the $\mathrm{H}_{2} \mathrm{O}_{2}$ content from 160 to $728 \mu \mathrm{M}$, which indicates that the swelling capacity was reduced due to the formation of more crosslinks [136].

Table 4-2. Pore diameter of Gel/HA hydrogels at different compositions. Hydrogels were synthesized using solutions of $1 \%$ and $2 \% \mathrm{w} / \mathrm{v}$.

\begin{tabular}{c|cc}
\hline Concentration w/v & Gel/HA & Diameter $(\boldsymbol{\mu m})$ \\
\hline \multirow{2}{*}{$1 \%$} & $50 / 50$ & $6-15$ \\
$30 / 70$ & $5-8$ \\
& $0 / 100$ & $12-39$ \\
\hline \multirow{2}{*}{$2 \%$} & $100 / 0$ & $15-22$ \\
& $70 / 30$ & $8-12^{\text {a }}$ \\
& $50 / 50$ & $6-10^{\text {a }}$ \\
& $30 / 70$ & $20-35$ \\
& $0 / 100$ & $20-35$
\end{tabular}

\footnotetext{
a Two pore areas can be distinguished in these compositions. The written pore size corresponds to the area with smaller pores. The pore size of the area with bigger pores could not be calculated since in most of them is difficult to distinguish one pore from the other.
}

At both polymer concentrations there is an increase in EWC with higher HA content, a trend also reported by other authors [69], [91], [120]. For the same mass of both polymers, HA has a higher hygroscopic nature, i.e. it has more water-binding sites than gelatin, improving its water retention due to the formation of more hydrogen bonds with water [120]. Choi et al. synthesized Gel and HA sponges crosslinked with EDC capable of absorbing from 30 to 40 times the weight of the dry sponge [91]. However, Zhoe et al. obtained Gel/HA sponges crosslinked with EDC, but with higher EWC, ranging between 40.5 for gelatin and 75.2 for hyaluronic acid [120], values that are more similar to those obtained here. 


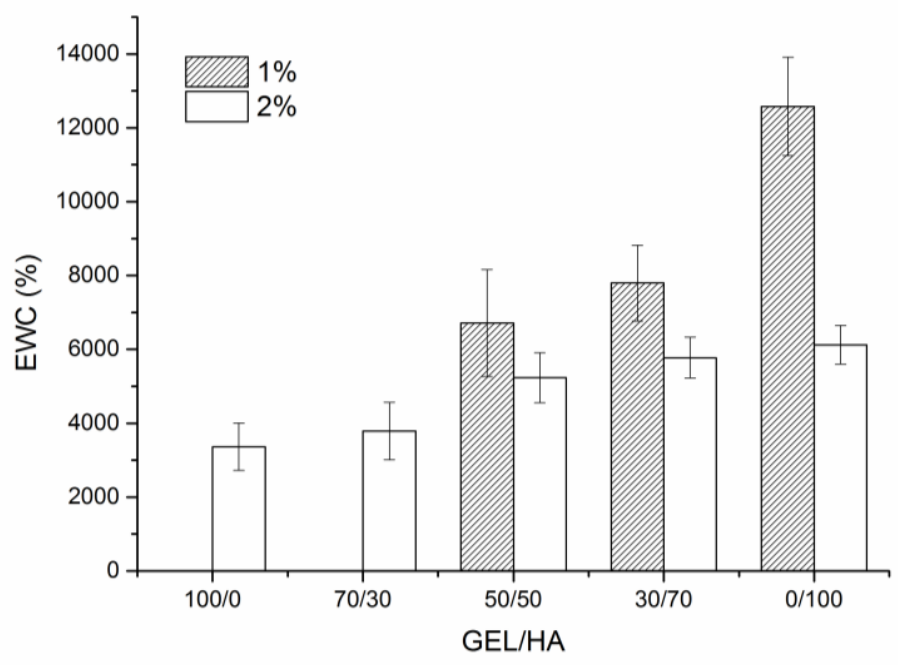

Figure 4-6. Equilibrium Water Content (EWC) percentages for the different Gel/HA samples studied at $1 \%$ and $2 \% \mathrm{w} / \mathrm{v}$.

Water retention in the hydrogels is also responsible for hydrogel reinforcement, with water diffusion being hindered due to hydrogen bonding with polymeric chains and crosslinking points that increase the stress needed for deformation of the hydrogel under compression. Even considering the differences between the water absorption values for gelatin and hyaluronic acid, both hydrogels have high water retention and are thus suitable for use in soft tissue engineering.

\subsubsection{Mechanical properties}

Five replicates of each composition for both concentrations were tested $24 \mathrm{~h}$ after being in DPBS as described in 3.2.1.6. The stressstrain curves of the unconfined compression tests for representative samples of each composition can be seen in Figure 4-7. 

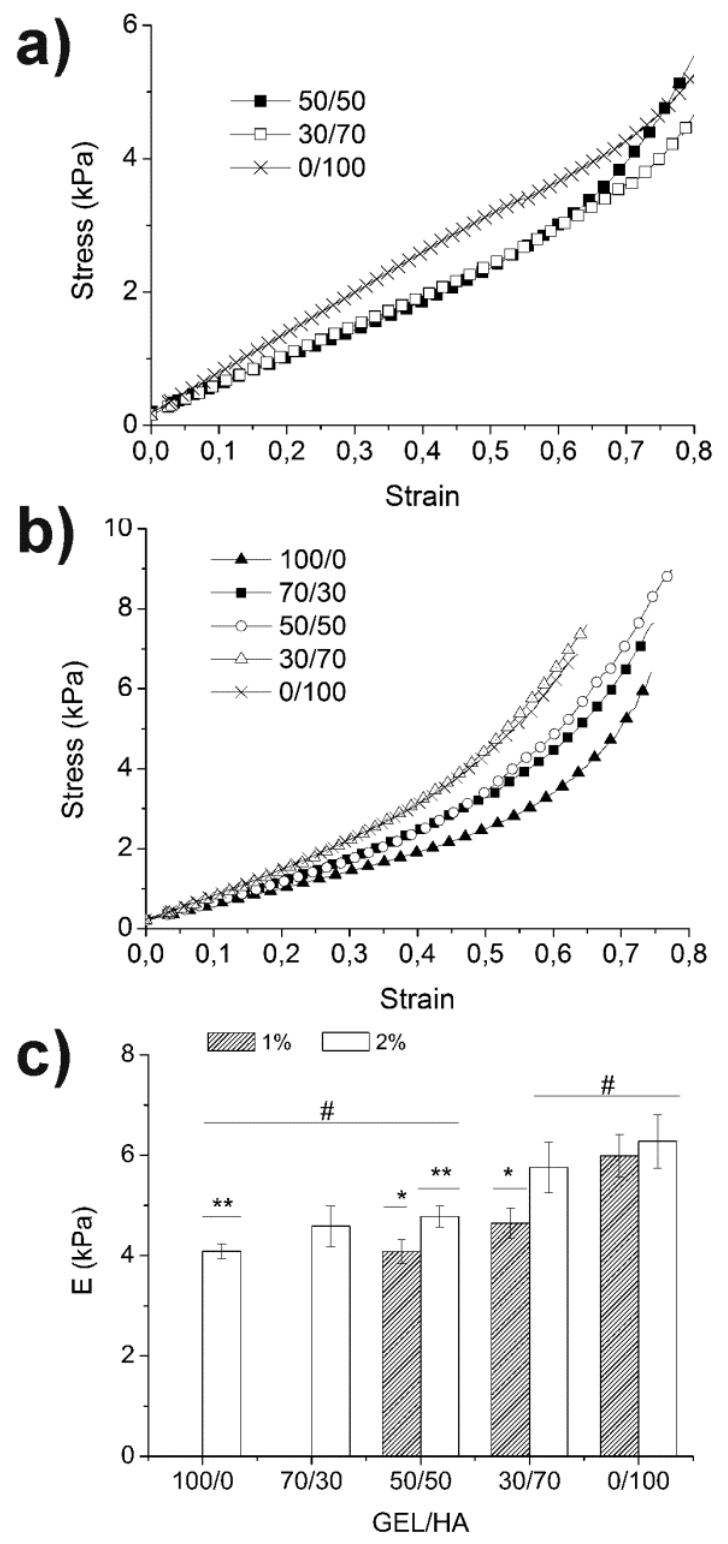

Figure 4-7. Stress-strain curves for (a) 1\% GEL/HA and (b) $2 \%$ GEL/HA hydrogels. (c) Comparison of Young's modulus (E) obtained for each composition. *,** and \# denote significant differences between groups. 
For both the $1 \%$ and $2 \%$ hydrogel concentrations, as HA content increases the stress needed to deform the hydrogels to a given strain also increases, which indicates the reinforcement provided by including HA in the mixtures. Pure gelatin thus has significantly worse mechanical properties than hyaluronic acid, yet its sorption capacity is also lower than HA, due to its lower molecular weight, the thinner walled structure with low mechanical strength [212] and the lower water interaction, which facilitates water expulsion in deformation.

For $1 \% \mathrm{Gel} / \mathrm{HA}$ (Figure 4-7a), the curves obtained for the $50 / 50$ and $30 / 70$ mixtures are similar, while the curve for pure $\mathrm{HA}(0 / 100)$ has a steeper slope for small deformations. On the other hand, for $2 \%$ Gel/HA hydrogels (Figure 4-7b), the HA reinforcement is noticeable at low concentrations and the 70/30 and 50/50 samples present similar curves with higher stresses for all strains than pure Gel. In these samples reinforcement is notable in the mixtures with the highest HA content (30/70), which mostly overlaps the pure HA curve.

Young's modulus (E) was calculated for all the samples as the slope of the stress-strain curve in the initial linear region (up to $20 \%$ strain). The values for each composition can be seen in Figure 4-7c. The $2 \%$ have higher $\mathrm{E}$ values than the $1 \%$ hydrogels, which is consistent with the increased water sorption capacity of the latter and the increased water permeability. Due to the low concentration of polymer chains these hydrogels have higher sorption capacity but worse mechanical properties. For $2 \% \mathrm{Gel} / \mathrm{HA}$ hydrogels Young's modulus increases monotonously with the proportion of $\mathrm{HA}$, from 4,086 $\pm 0,144 \mathrm{kPa}$ for pure gelatin to $6,275 \pm 0,532 \mathrm{kPa}$ for HA hydrogel (Table 4-1), giving statistically significant differences intermediate values for the different Gel/HA mixtures. For $1 \% \mathrm{Gel} / \mathrm{HA}$ hydrogels, significant differences were obtained between 50/50, 30/70 and 0/100 hydrogels, with $E$ increasing from $4,078 \pm 0,234 \mathrm{kPa}(50 / 50)$ to 5,984 $\pm 0,421 \mathrm{kPa}$ for pure HA hydrogel $(0 / 100)$. 
These results confirm our hypothesis that adding HA to the Gel hydrogel improves gelatin's mechanical properties in proportion to the HA added.

\subsubsection{Conclusions}

Gel/HA hydrogels were synthesized at $1 \%$ and $2 \% \mathrm{w} / \mathrm{v}$ with an interconnected porous structure to allow nutrient and waste diffusion for cell survival. The obtained in situ formed gelatin has smaller pores than the hyaluronic acid hydrogel. In the case of the formation of mixtures of gelatin and hyaluronic acid (70/30, 50/50 and 30/70 Gel/HA), different zones of each component were obtained according to the different pore sizes observed. The presence of HA interferes with gelatin crosslinking and pushes its chains closer to one another forming a structure with smaller pores than those obtained in pure gelatin. As regards the time needed for hydrogel crosslinking by $\mathrm{HRP} / \mathrm{H}_{2} \mathrm{O}_{2}$, increasing polymer or $\mathrm{HA}$ concentration also increases gelation time.

Material properties are also influenced by the polymer concentration and the Gel/HA ratio. If the Gel/HA concentration or the amount of $\mathrm{HA}$ is increased, a higher Young's modulus is also obtained, with values from $4 \mathrm{kPa}$ for Gel to $6,3 \mathrm{kPa}$ for $\mathrm{HA}$, with intermediate values for the mixtures. The EWC assay shows that reducing polymer concentration increases the hydrogel's EWC, due to the larger porosity. Moreover, increasing the HA concentration in the hydrogel also raises the EWC, since it has more hydrophilic groups than gelatin to form secondary bonds. Although mixtures have demonstrated higher mechanical properties than pure gelatin, they are not homogeneous due to the high viscosity of HA, and the systems presented signs of phase separation. In the following section, optimized mixtures of Gel/HA are presented, using hyaluronic acid solutions with lower viscosity, obtained after reducing its molecular weight. 


\subsection{Injectable gelatin/hyaluronic acid hydrogels with low molecular weight HA. Physico-chemical characterization.}

\subsubsection{Materials and methods}

\subsubsection{Materials}

Dialysis tubing (3500 MWCO) and hydrochloric acid were purchased by Sigma-Aldrich (USA). The suppliers of all the other materials have been described in 4.2.1.

\subsubsection{Preparation of Low Molecular Weight (LMW) HA by acid degradation}

Hyaluronic acid of low molecular weight (around 320,000 Da) was obtained from acid degradation of the high molecular weight hyaluronic acid [218]. Briefly, $500 \mathrm{mg}$ of high molecular weight HA were dissolved in $500 \mathrm{~mL}$ of $\mathrm{HCl}$ at $\mathrm{pH} 0.5$, and the solution was stirred for $24 \mathrm{~h}$ at $37^{\circ} \mathrm{C}$ for $\mathrm{HA}$ degradation. The reaction was stopped by adjusting the $\mathrm{pH}$ to 7 with $1 \mathrm{~N} \mathrm{NaOH}$. The obtained solution was dialyzed (with a dialysis tube of $3500 \mathrm{MWCO}$ ) against distilled water for 4 days and then lyophilized to dry it.

\subsubsection{Preparation of Gelatin and Hyaluronic Acid tyramine grafting}

Gelatin grafting with tyramine was performed as previously explained (see the Materials and methods section of 3.3.).

For the tyramine grafting with the LMW HA, $100 \mathrm{mg}$ hyaluronic acid $(0.5 \% \mathrm{w} / \mathrm{v})$ was then dissolved in $20 \mathrm{~mL} 150 \mathrm{mM} \mathrm{NaCl}$ with $1.08 \mathrm{~g}$ MES and $75 \mathrm{mM} \mathrm{NaOH}$ (pH 5.75). $86.54 \mathrm{~g}$ of tyramine hydrochloride (molar ratio 2:1 Tyr/COOH groups in $\mathrm{HA}$ ) was added and the solution was stirred until full dissolution. The $\mathrm{pH}$ was adjusted at 5.75 and 
47.77 mg EDC and $2.87 \mathrm{mg}$ NHS were added (molar ratios 1:2 EDC/Tyr and 1:10 NHS/EDC). The obtained solution was stirred for 24 $\mathrm{h}$ in order to obtain the tyramine grafting and was then dialyzed $24 \mathrm{~h}$ with $150 \mathrm{mM} \mathrm{NaCl}$ and another $24 \mathrm{~h}$ against deionized water, changing the dialysis solution three times each day. Finally, the tyramine grafted hyaluronic acid solution was lyophilized for further use.

To determine the quantity of phenol groups, due to the grafting of tyramine in Gel and HA polymeric chains, the absorbance of $0.1 \%$ w/w aqueous solution of grafted Gel and HA was measured at 275 $\mathrm{nm}$ with UV beam spectrophotometer, obtaining values of $1.9 \cdot 10^{-7}$ mol Tyr/ mg gelatin and $9.69 \cdot 10^{-8} \mathrm{~mol}$ Tyr/mg HA, for tyramine grafted Gel and HA respectively. The content of introduced phenol groups was calculated from a calibration curve of known percentages of tyramine hydrochloride in distilled water. ${ }^{1} \mathrm{H}-\mathrm{NMR}$ measurement for the tyramine grafted HA of low molecular weight shows a degree of substitution of $6 \%$.

\subsubsection{Gel/HA hydrogels preparation}

$2 \% \mathrm{w} / \mathrm{v}$ hydrogels were prepared with different Gel-Tyr and HA-Tyr (Gel/HA) volume percentages $(100 / 0,70 / 30,50 / 50,30 / 70,0 / 100)$ as previously described in 4.2.1.4. but with LMW HA instead of the high molecular weight $\mathrm{HA}$.

\subsubsection{Physical and chemical characterization}

Scanning Electron Microscopy images, EWC and unconfined compression tests were performed as previously explained in 4.2.1.

\subsubsection{Mechanical characterization (rheology)}

Rheological experiments were performed on a strain-controlled AR2000ex rheometer (TA Instruments). A solvent trap geometry of parallel plates (made of nonporous stainless steel, diameter $=20$ $\mathrm{mm}$ ) was used to reduce solvent loss during the experiment. The gap between the plates was around $1200 \mu \mathrm{m}$. Sample temperature was 
controlled and maintained by a Peltier device and measurements were always carried out at $37^{\circ} \mathrm{C}$.

All measurements were made in a shear deformation mode. First of all, an oscillatory time sweep was selected to follow the gelation dynamics of the samples. The mixtures of gelatin, hyaluronic acid and HRP enzyme were arranged on the plate at $37^{\circ} \mathrm{C}$. After adding the correct amount of $\mathrm{H}_{2} \mathrm{O}_{2}$ to initiate the reaction, measurements were recorded (after the 10 - $15 \mathrm{~s}$ required to lower the plate). The time evolution of the rheological parameters was recorded for a period of 20 minutes. Strain and frequency were selected at $1 \%$ and $1 \mathrm{~Hz}$, respectively.

After the reaction time $(20 \mathrm{~min})$ the samples had been crosslinked and were kept on the plate for two further measurements. First, the range of strain amplitudes at which the gels exhibit a linear region of viscoelasticity was determined. A dynamic strain sweep (with amplitudes ranging between $0.01 \%$ and $15 \%$ ) was carried out at a frequency of $1 \mathrm{~Hz}$ to measure the dynamic shear modulus as a function of strain. Secondly, in order to determine the dependence of the dynamic shear modulus and the loss factor on frequency, dynamic frequency sweep tests were performed. A frequency sweep between $0.1 \mathrm{~Hz}$ and $10 \mathrm{~Hz}$ at a fixed strain (corresponding to the hydrogel linear region) was chosen.

The following data were obtained from the rheological measurements: storage modulus $\left(G^{\prime}\right)$, loss modulus $\left(G^{\prime \prime}\right)$, complex modulus magnitude $\left(\left|G^{*}\right|\right.$, a measure of the hydrogel stiffness: $\left.\left|G^{*}\right|^{2}=\left(G^{\prime}\right)^{2}+\left(G^{\prime \prime}\right)^{2}\right)$, and loss factor $\left(\tan \delta \equiv\left(G^{\prime \prime}\right) /\left(G^{\prime}\right)\right.$, a measure of the internal energy dissipation, where $\delta$ is the phase angle between the applied stimulus and the corresponding response) as a function of reaction time, strain amplitude or frequency.

\subsubsection{Enzymatic Degradation Study}

After hydrogel synthesis, the hydrogels were left overnight in DPBS with $0.02 \% \mathrm{w} / \mathrm{v}$ sodium azide to reach equilibrium swelling. The in 
vitro degradation of Gel/HA hydrogels was subsequently performed by incubating the hydrogels with hyaluronidase and collagenase at $37^{\circ} \mathrm{C}$. Cylindrical samples ( $7 \mathrm{~mm}$ diameter and $280 \mu \mathrm{L}$ volume) were incubated in $10 \mathrm{U} / \mathrm{ml}$ of hyaluronidase and $3 \mathrm{U} / \mathrm{mL}$ of collagenase solutions in DPBS with $0.5 \% \mathrm{w} / \mathrm{v}$ sodium azide at $37^{\circ} \mathrm{C}$. Five replicates were conducted for each composition and time point.

Degradation was followed by the mass loss of the hydrogel as a function of time. The initial swollen mass $\left(m_{S_{0 t}}\right)$ was noted and measured at the different time points $\left(m_{S_{t}}\right)$, which provided mass loss by Equation 13:

$$
\% \text { mass loss }=\frac{m_{S_{0 t}}-m_{S_{t}}}{m_{S_{0 t}}}
$$

The equilibrium water content (EWC) of the hydrogel swollen in DPBS with $0.02 \% \mathrm{w} / \mathrm{v}$ sodium azide overnight, which was used for comparison after formation and after reaching 20-30\% degradation, was obtained by Equation 12 .

\subsubsection{Statistical analysis}

For the statistical studies Statgraphics software was used and the Mann-Whitney (Wilcoxon) test was performed to find any statistical differences between the groups at $95 \%$ confidence intervals $(p<$ 0.05).

\subsubsection{Results and discussion}

\subsubsection{SEM images and Equilibrium water content}

Figure 4-8a shows the microscopic morphology of the hydrogels with different Gel/HA compositions. No signs of phase separation were observed, confirming our hypothesis: the reduction of HA molecular weight allowed the preparation of homogenous mixtures. Hydrogel pore size increases with higher HA content (see Table 4-3). A statistical analysis shows two differentiated groups, one formed by 100/0, 70/30 and 50/50 Gel/HA hydrogels, with no significant 
statistical differences between the compositions and average pore size of around $17 \mu \mathrm{m}$. The other group is formed by 30/70 and $0 / 100$ Gel/HA, which have higher hyaluronic acid content and larger pore size (26 and $37 \mu \mathrm{m}$, respectively). Both show statistically significant differences with the higher gelatin content group.
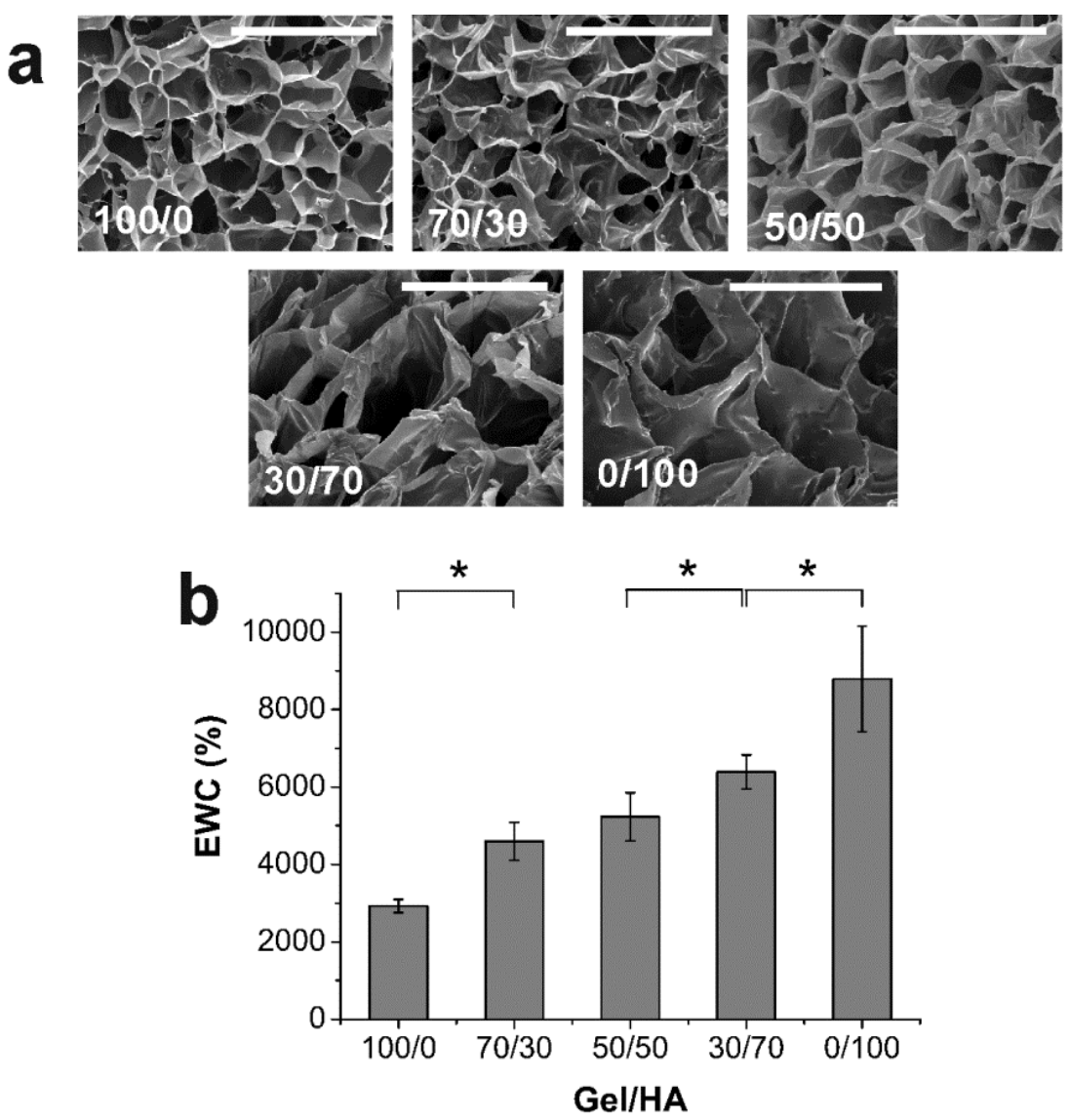

Figure 4-8. a) Scanning Electron Microscope images of the five different Gel/HA hydrogels. Scale bar corresponds to $60 \mu \mathrm{m}$. b) Equilibrium water content of the different Gel/HA compositions in $D P B S$ at $37^{\circ} \mathrm{C} .{ }^{*}$ indicates statistical significance between groups. 
The quantity of DPBS absorbed by the different Gel/HA compositions was measured as described in 4.2.1.7. and the obtained values can be seen in Figure $4-8 \mathrm{~b}$. The amount of water within the hydrogels increases with higher quantities of HA in the hydrogel composition, which corresponds to the larger pore size obtained in the SEM analysis.

A EWC of $2924 \%$ for gelatin and of $8790 \%$ for HA were obtained, the different mixtures having intermediate values (see Table 4-3). The statistical analysis indicates significant differences (Figure 4-8b) between all the samples except between the 70/30 and 50/50 hydrogels.

Raising the HA content increases pore size and the quantity of water that the hydrogels are able to absorb. This higher water retention in HA has been explained mainly by its hygroscopic nature, obtaining hydrogels with a larger pore size when the percentage of $\mathrm{HA}$ increases [91], [120] and/or less crosslinking [69], in this case by reduced tyramine grafting, which would lead to a greater distance between crosslinking points and more space to lodge water within its polymeric structure.

\subsubsection{Compression tests}

Unconfined compression tests in immersion were performed on the different Gel/HA samples. A representative stress-strain curve for each composition was selected in order to better understand hydrogel behavior (see Figure 4-9a). A linear behavior can be seen in all the samples at up to $30 \%$ strain. In this range water flows out of the hydrogel when compression forces are increased. After this point, the slope gradually changes and the hydrogel water content is probably reduced, with the mechanical properties of the polymeric matrix being more noticeable. The depicted curves show that for hydrogels with a higher HA content, higher stress or force is needed to deform the material at a given strain. 
The compressive Young's moduli for the samples immersed in DPBS are given in Figure 4-9b, calculated from the initial linear region of the stress-strain curves at up to $20 \%$ strain. Higher HA content in the Gel/HA mixtures increases the compressive strength of the hydrogels and higher Young's moduli were obtained.

a)

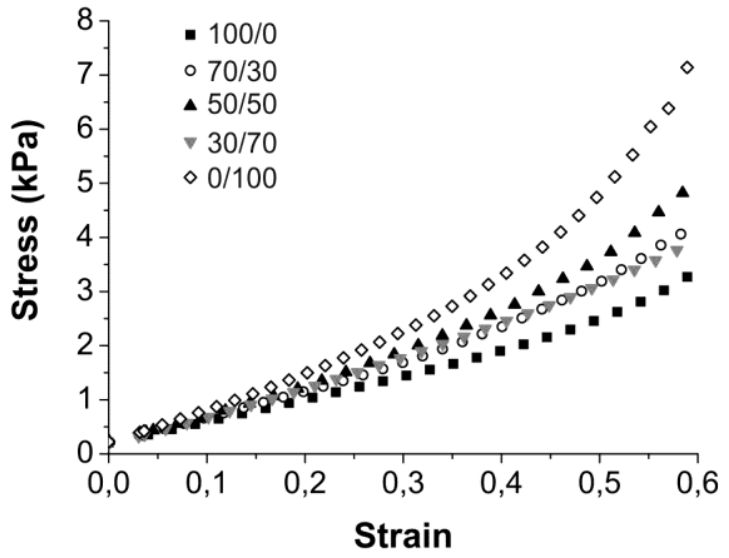

b)

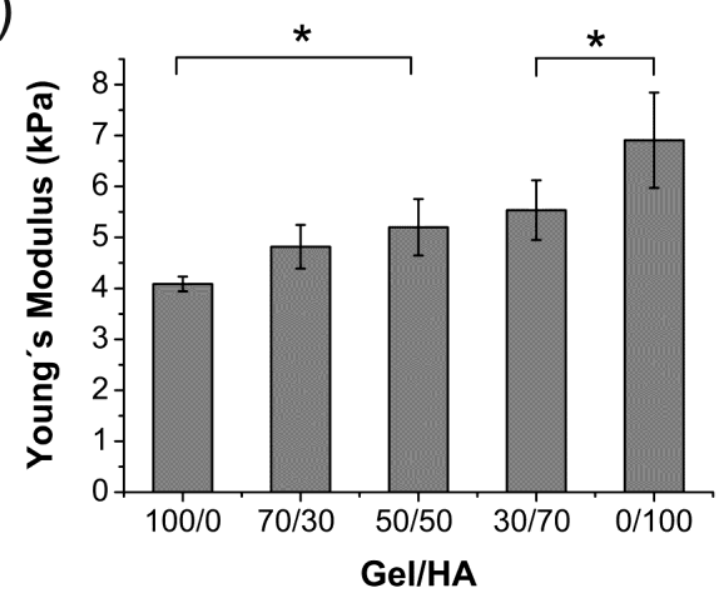

Figure 4-9. a) Stress vs. strain curves of the Gel/HA hydrogels under compression and b) Young's modulus from 0 to $20 \%$ strain of the samples immersed in DPBS, $n=5$. * indicates statistical significance between groups. 
Table 4-3. Properties of the different Gel/HA compositions (compressive Young's modulus, EWC and pore size).

\begin{tabular}{cccc}
\hline $\begin{array}{c}\text { Gel/HA } \\
\text { Hydrogel }\end{array}$ & $\begin{array}{c}\text { Young's } \\
\text { Modulus }(\mathbf{k P a})\end{array}$ & $\begin{array}{c}\text { EWC } \\
(\%)\end{array}$ & $\begin{array}{c}\text { Pore size } \\
(\boldsymbol{\mu m})\end{array}$ \\
\cline { 1 - 3 } $100 / 0$ & $4.1 \pm 0.1$ & $2924 \pm 169$ & $18.2 \pm 2.9$ \\
$70 / 30$ & $4.8 \pm 0.4$ & $4597 \pm 489$ & $16.2 \pm 3.6$ \\
$50 / 50$ & $5.2 \pm 0.5$ & $5232 \pm 620$ & $17.4 \pm 5.6$ \\
$30 / 70$ & $5.5 \pm 0.6$ & $6390 \pm 439$ & $25.9 \pm 12.5$ \\
$0 / 100$ & $6.9 \pm 0.9$ & $8790 \pm 1363$ & $37.2 \pm 8.9$ \\
\hline
\end{tabular}

A value of $4 \mathrm{kPa}$ versus $6.9 \mathrm{kPa}$ was calculated for pure Gel and HA, respectively, the different Gel/HA hydrogels having intermediate values. Significant differences were obtained between $0 / 100 \mathrm{Gel} / \mathrm{HA}$ and all the other groups and between 100/0 Gel/HA with 50/50 and 30/70 Gel/HA.

The typical Young's moduli (E) values of natural hydrogels are in the order of magnitude of a few $\mathrm{kPa}$ [116], [142], [219]. In the present study we obtained hydrogels with $\mathrm{E}$ values ranging from 4 to $7 \mathrm{kPa}$ (see Table 4-3). These values are higher than for pure gelatin with increased HA content but are still within the range of soft hydrogels. In another study with enzymatically crosslinked HA-Tyr hydrogels, Lee et al. found that the increase in $\mathrm{H}_{2} \mathrm{O}_{2}$ concentration produced an increase in its storage modulus, which has been correlated with the higher crosslinking density of the hydrogel when higher oxidation is achieved by the $\mathrm{H}_{2} \mathrm{O}_{2}$ [134]. The reaction mechanism produced by the $\mathrm{HRP} / \mathrm{H}_{2} \mathrm{O}_{2}$ coupling starts with the oxidation of the HRP by the $\mathrm{H}_{2} \mathrm{O}_{2}$, which afterwards oxidizes the tyramine groups of the hyaluronic acid or the gelatin chain, see Figure 4-1 [134]. Then the percentage of tyramine groups forming crosslinking points will depend not only on the quantity of tyramine moieties grafted onto the gelatin or 
hyaluronic acid backbone, but also on the amount of $\mathrm{H}_{2} \mathrm{O}_{2}$. In the above study, HA-Tyr hydrogels were obtained with a storage modulus of $3 \mathrm{kPa}$, this value being lower than the value obtained in the present study. This was due to their using an HA with lower molecular weight (90 kDa compared to the $300 \mathrm{kDa}$ hyaluronic acid used in this study) and also to lower $\mathrm{HRP}$ and $\mathrm{H}_{2} \mathrm{O}_{2}$ concentrations.

Toh et al. also studied the effect of varying $\mathrm{H}_{2} \mathrm{O}_{2}$ concentration from 500 to $1000 \mu \mathrm{M}$ and obtained a compressive Young's modulus from 5 to $11 \mathrm{kPa}$ and a reduction of the swelling ratio $\left(\mathrm{m}_{\mathrm{w}} / \mathrm{m}_{\mathrm{d}}\right)$ from 43 to 33 , which confirms that higher $\mathrm{H}_{2} \mathrm{O}_{2}$ concentrations promote hydrogel crosslinking [111]. In this study, $\mathrm{HRP}$ and $\mathrm{H}_{2} \mathrm{O}_{2}$ concentrations were kept constant in order to observe the effect of the two hydrogel components, gelatin and the hyaluronic acid. The higher Young's modulus with the higher HA content in the hydrogel could be explained by the formation of a higher crosslinked network due to the presence of more tyramine groups grafted onto the HA backbone, or could be due to the material's own mechanical properties and the stronger retention of water. Therefore, the hyaluronic acid hydrogel has a higher modulus than the gelatin hydrogel because of its innate physico-chemical characteristics; most importantly, its high water retention capacity keeps water inside the hydrogel when an external force is applied, which improves the mechanical properties of hydrogel compression. Another characteristic that may have an influence on its mechanical properties is its molecular weight, which is $300 \mathrm{kDa}$ for HA-Tyr versus $50 \mathrm{kDa}$ for Gel-Tyr (these values were obtained by GPC, results not shown). Kim et al. showed the influence of using 10 and $50 \mathrm{kDa}$ hyaluronic acid on the mechanical properties and found higher complex and elastic modulus with the higher molecular weight [220].

The mechanical properties of the Gel/HA mixtures exhibit higher compressive strength than pure gelatin $(100 / 0 \mathrm{Gel} / \mathrm{HA})$ and at the same time have gelatin's important advantage of providing the RGD sequence for cell adhesion [221], [222]. Incorporating the RGD sequence into different hydrogel matrices has been reported to 
induce myoblast adhesion, proliferation and differentiation [130], [210], [213] as well as to improve adhesion to other cell types [223], [224].

\subsubsection{Mechanical properties (rheology)}

An oscillatory time sweep was carried out to determine gelation dynamics. The storage modulus with reaction time is shown in Figure 4-10a. The loss modulus did not show any changes in these tests (it remained constant with a value around $1 \mathrm{~Pa}$, regardless of the hybrid gel composition, results not shown). The reaction time was swept for $20 \mathrm{~min}$ at $37^{\circ} \mathrm{C}$ and the in situ sample gelation took place throughout this period.

We used the stabilization time of the storage modulus to compare the gelation dynamics of our hybrid gels as this is an important parameter in the hydrogel implantation process.

As can be seen in Figure 4-10a, the fastest stabilization time is for pure gelatin (100/0), while hyaluronic acid is the slowest. When gelatin is present in a hybrid hydrogel composition, $G^{\prime}(t)$ is found to change rapidly in short times, but less so in longer times. By the end of the measurement, the storage modulus of pure gelatin is totally stabilized, while a small, but non-zero slope is observed in $\mathrm{G}^{\prime}$ for every hybrid gel and pure hyaluronic acid. The dependence of the slope, $\mathrm{dG}^{\prime}(\mathrm{t}) / \mathrm{dt}$, on reaction time was calculated and the stabilization time was estimated as the time when the $G^{\prime}(t)$ slope became constant. The fastest dynamics was observed in pure gelatin, and stabilization time is longer when the HA ratio is increased in the hybrids (see Table 4-4). 


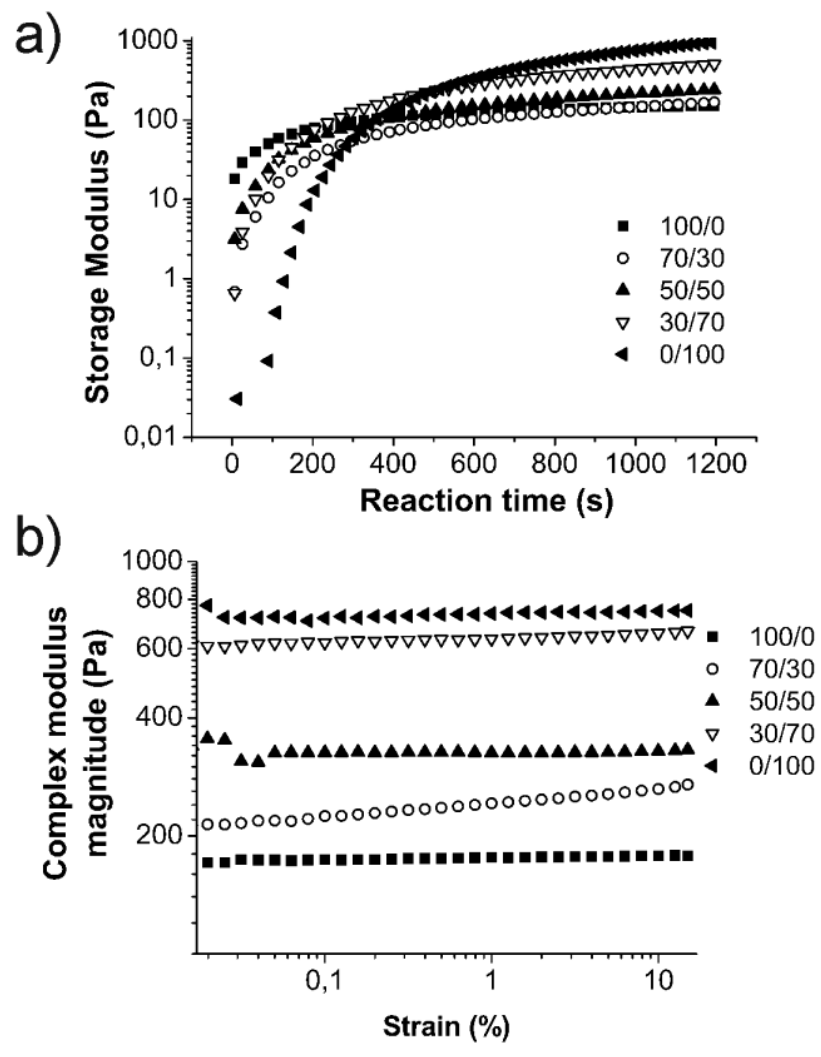

Figure 4-10. a) Crosslinking kinetics represented with storage modulus as a function of reaction time at a frequency of $1 \mathrm{~Hz}$ and $1 \%$ strain; b) complex modulus magnitude $\left(\left|G^{*}\right|\right)$ of crosslinked hydrogels as a function of the strain at a frequency of $1 \mathrm{~Hz}$. All measurements were carried out at $37^{\circ} \mathrm{C}$. Each curve corresponds to the average of three different samples. 

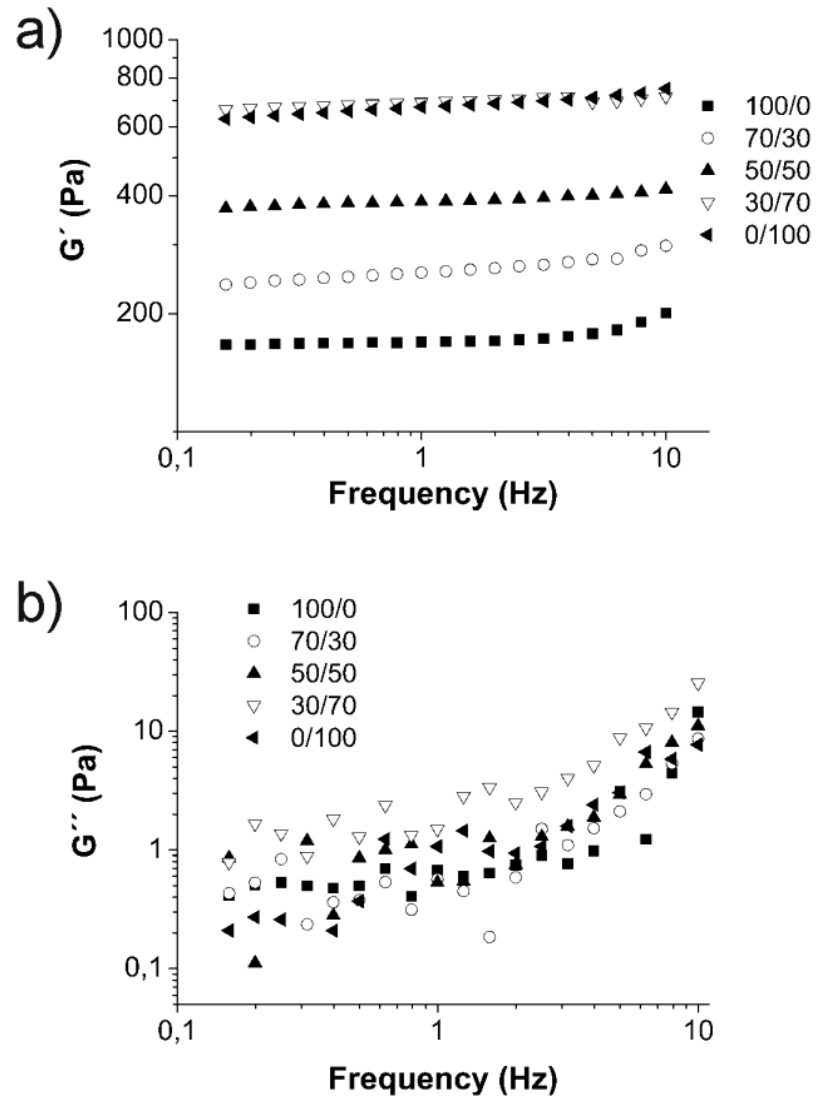

Figure 4-11. Evolution of a) the storage ( $\left.G^{\prime}\right)$ and b) loss moduli ( $\left.G^{\prime \prime}\right)$ as a function of the frequency of crosslinked hydrogels (1\% strain). All measurements were carried out at $37^{\circ} \mathrm{C}$. Each curve corresponds to the average of three different samples.

After 20 min of measurements a strain sweep was carried out to obtain the hydrogel linear viscoelastic range [204]-[206]. No noticeable change was observed in $\left|G^{*}\right|$ with the strain amplitude (Figure 4-10 throughout the whole amplitude strain range swept in any of the gels. As can be seen, $\left|G^{*}\right|$ increases with the HA ratio, which has been reported previously in similar systems [121]. As a trade-off between linearity and noise, the subsequent dynamic frequency sweep tests were performed for the $1 \%$ strain. 
Table 4-4. Stabilization time estimated from the time evolution of the storage modulus for the hybrid hydrogels at different compositions. The results obtained for pure gelatin (100/0 Gel/HA) and pure hyaluronic acid $(0 / 100 \mathrm{Gel} / \mathrm{HA})$ (control samples) have been included for comparison.

\begin{tabular}{cc}
\hline $\begin{array}{c}\text { Sample Composition } \\
\text { (Gel/HA) }\end{array}$ & $\begin{array}{c}\text { Stabilization time } \\
\text { (min) }\end{array}$ \\
\hline $100 / 0$ & $1.87 \pm 0.23$ \\
$70 / 30$ & $2.25 \pm 0.15$ \\
$50 / 50$ & $2.19 \pm 0.23$ \\
$30 / 70$ & $2.41 \pm 0.07$ \\
$0 / 100$ & $4.54 \pm 0.08$ \\
\hline
\end{tabular}

In Figure 4-11a and Figure 4-11b the dependence of $G^{\prime}$ and $G^{\prime \prime}$ on frequency has been plotted for the hybrid gels, respectively. The storage modulus is the dominant contribution to $\left|G^{*}\right|$, since $G^{\prime}>>$ $\mathrm{G}^{\prime \prime}$ in all the gels, as has also been reported in thiolated gelatinhyaluronic acid hydrogels [121] and in oxidized hyaluronic acidgelatin-adipic acid dihydrazide hydrogel [119]. No significant dependence of $G^{\prime}$ on the frequency is observed for the experimental range of frequency swept. Again, the higher the ratio of HA in the hybrid gel the higher the storage modulus. No noticeable change is found in the loss modulus (with a value around $1 \mathrm{~Pa}$, regardless of the gel composition) until $2-3 \mathrm{~Hz}$, but it does increase at higher frequencies.

Focusing on a fixed frequency, $\mathrm{G}^{\prime}, \mathrm{G}^{\prime \prime}$ and $\tan \delta$ values at $1 \mathrm{~Hz}$ have been included in Table 4-5. The value of $\mathrm{G}^{\prime}$ at $1 \mathrm{~Hz}$ appears in the range of several hundreds of $\mathrm{Pa}$. As far as the loss factor is concerned, an extremely low value (about $0.1^{\circ}$ ) is found for all the hybrid gels, indicating a highly elastic energy storing capacity. 
Table 4-5. Storage $\left(G^{\prime}\right)$, and loss $\left(G^{\prime \prime}\right)$ moduli, loss factor (tan $\left.\delta\right)$, and phase angle $(\delta)$ evaluated at a frequency of $1 \mathrm{~Hz}$, using a $1 \%$ strain, and at $37^{\circ} \mathrm{C}$ for the hybrids with different compositions. The results obtained for pure gelatin $(100 / 0 \mathrm{Gel} / \mathrm{HA})$ and pure hyaluronic acid (0/100 Gel/HA) (control samples) have been included for comparison.

\begin{tabular}{ccccc}
\hline $\begin{array}{c}\text { Sample } \\
\text { Composition } \\
\text { (Gel/HA) }\end{array}$ & $\mathbf{G}^{\prime}(\mathrm{Pa})$ & $\mathbf{G}^{\prime \prime}(\mathrm{Pa})$ & $\tan \delta$ & $\left.\delta \mathbf{(}^{\circ}\right)$ \\
\hline $\mathbf{1 0 0 / 0}$ & $172.0 \pm 38$ & $0.9 \pm 0.3$ & $5.2 \times 10^{-3}$ & $\sim 0.3$ \\
& & & $\pm 1.8 \times 10^{-3}$ & \\
$\mathbf{7 0 / 3 0}$ & $277.4 \pm 32$ & $0.5 \pm 0.06$ & $1.8 \times 10^{-3}$ & $\sim 0.1$ \\
& & & $\pm 4 \times 10^{-4}$ & \\
$\mathbf{5 0 / 5 0}$ & $366.0 \pm 28$ & $0.5 \pm 0.004$ & $1.4 \times 10^{-3}$ & $\sim 0.1$ \\
& & & $\pm 1 \times 10^{-4}$ & \\
$\mathbf{3 0 / 7 0}$ & $690.4 \pm 85$ & $1.5 \pm 0.3$ & $2.2 \times 10^{-3}$ & $\sim 0.1$ \\
& & & $\pm 5 \times 10^{-4}$ & \\
$\mathbf{0 / 1 0 0}$ & $789.5 \pm 220$ & $1.0 \pm 0.09$ & $1.3 \times 10^{-3}$ & $\sim 0.1$ \\
& & & $\pm 4.5 \times 10^{-4}$ & \\
\hline
\end{tabular}

\subsubsection{Enzymatic Degradation of Gel/HA hydrogels}

The degradation dynamics of Gel/HA hydrogels was studied by enzymatic degradation with a $10 \mathrm{U} / \mathrm{ml}$ solution of hyaluronidase and $3 \mathrm{U} / \mathrm{mL}$ of collagenase in DPBS, these concentrations being within the range typically used in the bibliography [90], [91], [114], [131], [225], [226]. A mixture of both enzymes was used to study degradation, since it has been demonstrated that the combination of both produces higher degradation rates than when only one is used [69]. Degradation kinetics was studied as the mass lost with time of immersion in the degradation solution (see Figure 4-12a). Gelatin hydrogel degrades very rapidly; after 7 hours there is no hydrogel left, while a longer time ( 5 days) is needed for hyaluronic acid. This 
trend has previously been reported by other authors [69]. Gel/HA hybrids need intermediate times for their degradation. 70/30 and $50 / 50 \mathrm{Gel} / \mathrm{HA}$ hydrogels show a degradation profile similar to the gelatin hydrogel, and 30/70 fully degrades after $28 \mathrm{~h}$. These results show the high degradation dependence of the hydrogels on gelatin, since even small amounts of gelatin (30\%) accelerate degradation, in comparison to pure HA. In the hybrids, gelatin is firstly degraded by collagenase, leaving spaces between some of the hyaluronic acid chains and improving the accessibility of hyaluronidase to degrade this component faster than bare HA hydrogel (0/100).

In order to better understand how degradation influences hydrogel structure, the EWC of the different hydrogels at $20-30 \%$ degradation was compared with the EWC of the hydrogels with no degradation (Figure 4-12b).

EWC is generally increased in all the compositions studied after degradation. This fact could indicate a homogenous degradation throughout the hydrogel, known as bulk degradation [136]. Internal hydrogel degradation creates chain cleavage in the peptide bond (for gelatin)[91] and in the $\beta-1-4$ glycosidic linkages (for hyaluronic acid) [102], [136], causing reduced hydrogel crosslinking that creates loosened networks and more hydroxyl groups capable of absorbing more water. Also, since gelatin degrades first, it will leave gaps or small pores that will increase enzyme diffusion and hydrogel degradation. On the other hand, no change in EWC was obtained after $30 \%$ degradation (typical of surface degradation) in the hyaluronic acid hydrogel, probably due to the difficulty of enzyme diffusion inside the hydrogel. 
a)

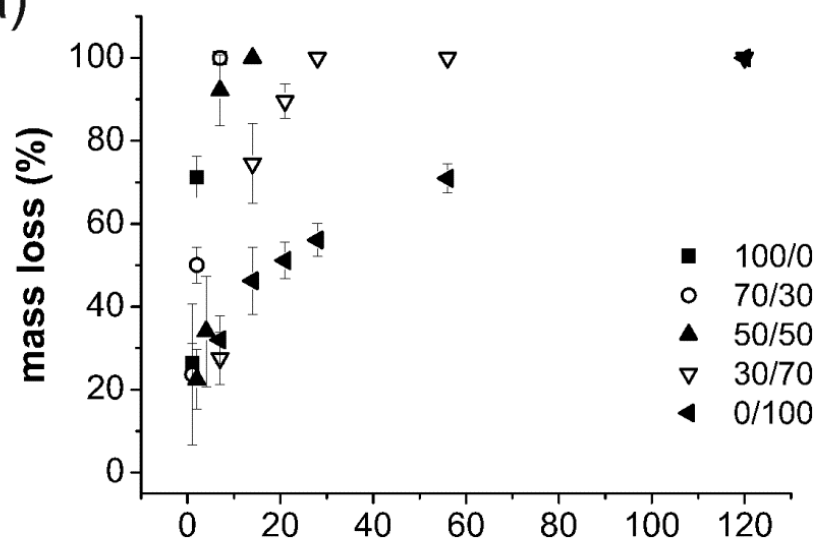

b)

Time (h)

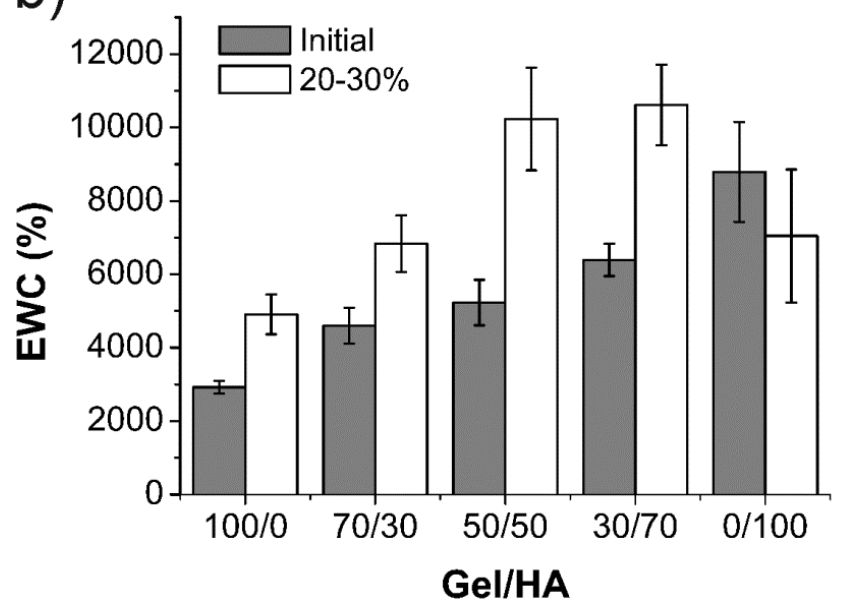

Figure 4-12. Degradation study: a) percentage of mass lost with time for each Gel/HA hydrogel and b), equilibrium water content (EWC) of the Gel/HA hydrogels just after synthesis (Initial) and after a $20-30 \%$ of degradation of the matrix (2030\%). 


\subsubsection{Conclusions}

A series of Gel/HA (100/0, 70/30, 50/50, 30/70 and 0/100 v/v) injectable hydrogels were synthesized and the proportions of both components were varied in order to study the effects on their material properties. The Gel/HA injectable hydrogels needed 2-5 min for crosslinking. Hydrogels with higher HA:Gel ratio (higher HA content) had higher G' values, ranging from $172 \mathrm{~Pa}$ for Gel to $789 \mathrm{~Pa}$ for HA. The presence of higher hyaluronic acid content in the hydrogel composition was found to yield higher Young's and shear loss moduli and higher EWC. This effect could be due to hyaluronic acid's higher molecular weight, the presence of fewer crosslinking points and the affinity of water to be retained during compression.

Enzymatic degradation in a collagenase-hyaluronidase medium showed higher degradation rates for gelatin, which influences cell ECM remodelling. 


\subsection{C2C12 differentiation in gelatin/hyaluronic acid injectable hydrogels}

\subsubsection{Materials and methods}

\subsubsection{Materials}

For cell culture experiments, C2C12 cells, primary antibody for myosin (MF-20b, $800 \mu \mathrm{g} / \mathrm{mL}$ ) was from Developmental Studies Hybridoma Bank (DSHB, USA) and secondary antibody rabbit antimouse Cy3 was purchased from Jackson Immunoresearch (USA). Mounting reagent Vectashield with DAPI (4,6-diamidino-2phenylindole) was from Vector Laboratories Inc (USA). Goat serum, trypsin/EDTA, P/S, Triton X-100 and 37\% formaldehyde were from Sigma-Aldrich (USA). FBS, DMEM with high glucose and ITS-X were purchased from Gibco and NucBlue was from Molecular Probes, (ThermoFisher Scientific, USA). BODIPY FL Phallacidin was obtained from Life Technologies (USA) and OCT compound mounting medium for cryotomy was purchased from VWR (USA).

All the other reagents needed for the synthesis of Gel/HA injectable hydrogels with low molecular weight HA (LMW HA) were detailed in 4.3.1.

\subsubsection{Hydrogel formation for cell culture experiments}

$2 \% \mathrm{w} / \mathrm{v}$ Gel and $2 \% \mathrm{w} / \mathrm{v}$ LMW HA solutions were prepared by dissolving the lyophilized powder in DMEM with $1 \% \mathrm{P} / \mathrm{S}, 24 \mathrm{~h}$ at $4^{\circ} \mathrm{C}$ for $\mathrm{HA}$ and $30 \mathrm{~min}$ at $37^{\circ} \mathrm{C}$ for Gel. $12.5 \mathrm{U} / \mathrm{mL} \mathrm{HRP}$ solution was then added to the prepared solutions at a volume ratio of $10 / 80(\mathrm{~mL}$ of HRP solution/mL Gel or HA solution) and the obtained mixture was filtered through a $0.22 \mu \mathrm{m}$ syringe filter under the cell culture hood for sterilization. Solutions of different proportions (100/0, 70/30, $50 / 50,30 / 70,0 / 100 \mathrm{v} / \mathrm{v}$ ) of Gel+HRP and HA+HRP were then 
prepared. Cells were then added to each Gel/HA mixture. Finally, 45 $\mu \mathrm{L}$ of the Gel/HA cell suspension were crosslinked with $5 \mu \mathrm{L}$ of $20 \mathrm{mM}$ $\mathrm{H}_{2} \mathrm{O}_{2}$ on each well of the $\mathrm{p} 48$ cell culture plate.

\subsubsection{Myosin differentiation}

C2C12 myoblasts were expanded in the presence of DMEM medium with $20 \% \mathrm{FBS}$ and $1 \% \mathrm{P} / \mathrm{S}$ (growth medium), at $37^{\circ} \mathrm{C}$ and $5 \% \mathrm{CO}_{2}$ in an incubator. At $60 \%$ confluence, the cells were released from the culture flask with $4 \mathrm{~mL}$ of trypsin/EDTA for $10 \mathrm{~s}$, the trypsin was removed and the flask was incubated for $3 \mathrm{~min} .10 \mathrm{~mL}$ of growth medium were added to stop trypsin activity, and centrifuged at 1400 rpm for $4 \mathrm{~min}$. Cells were resuspended in DMEM with $1 \% \mathrm{P} / \mathrm{S}$, counted, the required quantity for each Gel/HA solution was taken, centrifuged at $1400 \mathrm{rpm}$ for $2 \mathrm{~min}$ and resuspended in the Gel/HA with HRP solution at a cell density of $8 \times 10^{6}$ cells $/ \mathrm{mL}(400,000$ cells per hydrogel, passage 3 ). The Gel/HA cell suspension was crosslinked as described above and left in the incubator at 37 ㅇ $\mathrm{C}$ and $5 \% \mathrm{CO}_{2}$ for

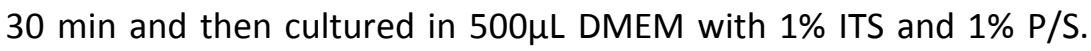
Triplicates of each composition were produced. Cell medium was changed every 2 days.

After 4 days, samples were washed with DPBS, fixed with $4 \%$ formaldehyde for $15 \mathrm{~min}$ and washed again with DPBS.

For 3D images, samples were permeabilized with $0.1 \%$ Triton $\mathrm{X}-100$ in PBS for 20 min at room temperature, washed with PBS, blocked with $5 \%$ Goat Serum in PBS (blocking buffer) for $1 \mathrm{~h}$ and washed with PBS. Then 1:250 of the primary antibody MF-20 was added in blocking buffer for sarcomeric myosin staining for $1 \mathrm{~h}$, washed with PBS and 1:200 of rabbit anti-mouse Cy3 secondary antibody in blocking buffer was added for $1 \mathrm{~h}$, washed and kept in PBS. Images were taken in the fluorescent microscope Zeiss Observer Z1_AX10 at different heights, and the $3 \mathrm{D}$ image was taken as $z$ projection of the images at the different heights using ImageJ. 
Hydrogels were sectioned to obtain better quality fluorescent pictures with less background than 3D images. For this, formaldehyde fixed samples were soaked in $30 \% \mathrm{w} / \mathrm{v}$ sucrose in DPBS overnight, included in $\mathrm{OCT}$, frozen with liquid nitrogen and kept at $-80^{\circ} \mathrm{C}$. Finally, samples were cut at $40 \mu \mathrm{m}$ with the cryostat Leica CM 1860 UV. Sections were similarly stained, but after adding the secondary antibody the sample was stained with BODIPY FL Phallacidin 1:100 for 20 min, washed and mounted in Vectashield with DAPI. CellC software was used for cell counting and to determine the percentage of differentiation.

\subsubsection{Cell contractility inhibition}

C2C12 cells were cultured in 100/0 Gel/HA and 70/30 Gel/HA hydrogels as described previously. Differentiation medium (DMEM with $1 \%$ ITS and $1 \% \mathrm{P} / \mathrm{S}$ ) was used as culture medium for $3 \mathrm{~h}$ and then changed for differentiation medium (DM) with $10 \mu \mathrm{M}$ Blebbistatin, which acts as a contractility inhibitor, $(\mathrm{DM}+\mathrm{Cl})$ for 4 days. Triplicates for each composition of the 100/0 and 70/30 Gel/HA hydrogels were cultured in this medium ( $3 \mathrm{~h}$ in $\mathrm{DM}$ and 4 days in $\mathrm{DM}+\mathrm{Cl}$ ) and in $\mathrm{DM}$ as control. The medium was changed every 2 days.

Immunofluorescence of hydrogels sections were carried out as described previously. Differences in hydrogel diameter were measured and the shrinkage was calculated according to Equation 14:

$$
\text { Shrinkage }(\%)=\frac{\emptyset_{0}-\emptyset_{f}}{\emptyset_{0}} \cdot 100
$$

where $\emptyset_{0}$ is the initial diameter, and $\emptyset_{f}$ is the diameter after 4 days of culture. 


\subsubsection{Statistical analysis}

For statistical purposes, three replicates of each sample were used for the cell culture experiments. The Mann-Whitney (Wilcoxon) test was used to obtain significant differences between groups $(p<0.05$ was considered significant). Data are given as average \pm standard deviation.

\subsubsection{Results and discussion}

Hybrids of gelatin and hyaluronic acid with various proportions were synthesized in order to improve cell adhesion and differentiation. Hyaluronic acid is a polysaccharide with no adhesive domains (e.g. RGD), which means it does not allow integrin mediated myoblast adhesion and further differentiation into myotubes. Gelatin is a protein substrate with adhesion domains but with very low mechanical stability, which cannot withstand traction forces exerted by encapsulated cells and the gel shrinks. Hydrogels combining both molecules are mimetic extracellular matrix materials with improved properties. To our knowledge, this is the first time that the differentiation potential of in situ crosslinked mixtures of Gel/HA has been studied, showing promising results.

\subsubsection{Myogenic differentiation}

Figure 4-14 shows the $z$ projections of stained sarcomeric myosin in the different Gel/HA compositions. Even those with only $30 \%$ gelatin in their composition promoted myotube formation whereas the cells in the HA hydrogel stayed round and no myotubes were formed. 


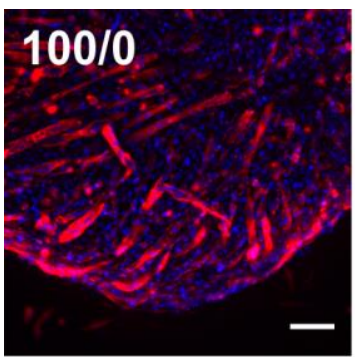

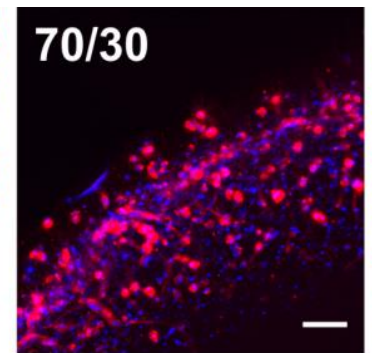

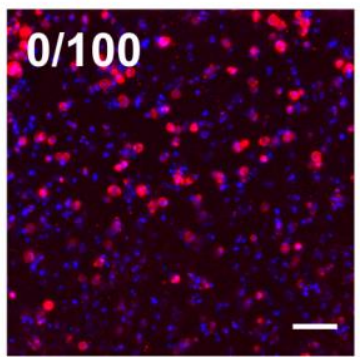

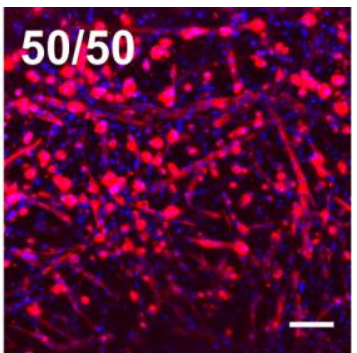

Myosin:DAPI

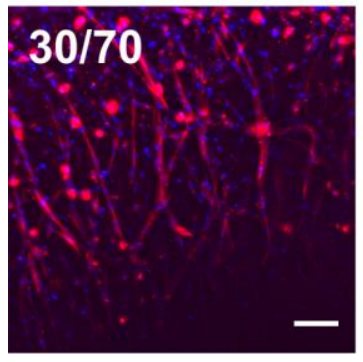

Figure 4-13. Differentiation of C2C12 cells, as indicated by sarcomeric myosin in Gel/HA hydrogels. Images were obtained as the $z$ projections of images at different heights. Scale bar $100 \mu \mathrm{m}$.

Figure $4-14 b$ shows the percentage of cells expressing sarcomeric myosin (shown in the figure as percentage of differentiation). This graph shows higher expression at the periphery of the hydrogels, mainly due to higher nutrient availability and diffusion.

Only isolated cells were observed in HA, which implies lack of cell differentiation and myotube organization. Cells located in the center of the hydrogel formed myotubes in the three Gel/HA mixtures analyzed. The high proliferation of cells in the gelatin hydrogel and the substantial shrinkage of the hydrogel itself did not allow myotube formation in the interior of Gel hydrogels. This effect can be elucidated from Figure 4-14b, in which the quantity of cells per area is represented, showing a large number of cells at the center of the gelatin hydrogel.

Figure 4-15 shows the cell morphology in the different Gel/HA hydrogels at high magnification. Expression of myosin was marked in red and formation of myotubes could be seen in all the compositions, except for the hyaluronic acid hydrogel. 

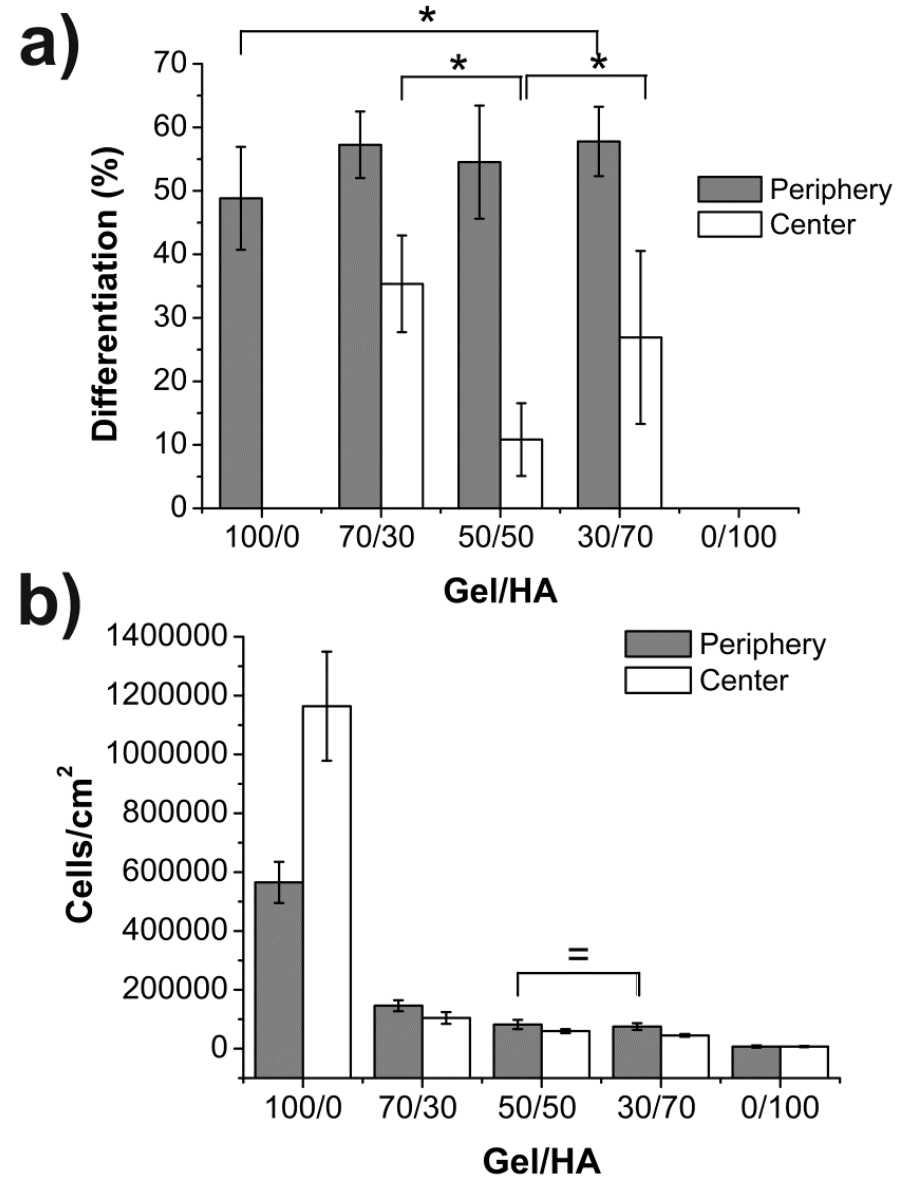

Figure 4-14. a) Percentage of cell differentiation and b) total number of cells in the different hydrogel compositions. ${ }^{*}$ and $=$ symbols indicate statistical significance and no-statistical significance between groups, respectively.

It can be clearly seen that cell differentiation happened mainly in the outer regions of the gelatin hydrogels, while the cell population was high at the center but no myosin expression was revealed (see Figure 4-14a and Figure 4-14b). In 70/30, 50/50 and 30/70 Gel/HA hybrids, cells expressing myosin appeared throughout the entire hydrogel, although more myotubes were seen at the periphery (see Figure 414a), whose area was larger in the mixtures than in the gelatin hydrogel. 
On the other hand, no myosin and no myotubes were formed in the hyaluronic acid hydrogel. The cells remained rounded inside the hydrogel and almost no proliferation was observed. These results indicate the lack of $\mathrm{C} 2 \mathrm{C} 12$ cell adhesion to this substrate and thus its incapacity for $\mathrm{C} 2 \mathrm{C} 12$ differentiation.

Table 4-6 shows the percentage differentiation obtained for the five Gel/HA hydrogel compositions. First of all, as expected, the lack of adhesion sites in HA [224] did not allow cell adhesion and rounded cells were seen inside the gel which proliferated slowly and formed clusters, as previous studies have reported with mesenchymal stem cells or neural precursor cells [227]-[229] and no myotube formation was achieved. For $100 / 0 \mathrm{Gel} / \mathrm{HA}, 49 \%$ differentiation was obtained at the hydrogel periphery but no differentiation occurred in the center due to hydrogel shrinkage, an effect that could be due to cell-matrix traction or contractile forces [213], [230]-[232]. The reduced hydrogel size meant that the central cells were very close together and were unable to move, which impeded myoblast differentiation (see Figure 4-15).

Other studies have reported this shrinkage in collagen hydrogels. Oh et al. seeded rat bone marrow mesenchymal stem cells (MSCs) in collagen hydrogels at different cell seeding densities (from $5 \times 10^{4}$ to $5 \times 10^{5}$ cells $/ \mathrm{mL}$ ) and reported hydrogel shrinkage at 3-4 weeks of cell culture for the hydrogel with the highest cell seeding [233]. Zhang et al. seeded 2, 10, and 50 million cells $/ \mathrm{mL}$ (rabbit bone marrow MSCs) in Type II collagen hydrogels $(7 \mathrm{mg} / \mathrm{mL}$ ). After 7 days of culture $50-$ $60 \%$ hydrogel contraction was obtained for the highest cell seeding density and slightly less at the other two cell densities. They also reported a plateau after cell proliferation at 7 days [57]. Gelatin hydrogel shrinkage thus seems to be related to the cell seeding density of the hydrogels and the strong forces that the cells exert on the surrounding matrix. 
Actin : DAPI
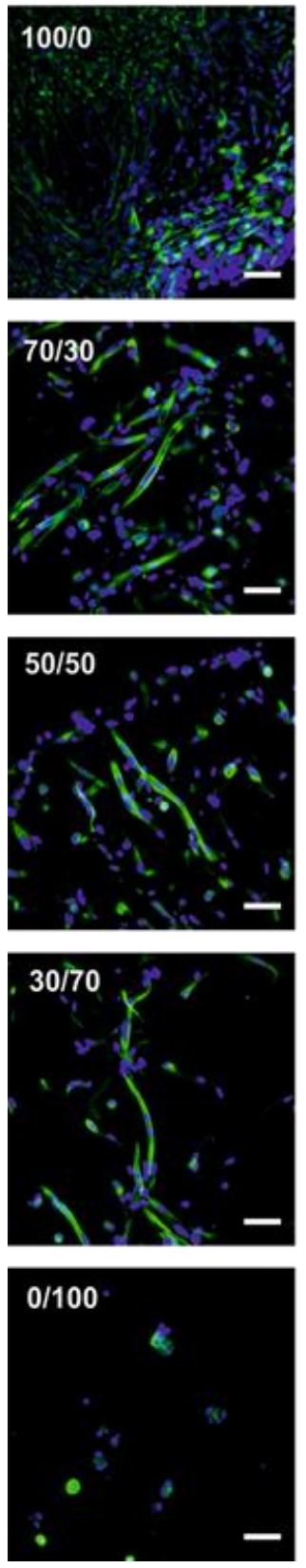

Myosin
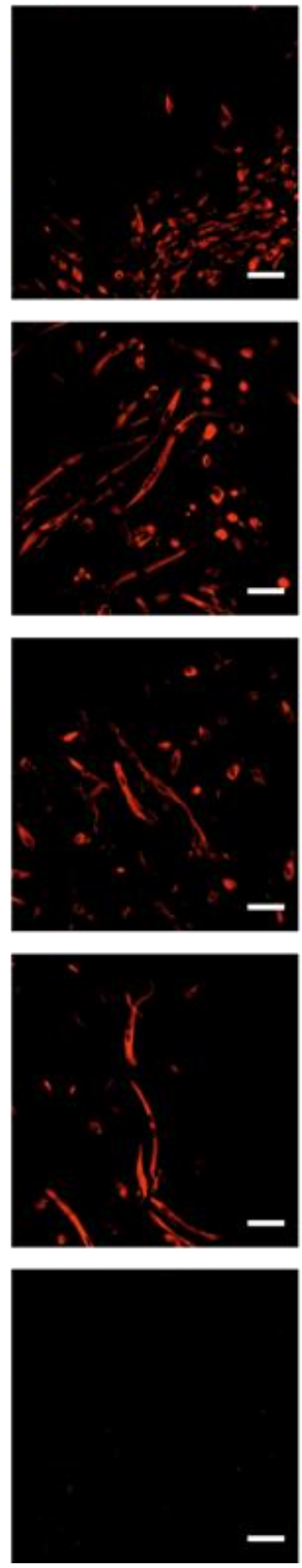

Actin : Myosin : DAPI
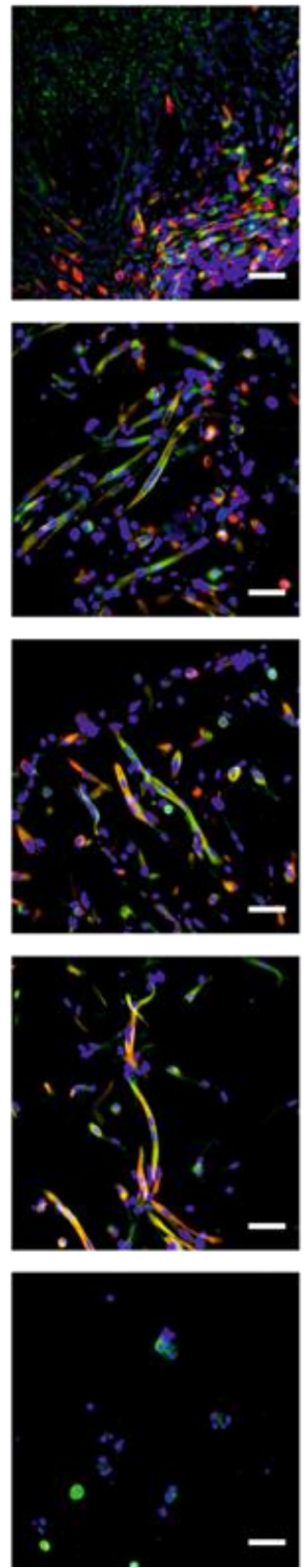

Figure 4-15. Differentiation of $\mathrm{C} 2 \mathrm{C} 12$ cells in the different Gel/HA compositions after 4 days in differentiation media. Myosin, actin and DAPI were stained in $40 \mu \mathrm{m}$ hydrogel sections. Scale bar $50 \mu m$. 
Table 4-6. Myoblast differentiation of the different Gel/HA compositions.

\begin{tabular}{ccc}
\hline \multirow{2}{*}{$\begin{array}{c}\text { Gel/HA } \\
\text { Hydrogel }\end{array}$} & \multicolumn{2}{c}{ Differentiation (\%) } \\
\cline { 2 - 3 } & Periphery & Center \\
\hline $100 / 0$ & $48.8 \pm 8.1$ & $0 \pm 0$ \\
$70 / 30$ & $57.2 \pm 5.2$ & $35.3 \pm 7.6$ \\
$50 / 50$ & $54.5 \pm 8.9$ & $10.8 \pm 5.7$ \\
$30 / 70$ & $57.8 \pm 5.5$ & $26.9 \pm 13.6$ \\
$0 / 100$ & $0 \pm 0$ & $0 \pm 0$ \\
\hline
\end{tabular}

In Gel/HA hybrids, myotube formation could be seen throughout the entire hydrogel, although myoblast differentiation was higher at the periphery (see Table 4-6). It is therefore possible that HA acts as a stabilizer or spacer, i.e. the presence of non-adhesive HA sequences creates zones in which the cells could not attach, diminishing cellmatrix traction forces and thus eliminating hydrogel shrinkage. In comparison, $35 \%$ differentiation was obtained in 70/30 Gel/HA, $11 \%$ in $50 / 50$ and $27 \%$ in $30 / 70$ at the center of the hydrogel, while there was no differentiation at the center in pure gelatin and pure hyaluronic acid. Higher peripheral differentiation was obtained in Gel/HA hybrids ( $58 \%$ for $30 / 70 \mathrm{Gel} / \mathrm{HA}$ ) than in pure gelatin ( $49 \%$ for 100/0 Gel/HA).

\subsubsection{Effect of a cell contractility inhibitor}

At the cell seeding density used $\left(8 \times 10^{6}\right.$ cells $\left./ \mathrm{mL}\right)$ C2C12 cells considerably contracted the gelatin matrix and a very small hydrogel remained after the cell culture. Although it is known that the contractile mechanism must be activated for $\mathrm{C} 2 \mathrm{C} 12$ differentiation into myoblasts [234], [235], this needs to be counterbalanced by the ECM's mechanical properties to be effective. As we wanted to test 
whether the gel shrinkage was actually a consequence of cell contractility, we studied differentiation using Blebbistatin cell contractility inhibitor in the hydrogels with higher gelatin content (100/0 and 70/30 Gel/HA).

Figure 4-16b shows the contractility effect of the cells on the gelatin matrix. A higher level of hydrogel contraction was obtained in the pure gelatin hydrogel than in $70 / 30 \mathrm{Gel} / \mathrm{HA}$, due to the higher gelatin content and the fact that myoblasts have fewer adhesion sequences. Moreover, the presence of Blebbistatin in the cell culture medium reduced hydrogel contraction in both types. After 4 days, 100/0 Gel/HA had shrunk $63 \%$ in a normal differentiation medium, whereas shrinkage was $48 \%$ in the sample cultured with Blebbistatin. In the case of $70 / 30 \mathrm{Gel} / \mathrm{HA}$ the shrinkage was much less marked, $27 \%$ in the differentiation medium and $13 \%$ with the contractility inhibitor. Both 100/0 and 70/30 Gel/HA compositions show less shrinkage when the culture was made with the cell contractility inhibitor, which proves the effect of cell contractility on the gelatin matrix. No real differences were obtained when the percentage of differentiation was analyzed. Immunofluorescence images of 100/0 and 70/30 $\mathrm{Gel} / \mathrm{HA}$ cultured in the presence of Blebbistatin can be seen in Figure $4-16 a$.

\subsubsection{Discussion}

No shrinkage was observed in HA hydrogels at the cell seeding density used in this study. Toh et al. used a seeding density of $1 \times 10^{7}$ cells $/ \mathrm{mL}$ in HA hydrogels with different crosslinking densities and reported a reduced hydrogel diameter with the reduced cross-linking grade. Hydrogel contraction started by day 6 of culture for the least crosslinked and after 12 days for the most crosslinked hydrogel [111]. 
a)
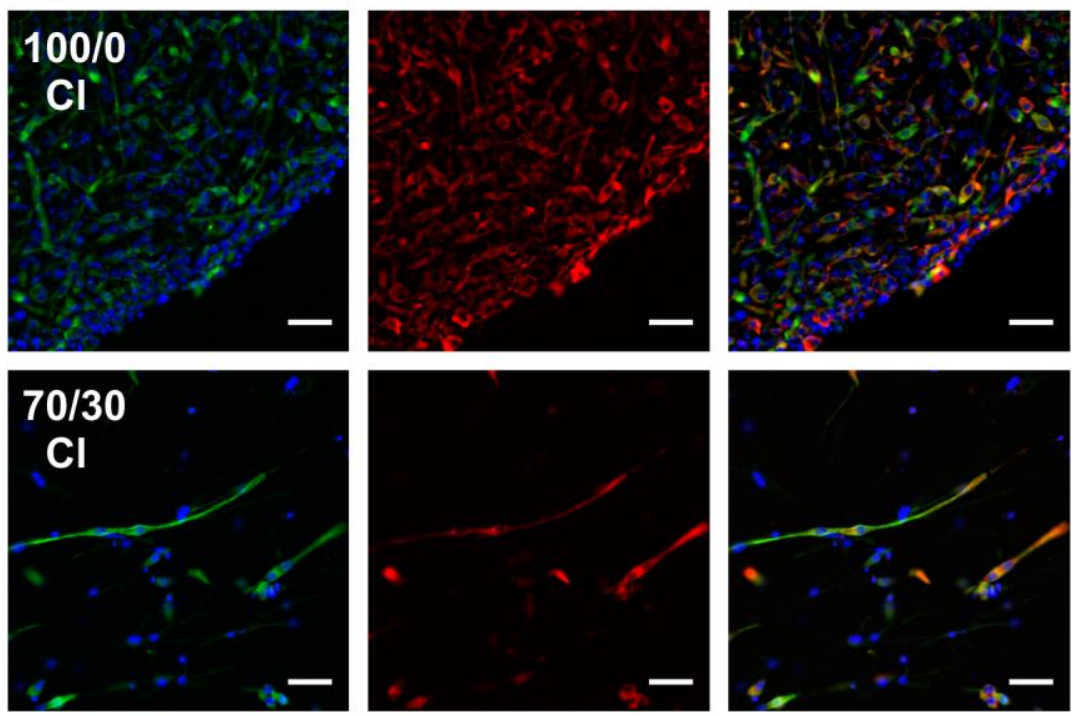

Actin:Myosin:DAPI

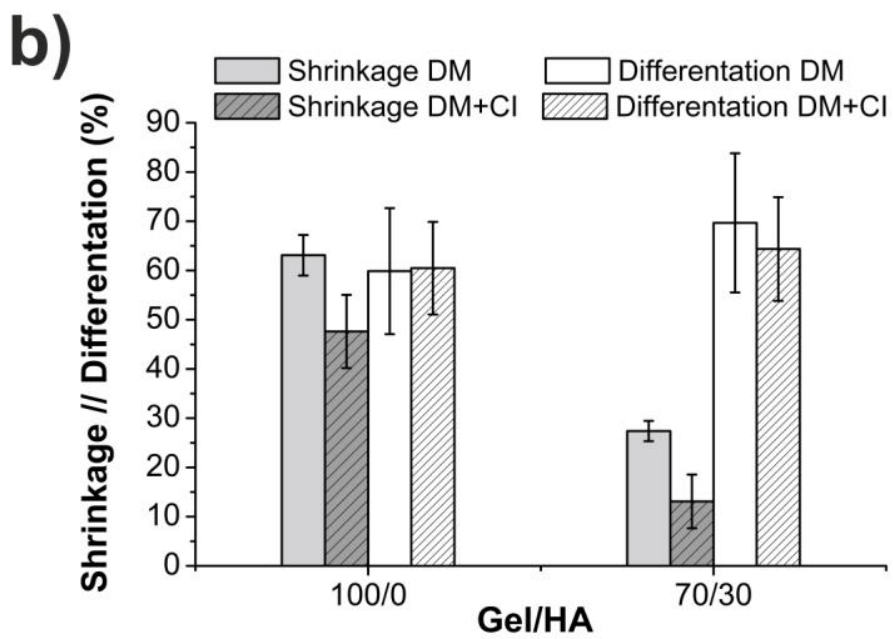

Figure 4-16. a) C2C12 differentiation in 100/0 and 70/30 Gel/HA hydrogels in the presence of Blebbistatin (a contractility inhibitor, $\mathrm{Cl}$ ). b) Shrinkage and percentage of differentiation for 100/0 Gel/HA and $70 / 30 \mathrm{Gel} / \mathrm{HA}$ in differentiation media (DM) and in medium with Blebbistatin $(\mathrm{DM}+\mathrm{Cl})$ after 4 days of culture. Scale bar is $50 \mu \mathrm{m}$. 
Gel/HA hydrogels that contained both gelatin and hyaluronic acid in their structure overcame the gelatin shrinkage problem, allowing myotube differentiation at the center of the hydrogel. The combination of Gel/HA at different ratios, as has been mentioned above, has an influence on: $i$ ) the quantity of RGD sequences in relation to the quantity of gelatin, ii) the quantity of water in the hydrogel (higher HA content increases water retention), iii) the mechanical properties (higher Young's modulus and shear loss modulus are obtained with the higher $\mathrm{HA}$ ) and iv) the ability of cells to upregulate contractility.

The presence of RGD sequences in Gel/HA hydrogels improves the cell adhesion required for myoblast migration, adhesion and myotube formation. Hyaluronic acid is a polysaccharide without the RGD sequence and with an anionic charge that does not favor cellmatrix interaction, since cell surfaces are also anionic [69] and this was the reason why no differentiation in pure hyaluronic acid was obtained. On the other hand, when $8 \times 10^{6} \mathrm{C} 2 \mathrm{C} 12$ cells $/ \mathrm{mL}$ were seeded in pure gelatin, the high amount of adhesion sites enhanced cellular proliferation, increasing the traction forces exerted by the cells on the gelatin network and causing the hydrogels to shrink.

High water content usually reduces cell adhesion [130]. The hydrogels studied in the present work had high water content, with EWC ranging from $3000 \%$ for Gel to $8800 \%$ for HA (see EWC in Table 4-3). EWC is influenced mainly by $H A$, and water content increases with HA content. Compared to Gel/HA hybrids, the results obtained indicate that there is no correlation between the differentiation percentage and water content, since no differences were obtained between differentiations at the periphery and no trend was observed between differentiations at the hydrogel center. For $30 / 70 \mathrm{Gel} / \mathrm{HA}$ hydrogel $27 \%$ differentiation was obtained at the center compared to $11 \%$ differentiation in 50/50. Since there are more RGD adhesion sequences and less water in 50/50 hydrogels, the latter could be expected to have higher cell differentiation, since the water content 
in all the hydrogels is already very high and does not cause any differences in cell differentiation.

Pure hyaluronic acid hydrogel has a Young's Modulus in the same order of magnitude of soft tissues [236] but has no cell adhesion sequences; to solve this, gelatin has been incorporated into its structure to modify its mechanical properties. Changes in the mechanical characteristics of the polymeric network can influence the cell traction forces exerted on it, as it is easier for cells to contract weaker matrices. Gelatin is weaker than a hyaluronic acid hydrogel (compression modulus of $4 \mathrm{kPa}$ for Gel versus $7 \mathrm{kPa}$ for $\mathrm{HA}$ ) and intermediate values were obtained in the hybrids (see Table 4-3). Gelatin's low modulus makes it easy for cells to contract its matrix, makes the hydrogel shrink and makes the material unstable, thus precluding its use as an implant or scaffold. The highest Young's Modulus obtained in the Gel/HA hybrids was for 30/70 Gel/HA with $5.5 \mathrm{kPa}$.

\subsubsection{Conclusions}

A series of Gel/HA (100/0, 70/30, 50/50, 30/70 and 0/100 v/v) injectable hydrogels were synthesized and the proportions of both components were varied in order to study the effects on $\mathrm{C} 2 \mathrm{C} 12$ cell behaviour. As we had hypothesized, $\mathrm{C} 2 \mathrm{C} 12$ cells adhere better to pure gelatin hydrogels due to RGD adhesion sequences. The good cell adhesion of this type of hydrogel and the traction forces that the cells exert on the gelatin matrix cause significant hydrogel shrinkage. Regarding myoblast differentiation into myotubes, no differentiation was achieved in pure hyaluronic acid hydrogels due to the lack of cell adhesion to the matrix. The lack of differentiation at the center of pure gelatin was attributed to the high hydrogel shrinkage. For all the other Gel/HA combinations, when both gelatin and hyaluronic acid were present, myotube formation was achieved throughout the entire hydrogel and no shrinkage occurred, which indicates that this system is a good candidate for further differentiation studies with cells of interest for cartilage tissue engineering. Our last step with 
these mixtures is described in the following section where we encapsulated mesenchymal stem cells and observed their potential for cartilage regeneration. 


\subsection{Chondrogenic differentiation of BM- hMSCs in gelatin/hyaluronic acid injectable hydrogels}

\subsubsection{Materials and Methods}

\subsubsection{Materials}

All the reagents needed for the synthesis of Gel/HA injectable hydrogels with low molecular weight HA (LMW HA) were detailed in 4.3.1.

For cell culture experiments, the human bone marrow human mesenchymal stem cells (BM-hMSCs) were from a commercial lineage (Promocell, Germany). Primary antibodies aggrecan, RUNX2, STRO1 and LPL were purchased from Santa Cruz Biotechnologies (USA). Mouse primary antibody for myosin (MF-20b, $800 \mu \mathrm{g} / \mathrm{mL}$ ) was from Developmental Studies Hybridoma Bank (DSHB, USA). Secondary antibodies rabbit antimouse IgG Cy3 and goat antirabbit IgG Cy3 were obtained from Jackson Immunoresearch (USA). Fungizone, Insulin-Transferrin-Selenium-X (ITS-X), Phosphate Buffered Saline (PBS), Dulbecco's Phosphate Buffered Saline (DPBS) were from Gibco, ThermoFisher Scientific (USA). BODIPY Phallacidin was from LifeTechnologies (USA). TGF- $\beta 3$ was purchased from R\&D. Mounting medium for cryotomy (OCT Compound) was from VWR (USA). Vectashield with DAPI was obtained from Vector Laboratories (USA). DPX Mounting Medium was from Fisher Scientific (USA). Alcian Blue 8GX and Nuclear Fast Red (94\%, pure) were from Acros Organics (USA). All the other reagents for cell culture experiments were purchased from Sigma-Aldrich. 


\subsubsection{Cell culture in Gel/HA hydrogels (bright field images)}

BM-hMSCs were expanded in the presence of a growth medium (GM) consisting of DMEM high glucose based medium with $0.4 \%$ penicillin/streptomycin solution (stock solution, $10000 \mathrm{U} / \mathrm{mL}$ penicillin and $10 \mathrm{mg} / \mathrm{mL}$ streptomycin), $1 \mathrm{mM}$ L-Glutamine, $0.05 \%$ Fungizone (stock solution at $250 \mu \mathrm{g} / \mathrm{mL}$ ), $100 \mu \mathrm{M}$ sodium pyruvate and $10 \% \mathrm{FBS}$, at $37^{\circ} \mathrm{C}$ and $5 \% \mathrm{CO}_{2}$ in an incubator.

$2 \% \mathrm{w} / \mathrm{v}$ Gel and $2 \% \mathrm{w} / \mathrm{v}$ LMW HA solutions were prepared as explained in 4.4.1.2. BM-hMSCs were then detached from the flask using trypsin EDTA, neutralized with GM, centrifuged at $1400 \mathrm{rpm}$ for 5 min, resuspended in GM and counted with the hemocytometer. The required amount of BM-hMSCs (passage 6-7) cells $\left(1 \times 10^{6}\right.$ cells $/ \mathrm{mL}$ ) was added to each Gel/HA mixture. Finally, $45 \mu \mathrm{L}$ of the $\mathrm{Gel} / \mathrm{HA}$ cell suspension was cross-linked with $5 \mu \mathrm{L}$ of $20 \mathrm{mM} \mathrm{H}_{2} \mathrm{O}_{2}$ on each well of the cell culture plate and left in an incubator at $37{ }^{\circ} \mathrm{C}$ and $5 \% \mathrm{CO}_{2}$ for $30 \mathrm{~min}$ to ensure hydrogel crosslinking. Finally, triplicates of each composition were cultured in $\mathrm{GM}$ and chondrogenic media (CM), the latter composed of GM without FBS and with $100 \mathrm{nM}$ dexamethasone, $1 \%$ ITS-X, $50 \mu \mathrm{g} / \mathrm{mL}$ ascorbic 2-phosphate, $40 \mu \mathrm{g} / \mathrm{mL}$ L-proline and $10 \mathrm{ng} / \mathrm{mL}$ TGF- $\beta 3$. Cell culture was followed for 14 days (optical microscope images were taken at 2, 7 and 14 days). The cell medium was changed every 2 days.

\subsubsection{Live/dead analysis}

The viability of cells in the injectable Gel/HA hydrogels was evaluated of culture using the Live/Dead kit for mammalian cells. After 14 days the samples were washed with DPBS and incubated for $15 \mathrm{~min}$ at $37^{\circ} \mathrm{C}$ in DPBS with $1 \mu \mathrm{M}$ of calcein $\mathrm{AM}$ and $2 \mu \mathrm{M}$ ethidium homodimer-1 (EthD-1). Then, in vivo analysis of live (stained in green with calcein AM) and dead cells (stained in red with EthD-1) was assessed in the fluorescence microscope Zeiss Observer Z1_AX10. Several images were taken from two different replicates of each 
sample. The resulting images were representative of the whole sample.

\subsubsection{Immunofluorescence study}

After 14 days of culture, the samples were washed with PBS, fixed with $4 \%$ formaldehyde for $15 \mathrm{~min}$ and washed again with PBS to remove the formaldehyde solution. After the fixing step, the samples were soaked overnight in $30 \% \mathrm{w} / \mathrm{v}$ sucrose in DPBS, embedded in OCT and frozen with liquid nitrogen. Finally, $40 \mu \mathrm{m}$ sections were cut out with a Leica CM 1860 UV cryostat.

Gel/HA samples cultured in GM and CM were immunostained for aggrecan, a characteristic component of articular cartilage. Firstly, the sections on slides were washed and rehydrated with PBS, permeabilized with $0.1 \%$ Triton X-100 in PBS for $20 \mathrm{~min}$ at room temperature (RT) and given two 5 min washes with PBS. The blocking buffer, formed by $1 \%$ BSA solution in PBS, was then added for $1 \mathrm{~h}$ at RT, and two washes with PBS were performed. Aggrecan primary antibody was incubated at 1:100 in blocking buffer for $1 \mathrm{~h}$ at RT, washed and donkey antirrabbit rhodamine secondary antibody was incubated in blocking buffer for $1 \mathrm{~h}$ at RT. Finally, two washes with PBS of 10 min were carried out; actin was stained with BODIPY FL Phallacidin 1:100 in PBS for 30 min at RT, washed and the slides were mounted in Vectashield with DAPI.

For Gel/HA samples cultured in GM, other antibodies characteristic of other cell lineages were tested to determine whether the BM-hMSCs in these types of gels tend to differentiate into one or other cell lineage. For this, the hydrogels were permeabilized with $0.5 \%$ Triton $\mathrm{X}-100$ in PBS at RT, rinsed with PBS twice for 5 minutes, blocked in $1 \%$ $\mathrm{BSA} / 0.1 \%$ Triton $\mathrm{X}-100$ in PBS for $1 \mathrm{~h}$ at RT and rinsed with PBS. The following primary antibodies were then incubated in blocking buffer for $1 \mathrm{~h}$ at RT: rabbit polyclonal RUNX2, mouse monoclonal STRO-1, rabbit polyclonal LPL and mouse monoclonal MF-20. Two $5 \mathrm{~min}$ washes were performed and secondary antibody Cy3 antimouse or antirabbit was incubated, according to the primary antibody used, at 
1:200 in blocking buffer for $1 \mathrm{~h}$ at RT. After two washes with PBS, actin was stained with BODIPY Phallacidin 1:100 in PBS, washed twice for $10 \mathrm{~min}$ and the stained sections were mounted in Vectashield with DAPI.

\subsubsection{Alcian blue histochemistry}

Gel/HA hydrogels without cells, as controls, and samples cultured for 14 days in GM or CM were stained with Alcian Blue in order to localize glycosaminoglycans (GAGs) within the hydrogels. Samples were fixed with $4 \%$ formaldehyde, as previously described. After the fixing step, the samples were soaked overnight in $30 \% \mathrm{w} / \mathrm{v}$ sucrose in DPBS, embedded in OCT, cut to $40 \mu \mathrm{m}$ with the cryostat and rehydrated by washing with PBS twice for $5 \mathrm{~min}$. The constructs were then incubated in $1 \%$ Alcian Blue in $0.1 \mathrm{~N} \mathrm{HCl}$ at pH 1 for 30 min to stain sulfated GAGs [205], rinsed with tap water, distilled water and counterstained with $0.1 \%$ Nuclear Fast Red for 5 min. The slides with the sections were then rinsed in tap water, a last rinse with distilled water, rehydrated with increasing ethanol solutions (70 and 90\%) and xylene for 1 min each wash. Finally, the sections were mounted with DPX Mounting medium. GAGs positive staining was documented by optical microscopy using brightfield illumination.

\subsubsection{Statistical analysis}

For the statistical studies Statgraphics software was used and the Mann-Whitney (Wilcoxon) test was performed to find any statistical differences between the groups at $95 \%$ confidence intervals $(p<$ $0.05)$.

\subsubsection{Results and discussion}

\subsubsection{1. $B M-h M S C s$ proliferation}

Cell proliferation and morphology were monitored during the cell culture experiment at 2, 7 and 14 days under the microscope (Figure 4-17 and Figure 4-18). Figure 4-17 shows cells in Gel/HA hydrogels 
cultured in a growth medium. In the gelatin hydrogel, the cells adhere and express an elongated morphology from day 2 . In the case of hyaluronic acid and Gel/HA mixtures, the cells remain rounded at day 2 after seeding. In 70/30 and 50/50 hybrids, the cells start to elongate at day 7 , while some long cells could be seen at day 14 in the 30/70 hydrogel. The lack of cell adhesion sites in the pure HA hydrogel $(0 / 100)$ kept the cells rounded during the entire cell culture experiment.

In the cells in Gel/HA hydrogels cultured in a chondrogenic medium (Figure 4-18), the cells proliferated throughout the 14 days of culture, except for the pure HA (0/100). All those cultured in the chondrogenic medium and the hydrogels with gelatin in their composition are larger than those cultured in a growth medium. Furthermore, cell elongation could be seen after 7 days of culture in Gel/HA hybrids. As in the growth medium, the pure hyaluronic acid hydrogel does not allow cell elongation and proliferation due to the lack of cell adhesion sequences.

Cells were alive after the 14 days of culture, see Figure 4-19. 

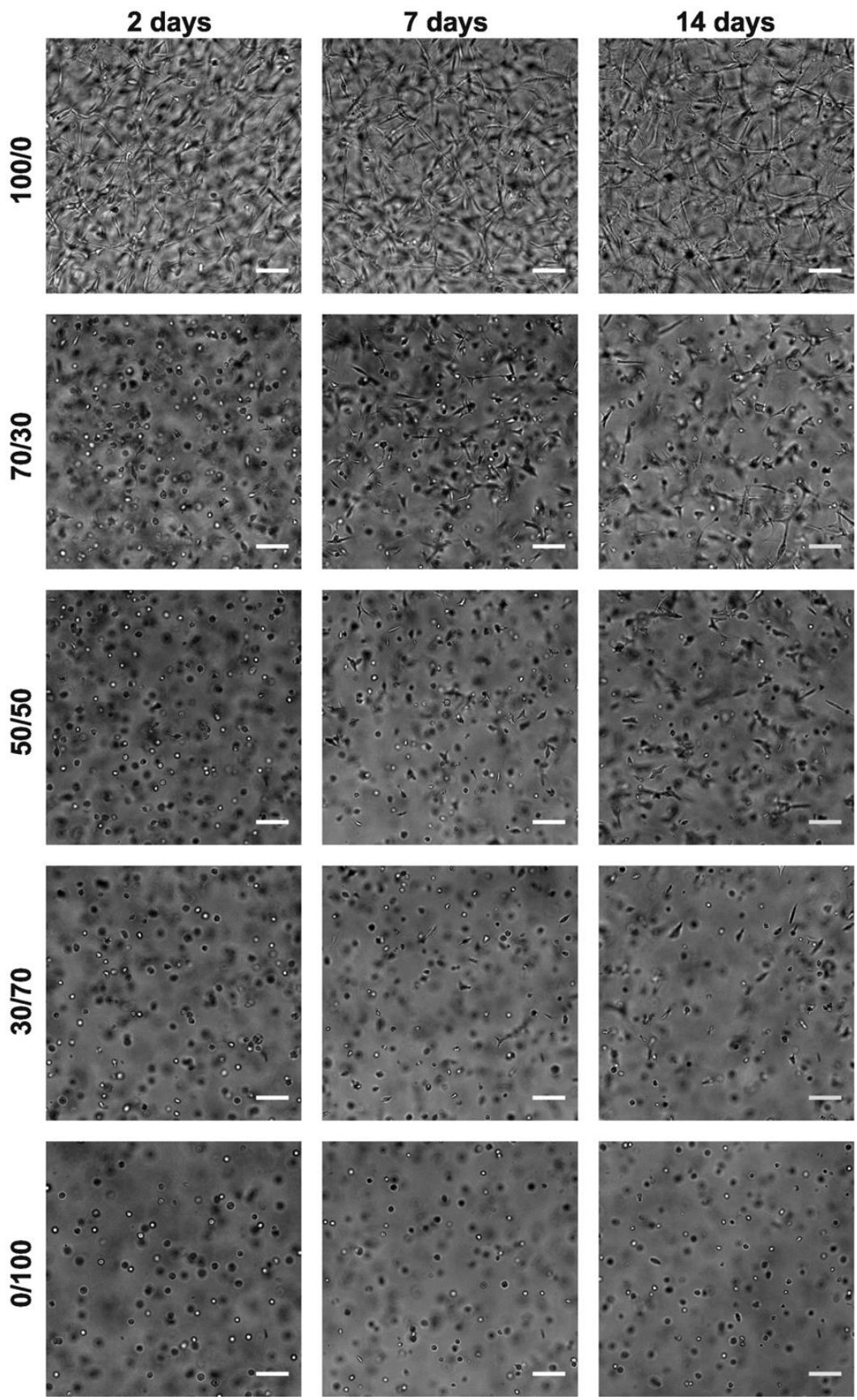

Figure 4-17. Microscope photos of Gel/HA hydrogels cultured in GM for 2, 7 and 14 days. Scale bar $100 \mu m$. 

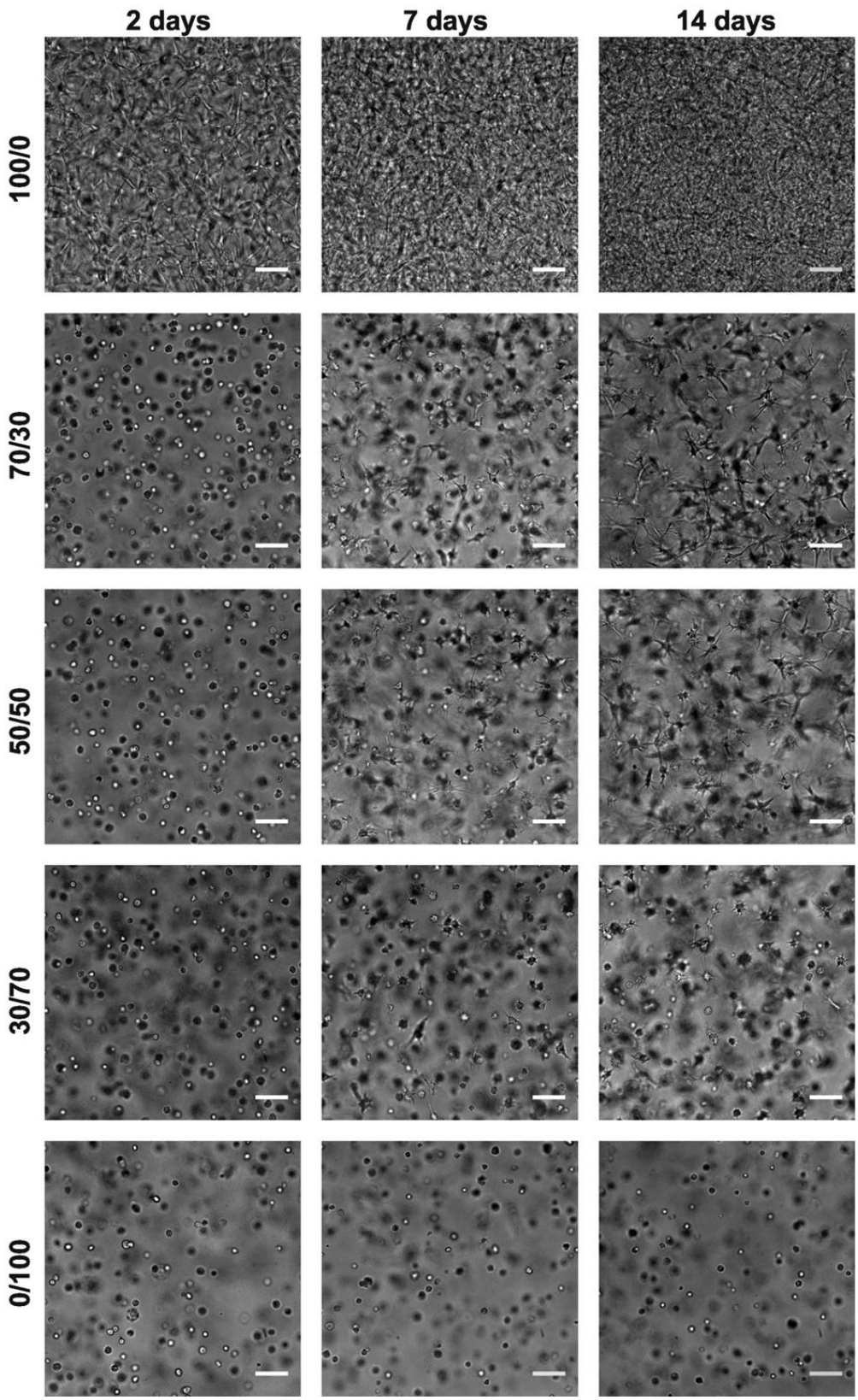

Figure 4-18. Microscope photos of Gel/HA hydrogels cultured in CM for 2, 7 and 14 days. Scale bar $100 \mu \mathrm{m}$. 


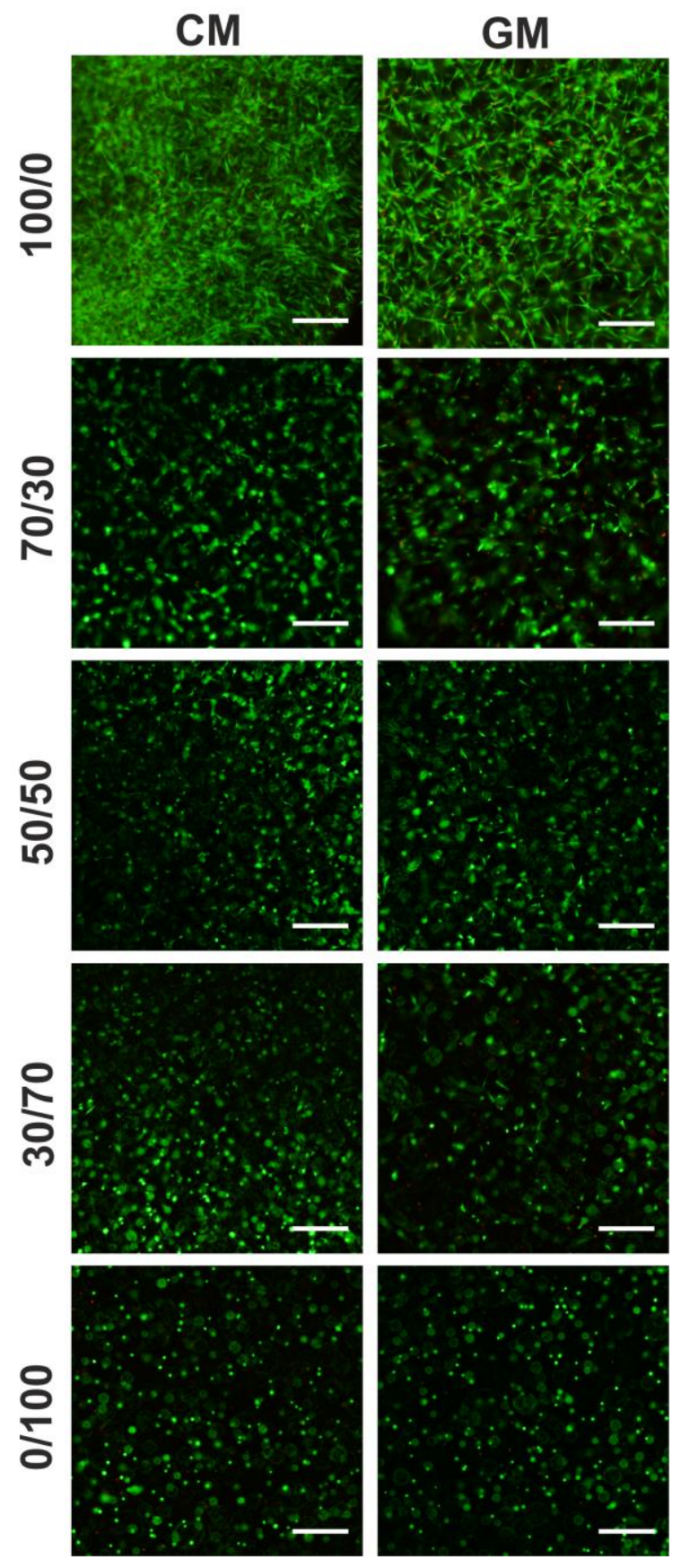

Figure 4-19. Live/dead images of BM-hMSCs cells cultured within Gel/HA hydrogels in GM and CM for 14 days. Scale bar $300 \mu \mathrm{m}$. 


\subsubsection{BM-hMSCs differentiation}

MSCs differentiation towards the different cell lineages (myogenic, adipogenic, osteogenic and chondrogenic) was studied in order to determine whether there was any predilection to differentiate towards any of the lineages when no differentiation medium was used.

First, the presence or absence of stemness after 14 days of culture was studied using STRO1 (Figure 4-20). No signal was obtained for this marker in any of the Gel/HA hydrogels, so that no stemness could be observed in the hydrogels under study after the 14 days of cell culture, even in the cells that remained rounded in the hyaluronic acid hydrogel. The same result was obtained when RUNX2 (osteogenic marker, Figure 4-21), MF20 (myogenic marker, Figure 422) and LPL (adipogenic marker, Figure 4-23) markers were analyzed. No differentiation towards these cell lineages could be seen when these markers were used.

Aggrecan expression was also studied as a typical marker for chondrogenic differentiation (Figure 4-24). In this case, aggrecan was observed in the cells cultured in all Gel/HA compositions. This test was also performed for comparison purposes and as a control with the cells cultured in Gel/HA hydrogels and in CM for 14 days (Figure 4-25). In the case of hMSCs cultured in CM, almost no aggrecan staining was obtained in the pure gelatin hydrogel (100/0), possibly because the cells were still proliferating. 

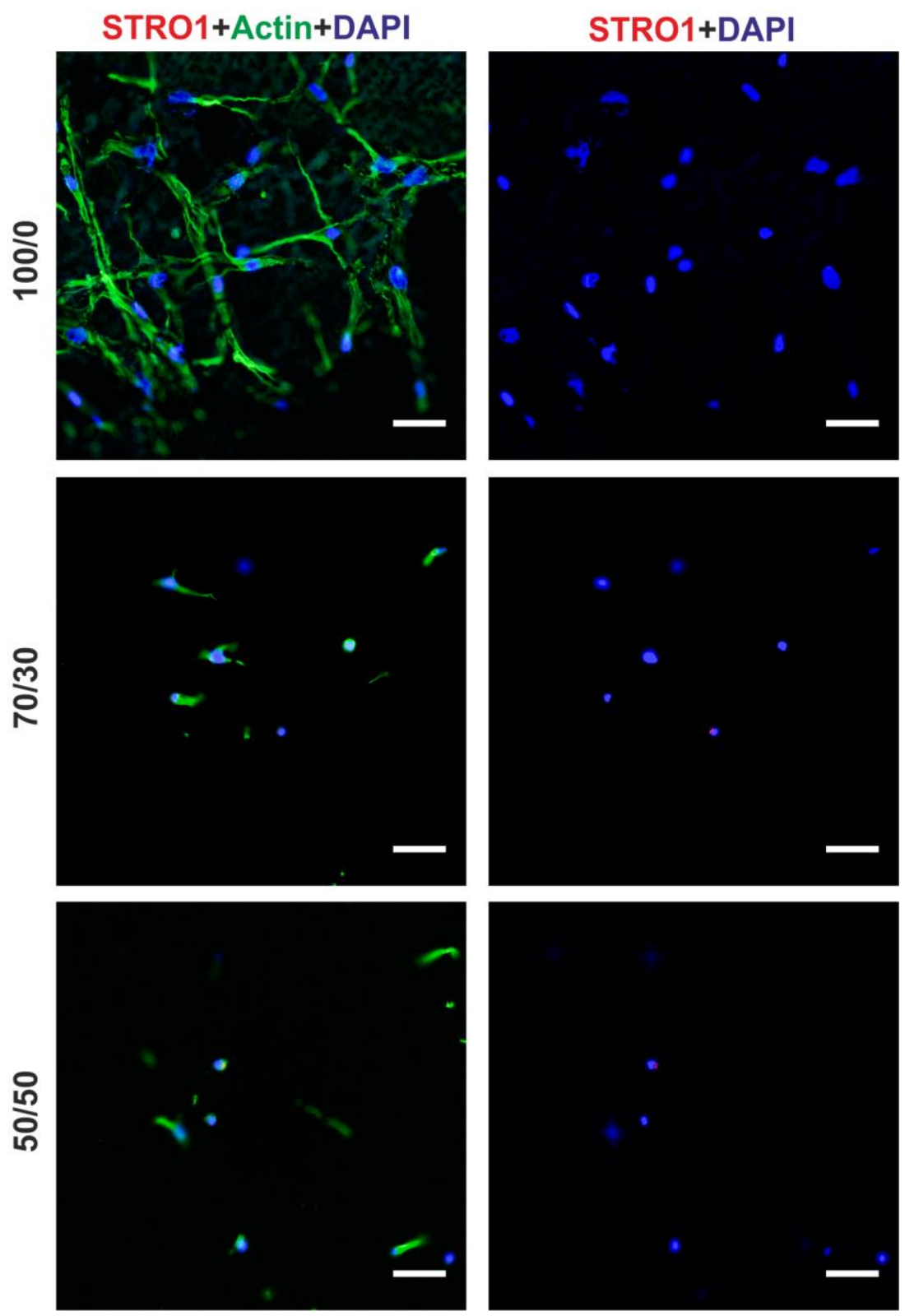

Figure 4-20. (The figure continues in the following page). 


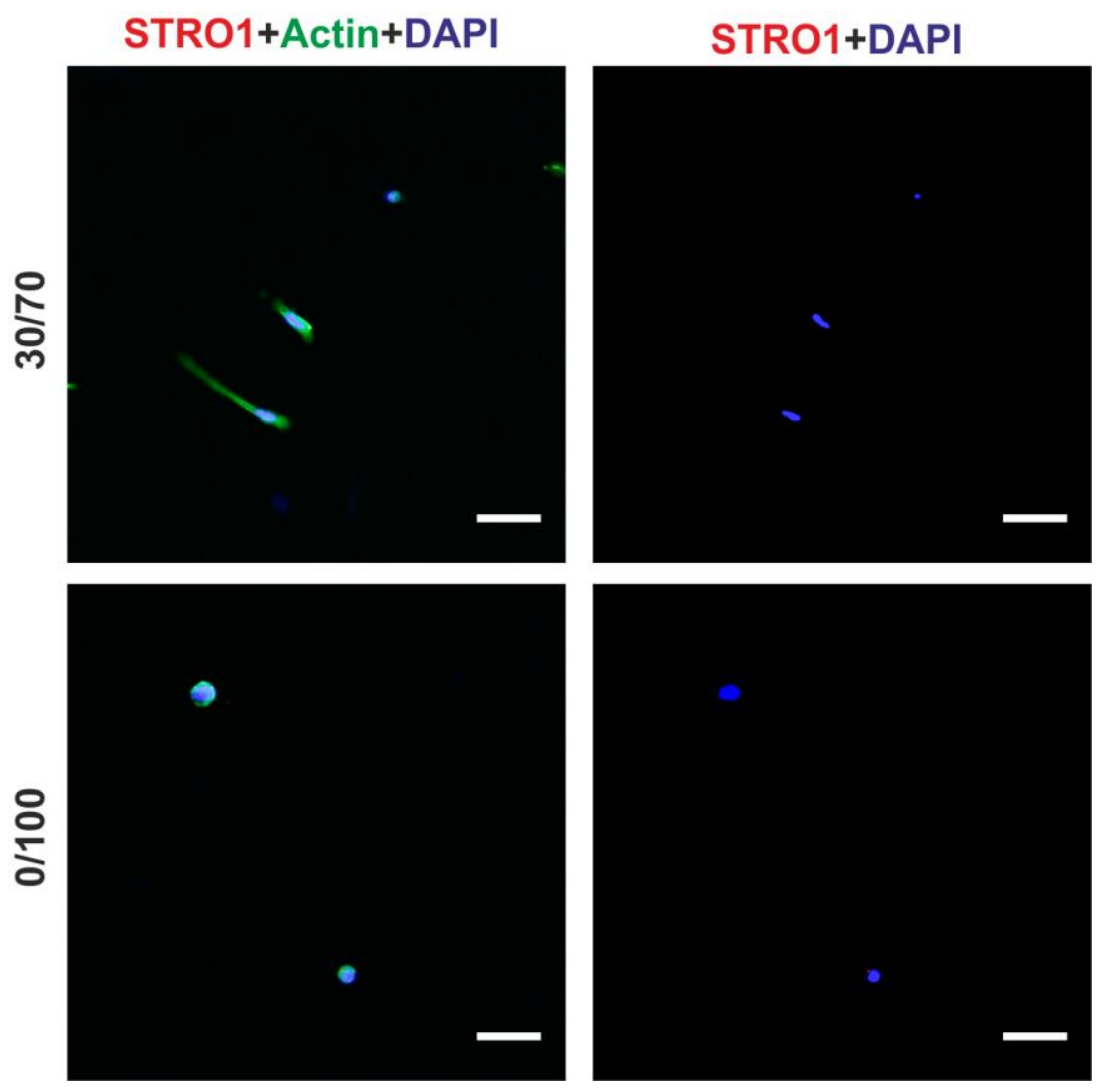

Figure 4-20. Immunofluorescence images for STRO1 of BM-hMSCs cultured in Gel/HA hydrogels and in GM for 14 days. Nuclei are stained with DAPI, cytoskeleton is stained in green and STRO1 is stained in red. Scale bar $50 \mu \mathrm{m}$. 

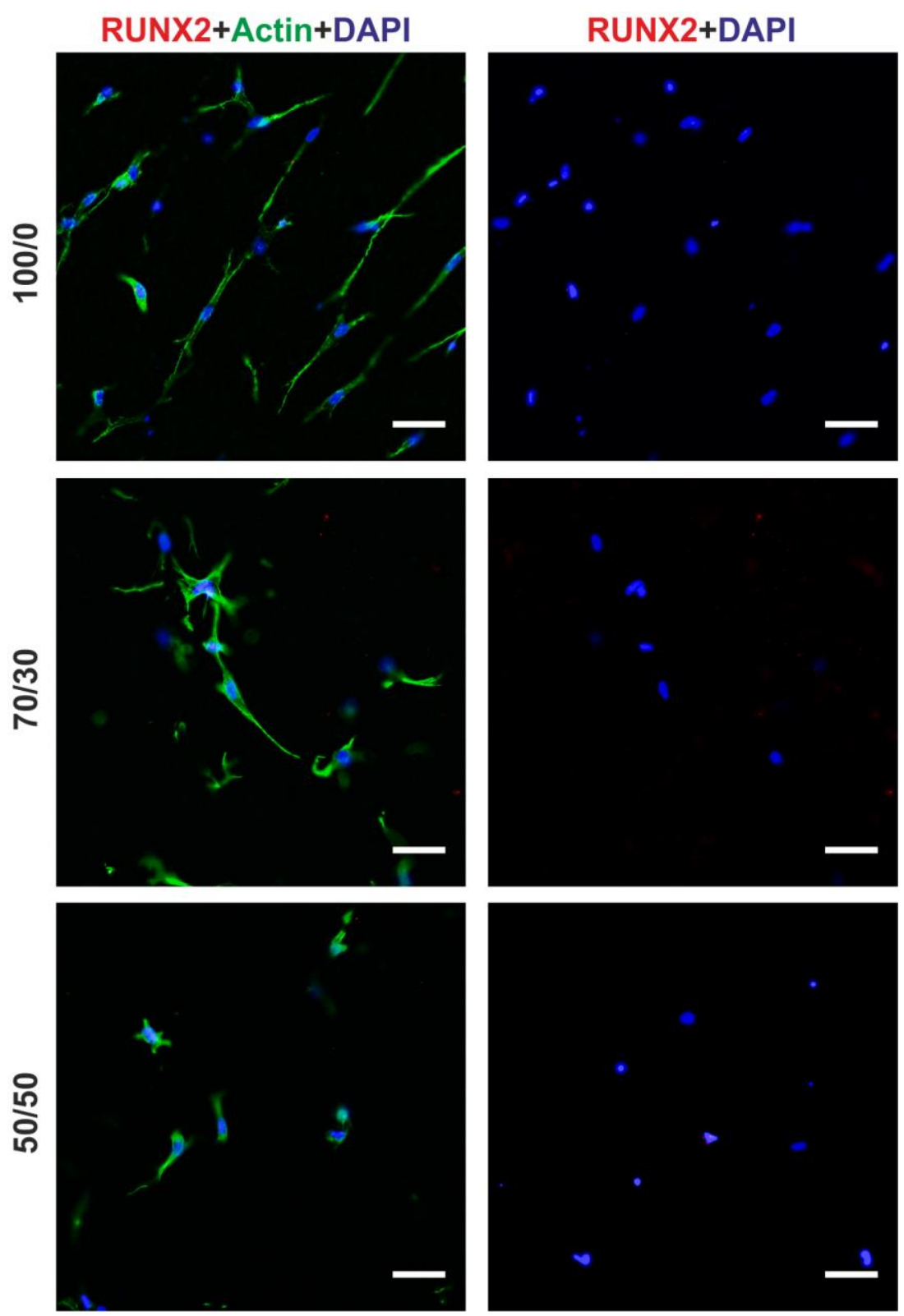

Figure 4-21. (The figure continues in the following page). 


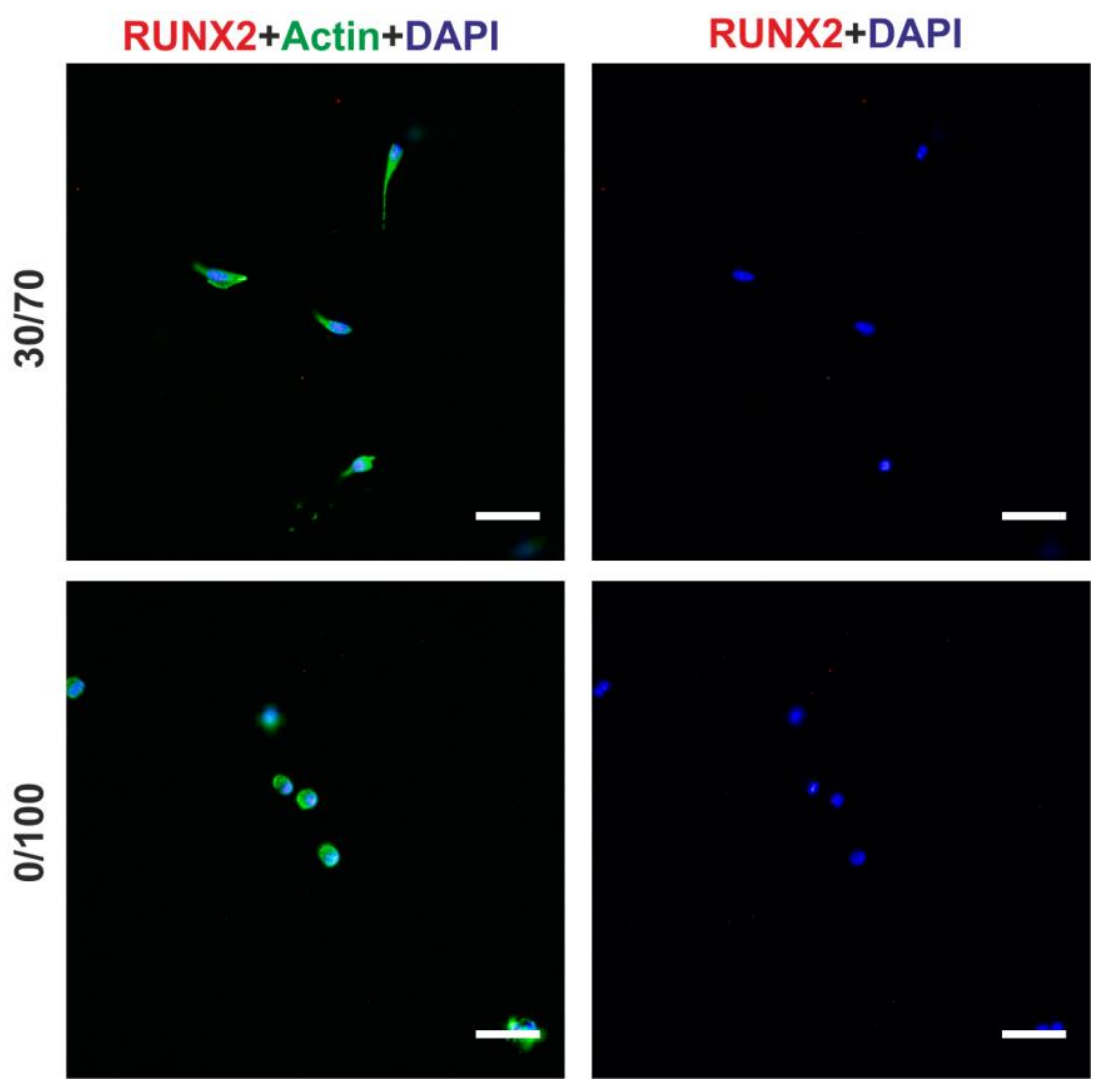

Figure 4-21. Immunofluorescence images for RUNX2 of BM-hMSCs cultured in Gel/HA hydrogels and in GM for 14 days. Nuclei are stained with DAPI, cytoskeleton is stained in green and RUNX2 is stained in red. Scale bar $50 \mu \mathrm{m}$. 


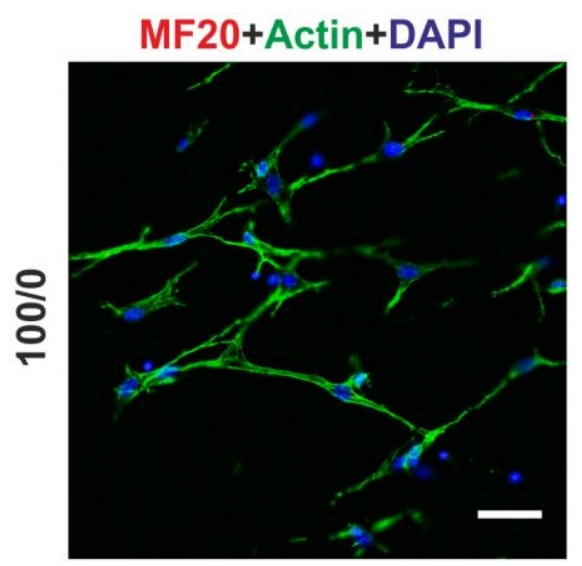

\section{MF20+DAPI}
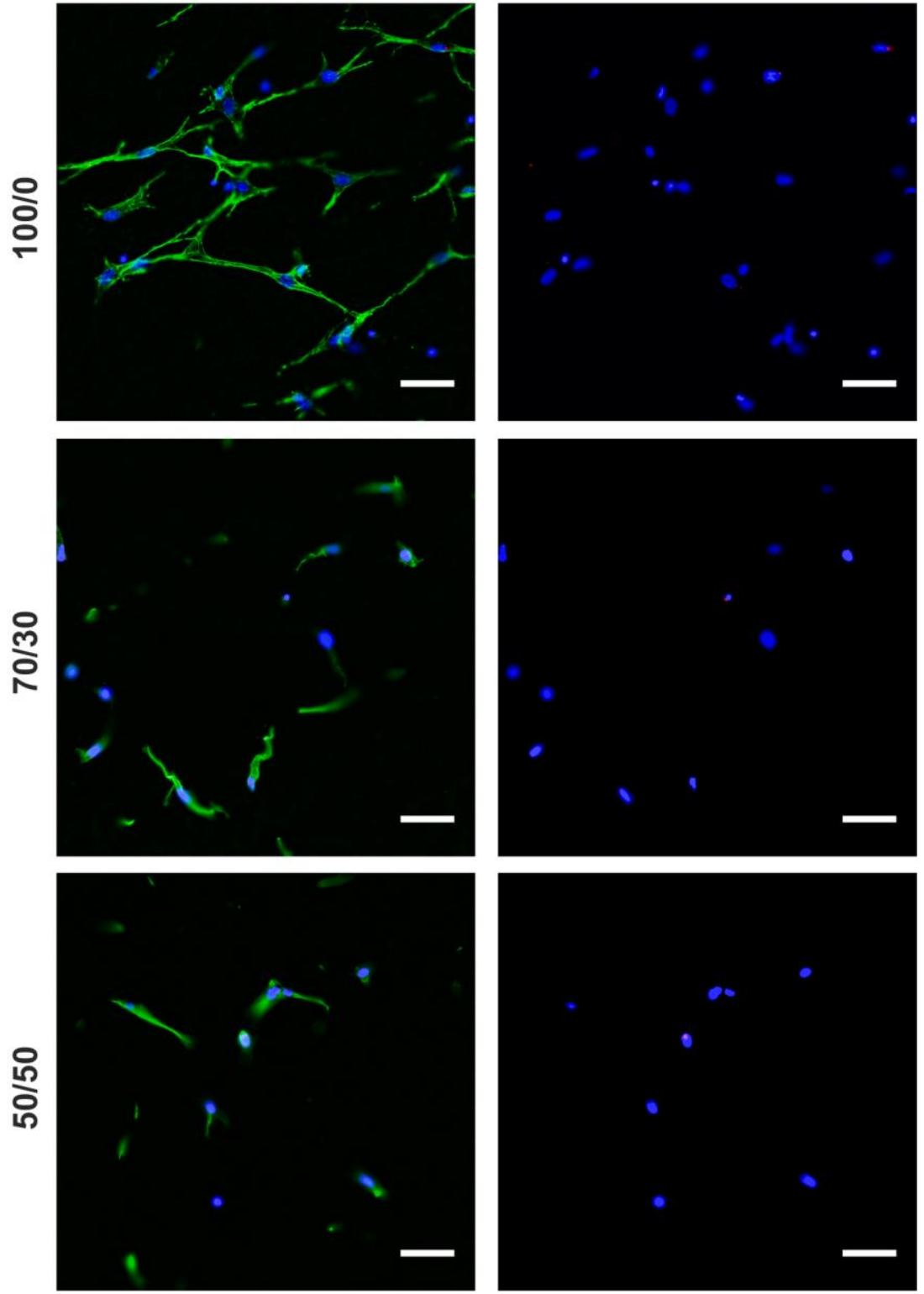

Figure 4-22. (The figure continues in the following page). 


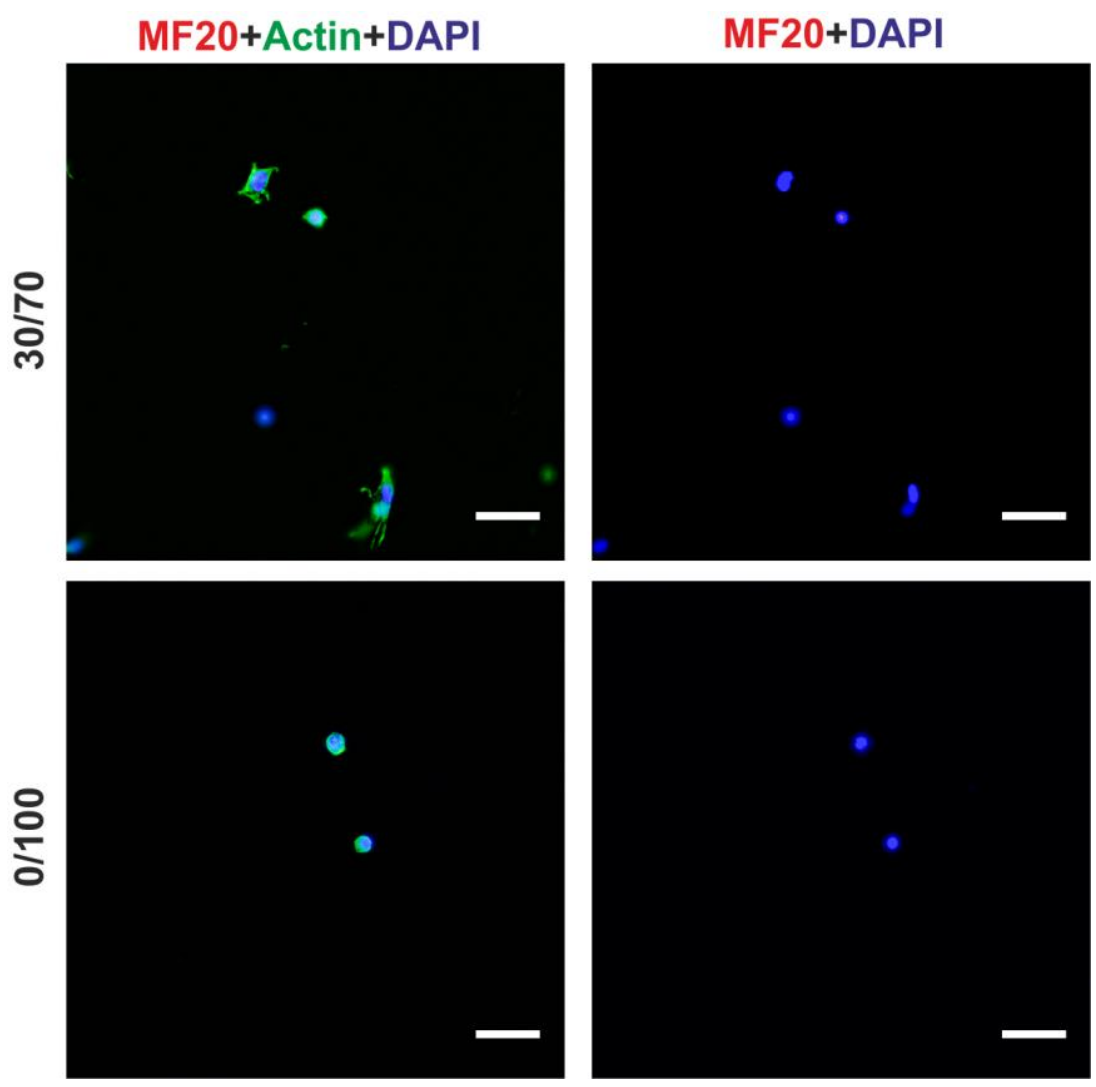

Figure 4-22. Immunofluorescence images for MF20 of BM-hMSCs cultured in Gel/HA hydrogels and in GM for 14 days. Nuclei are stained with DAPI, cytoskeleton is stained in green and MF2O is stained in red. Scale bar $50 \mu \mathrm{m}$. 

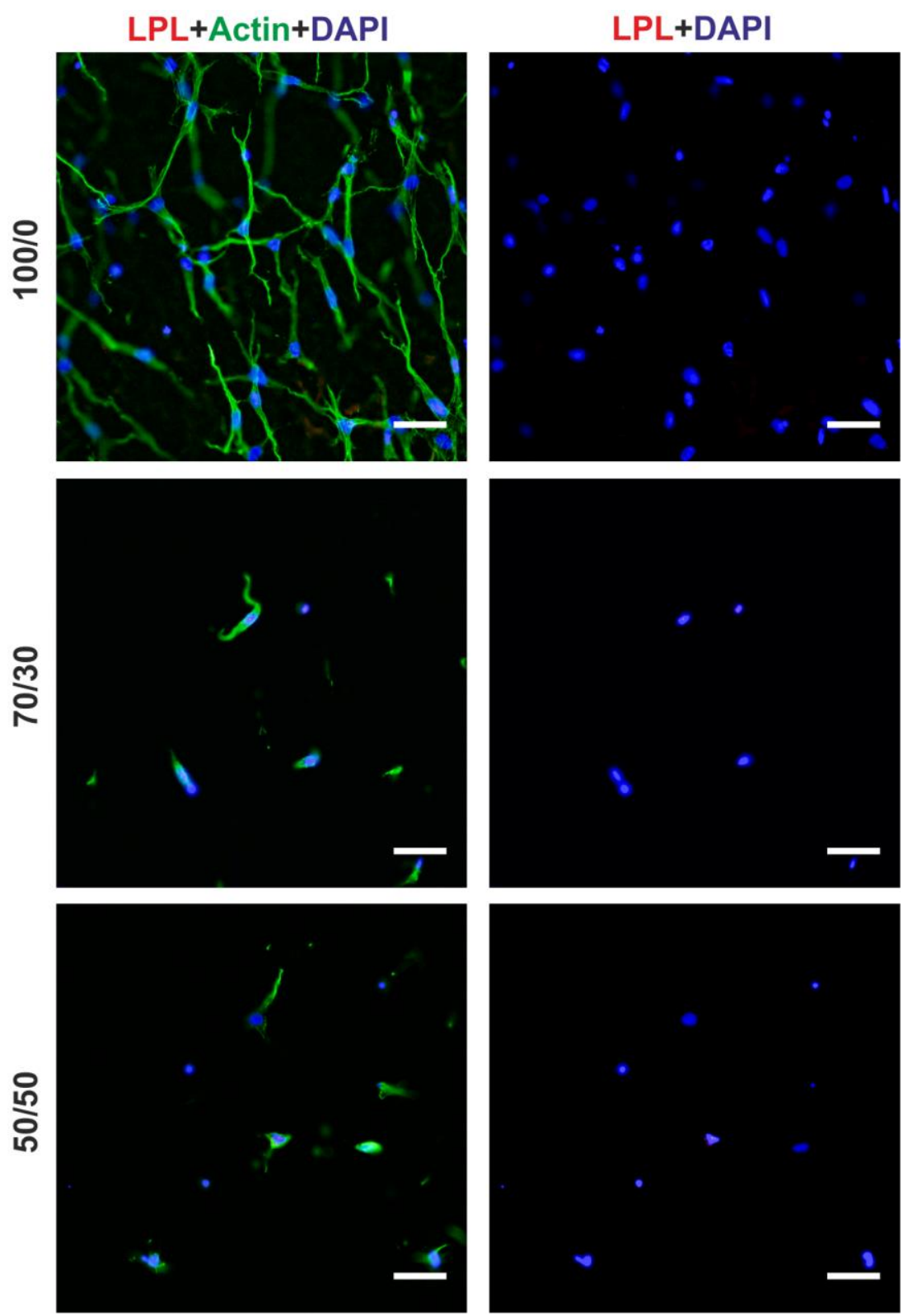

Figure 4.23. (The figure continues in the following page). 

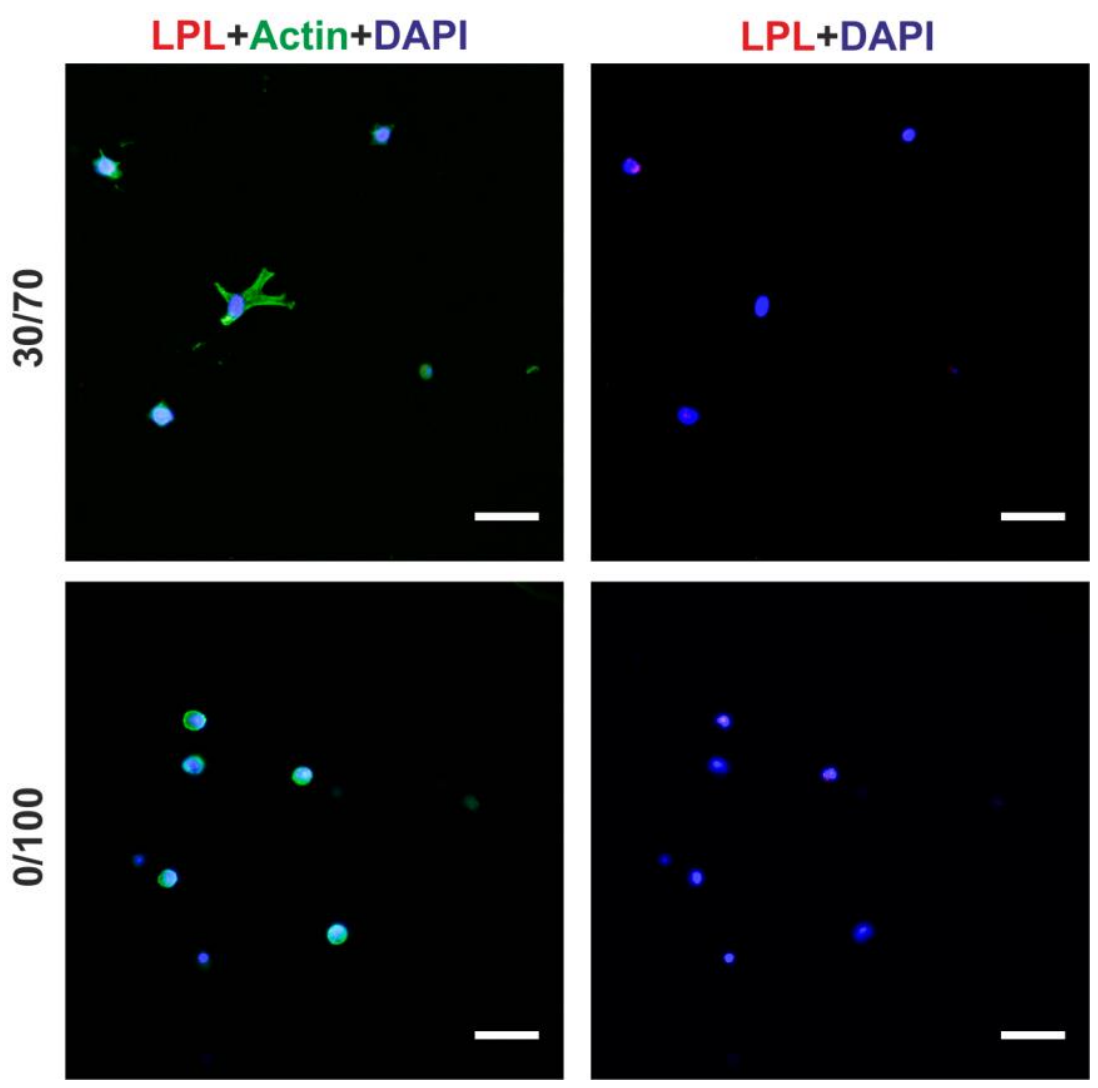

Figure 4-23. Immunofluorescence images for LPL of BM-hMSCs cultured in Gel/HA hydrogels and in GM for 14 days. Nuclei are stained with DAPI, cytoskeleton is stained in green and LPL is stained in red. Scale bar $50 \mu \mathrm{m}$. 

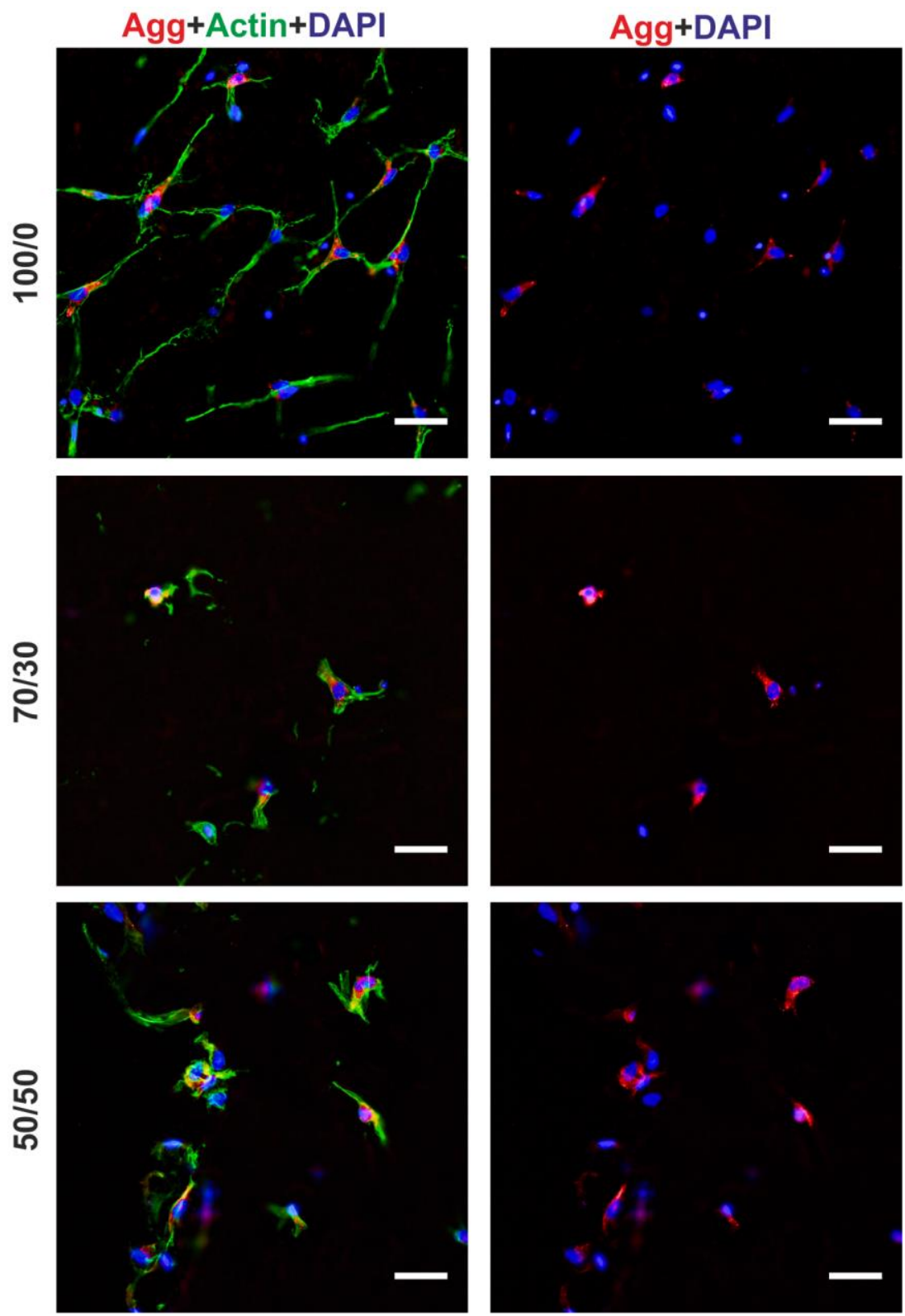

Figure 4-24. (The figure continues in the following page). 


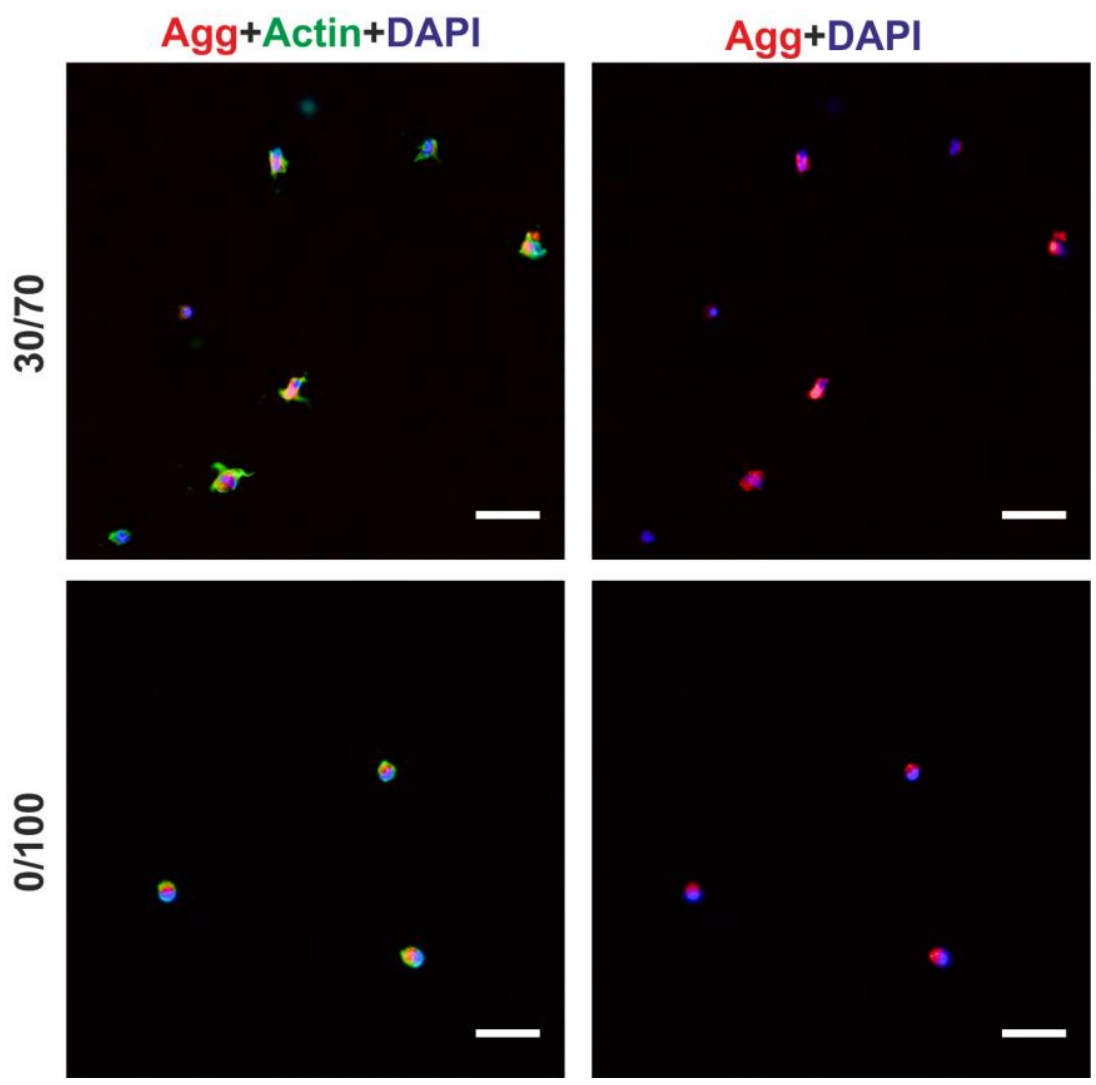

Figure 4-24. Immunofluorescence images for aggrecan (Agg) of BMhMSCs cultured in Gel/HA hydrogels and in GM for 14 days. Nuclei are stained with DAPI, cytoskeleton is stained in green and aggrecan is stained in red. Scale bar $50 \mu \mathrm{m}$. 

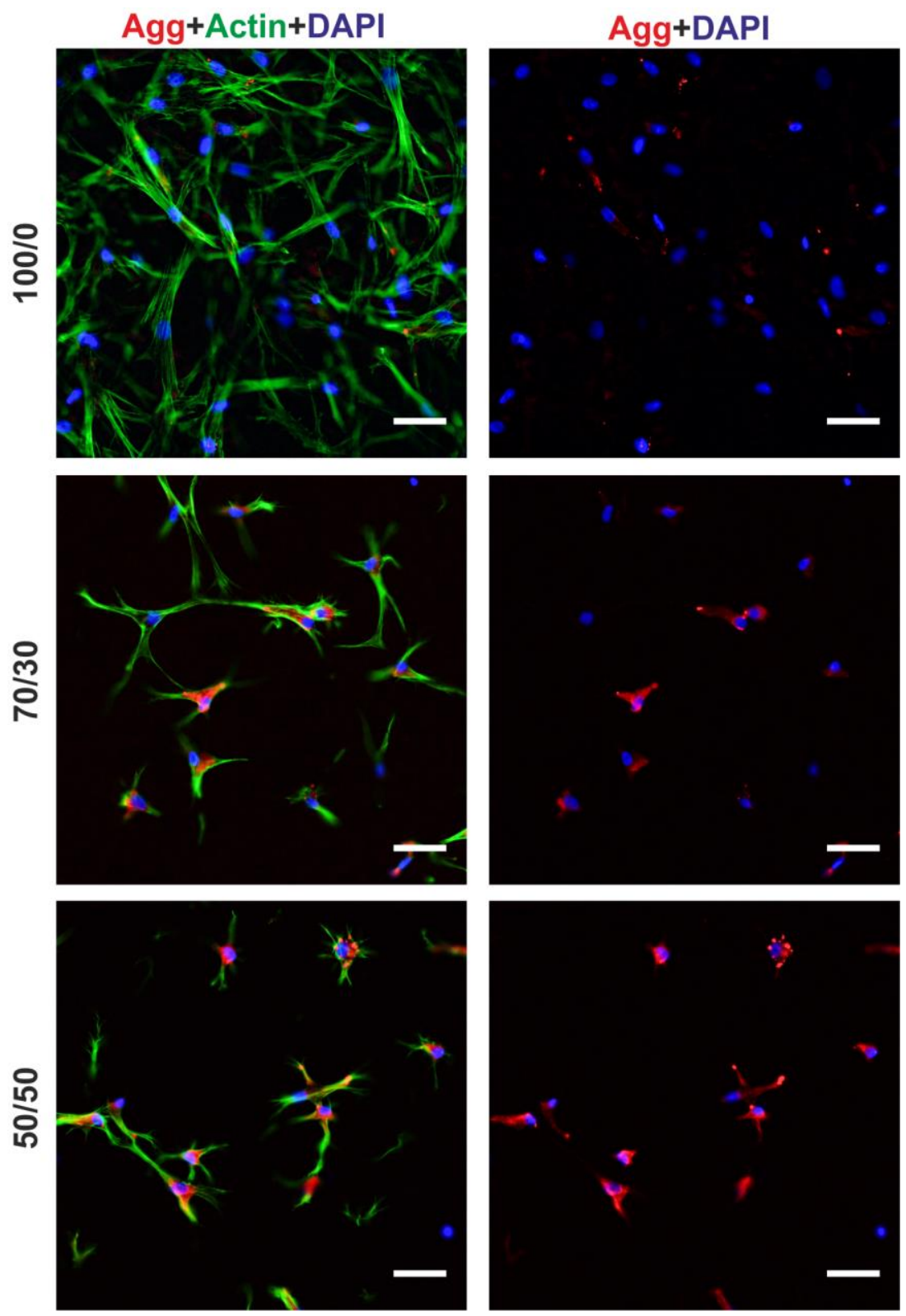

Figure 4-25. (The figure continues in the following page). 

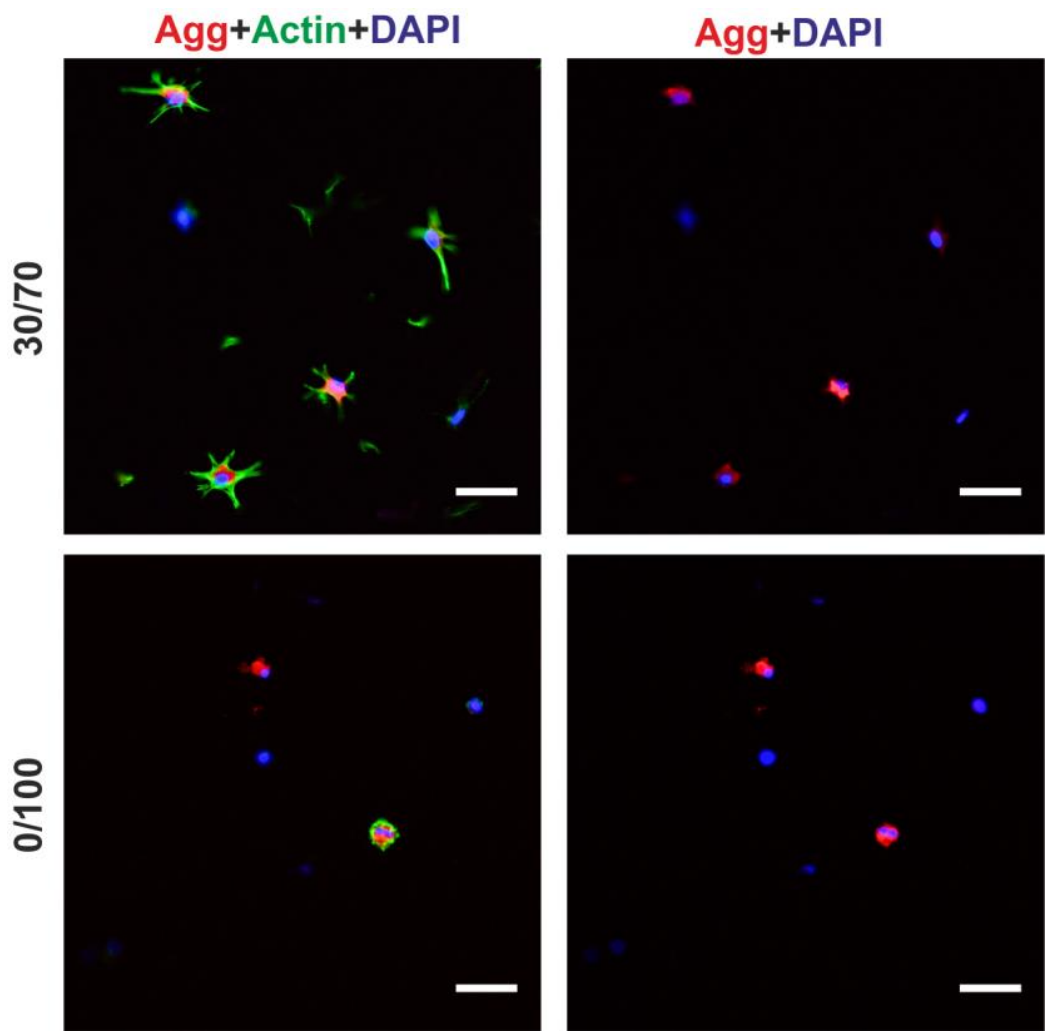

Figure 4-25. Immunofluorescence images for aggrecan (Agg) of BMhMSCs cultured in Gel/HA hydrogels and in CM for 14 days. Nuclei are stained with DAPI, cytoskeleton is stained in green and aggrecan is stained in red. Scale bar $50 \mu \mathrm{m}$. 
From the photos obtained, we calculated the percentage of cells expressing aggrecan (Figure 4-26a) and the number of cells per area (Figure 4-26b). The number of cells per area was seen to increase with the quantity of gelatin in the hydrogel, although there is a marked drop in the number of cells when hyaluronic acid is present in the hydrogel composition. Comparing 100/0 and 70/30 hydrogels, there is a decrease of $66 \%$ for cells in $\mathrm{GM}$ and a $76 \%$ for cells in CM. No significant statistical differences were obtained between the $70 / 30$ and $50 / 50$ hydrogels in either $\mathrm{CM}$ or $\mathrm{GM}$, or between the $50 / 50$ and $30 / 70$ hydrogels cultured in GM.

As regards the percentage of aggrecan expression (Figure 4-26a), the presence of higher amounts of HA in the hydrogel composition increases the percentage of cells expressing aggrecan. In both $\mathrm{CM}$ and GM cultures, statistically significant differences were obtained between all the groups, except between the $50 / 50$ and $30 / 70 \mathrm{Gel} / \mathrm{HA}$ samples. The highest percentage of differentiation was obtained for pure hyaluronic acid hydrogel (0/100) cultured in GM, with $79 \%$. This value was higher than that obtained for the $0 / 100$ sample cultured in $\mathrm{CM}(24 \%)$, although the number of cells in this hydrogel is quite low compared with all the other hydrogel compositions (see Figure 426b). Gel/HA 50/50 and 30/70 hybrids present similar percentages of aggrecan differentiation with a $56 \%$ for CM culture and a $31 \%$ for GM culture. 
a

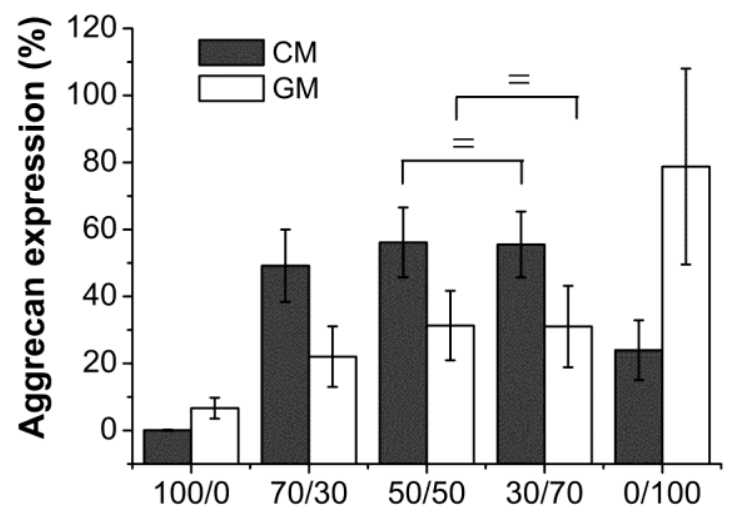

b

Gel/HA

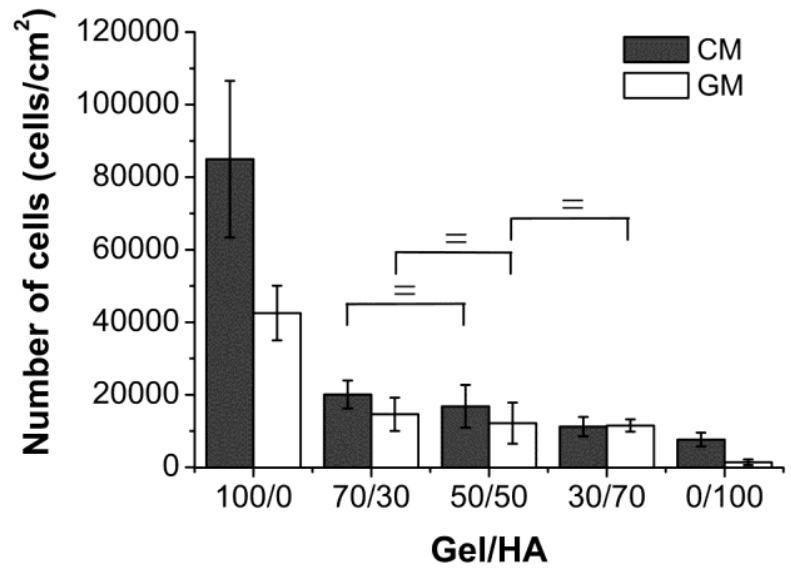

Figure 4-26. a) Aggrecan expression and b) number of cells $/ \mathrm{cm}^{2}$ in $\mathrm{Gel} / \mathrm{HA}$ hydrogels cultured in growth and chondrogenic medium. All groups within a type of culture medium show statistically significant differences between each other, except those marked with $=$.

To further analyze the differentiation towards the chondrogenic lineage of the Gel/HA system, the synthesis of glycosaminoglycans (GAGs) was studied with Alcian Blue. In this histology, GAGs are stained in blue, cells are stained in red and background is pink or becomes purple when the quantity of hyaluronic acid is increased (background of the different hydrogel compositions without cells can 
be seen in Figure 4-27). Figure 4-28a-e shows the presence of GAGs in all the hydrogels cultured in $\mathrm{CM}$. A pale blue color can be seen in the pure gelatin hydrogel (100/0) (Figure 4-28a). For the cells cultured in GM, the images obtained for the 100/0 hydrogel did not contain any blue, so that no GAGs were synthesized by the cells cultured in gelatin hydrogels under this condition. In the case of hMSCs cultured in the hybrids, some cells expressing GAGs were detected by the presence of a blue area around them (white arrows in Figure 4-28g-i). In Figure 4-28j, rounded hMSCs cultured in 0/100 hydrogel can be seen surrounded by a blue layer, indicating the presence of synthesized GAGs.

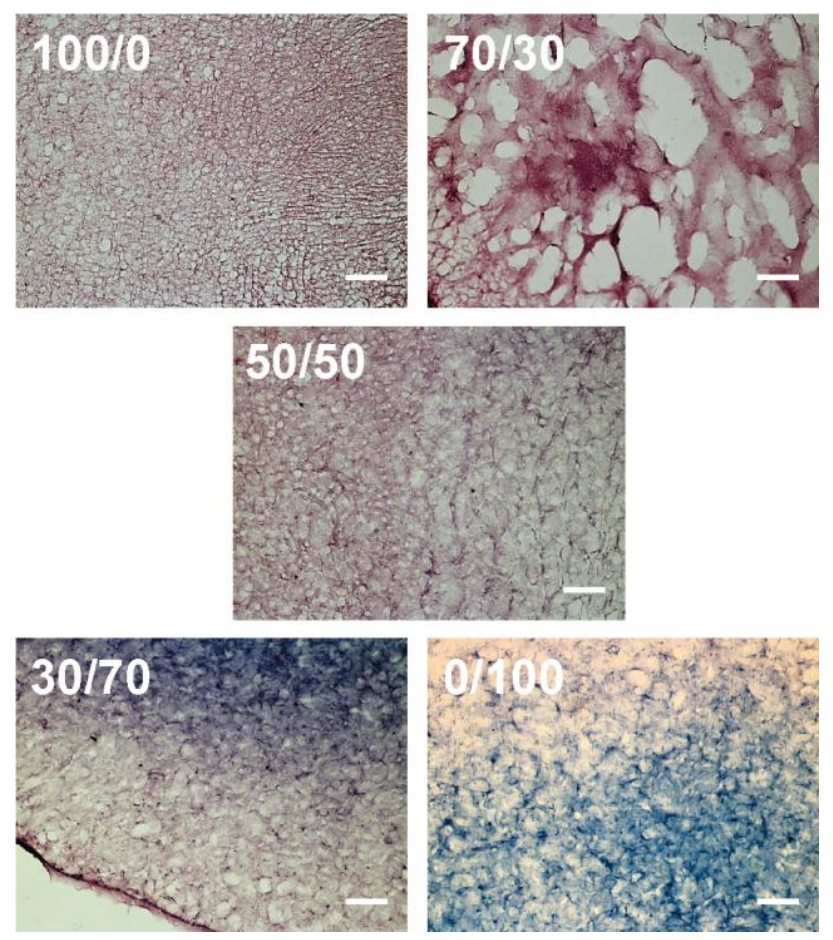

Figure 4-27. Alcian blue assay for Gel/HA (100/0, 70/30, 50/50, 30/70 and 0/100) controls without cells. Scale bar $100 \mu \mathrm{m}$.

Differences in morphology between the cells cultured in each type of medium and in each type of hydrogel can be clearly seen in the 
different pictures shown, but it is more difficult to arrive at a conclusion about the higher or lower level of GAGs synthesis in the different conditions. We therefore carried out a quantitative analysis to study the percentage of cells expressing GAGs (Figure 4-29a) and a qualitative analysis to study the dark blue tone $(B L)$ and the blue area (BA) around the cell (Figure 4-29b). A high percentage of cells (around $90 \%$ ) cultured in the five types of Gel/HA in CM synthesized GAGs. In a qualitative analysis, a smaller and darker area around the cell was obtained with increased hyaluronic acid content, indicating a higher concentration of GAGs around the cell. This may be a consequence of the stiffness of the matrix, which is higher with larger amounts of hyaluronic acid in the hydrogel composition, the lower degradation rate of the hyaluronic acid chains that need more time to degrade and leave space for the matrix synthesized by the cells, the permeability of the hydrogel, the cell-hydrogel interactions and the presence of less cell adhesion sequences. In the cell-hydrogel system cultured in GM, the percentage of cells synthesizing GAGs increases with the percentage of HA in the hydrogel. As in the CM, the qualitative analysis shows, a higher BL with higher HA content. The BA around the cell is quite small in all cases, although it increased with higher HA content. 

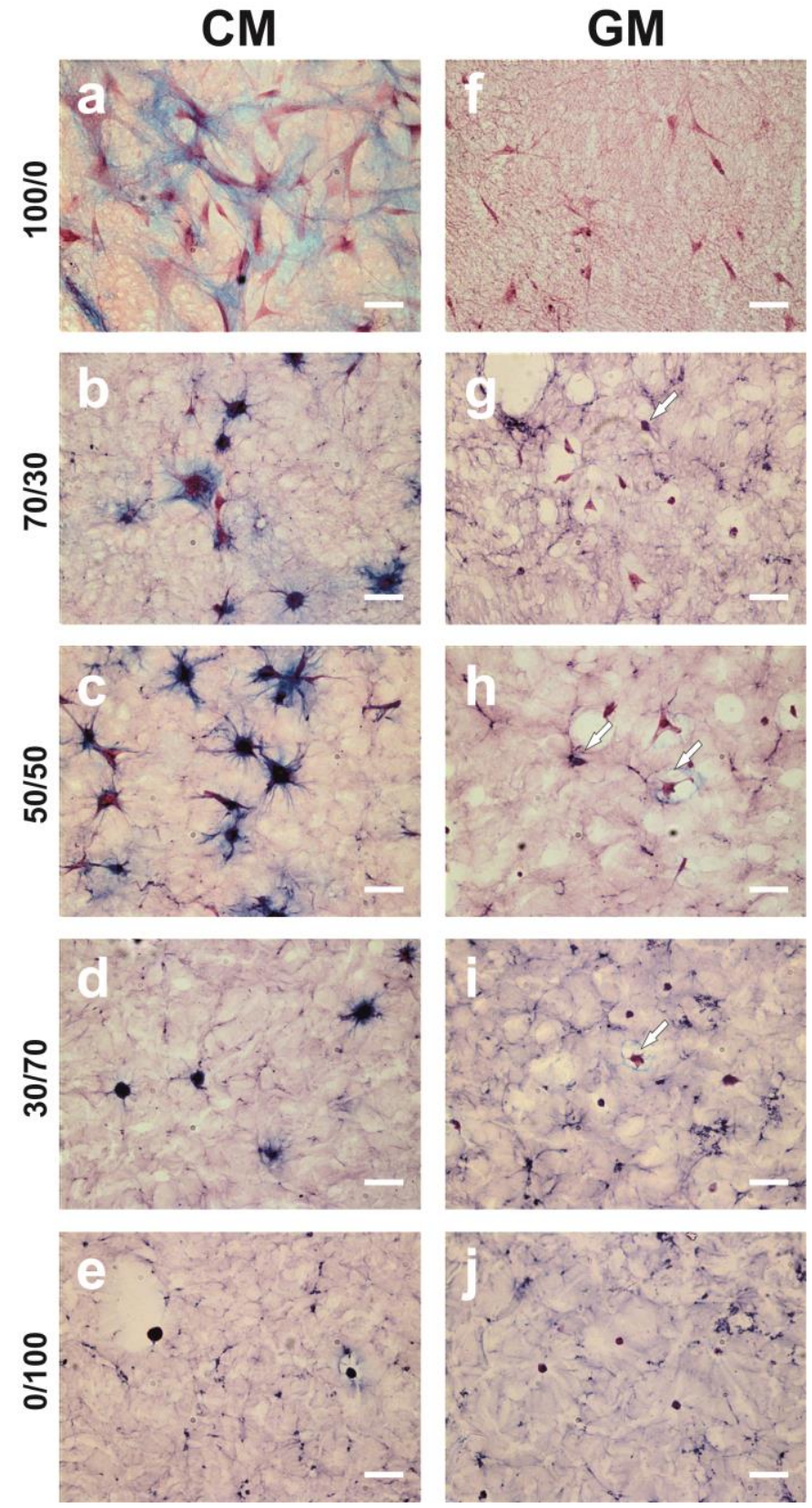

Figure 4-28. Alcian blue and nuclear fast red staining of Gel/HA hydrogels cultured in (a-e) chondrogenic medium and (f-j) in growth

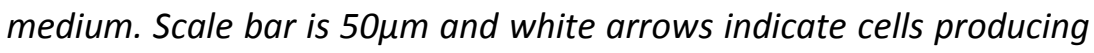
GAGs for the samples cultured in growth medium. 

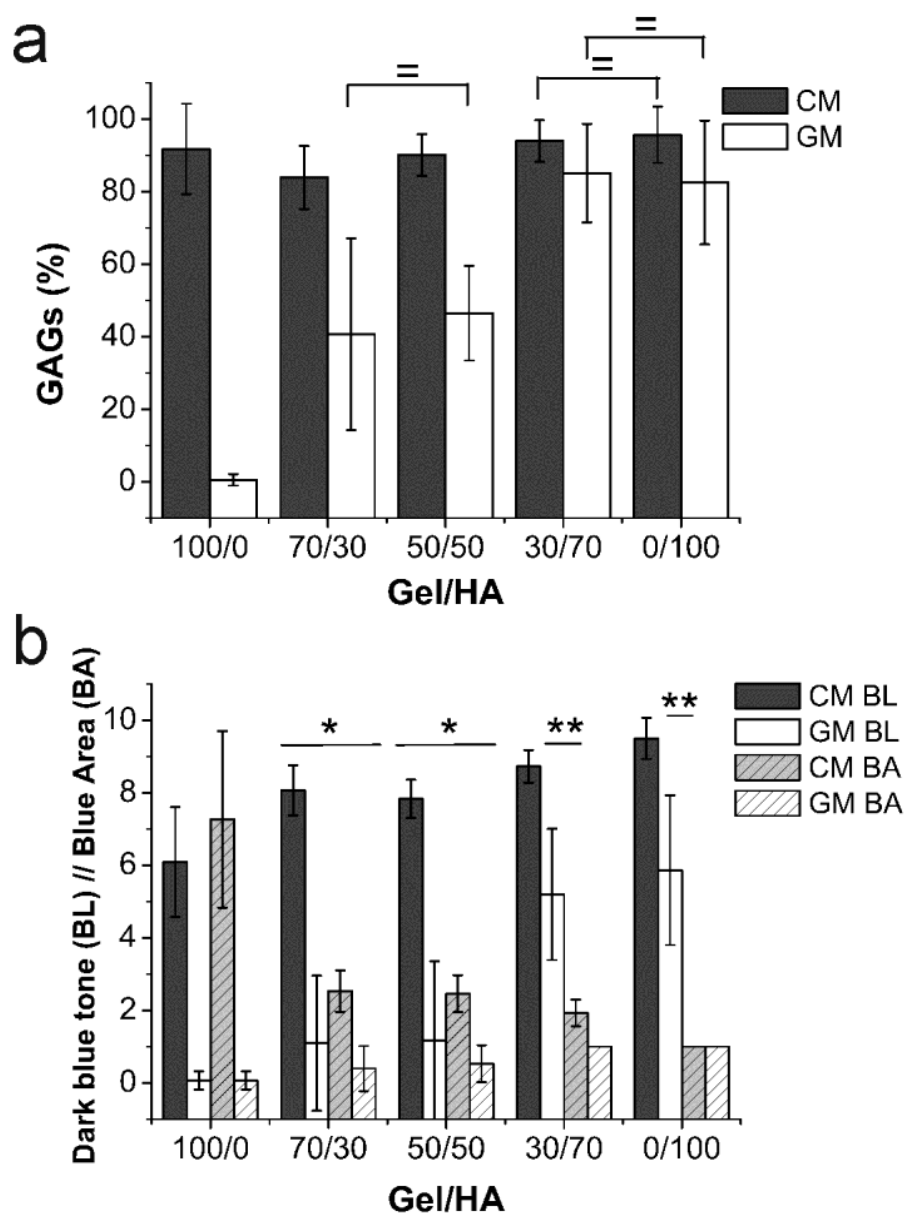

Figure 4-29. Alcian blue staining analysis of BM-hMSCs embedded in Gel/HA hydrogels and cultured in chondrogenic medium (CM) or in growth medium (GM) for: a) percentage of cells expressing GAGs, an equal sign marks the groups without significant differences; and b) dark blue tone or blue level (BL) and blue area (BA) around the cells for each Gel/HA group, * and ** mark the Gel/HA compositions without statistical significant differences within the same type of analysis. Maximum value of $B L$ and $B A$ is 10 . 


\subsubsection{Conclusions}

The immunohistochemical analysis of BM-hMSCs differentiation in GM showed positive staining for aggrecan in all Gel/HA compositions and negative staining for STRO-1, RUNX2, MF-20 and LPL. The percentage of cells expressing aggrecan increased with HA content. GAGs synthesis was obtained for all the samples, except for pure gelatin, and the percentage of cells expressing GAGs increased in the hydrogels with higher HA content. The RGD sequences present in gelatin influence the cell proliferation rate and thus the number of cells within the Gel/HA hydrogels. Pure gelatin has a high number of cells, while there are no differences in the number of cells when Gel and HA are combined. There was no cell proliferation in pure hyaluronic acid hydrogels.

Gel and HA hydrogel hybrids show promise as networks for the regeneration of articular cartilage, as chondrogenic differentiation is promoted even in a growth medium. Moreover, the presence of HA seems to enhance aggrecan and GAGs synthesis. Gelatin is needed in the hybrids to improve cell proliferation, as very few cells were found in the pure hyaluronic acid hydrogels. 


\section{Chapter 5 Synthetic IPNs}

as ex vivo platforms for cartilage regeneration 



\subsection{Summary*}

The implementation of bioreactor assays before passing from biological tests under static in vitro conditions to in vivo trials with animals would reduce many trial-and-error assays in animal experiments to test scaffolds that may never reach the market, with the associated reduction in costs. However, the present bioreactors do not take into account that the scaffold will be confined by the healthy host tissue in vivo, and that the role of this tissue is important to properly study the feasibility of a scaffold [237]. The host tissue has previously been taken into account in an animal model by implanting a scaffold in the back of mice inserted in a piece of osteochondral tissue [1]. The main subject of this chapter is the synthesis of a series of reinforced biostable hydrogels in order to select the optimum material composition of a host-tissue-platform in a bioreactor for cartilage regeneration.

IPN synthesis was used to obtain reinforced hydrogels made of a hydrophilic network constrained by a hydrophobic network. The combination of a hydrophobic and hydrophilic polymer can be considered a suitable system to control hydrogel properties [238][242]. Hydrophobic polymers have good mechanical properties, but they lack water absorption capacity. In contrast, hydrophilic polymers have better wettability and water permeability, but reduced loadbearing capacity. The interaction between the hydrophobic and hydrophilic components can therefore produce a compound with enhanced properties

Poly(2-hydroxyethyl acrylate) (PHEA) was selected as the hydrophilic network for its good biocompatibility, elastomeric behavior, water sorption capacity and the possibility of being reused in the bioreactor. The hydrophobic poly(ethyl acrylate) (PEA) network, was used to reinforce the hydrogel through the synthesis of sequential IPNs. The quantity of PHEA was regulated by changing the porosity of the first PEA network through polymerization with different quantities of ethanol. In addition, we used triethylenglycol 
dimethacrylate (TEGMA) as a more flexible crosslinker than the shorter frequently used ethylene glycol dimethacrylate molecule (EGMA) [238], [243], [244].

Differential Scanning Calorimetry (DSC) and Dynamic Mechanical Spectroscopy (DMS) assays were carried out to study the morphology of the hydrogels. The hydrogels' swelling capacity and diffusion coefficient were calculated. Unconfined compression assays and stress relaxation assays were also performed to obtain the Young's modulus and permeability of the materials, which made possible to select the IPN with values within the range of articular cartilage [11] for the bioreactor platform. Finally, the microstructure of the selected IPN was observed by cryoSEM.

*The results presented in this chapter have been published in:

Poveda-Reyes, S., Gamboa-Martínez, T. C., Manzano, S., Doweidar, M. H., Gómez Ribelles, J. L., Ochoa, I. and Gallego Ferrer, G. Engineering Interpenetrating Polymer Networks of Poly(2Hydroxyethyl Acrylate) as Ex Vivo Platforms for Articular Cartilage Regeneration. Int J Polym Mater, 64:14, 745-754 (2015). 


\subsection{Materials and methods}

\subsubsection{Materials}

Ethyl acrylate (99\%), triethylene glycol dimethacrylate (95\%) and 2hydroxyethyl acrylate (96\%) were provided by Sigma-Aldrich, Germany. Benzoine, ethanol and potassium iodide (extrapure) were purchased from Scharlab, Barcelona, Spain.

\subsubsection{Synthesis of the hydrogel interpenetrating polymer networks (IPNs)}

To obtain the first hydrophobic network, ethyl acrylate (EA) was polymerized in the presence of ethanol and with $5 \% \mathrm{w} / \mathrm{w}$ triethylene glycol dimethacrylate (TEGMA) as crosslinker and $0.5 \% \mathrm{w} / \mathrm{w}$ of benzoine as initiator, relative to the mass of EA and ethanol (see the chemical structures and the initiation of the polymerization in Figure 5-1). The porosity in the network was modulated by adding different quantities of ethanol to the solution $(0,20,40,50$ and $60 \% \mathrm{w} / \mathrm{w})$. The solutions were poured between two glass plates separated with a Teflon ring of $2 \mathrm{~mm}$ thickness, sealed with clamps, and polymerized in a UV oven for $24 \mathrm{~h}$. Then, the PEA polymer was unmolded and washed in boiling ethanol for three days, changing the ethanol daily. Finally, the samples were dried for two days at room temperature and then under vacuum at $80^{\circ} \mathrm{C}$ until constant weight.

To obtain the interpenetrating networks, different pieces of dried PEA films of known dry weight $\left(m_{P E A}\right)$ were swollen for 2 days in a solution of $60 \% \mathrm{w} / \mathrm{w}$ 2-hydroxyethyl acrylate (HEA) and $40 \% \mathrm{w} / \mathrm{w}$ ethanol (HEA60/40). The solution also contained a $5 \% \mathrm{w} / \mathrm{w}$ of TEGMA and $0.5 \% \mathrm{w} / \mathrm{w}$ of benzoine. The HEA the monomer chemical structure and initiation of the polymerization can be seen in Figure 5-1. The swollen pieces of films were placed between the above mentioned glass plates, separated by the Teflon ring sealed with clamps, and introduced again in the UV oven for $24 \mathrm{~h}$ to polymerize the PHEA second network. Then, the IPNs were unmolded and washed in 
boiling ethanol for three days changing the solvent every day. Finally, they were dried for two days at room temperature and then under vacuum at $80^{\circ} \mathrm{C}$ until constant weight. This final weight $\left(m_{I P N}\right)$ allowed calculating the PHEA mass percentage in the IPNs (\%PHEA) shown in Table 5-1 as follows, see Equation 15:

$\%$ PHEA $=\frac{m_{P H E A}}{m_{I P N}} \cdot 100=\frac{m_{I P N}-m_{P E A}}{m_{I P N}} \cdot 100$

a)
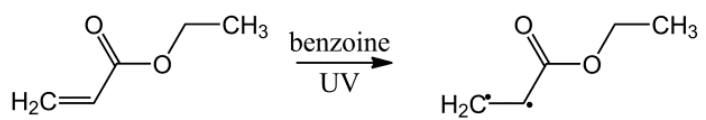

b)
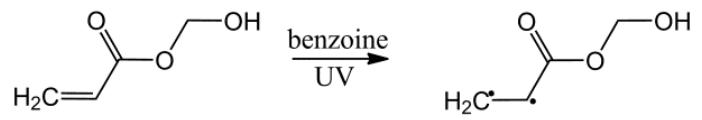

c)

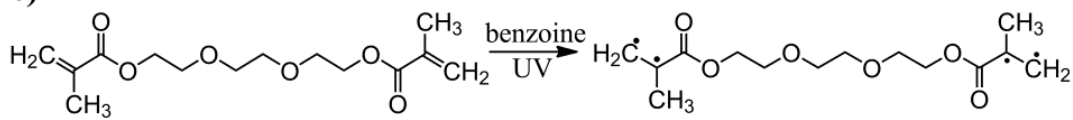

Figure 5-1. Chemical structure of: a) The hydrophobic ethyl acrylate monomer of the first network; b) the hydrophilic hydroxyethyl acrylate monomer of the second network and c) the triethylene glycol dimethacrylate monomer used in the crosslinking of each network. See also the initiation of the polymerization under ultraviolet light by benzoine.

The nomenclature used to identify each IPN is xEA-yEtOH, where $x$ is the percentage of EA and $y$ is the percentage of ethanol used to obtain the pores in the PEA network (Table 5-1); both are weight percentages. Hereafter, the different IPNs will be named with their PHEA mass fraction expressed in percentage.

Bulk and porous pure PHEA samples were also prepared following the same steps as in the synthesis of the PEA network. 5\% of TEGMA 
relative to the mass of monomer (monomer and ethanol for the porous one) was used in the synthesis. For the porous sample the HEA monomer was polymerized with $40 \% \mathrm{w} / \mathrm{w}$ of ethanol (PHEA60/40).

Table 5-1. Composition of the IPNs indicated by the mass percentage of PHEA in them (\%PHEA), glass transition temperature $\left(T_{g}\right)$ determined by the inflexion point of the first derivative of the DSC thermograms of the PEA and PHEA rich domains in the IPNs, and rubbery modulus at $100^{\circ} \mathrm{C}\left(\mathrm{E}^{\prime}\left[100^{\circ} \mathrm{C}\right]\right)$ of the IPNs.

\begin{tabular}{lcccc}
\hline IPNs & \% PHEA & $\begin{array}{c}\text { Tg }\left({ }^{\circ} \mathbf{C}\right) \\
\text { PEA- } \\
\text { rich } \\
\text { domains }\end{array}$ & $\begin{array}{c}\text { Tg }\left({ }^{\circ} \mathbf{C}\right) \\
\text { PHEA- } \\
\text { rich } \\
\text { domains }\end{array}$ & $\begin{array}{c}\mathbf{E}^{\prime}\left(\mathbf{1 0 0}^{\circ} \mathbf{C}\right) \\
(\mathbf{M P a})\end{array}$ \\
\hline $\begin{array}{l}\text { bulk PHEA } \\
\text { porous-PHEA } \\
\text { (PHEA60/40) }\end{array}$ & 100 & - & & 2.17 \\
40PEA-60EtOH & $79 \pm 2.00$ & -5.8 & 10.5 & 0.55 \\
50PEA-50EtOH & $71 \pm 2.12$ & -6.3 & 11 & 0.61 \\
60PEA-40EtOH & $64 \pm 3.05$ & -6.5 & 12.8 & 0.66 \\
80PEA-20EtOH & $53 \pm 1.35$ & -7.8 & 11 & 0.88 \\
100PEA-0EtOH & $46 \pm 5.31$ & -8.8 & 12.5 & 1.94 \\
bulk-PEA & 0 & -8.3 & - & 1.88 \\
\hline
\end{tabular}

\subsubsection{Differential Scanning Calorimetry (DSC)}

Differential Scanning Calorimetry measurements were carried out on dry samples in a DSC 823e calorimeter (Mettler-Toledo International Inc., Ohio, USA). The samples were sealed in aluminium pans just before the measurements began. All samples were subjected to a 
first heating scan from $25^{\circ} \mathrm{C}$ to $90^{\circ} \mathrm{C}$ at $5^{\circ} \mathrm{C} / \mathrm{min}$ to erase their thermal history. Then, they were cooled to $-100^{\circ} \mathrm{C}$ at a rate of $40^{\circ} \mathrm{C} / \mathrm{min}$ followed by an isotherm for $5 \mathrm{~min}$. A second heating scan was performed at $5^{\circ} \mathrm{C} / \mathrm{min}$ until $90^{\circ} \mathrm{C}$, which was considered for determination of the thermal properties of the systems.

\subsubsection{Dynamic Mechanical Spectroscopy (DMS)}

Dynamic Mechanical Spectroscopy (DMS) was performed on the dry samples in the tension mode at $2^{\circ} \mathrm{C} / \mathrm{min}$ in a Seiko DMS210 instrument (Seiko Instruments Inc., Chiba, Japan), from -130 to $120^{\circ} \mathrm{C}$ at a frequency of $1 \mathrm{~Hz}$. Specimens were prismatic shaped, approximately $25 \times 2 \times 0.7 \mathrm{~mm}^{3}$. The width of the sample for the DMS assay corresponds to the thickness of the obtained IPNs.

\subsubsection{Determination of water diffusion and equilibrium water sorption}

Water diffusion inside the networks was gravimetrically determined at room temperature by immersing the dry samples in water and measuring their weight gain at selected times until equilibrium was reached. The excess water on the surface of the specimens was carefully wiped away with absorbing paper before weighing. The weight of the samples was recorded in the following time intervals: up to 1.5 hours every 5 minutes, then up to 4 hours every 10 minutes and then at 4.5, 5, 6, 7 and 24 hours. Although equilibrium was reached at 24 hours, the weight of the samples was measured every day up to 4 days to confirm. The diffusion coefficient of water was determined assuming the Fick's law, according to Equation 16:

$$
\frac{\Delta m_{t}}{\Delta m_{\infty}}=\frac{4}{\sqrt{\pi}} \cdot \sqrt{\frac{t \cdot D}{e^{2}}},
$$

where $\Delta m_{t}$ and $\Delta m_{\infty}$ are the weight gains at time $t$ and at equilibrium, respectively, $e$ is the thickness of the sample, and $D$ the 
apparent diffusion coefficient. The slope of the $\frac{\Delta m_{t}}{\Delta m_{\infty}}$ vs. $\frac{\sqrt{t}}{e}$ allowed the values of $D$ [241], [243], [245] to be obtained. Moreover, values of equilibrium water content in immersion based on the weight of water per dry IPN mass $(w)$, and the mass of water per PHEA mass present in the IPN $\left(w^{\prime}\right)$, using equations 17 and 18, respectively, were calculated.

$$
\begin{gathered}
w=\frac{m_{\mathrm{H}_{2} \mathrm{O}}}{m_{\text {dryIPN }}} \\
w^{\prime}=\frac{m_{\mathrm{H}_{2} \mathrm{O}}}{m_{P H E A}}
\end{gathered}
$$

The equilibrium water sorption at $66 \%$ and $100 \%$ relative humidity $(\mathrm{RH})$ at $37^{\circ} \mathrm{C}$ was also measured. $66 \%$ relative humidity (RH66) was obtained with an aqueous supersaturated potassium iodide solution [246] in a closed tube, where the samples were placed without any contact with the solution. The $100 \% \mathrm{RH}$ (RH100) was reached in similar tubes by using pure water. Triplicate measurements were performed.

\subsubsection{Morphological structure by cryogenic scanning electron microscopy (cryo-SEM)}

To confirm some conclusions about the morphology of the systems, the 79\% PHEA IPN was immersed in water until equilibrium and properly treated to be observed in the cryogenic scanning electron microscope (cryo-SEM) JSM-6300. The sample was rapidly frozen in liquid nitrogen, and fractured in the cryo-transfer system vacuum chamber. After water sublimation at $-90^{\circ} \mathrm{C}$, the sample was coated with gold in the same chamber, directly moved into the SEM column and examined. 


\subsubsection{Monotonic uniaxial compression tests}

Uniaxial unconfined compression test in immersion in phosphate buffered saline solution (PBS) was performed in an InstronMicroTester 5548 machine (Instron, Massachusetts, USA) with a precision of $0.0001 \mathrm{~N}$ and $0.001 \mathrm{~mm}$ in force and displacement, respectively. A monotonic ramp at $1 \mathrm{~mm} / \mathrm{min}$ cross-head velocity was carried out with a $50 \mathrm{~N}$ load cell. Cylinders measuring $6 \mathrm{~mm}$ in diameter and $2 \mathrm{~mm}$ high were punched from larger pieces of the material. After drying in vacuum for 24 hours at $80^{\circ} \mathrm{C}$ the samples were hydrated up to equilibrium in a phosphate buffered saline solution (PBS) at $37^{\circ} \mathrm{C}$. The sample dimensions were monitored before and after the test. Young's modulus $\left(E_{S}\right)$ was calculated from the slope of the linear region in the stress-strain curve using the initial cross-section area [247], [248]. Each test began with a predeformation of $4 \%$ at a displacement rate of $0.001 \mathrm{~mm} / \mathrm{s}$ [249], [250] to eliminate nonlinearity generated by the geometrical variability of the sample and to normalize an initial reference test point. In order to start at the same preconditioning point, a first contact of the test tool with the sample was performed, taking a force ranging between $0.01 \mathrm{~N}$ and $0.02 \mathrm{~N}$ [251], [252] as a reference. At least three replicates per sample type were measured.

\subsubsection{Charge-relaxation compression tests to determine the permeability of the IPNs}

Similar preconditioning conditions and sample dimensions as in the uniaxial compression test were selected [253], [254], but in this case confined compression was performed. The confinement chamber has an exclusively permeable surface at the bottom, and the fluid is forced to flow towards this area [255]. Samples were subjected to constant deformation steps at $0.001 \mathrm{~mm} / \mathrm{s}$ up to determined strains $(5 \%, 8 \%, 11 \%, 14 \%, 17 \%, 20 \%$ and $23 \%)$ each of them followed by relaxation periods where the sample was maintained in the achieved deformation position for 15 minutes in the Instron MicroTester 5548 machine. The stress-strain curve allows obtaining the aggregated 
modulus $\left(H_{a}\right)$ of the material by a linear fit to the points obtained at the end of each relaxation period [249]. The relaxation curves were then obtained by representing the force $(F)$ versus time $(t)$ of each relaxation period. Exponential fitting to the experimental points by minimal squares was used to obtain the relaxation time $(\tau)$ as follows in Equation 19:

$$
F(t)=F_{\text {int }}+F_{0} \cdot e^{-\frac{t}{\tau}}
$$

where $F(t)$ is the applied force time dependent and $t$ is the time of the fitted range. $F_{\text {int }}$ is the force at maximal relaxation corresponding to $t$ equal to infinite, considered zero for simplicity. $F_{0}$ is the force at maximal stress $(t=0)$ and $\tau \neq 0$. Material permeability $(k)$ was obtained as the average of the permeability values calculated at each relaxation period by using Equation 20:

$$
k=\frac{h}{H_{a} \cdot \tau \cdot \pi^{2}}
$$

$h$ being the thickness of the sample [256].

Samples were punched to a diameter of $6 \mathrm{~mm}$ after reaching equilibrium in PBS. Experiments were performed in triplicate and with the specimens immersed in PBS to simulate physiological conditions.

\subsubsection{Statistical analysis}

Data were analyzed using Statistica 5.0 (Statsoft software), and statistical significance was set at $p<0.05$. Results are presented as mean \pm standard error. The normal distribution was tested by the Kolmogorov-Smirnov test. For parametric comparison, one way ANOVA with the Bonferroni post hoc tests was performed. For nonparametric comparisons, a Kruskal-Wallis test was performed followed by the Mann-Whitney U-test. 


\subsection{Results}

\subsubsection{Differential scanning calorimetry (DSC)}

Figure 5-2a shows the heating DSC thermograms for the different dry IPNs and the pure networks (bulk PEA and porous PHEA). One step on the normalized heat flow corresponding to their glass transition is observed for the pure samples. The inflexion point of this curve was considered as the value of $T_{g}$ (seen as a minimum in the first derivative curve of the normalized heat flow, Figure 5-2b), being equal to $-8.3^{\circ} \mathrm{C}$ for the pure bulk PEA network and $12.3^{\circ} \mathrm{C}$ for the pure porous PHEA network. Two glass transitions are clearly seen in the DSC thermograms of the IPNs with lower PHEA content (46\%, 53\% and $63 \%$ of PHEA) that are attributed to the PEA rich and PHEA rich domains in the IPNs, respectively. In the case of the IPNs with higher amounts of PHEA (71 and $79 \%$ of PHEA), the two $T_{g}$ steps are almost undetectable due to their proximity in temperature, the decrease in intensity of the transition of the PEA domains and the overlap with the transition of the PHEA rich domains. The two glass transitions are better detected in the first derivative curve in Figure $5-2 b$ which are tabulated in Table 5-1. A slight displacement of the glass transition temperature of the PEA rich domains in the IPNs to higher values is observed as the percentage of ethanol used in the polymerization of the first network is increased. This movement is due to the slight increase of the crosslinking density of the PEA network, as the TEGMA percentage was calculated proportionately to the EA and ethanol solution. In the case of the PHEA rich domains the glass transition temperature is constant and similar to the $T_{g}$ of the pure porous PHEA network. 
a)

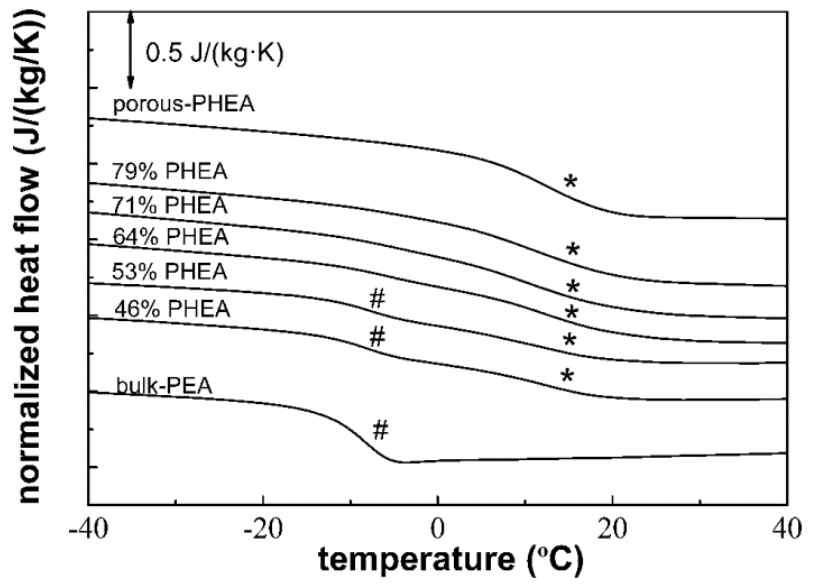

b)

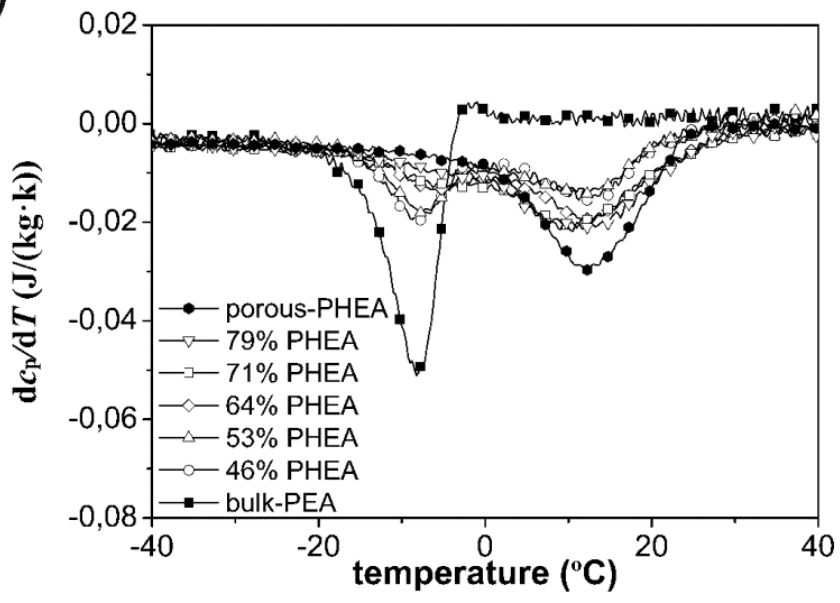

Figure 5-2. a) Heating DSC thermograms of dry samples at a rate of 5 $\mathrm{K} / \mathrm{min}$. The symbols \# and * indicate the $\mathrm{Tg}$ of the PEA and PHEA rich domains, respectively. b) $d c p / d T$ as a function of the temperature of dry IPNs, pure bulk PEA and porous PHEA.

\subsubsection{Dynamic Mechanical Spectroscopy (DMS)}

Figure 5-3a shows the temperature dependence of the Young's storage modulus $\left(E^{\prime}\right)$ of the samples and Figure 5-3b the corresponding curves of the loss tangent. The Young's storage 
modulus of the samples in the glassy state has values from $2.2 \times 10^{3}$ to $4.8 \times 10^{3} \mathrm{MPa}$ without any trend observed with the PHEA mass fraction in the IPNs (Figure 5-3a). Above $-25^{\circ} \mathrm{C}$ the Young's storage modulus sharply decreases due to the main relaxation. PEA polymeric chains are the first ones to change their state (at about $0^{\circ} \mathrm{C}$ ) and the last are the PHEA chains (at around $37^{\circ} \mathrm{C}$ ); with intermediate behaviour being observed in the IPNs. At temperatures higher than $75^{\circ} \mathrm{C}$ the characteristic plateau of rubbery networks is observed. The rubbery moduli of bulk PEA, bulk PHEA and the IPN with the lower PHEA content (46\%) are almost the same, $1.88 \mathrm{MPa}$ for bulk PEA, 2.17 MPa for bulk PHEA and 1.94 MPa for the 46\% PHEA IPN (Table 51). However, the porous PHEA network has the lowest value of the rubbery modulus because polymerization with ethanol produces a porous hydrogel, $0.17 \mathrm{MPa}$. A similar fall is observed for the IPNs that were obtained using PEA networks with some porosity (IPNs with PHEA contents $53 \%, 64 \%, 71 \%$ and $79 \%$ ) in which the rubbery modulus has intermediate values between the non-porous PEA network and the porous PHEA network (PHEA60/40), the values decreasing as the PHEA content increases (Table 5-1).

For bulk PEA and porous PHEA the main $\alpha$ relaxation associated with glass transition is detected (Figure $5-3 b$ ), with peaks occurring at $2^{\circ} \mathrm{C}$ and $37^{\circ} \mathrm{C}$, respectively. For the IPNs with 46 and $53 \%$ of PHEA a small shoulder due to the $\alpha$ relaxation of the PEA rich domains can be detected, which is hidden in the IPNs with higher amounts of PHEA by its overlapping with the main relaxation of the PHEA rich domains. This peak appears at lower temperatures than the main relaxation peak of the pure porous PHEA network. However, it shifts to higher temperatures with the increase of the PHEA content in the IPNs, and for IPN 79\% the maximum coincides with that of the PHEA pure sample. A slight broadening of this peak is also observed for the highest PHEA contents which could be due to an increase in the IPN porosity, as previously observed in PHEA networks of different porosities [257], [258]. 
a)

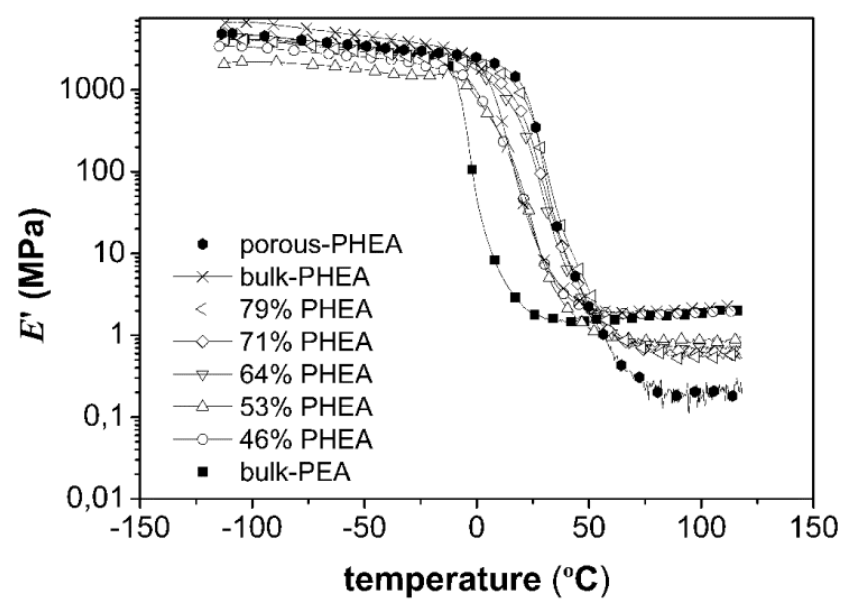

b)

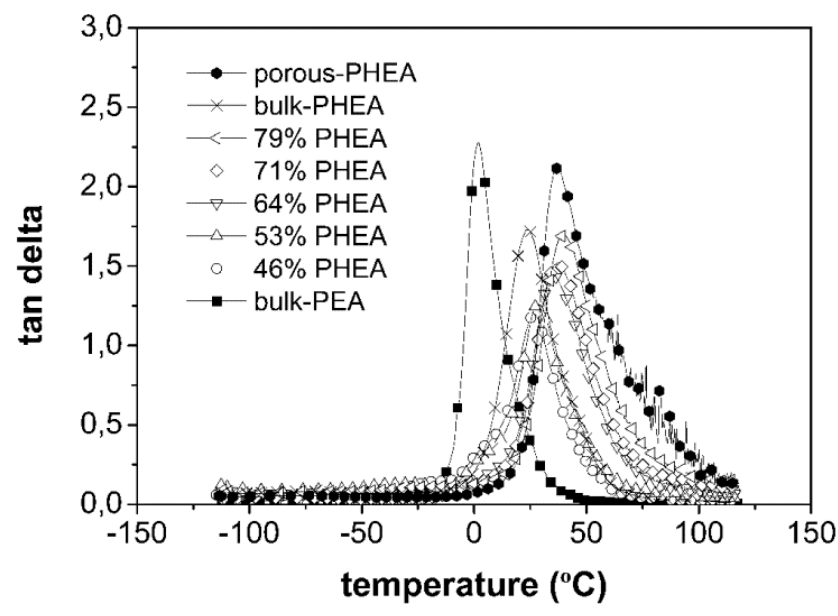

Figure 5-3. Dynamic-mechanical Young's storage modulus ( $\left.E^{\prime}\right)(a)$ and loss tangent (tan delta) (b) as a function of temperature at $1 \mathrm{~Hz}$ for the dry IPNs and pure networks.

\subsubsection{Equilibrium water sorption and diffusion of water}

Figure 5-4a shows the equilibrium water mass fraction of the different samples $(w)$ referred to the dry mass of the IPN at $66 \%$ and $100 \%$ relative humidity (RH 66 and $\mathrm{RH} \mathrm{100,} \mathrm{respectively)} \mathrm{and} \mathrm{in}$ immersion in water as a function of the PHEA percentage in the IPNs. When the samples are equilibrated in a water vapour environment 
and the activity of water is less than one (RH 66), the water content is the same for all the samples and no influence of the presence of PEA is observed. However, an increase in the value of $w$ with the PHEA content in the IPNs is obtained for water activity equal to unity (RH 100 and immersion). As expected, the porous PHEA sample absorbs a higher quantity of water than the bulk PHEA sample in immersion due to its porosity. The variation of the amount of water absorbed in the different IPNs can be explained because they contain different proportions of PEA (which is hydrophobic and barely absorbs water). To eliminate this effect we have expressed the amount of water absorbed in the IPNs by the mass of PHEA in them ( $w^{\prime}$ ) (Figure 5-4b). In this new representation and when the samples are equilibrated in a vapour environment the water absorption capacity of the PHEA in the IPNs is the same as that of the pure bulk PHEA network, regardless of water activity ( $\mathrm{RH} 66$ and $\mathrm{RH}$ 100). This similar behaviour is typical of phase-separated systems [244].

The amount of water that PHEA absorbs in gaseous environments does not lead to a significant expansion of the network and the constraining effect of the PEA network is not observed. By contrast, the swelling in liquid water causes a greater expansion of the network and the opening of its pores, with the formation of pure water domains both in the hydrogel phase and in its pores [259], [260]. It is in this environment where differences in behavior between the PHEA in the IPNs and pure PHEA are noticed, the constraint of PHEA expansion caused by its interpenetration with the PEA network being most remarkable. Thus, $w^{\prime}$ values for the IPNs with $46 \%, 53 \%$ and $64 \%$ of PHEA are lower than for pure bulk PHEA (see Figure 5-4b). Noticeably, the value of $w^{\prime}$ obtained for the IPN $79 \%$ is higher than the value in pure bulk PHEA, although lower than that of the porous PHEA sample (Figure 5-4b). Such a high water absorption capacity is only possible if the PHEA phase in the IPN is porous. Indeed, the cryoSEM photograph presented in Figure 5-4 confirms that this IPN is porous, with pore size of approximately 160 $\mathrm{nm}$. This porous structure has been seen in other PEA-i-PHEA IPNs 
and happened only when polymerization of the second network occurred in the presence of ethanol [243]. Porosity measures are outside the scope of this work, however the value of volume fraction of pores should be somewhat below than the value found in [257] for PHEA synthesized with $40 \%$ ethanol and using EGMA as crosslinker (i.e lower than 0.32).
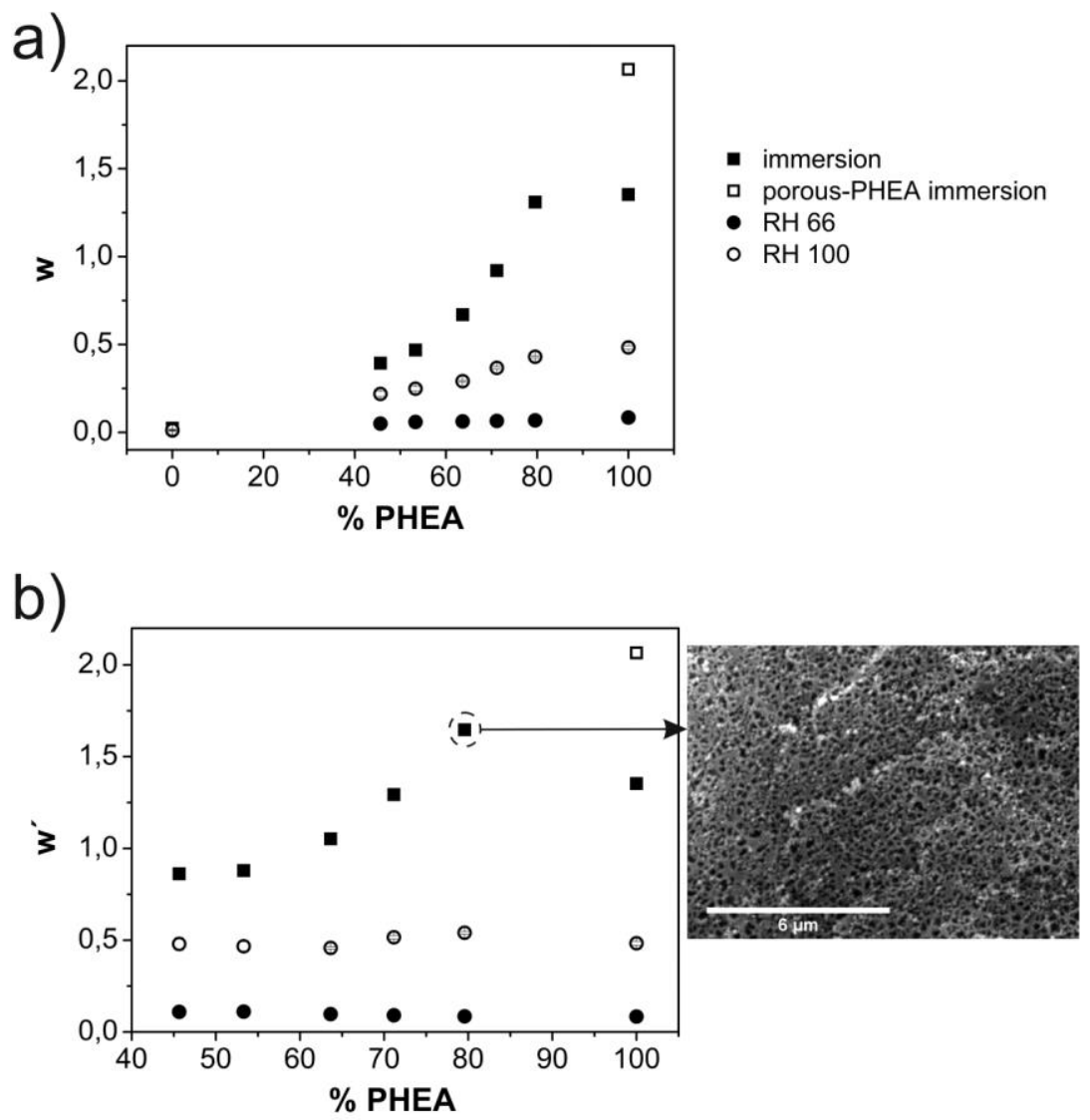

Figure 5-4. a) Water content per mass of dry IPN (w) at 66\% (RH 66) and $100 \%(\mathrm{RH} 100)$ relative humidity and swollen in water (immersion). b) Water mass per mass of PHEA in the IPN ( $W^{\prime}$ ) at $66 \%$ $(\mathrm{RH} 66)$ and $100 \%(\mathrm{RH} 100)$ relative humidity and swollen in water (immersion). The value for 100\% PHEA at RH 66\% and $\mathrm{RH} 100 \%$ are for bulk PHEA and in immersion in water are for bulk PHEA (full square) and porous PHEA (empty square). The picture corresponds to the cryo-SEM image of the IPN with 79\% PHEA. 
Figure 5-5 shows the apparent diffusion coefficient $(D)$ versus PHEA content for the IPNs immersed in water and for pure bulk PHEA at a relative humidity of $100 \%$ (RH 100) assuming Fickian behavior. Bulk PEA and bulk PHEA have a similar diffusion coefficient, which is the highest of all samples. Porous PHEA has a lower diffusion coefficient than bulk PHEA. When PHEA is interpenetrated with PEA $D$ is lower than the diffusion coefficient of the pure network and it decreases surprisingly with the increase of the PHEA hydrophilic component. This decrease is so pronounced that for the IPN with 79\% PHEA $D$ value is more typical of a diffusion of water in vapor environment than in a liquid environment [243], [244]. To confirm this, we measured and represented in the same graph for comparison, the value of water diffusion coefficient of bulk PHEA in a saturated vapor atmosphere ( $\mathrm{RH} 100$ ) (see the open symbol in Figure 5-5).

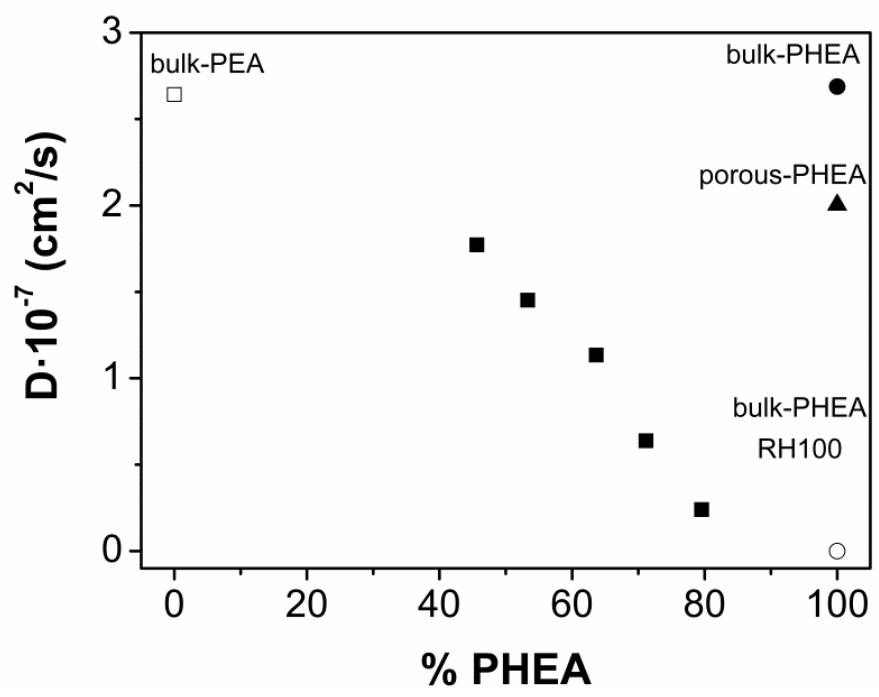

Figure 5-5. Diffusion coefficient of water in the IPNs, bulk PEA, bulk PHEA and porous PHEA in an immersion experiment. The empty circle corresponds to the diffusion coefficient of water in bulk PHEA in saturated vapor environment ( $R H$ 100). 


\subsubsection{Young's modulus in compression $\left(E_{S}\right)$ and permeability (k)}

The Young's moduli $\left(E_{S}\right)$ obtained for the different IPNs are shown in Figure 5-6a. As expected, since measurements are performed with the samples immersed in water, the increase in the percentage of PHEA in the IPNs results in the decrease of the $E_{S}$. The maximum value for $E_{S}, 1.45 \pm 0.13 \mathrm{MPa}$, was reached for the pure PEA network. Significant variations for $E_{S}$ were found when increasing the amount of PHEA.

Figure 5-6b shows how the increment of PHEA content in the IPNs generates an increment in the material's permeability. The results show that the pure porous PHEA sample, displayed a maximal permeability of $13.41 \times 10^{-16} \pm 0.29 \times 10^{-16} \mathrm{~m}^{4} /(\mathrm{N} \cdot \mathrm{s})$. The rest of the samples with lower PHEA percentages offered lower values of permeability, ranging from $8.68 \times 10^{-16} \pm 2.87 \times 10^{-16} \mathrm{~m}^{4} /(\mathrm{N} \cdot \mathrm{s})$ to $2.95 \times 10^{-16} \pm 1.12 \times 10^{-16} \mathrm{~m}^{4} /(\mathrm{N} \cdot \mathrm{s})$. 

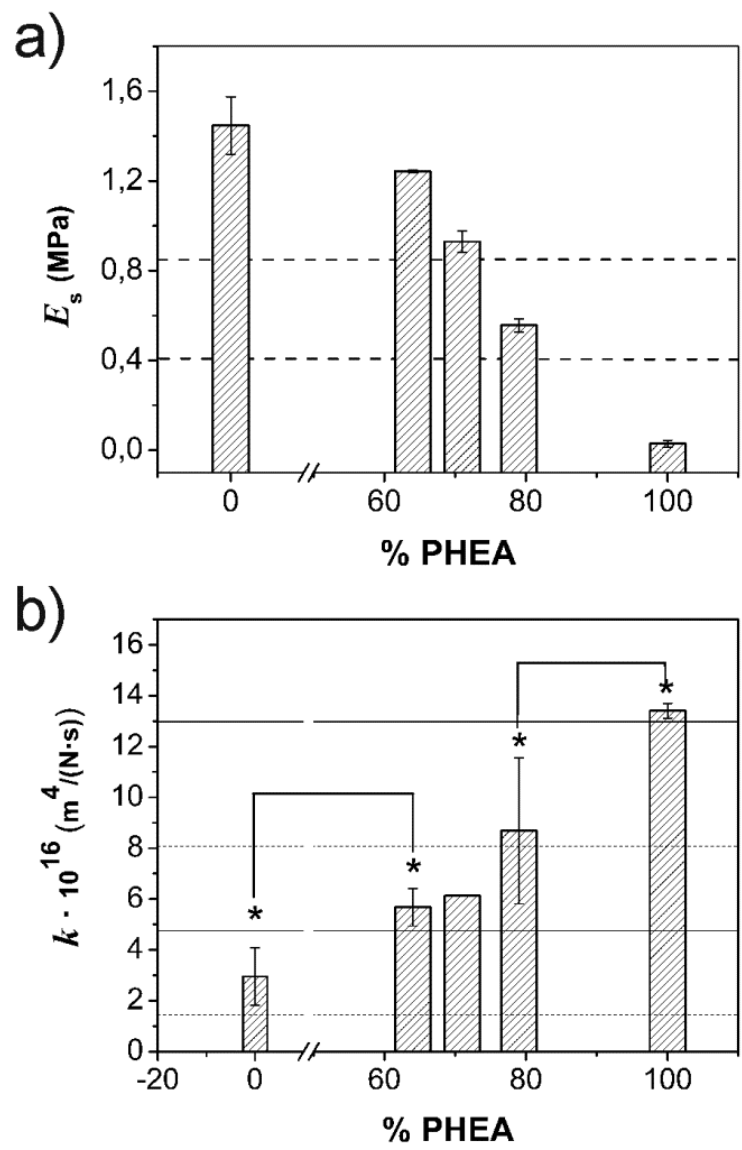

Figure 5-6. (a) Young's modulus $\left(E_{S}\right)$ and (b) permeability ( $k$ ) obtained for bulk PEA, porous PHEA and IPNs. Dash lines in a) indicate the normal limit values of $E_{S}$ found in literature for human knee articular cartilage [261]-[263]. Lines in b) indicate the range of experimental values of $k$ for human knee articular cartilage found in [264] from permeability tests (dash lines) and from creep tests (solid lines). 


\subsection{Discussion}

Polymerization of EA and TEGMA in the presence of a sufficiently large amount of diluent such as ethanol yields a porous polymer network. This is because microsyneresis takes place during polymerization, and at a certain conversion degree, ethanol is segregated in nano or micro drops inside the growing polymer network. When this first network is swollen in HEA, again with a certain amount of diluent, the monomer in part swells the first polymer network and in part fills its pores. After polymerization, the second network partially interpenetrates the PEA network and is partially in the form of dispersed pure PHEA domains occupying the volume of the pores initially formed in the first network (Figure 5-7). Interestingly, the PHEA network should be continuous, and covalently connect both regions, the interpenetrated one (IPN phase) and that of pure PHEA dispersed domains. This is expected to result in interesting mechanical performance of the material. On the other hand, as will be discussed below, pure PHEA domains occupying the pores of the first network can in turn be porous due to the presence of ethanol during polymerization. 


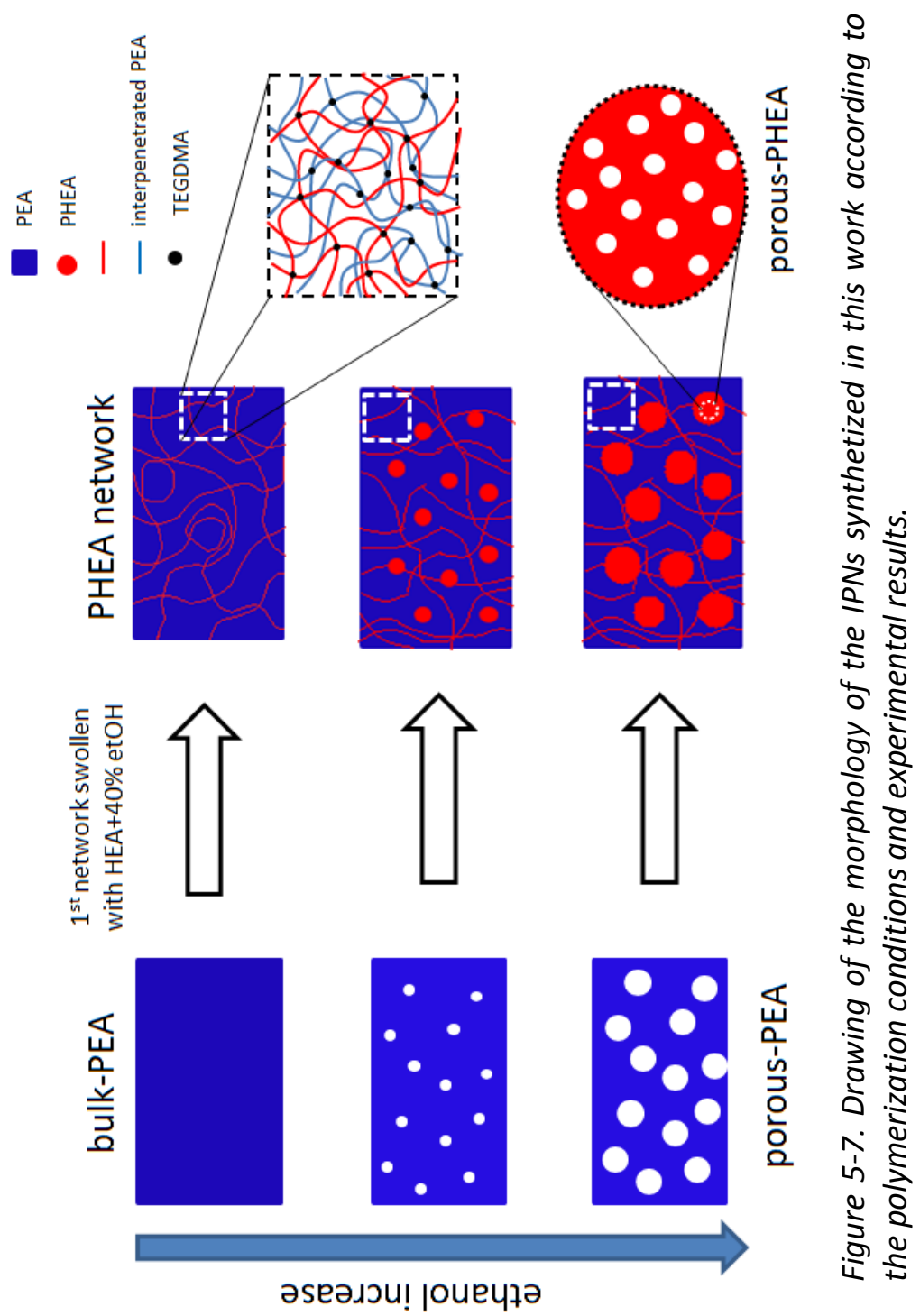

The conformational mobility of the polymer chains forming the IPNs, which is related to the nanostructure of the systems, has been studied by DSC and DMS. PEA and PHEA are immiscible polymers and their sequential IPNs form phase-separated systems for low crosslinking densities [238], [244]. However, forced compatibility can be obtained by using high quantities of crosslinker in the polymerization of the first network, resulting in systems where the 
polymeric chains of both polymers are perfectly interpenetrated, giving rise to one single relaxation in DMS at a temperature between those of the pure components [265], [266]. If the quantity of crosslinker in the first network is low, when the polymeric chains of the second network start growing during polymerization, they push the polymeric chains of first network apart forming phase-separated systems. Phase separated IPNs contain domains rich in their components (in our case PEA and PHEA) which relax at the same temperature as the corresponding pure systems and two relaxations are observed in the DMS spectra [266].

The DSC and DMS spectra, represented in Figure 5-2b and Figure 5$3 \mathrm{~b}$, respectively, show two relaxation peaks, one corresponding to the PEA rich domains and the other to the PHEA rich ones and confirm the phase separation phenomenon in the present IPNs. The displacement of the $T_{g}$ of the PEA rich domains to higher temperatures with the increase of PHEA content in the IPNs (Figure 5-2 and Table 5-1) is mainly the effect of the increase in the quantity of crosslinker that increased with the quantity of ethanol in the polymerization of the first network [266]-[268]. The $T_{g}$ of the PHEA rich domains determined by DSC seems to be independent of the quantity of PHEA in the IPNs (Figure 5-2 and Table 5-1). However for the IPNs with low PHEA content the main DMS relaxation temperature $\left(T_{\alpha}\right)$ of the PHEA rich domains takes place at temperatures below that of the pure porous PHEA network and shifts to higher temperatures with the increase of PHEA in the IPNs, until it almost coincides with that of pure porous PHEA for the $79 \%$ IPN. This phenomenon could be attributed to partial miscibility of the two components in the IPNs [268], [269], especially for those samples in which the PEA first network is not very porous and has a considerable quantity of TEGMA, $5 \%$ or higher, which is the case of the $46 \%, 53 \%$ and $64 \%$ IPNs. For those samples where the PEA first network is more porous, even if this first network has a slightly greater amount of crosslinker, the predominant PHEA phase is located in the pores (Figure 5-7). The relaxation peak of those domains partially mixed 
with the PEA network, if present, is overlapped by the main relaxation of the more abundant pure PHEA domains, which almost coincides with that of the pure PHEA sample.

The porosity of the systems causes a decrease of the rubbery modulus [257], [258], as seen in Figure 5-3 for the porous PHEA sample that has a considerably lower modulus than bulk PHEA (0.17 $\mathrm{MPa}$ for the porous sample versus $2.16 \mathrm{MPa}$ for the bulk sample). In this way, those IPNs having certain porosity, in either the first network, the second or both, have values of the rubbery modulus, which decrease with the porosity (that is; proportional to the quantity of ethanol used in the synthesis of the first network). This is the case of the IPNs 53\%, 64\%, 71\% and 79\% (Figure 5-3, Table 5-1) that have intermediate values between pure bulk PEA and porous PHEA, resembling more the modulus of porous PHEA when increasing the quantity of ethanol in the first network. The IPN $46 \%$ has a modulus that coincides with that of bulk PEA and bulk PHEA, as corresponds to a system where no ethanol was used in the preparation of the first network.

Details about porosity of the PHEA phase in the IPNs are inferred from the values of equilibrium water content in immersion of the samples, expressed per unit mass of PHEA in the IPNs (Figure 5-4b) which are supported by the cryo-SEM micrograph in Figure 5-4. Thus, the IPN with higher content of PHEA, 79\%, which was synthesised in the network of PEA with the highest porosity, is a reinforced interpenetrated network separated into two phases, in which the hydrophilic one is porous and is therefore able to absorb a greater amount of water than pure hydrophilic bulk hydrogel. These special qualities, reinforced but quite hydrophilic, make this sample a good candidate for the bioreactor platform, as discussed below.

Although PEA is a hydrophobic polymer and absorbs a small amount of water, the diffusion process in water is as rapid as in the PHEA hydrophilic network, as indicated by the similar values of their water diffusion coefficients (Figure 5-5). On the other hand, although the 
porous PHEA system absorbs a higher amount of water than the bulk PHEA network (Figure 5-4b) diffusion of water is slower, as indicated by the lower diffusion coefficient found in the porous PHEA network in comparison to the bulk one in Figure 5-5. Similar results were observed in [257], [258] for similar PHEA sponges. In the dry state, the porous systems have a large amount of discontinuities generated by the collapse of their pores. These multiple interface surfaces add tortuosity to water and hinder its diffusion. This delay is added to the process of pores opening that also slows down water diffusion, giving rise to lower diffusion coefficients. For the IPNs, the diffusion coefficient decreases with the increase of PHEA content (although it has the same order of magnitude, $10^{-7} \mathrm{~cm}^{2} / \mathrm{s}$ ) reaching values similar to the diffusion coefficient of pure PHEA at relative humidity $100 \%$ for the IPN with 79\% PHEA. This is mainly an effect of the constriction effect of the PEA network that hinders the opening of the collapsed pores of the PHEA rich phases and retards the diffusion of water molecules inside the IPNs, as previously observed in similar PEA-iPHEA IPNs [243]. The more porous the hydrophilic domains in the IPNs are, the slower the diffusion process is, which explains the decrease in $D$ with the PHEA content in Figure 5-5.

In order to select the most appropriate system to perform the role of host cartilage tissue in a bioreactor, we paid attention to the experimental values of the Young's modulus at compression and permeability, since these are the parameters that most contribute to simulating the properties of natural tissue. Physiological values of Young's modulus at compression $\left(E_{S}\right)$ for human knee cartilage collected in literature ranged from $0.41 \mathrm{MPa}$ to $0.85 \mathrm{MPa}$, depending on age, sex and other genetic factors of the patient [261]-[263]. According to these data, represented as dotted lines in Figure 6a, only IPN 79\% includes Young's modulus within this range. The permeability of cartilage also depends on several factors and varies through the depth of the tissue. In vitro permeability of the human lateral tibial plateau cartilage, measured by Boschetti et al. [264] in a permeability test, ranged from $1.3 \times 10^{-16}$ to $8.01 \times 10^{-16} \mathrm{~m}^{4} /(\mathrm{N} \cdot \mathrm{s})$ and in 
a creep test from $4.74 \times 10^{-16}$ to $12.98 \times 10^{-16} \mathrm{~m}^{4} /(\mathrm{N} \cdot \mathrm{s})$. Both ranges are represented as lines in Figure $6 \mathrm{~b}$ together with the values of the IPNs. The increment of PHEA content in the IPNs generates an increase of their permeability, which was already expected due to the increase of their hydrophilicity and porosity. All the samples evaluated here showed values within one or the two intervals provided by Boschetti et al. except the pure porous PHEA network, which has a permeability outside the intervals. In particular, the IPN $79 \%$ has permeability that is close to the upper limit of human cartilage extracted from a permeability test and it is within the range of the human cartilage permeability measured by the creep test. Being this IPN the only one that has a Young's modulus in the range of human articular cartilage, it was selected for the bioreactor platform to play the role of artificial host tissue, since it presents more similarities to human cartilage than the other compositions.

\subsection{Conclusions}

Variations of the quantity of ethanol used in the synthesis of the first PEA network in sequential IPNs allowed obtaining PHEA hydrogels with regulated hydrophilicities and porosities. The use of $5 \%$ wt. of TEGMA in the synthesis of the first PEA network is not enough to obtain complete miscibility between hydrophobic and hydrophilic components, resulting in phase-separated systems with domains rich in both components. In the case of PHEA domains, the displacement of the main relaxation temperature in the DMS spectra to lower values with the decrease of the PHEA content is attributed to partial miscibility with PEA. Water diffusion coefficient of PHEA is decreased by its interpenetration with PEA. Young's modulus in compression of the water immersed IPNs decreases with the amount of PHEA content as a consequence of the increase in both hydrophilicity and porosity. As expected, the hydrophilic polymer is softer than the hydrophobic one, and the coupling of both in the form of IPN results in obtaining intermediate moduli. An increase of PHEA in the IPN results in an increase in the permeability coefficient of water being 
outside the intervals of human articular cartilage for pure porous PHEA hydrogel. The only material with a water permeability coefficient and Young's modulus in compression similar to articular cartilage was that with a $79 \%$ of PHEA. This IPN is the best candidate to simulate the host articular cartilage. 



\section{Chapter 6 \\ Conclusions}



Three different hydrophilic systems were developed in the course of this thesis and the following conclusions were reached:

\section{Injectable gelatin-fiber hydrogels}

1. Loose PLLA microfibers were successfully obtained by: $i$ ) milling the PLLA electrospinning mesh (PLLA-ES) and ii) projecting the PLLA solution in a non-solvent under turbulent flow (PLLA-HT). They dispersed well in the gelatin solution and did not compromise its injectability.

2. Gelatin reinforcement was only provided by the electrospinningmilled microfibers. Increasing the PLLA-HT microfiber content in the gelatin composite did not raise the Young's modulus under compression, probably due to fibers' agglomeration prior to crosslinking or to a bad fiber-matrix interaction.

3. A hydrophilic surface grafting of PHEMA onto the PLLA loose microfibers was successfully carried out by photopolymerization.

4. The hydrophilic grafting improved the interphase between the fibers and the gelatin matrix and consequently increased mechanical properties of gelatin. Gelatin composites with 1\% PLLA-PHEMA microfibers increased the shear storage modulus of gelatin by almost $300 \%$.

5. GEL-PLLA injectable fiber composites were not cytotoxic. PHEMA grafting onto the fiber's surface did not cause cell cytotoxicity.

6. The new injectable gelatin-PLLA microfiber composites are promising materials for the tissue engineering of cartilage.

\section{ECM inspired injectable gelatin-hyaluronic acid hydrogels}

7. Tyramine grafting onto the gelatin and hyaluronic acid chains was successfully achieved and hydrogel injectable hybrids combining both components at $1 \%$ and $2 \% \mathrm{w} / \mathrm{v}$ were obtained. 
8. The high viscosity of the high molecular weight HA solution lengthened the gelation time with higher HA content within the Gel/HA hybrids and formed a phase separated system. The lower viscosity of low molecular weight HA solutions had matched gelation time of HA and Gel and the hybrids were more homogeneous and did not present phase separation.

9. The EWC increased with a reduced polymer concentrations and increased HA content. Young's modulus under compression and shear loss modulus increased with the higher polymer concentration and higher HA content.

10. Enzymatic degradation in vitro with a collagenase-hyaluronidase medium showed higher degradation rates for gelatin, which was retarded with increasing amounts of hyaluronic acid in the mixtures.

11. C2C12 cells adhered better to gelatin hydrogels due to RGD adhesion sequences. This improved adhesion helped the cells to exert traction forces that caused shrinkage of the gelatin hydrogel at a concentration of $8 \times 10^{6}$ cells $/ \mathrm{mL}$, while the presence of HA in the hydrogel composition avoided hydrogel shrinkage and permitted the formation of myotubes throughout the entire hydrogel. Cells in pure HA hydrogel presented a round morphology due to the lack of adhesion sequences.

12. BM-hMSCs were homogenously dispersed in the Gel/HA at a concentration of $1 \times 10^{6}$ cells $/ \mathrm{mL}$. Pure gelatin presented high amounts of $h M S C s$, while no differences in the number of cells where obtained when Gel and HA were combined. On the other hand, no cell proliferation occurred in pure hyaluronic acid hydrogels.

13. Differentiation of $B M-h M S C s$ in GM showed positive staining for aggrecan in all Gel/HA compositions and negative staining for STRO1, RUNX2, MF-20 and LPL. Percentage of cells expressing aggrecan and GAGs increased with HA content.

14. The ECM inspired hydrogels of Gel/HA are potential materials for the tissue engineering of cartilage. 
Synthetic IPNs as ex vivo platforms for articular cartilage regeneration

15. Sequential IPNs of PEA-PHEA with different hydrophilicities and mechanical properties were obtained by varying the quantity of ethanol used in the synthesis of the first PEA network. The systems present phase separation with domains rich in PEA and PHEA.

16. Water diffusion and compression Young's modulus were reduced with higher PHEA content in the IPN, due to higher hydrophilicity and porosity of the system.

17. IPNs with more PHEA had higher permeability coefficients, being outside the intervals of human articular cartilage for pure porous PHEA hydrogel.

18. IPN with 79\% PHEA was selected as the most suitable for the bioreactor platform, since it was the only one that fulfilled the requirements of permeability and Young's modulus with values inside the range of articular cartilage. 


\section{Bibliography}

[1] Schüller GC, Tichy B, Majdisova Z, Jagersberger T, van Griensven M, Marlovits $S$ and Redl H. An in vivo mouse model for human cartilage regeneration. J. Tissue Eng. Regen. Med. 2: 202-209 (2008).

[2] Poole AR, Kojima T, Yasuda T, Mwale F, Kobayashi M and Laverty S. Composition and Structure of Articular Cartilage. Clin. Orthop. Relat. Res. 391S: 26-33 (2001).

[3] Zhang X, Blalock D and Wang J. Classifications and Definitions of Normal Joints. Osteoarthritis - Progress in Basic Research and Treatment (2015). DOI: 10.5772/59977

[4] Cohen NP, Foster RJ and Mow VC. Composition and dynamics of articular cartilage: structure, function, and maintaining healthy state. J. Orthop. Sports Phys. Ther. 28(4): 203-215 (1998).

[5] Muir H. Proteoglycans of cartilage. J. Clin. Pathol. Suppl. (R. Coll. Pathol). 12: 67-81 (1978).

[6] Kiani C, Chen L, Wu YJ, Yee AJ and Yang BB. Structure and function of aggrecan. Cell Res. 12 (1): 19-32 (2002).

[7] Baeurle SA, Kiselev MG, Makarova ES and Nogovitsin EA. Effect of the counterion behavior on the frictionalcompressive properties of chondroitin sulfate solutions. Polymer. 50(7): 1805-1813 (2009).

[8] Lee GM and Loeser RF. Interactions of the chondrocyte with its pericellular matrix. Cells Mater. 8: 135-149 (1998).

[9] Sophia Fox AJ, Bedi A and Rodeo SA. The basic science of articular cartilage: structure, composition, and function. Sports Health. 1(6): 461-468 (2009). 
[10] Little CJ, Bawolin NK and Chen X. Mechanical Properties af Natural Cartilage and Tissue-Engineered Constructs. Tissue Eng. Part B. 17(4): 213-227 (2011).

[11] Mow VC and Guo XE. Mechano-electrochemical properties of articular cartilage: their inhomogeneities and anisotropies. Annu. Rev. Biomed. Eng. 4: 175-209 (2002).

[12] Falah M, Nierenberg G, Soudry M, Hayden M and Volpin G. Treatment of articular cartilage lesions of the knee. Int. Orthop. 34(5): 621-630 (2010).

[13] H. B. Sun HB. Mechanical loading, cartilage degradation, and arthritis. Ann. N. Y. Acad. Sci. 1211: 37-50 (2010).

[14] Umlauf D, Frank S, Pap T and Bertrand J. Cartilage biology, pathology, and repair. Cell. Mol. Life Sci. 67(24): 4197-4211 (2010).

[15] Zhang Y and Jordan JM. Epidemiology of Osteoarthritis. Clin. Geriatr. Med. 26(3): 355-369 (2010).

[16] Redman SN, Oldfield SF and Archer CW. Current strategies for articular cartilage repair. Eur. Cell. Mater. 9:. 23-32 (2005).

[17] Brodkin KR, García AJ and Levenston ME. Chondrocyte phenotypes on different extracellular matrix monolayers. Biomaterials. 25(28): 5929-5938 (2004).

[18] Bhardwaj N, Devi D and Mandal BB. Tissue-Engineered Cartilage: The Crossroads of Biomaterials, Cells and Stimulating Factors. Macromol. Biosci. 15(2): 153-182 (2015).

[19] Chung C and Burdick JA. Engineering cartilage tissue. Adv. Drug Deliv. Rev. 60(2): 243-62 (2008).

[20] Jacobi M, Villa V, Magnussen RA and Neyret P. MACl - a new era? Sport. Med. Arthrosc. Rehabil. Ther. Technol. 3(1):10 (2011). 
[21] Luo Y, Kirker KR and Prestwich GD. Cross-linked hyaluronic acid hydrogel films: new biomaterials for drug delivery. $J$. Control. Release. 69 (1): 169-184 (2000).

[22] Gupta P, Vermani K and Garg S. Hydrogels: from controlled release to $\mathrm{pH}$-responsive drug delivery. Drug Discov. Today. 7(10): 569-579 (2002).

[23] Hamidi M, Azadi A and Rafiei P. Hydrogel nanoparticles in drug delivery. Adv. Drug Deliv. Rev. 60 (15): 1638-1649 (2008).

[24] Wang A, Cui Y, Li J and van Hest JCM. Fabrication of Gelatin Microgels by a 'Cast' Strategy for Controlled Drug Release. Adv. Funct. Mater. 22(13): 2673-2681 (2012).

[25] Anseth KS, Metters AT, Bryant SJ, Martens PJ, Elisseeff JH and Bowman CN. In situ forming degradable networks and their application in tissue engineering and drug delivery. J. Control. Release. 78: 199-209 (2002).

[26] Chung $C$ and Burdick JA. Influence of Three-Dimensional Hyaluronic Acid Microenvironments on Mesenchymal Stem Cell Chondrogenesis. Tissue Eng. Part A. 15(2): 243-254 (2009).

[27] Wang L-S, Chung JE, Chan PP-Y and Kurisawa M. Injectable biodegradable hydrogels with tunable mechanical properties for the stimulation of neurogenesic differentiation of human mesenchymal stem cells in 3D culture. Biomaterials. 31(6): 1148-1157 (2010).

[28] Crompton KE, Goud JD, Bellamkonda RV, Gengenbach TR, Finkelstein DI, Horne MK and Forsythe JS. Polylysinefunctionalised thermoresponsive chitosan hydrogel for neural tissue engineering. Biomaterials. 28(3): 441-449 (2007).

[29] Mohand-Kaci F, Assoul N, Martelly I, Allaire E and Zidi M. Optimized hyaluronic acid-hydrogel design and culture conditions for preservation of mesenchymal stem cell 
properties. Tissue Eng. Part C. Methods. 19(4): 288-298 (2013).

[30] Bryant SJ, Nicodemus GD and Villanueva I. Designing 3D photopolymer hydrogels to regulate biomechanical cues and tissue growth for cartilage tissue engineering. Pharm. Res. 25(10): 2379-2386 (2008).

[31] Fan C and Wang D-A. Effects of Permeability and Living Space on Cell Fate and Neo-Tissue Development in Hydrogel-Based Scaffolds: A Study With Cartilaginous Model. Macromol. Biosci.15(4): 535-545 (2014).

[32] Benoit DSW, Schwartz MP, Durney AR and Anseth KS. Small functional groups for controlled differentiation of hydrogelencapsulated human mesenchymal stem cells. Nat. Mater. 7(10): 816-823 (2008).

[33] Park KM, Shin YM, Joung YK, Shin $H$ and Park KD. In situ forming hydrogels based on tyramine conjugated 4-Arm-PPOPEO via enzymatic oxidative reaction. Biomacromolecules. 11(3): 706-712 (2010).

[34] Zhao W, Jin X, Cong Y, Liu Y and Fu J. Degradable natural polymer hydrogels for articular cartilage tissue engineering. J. Chem. Technol. Biotechnol. 88: 327-339 (2013).

[35] Hill-West JL, Chowdhury SM, Sawhney AS, Pathak CP, Dunn RC and Hubbell JA. Prevention of postoperative adhesions in the rat by in situ photopolymerization of bioresorbable hydrogel barriers. Obstet. Gynecol. 83(1): 59-64 (1994).

[36] Lieleg $\mathrm{O}$ and Ribbeck K. Biological Hydrogels as Selective Diffusion Barriers. Trends Cell Biol. 21(9): 543-551 (2011).

[37] West JL and Hubbell JA. Separation of the arterial wall from blood contact using hydrogel barriers reduces intimal thickening after balloon injury in the rat: The roles of medial 
and luminal factors in arterial healing. Proc. Natl. Acad. Sci. U. S. A. 93(23): 13188-13193 (1996).

[38] Hubbell JA. Hydrogel systems for barriers and local drug delivery in the control of wound healing. J. Control. Release. 39(2-3): 305-313 (1996).

[39] Dahlin C, Johansson A, Hoffman M and Molenberg A. Early biocompatibility of poly (ethylene glycol) hydrogel barrier materials for guided bone regeneration. An in vitro study using human gingival fibroblasts (HGF-1). Clin. Oral Implants Res. 25(1): 16-20 (2014).

[40] Van Vlierberghe S, Dubruel P and Schacht E. Biopolymerbased hydrogels as scaffolds for tissue engineering applications: a review. Biomacromolecules. 12(5): 1387-1408 (2011).

[41] Slaughter BV, Khurshid SS, Fisher OZ, Khademhosseini A and a Peppas N. Hydrogels in regenerative medicine. Adv. Mater. 21: 3307-3329 (2009).

[42] Ratner BD, Hoffman AS, Schoen FJ and Lemons JE. Biomaterials Science. 2nd ed. Elsevier Academic Press, 2004.

[43] Spiller KL, Maher SA and Lowman AM. Hydrogels for the Repair of Articular Cartilage Defects. Tissue Eng. Part B. 17(4): 281-299 (2011).

[44] Bryant SJ, Bender RJ, Durand KL and Anseth KS. Encapsulating chondrocytes in degrading PEG hydrogels with high modulus: Engineering gel structural changes to facilitate cartilaginous tissue production. Biotechnol. Bioeng. 86(7): 747-755 (2004).

[45] Bryant SJ and Anseth KS. Hydrogel properties influence ECM production by chondrocytes photoencapsulated in poly(ethylene glycol) hydrogels. J. Biomed. Mater. Res. 59(1): 63-72 (2002). 
[46] Wichterle $O$ and Lim D. Hydrophilic gels for biological use. Nature. 185: 117-118 (1960).

[47] Young C-D, Wu J-R and Tsou T-L. High-strength, ultra-thin and fiber-reinforced pHEMA artificial skin. Biomaterials 19 (19): 1745-1752 (1998).

[48] Ferreira L, Vidal MM and Gil MH. Evaluation of poly(2hydroxyethyl methacrylate) gels as drug delivery systems at different pH values. Int. J. Pharm. 194(2): 169-180 (2000).

[49] Zhang $Y$, Chu $D$, Zheng $M$, Kissel $T$ and Agarwal $S$. Biocompatible and degradable poly(2-hydroxyethyl methacrylate) based polymers for biomedical applications. Polym. Chem. 3(10): 2752-2759 (2012).

[50] Nguyen QT, Hwang $\mathrm{Y}$, Chen AC, Varghese $\mathrm{S}$ and Sah RL. Cartilage-like mechanical properties of poly (ethylene glycol)diacrylate hydrogels. Biomaterials. 33(28): 6682-6690 (2012).

[51] Nolan CM, Reyes CD, Debord JD, García AJ and Lyon LA. Phase transition behavior, protein adsorption, and cell adhesion resistance of poly(ethylene glycol) cross-linked microgel particles. Biomacromolecules. 6(4): 2032-2039 (2005).

[52] Rizzi SC and a Hubbell JA. Recombinant protein-co-PEG networks as cell-adhesive and proteolytically degradable hydrogel matrixes. Part I: Development and physicochemical characteristics. Biomacromolecules. 6(3): 1226-1238 (2005).

[53] Yu F, Cao X, Li Y, Zeng L, Yuan B and Chen X. An injectable hyaluronic acid/PEG hydrogel for cartilage tissue engineering formed by integrating enzymatic crosslinking and Diels-Alder 'click chemistry. Polym. Chem. 5(3): 1082-1090 (2014).

[54] Ferretti M, Marra KG, Kobayashi K, Defail AJ and Chu CR. Controlled in vivo degradation of genipin crosslinked polyethylene glycol hydrogels within osteochondral defects. Tissue Eng. 12(9): 2657-2663 (2006). 
[55] Bosnakovski D, Mizuno M, Kim G, Takagi S,Okumura M and Fujinaga T. Chondrogenic differentiation of bovine bone marrow mesenchymal stem cells (MSCs) in different hydrogels: influence of collagen type II extracellular matrix on MSC chondrogenesis. Biotechnol. Bioeng. 93(6): 1152-1163 (2006).

[56] Nöth U, Rackwitz L, Heymer A, Weber M, Baumann B, Steinert $A$, Schütze N, Jakob $F$ and Eulert J. Chondrogenic differentiation of human mesenchymal stem cells in collagen type I hydrogels. J. Biomed. Mater. Res. A. 83(3): 626-635 (2007).

[57] Zhang L, Yuan T, Guo L and Zhang X. An in vitro study of collagen hydrogel to induce the chondrogenic differentiation of mesenchymal stem cells. J Biomed Mater Res Part A. 100A: 2717-2725 (2012).

[58] Zheng L, Fan HS, Sun J, Chen XN, Wang G, Zhang L, Fan YJ and Zhang XD. Chondrogenic differentiation of mesenchymal stem cells induced by collagen-based hydrogel: an in vivo study. J. Biomed. Mater. Res. A. 93A(2): 783-792 (2010).

[59] Du M, Liang H, Mou C, Li X, Sun J, Zhuang Y, Xiao Z, Chen B and Dai J. Regulation of human mesenchymal stem cells differentiation into chondrocytes in extracellular matrix-based hydrogel scaffolds. Colloids Surf. B. Biointerfaces. 114: 316323 (2014).

[60] Lee JE, Kim KE, Kwon IC, Ahn HJ, Lee S-H, Cho H, Kim HJ, Seong SC and Lee MC. Effects of the controlled-released TGFbeta 1 from chitosan microspheres on chondrocytes cultured in a collagen/chitosan/glycosaminoglycan scaffold. Biomaterials. 25(18): 4163-4173 (2004).

[61] Zhu C, Fan D, Duan Z, Xue W, Shang L, Chen F and Luo Y. Initial investigation of novel human-like collagen/chitosan scaffold for vascular tissue engineering. J. Biomed. Mater. Res. A. 89(3): 829-840 (2009). 
[62] Suri S and Schmidt CE. Photopatterned collagen-hyaluronic acid interpenetrating polymer network hydrogels. Acta Biomater. 5(7): 2385-2397 (2009).

[63] Tang S, Vickers SM, Hsu H-P and Spector M. Fabrication and characterization of porous hyaluronic acid-collagen composite scaffolds. J. Biomed. Mater. Res. A. 82(2): 323-335 (2007).

[64] Liao E, Yaszemski M, Krebsbach $P$ and Hollister S. Tissueengineered cartilage constructs using composite hyaluronic acid/collagen I hydrogels and designed poly(propylene fumarate) scaffolds. Tissue Eng. 13(3): 537-550 (2007).

[65] Cheng Y, Lu J, Liu S, Zhao P, Lu G and Chen J. The preparation, characterization and evaluation of regenerated cellulose/collagen composite hydrogel films. Carbohydr. Polym. 107: 57-64 (2014).

[66] Ko C-S, Huang J-P, Huang C-W and Chu IM. Type II collagenchondroitin sulfate-hyaluronan scaffold cross-linked by genipin for cartilage tissue engineering. J. Biosci. Bioeng. 107(2): 177-182 (2009).

[67] Kuijpers AJ, Engbers GHM, Meyvis TKL, de Smedt SSC, Demeester J, Krijgsveld J, Zaat SAJ, Dankert J and Feijen J. Combined Gelatin-Chondroitin Sulfate Hydrogels for Controlled Release of Cationic Antibacterial Proteins. Macromolecules. 33(10): 3705-3713 (2000).

[68] Brown KE, Leong K, Huang CH, Dalal R, Green GD, Haimes HB, Jimenez PA and Bathon J. Gelatin/chondroitin 6-sulfate microspheres for the delivery of therapeutic proteins to the joint. Arthritis Rheum. 41(12): 2185-2195 (1998).

[69] Shu XZ, Liu Y, Palumbo $F$ and Prestwich GD. Disulfidecrosslinked hyaluronan-gelatin hydrogel films: a covalent mimic of the extracellular matrix for in vitro cell growth. Biomaterials. 24(21): 3825-3834 (2003). 
[70] Huang $X$, Zhang $Y$, Zhang $X, X u L$, Chen $X$ and Wei S. Influence of radiation crosslinked carboxymethyl-chitosan/gelatin hydrogel on cutaneous wound healing. Mater. Sci. Eng. C. 33(8): 4816-4824 (2013).

[71] Jiankang $H$, Dichen L, Yaxiong L, Bo $Y$, Hanxiang $Z$, Qin L, Bingheng $L$ and $Y i$ L. Preparation of chitosan-gelatin hybrid scaffolds with well-organized microstructures for hepatic tissue engineering. Acta Biomater. 5(1): 453-461 (2009).

[72] Haroun AA, Gamal-Eldeen A and Harding DRK. Preparation, characterization and in vitro biological study of biomimetic three-dimensional gelatin-montmorillonite/cellulose scaffold for tissue engineering. J. Mater. Sci. Mater. Med. 20(12): 2527-2540 (2009).

[73] Sakai S, Hashimoto I and Kawakami K. Synthesis of an agarosegelatin conjugate for use as a tissue engineering scaffold. $J$. Biosci. Bioeng. 103(1): 22-26 (2007).

[74] Li Y, Jia H, Cheng Q, Pan F and Jiang Z. Sodium alginate-gelatin polyelectrolyte complex membranes with both high water vapor permeance and high permselectivity. J. Memb. Sci. 375(1-2): 304-312 (2011).

[75] Wang T-W, Sun J-S, Wu H-C, Tsuang $\mathrm{Y}-\mathrm{H}$, Wang $\mathrm{W}-\mathrm{H}$ and Lin F$\mathrm{H}$. The effect of gelatin-chondroitin sulfate-hyaluronic acid skin substitute on wound healing in SCID mice. Biomaterials. 27(33): 5689-5697 (2006).

[76] Chang $\mathrm{C}-\mathrm{H}$, Kuo T-F, Lin C-C, Chou C-H, Chen K-H, Lin F-H and Liu H-C. Tissue engineering-based cartilage repair with allogenous chondrocytes and gelatin-chondroitin-hyaluronan tri-copolymer scaffold: a porcine model assessed at 18, 24, and 36 weeks. Biomaterials. 27(9): 1876-1888 (2006).

[77] Yang S-H, Chen P-Q, Chen Y-F and Lin F-H. An In-vitro Study on Regeneration of Human Nucleus Pulposus by Using 
Gelatin/Chondroitin-6-Sulfate/Hyaluronan Tri-copolymer

Scaffold. Artif. Organs. 29(10): 806-814 (2005).

[78] Chang C-H, Liu H-C, Lin C-C, Chou C-H and Lin F-H. Gelatinchondroitin-hyaluronan tri-copolymer scaffold for cartilage tissue engineering. Biomaterials. 24(26): 4853-4858 (2003).

[79] Tan H, Wu J, Lao L and Gao C. Gelatin/chitosan/hyaluronan scaffold integrated with PLGA microspheres for cartilage tissue engineering. Acta Biomater. 5(1): 328-337 (2009).

[80] Bhat S and Kumar A. Cell proliferation on three-dimensional chitosan-agarose-gelatin cryogel scaffolds for tissue engineering applications. J. Biosci. Bioeng. 114(6): 663-670 (2012).

[81] Park S-H, Cui JH, Park SR and Min B-H. Potential of fortified fibrin/hyaluronic acid composite gel as a cell delivery vehicle for chondrocytes. Artif. Organs. 33(6): 439-447 (2009).

[82] Pereira RC, Scaranari M, Castagnola P, Grandizio M, Azevedo HS, Reis RL, Cancedda R and Gentili G. Novel injectable gel (system) as a vehicle for human articular chondrocytes in cartilage tissue regeneration. J. Tissue Eng. Regen. Med. 3: 97106 (2009).

[83] Gamboa-Martínez TC, García Cruz DM, Carda C, Ribelles JLG and Ferrer GG. Fibrin-chitosan composite substrate for in vitro culture of chondrocytes. J. Biomed. Mater. Res. A. 101(2): 404-412 (2013).

[84] She Z, Zhang B, Jin C, Feng Q and Xu Y. Preparation and in vitro degradation of porous three-dimensional silk fibroin/chitosan scaffold. Polym. Degrad. Stab. 93(7): 1316-1322 (2008).

[85] Bhardwaj N, Nguyen QT, Chen AC, Kaplan DL, Sah RL and S. C. Kundu. Potential of 3-D tissue constructs engineered from bovine chondrocytes/silk fibroin-chitosan for in vitro cartilage tissue engineering. Biomaterials. 32(25): 5773-5781 (2011). 
[86] She Z, Jin C, Huang Z, Zhang B, Feng $Q$ and Xu Y. Silk fibroin/chitosan scaffold: preparation, characterization, and culture with HepG2 cell. J. Mater. Sci. Mater. Med. 19(12): 3545-3553 (2008).

[87] Garcia-Fuentes $M$, Meinel AJ, Hilbe $M$, Meinel $L$ and Merkle HP. Silk fibroin/hyaluronan scaffolds for human mesenchymal stem cell culture in tissue engineering. Biomaterials. 30(28): 5068-5076 (2009).

[88] Ren Y-J, Zhou Z-Y, Liu B-F, Xu Q-Y and Cui F-Z. Preparation and characterization of fibroin/hyaluronic acid composite scaffold. Int. J. Biol. Macromol. 44(4): 372-378 (2009).

[89] Schrieber R and Gareis H. Gelatine Handbook. Theory and Industrial Practice. Eberbach (Germany): Wiley-VCH, 2007.

[90] Wang L-S, Du C, Chung JE and Kurisawa M. Enzymatically cross-linked gelatin-phenol hydrogels with a broader stiffness range for osteogenic differentiation of human mesenchymal stem cells. Acta Biomater. 8(5): 1826-1837 (2012).

[91] Choi YS, Hong SR, Lee YM, Song KW, Park MH and Nam YS. Studies on gelatin-containing artificial skin: II. Preparation and characterization of cross-linked gelatin-hyaluronate sponge. J. Biomed. Mater. Res. 48(5): 631-639 (1999).

[92] Zhou Z, Chen J, Peng C, Huang T, Zhou H, Ou B, Chen J, Liu Q, He S, Cao D, Huang $\mathrm{H}$ and Xiang L. Fabrication and Physical Properties of Gelatin/Sodium Alginate/Hyaluronic Acid Composite Wound Dressing Hydrogel. J. Macromol. Sci. Part A. 51(4): 318-325 (2014).

[93] Lee Y, Bae JW, Oh DH, Park KM, Chun YW, Sung H-J and Park KD. In situ forming gelatin-based tissue adhesives and their phenolic content-driven properties. J. Mater. Chem. B. 1(18): 2407-2414 (2013). 
[94] Liu Y, Ren L and Wang Y. Crosslinked collagen-gelatinhyaluronic acid biomimetic film for cornea tissue engineering applications. Mater. Sci. Eng. C. 33(1): 196-201 (2013).

[95] Taubenberger AV, Woodruff MA, Bai H, Muller DJ and Hutmacher DW. The effect of unlocking RGD-motifs in collagen I on pre-osteoblast adhesion and differentiation. Biomaterials. 31(10): 2827-2835 (2010).

[96] da Silva MA, Bode F, Grillo I and Dreiss CA. Exploring the Kinetics of Gelation and Final Architecture of Enzymatically Cross-Linked Chitosan/Gelatin Gels. Biomacromolecules. 16(4): 1401-1409 (2015).

[97] Bigi A. Drawn gelatin films with improved mechanical properties. Biomaterials. 19(24): 2335-2340 (1998).

[98] Olde Damink LHH, Dijkstra PJ, Van Luyn MJA, Van Wachem PB, Nieuwenhuis $P$ and Feijen J. Glutaraldehyde as a crosslinking agent for collagen-based biomaterials. J. Mater. Sci. Mater. Med. 6(8): 460-472 (1995).

[99] Xing Q, Yates K, Vogt C, Qian Z, Frost MC and Zhao F. Increasing Mechanical Strength of Gelatin Hydrogels by Divalent Metal Ion Removal. Sci. Rep. 4: 4706 (2014).

[100] Chen K-Y, Dong G-C, Hsu C-Y, Chen Y-S and Yao C-H. Autologous bone marrow stromal cells loaded onto porous gelatin scaffolds containing Drynaria fortunei extract for bone repair. J. Biomed. Mater. Res. A. 101(4): 954-962 (2013).

[101] Liang $\mathrm{H}-\mathrm{C}$, Chang $\mathrm{W}-\mathrm{H}$, Liang $\mathrm{H}-\mathrm{F}$, Lee $\mathrm{M}-\mathrm{H}$ and Sung $\mathrm{H}-\mathrm{W}$. Crosslinking structures of gelatin hydrogels crosslinked with genipin or a water-soluble carbodiimide. J. Appl. Polym. Sci. 91(6): 4017-4026 (2004).

[102] Girish KS and Kemparaju K. The magic glue hyaluronan and its eraser hyaluronidase: a biological overview. Life Sci. 80(21): 1921-1943 (2007). 
[103] Balazs EA and Band PA. Therapeutic Use of Hyaluronan-Based Products. Carbohydrate Chemistry, Biology and Medical Applications, Elsevier, 2008, pp. 311-332.

[104] Turner NJ, Kielty CM, Walker MG and Canfield AE. A novel hyaluronan-based biomaterial (Hyaff-11) as a scaffold for endothelial cells in tissue engineered vascular grafts. Biomaterials. 25(28): 5955-5964 (2004).

[105] Collins MN and Birkinshaw C. Comparison of the Effectiveness of Four Different Crosslinking Agents with Hyaluronic Acid Hydrogel Films for Tissue-Culture Applications J. Appl. Polym. Sci. 104: 3183-3191 (2007).

[106] Kim KS, Park SJ, Yang J, Jeon J-H, Bhang SH, Kim B-S and Hahn SK. Injectable hyaluronic acid-tyramine hydrogels for the treatment of rheumatoid arthritis. Acta Biomater. 7(2): 666674 (2011).

[107] Li Y, Rodrigues J and Tomás $\mathrm{H}$. Injectable and biodegradable hydrogels: gelation, biodegradation and biomedical applications. Chem. Soc. Rev. 41(6): 2193-2221 (2012).

[108] Kim IL, Mauck RL and Burdick JA. Hydrogel design for cartilage tissue engineering: a case study with hyaluronic acid. Biomaterials. 32(34): 8771-8782 (2011).

[109] Erickson IE, Kestle SR, Zellars KH, Farrell MJ, Kim M, Burdick JA and Mauck RL. High mesenchymal stem cell seeding densities in hyaluronic acid hydrogels produce engineered cartilage with native tissue properties. Acta Biomater. 8(8): 3027-3034 (2012).

[110] Chung C, Beecham M, Mauck RL and Burdick JA. The influence of degradation characteristics of hyaluronic acid hydrogels on in vitro neocartilage formation by mesenchymal stem cells. Biomaterials. 30(26): 4287-4296 (2009). 
[111] Toh WS, Lim TC, Kurisawa M and Spector M. Modulation of mesenchymal stem cell chondrogenesis in a tunable hyaluronic acid hydrogel microenvironment. Biomaterials. 33: 3835-3845 (2012).

[112] Zhu M, Feng Q and Bian L. Differential effect of hypoxia on human mesenchymal stem cell chondrogenesis and hypertrophy in hyaluronic acid hydrogels. Acta Biomater. 10(3): 1333-1340 (2014).

[113] Snyder TN, Madhavan K, Intrator M, Dregalla RC and Park D. A fibrin/hyaluronic acid hydrogel for the delivery of mesenchymal stem cells and potential for articular cartilage repair. J. Biol. Eng. 8: 10 (2014).

[114] Segura T, Anderson BC, Chung PH, Webber RE, Shull KR and Shea LD. Crosslinked hyaluronic acid hydrogels: a strategy to functionalize and pattern. Biomaterials. 26(4): 359-371 (2005).

[115] Wang T-W, Wu H-C, Huang Y-C, Sun J-S and Lin F-H. Biomimetic Bilayered Gelatin-Chondroitin 6 Sulfate-Hyaluronic Acid Biopolymer as a Scaffold for Skin Equivalent Tissue Engineering. Artif. Organs 30(3): 141-149 (2006).

[116] Tan H, Chu CR, Payne KA and Marra KG. Injectable in situ forming biodegradable chitosan-hyaluronic acid based hydrogels for cartilage tissue engineering. Biomaterials. 30(13): 2499-2506 (2009).

[117] Shu XZ, Liu Y, Palumbo $F$ and Prestwich GD. Disulfidecrosslinked hyaluronan-gelatin hydrogel films: a covalent mimic of the extracellular matrix for in vitro cell growth. Biomaterials. 24(21): 3825-3834 (2003).

[118] Weng L, Pan H and Chen W. Self-crosslinkable hydrogels composed of partially oxidized hyaluronan and gelatin: In vitro and in vivo responses J. Biomed. Mater. Res. Part A. 85A(2): 352-365 (2008). 
[119] Chen Y-C, Su W-Y, Yang S-H, Gefen A and Lin F-H. In situ forming hydrogels composed of oxidized high molecular weight hyaluronic acid and gelatin for nucleus pulposus regeneration. Acta Biomater. 9(2): 5181-5193 (2013).

[120] Zhou Z, Yang Z, Kong L, Liu L, Liu Q, Zhao Y, Zeng W, Yi Q and Cao D. Preparation and Characterization of Hyaluronic Acid Hydrogel Blends with Gelatin. J. Macromol. Sci. Part B. 51(12): 2392-2400 (2012).

[121] Vanderhooft JL, Alcoutlabi M, Magda JJ and Prestwich GD. Rheological properties of cross-linked hyaluronan-gelatin hydrogels for tissue engineering. Macromol. Biosci. 9(1): 2028 (2009).

[122] Camci-Unal G, Cuttica D, Annabi N, Demarchi D and Khademhosseini A. Synthesis and Characterization of Hybrid Hyaluronic Acid-Gelatin Hydrogels. Biomacromolecules. 14 (4): 1085-1092 (2013).

[123] Levett PA, Hutmacher DW, Malda J and Klein TJ. Hyaluronic Acid Enhances the Mechanical Properties of TissueEngineered Cartilage Constructs. PLoS One. 9(12): e113216 (2014).

[124] Lai J-Y. Biocompatibility of chemically cross-linked gelatin hydrogels for ophthalmic use. J. Mater. Sci. Mater. Med. 21: 1899-1911 (2010).

[125] Jun I, Kim SJ, Choi E, Park KM, Rhim T, Park J, Park KD and Shin $\mathrm{H}$. Preparation of Biomimetic Hydrogels with Controlled Cell Adhesive Properties and Topographical Features for the Study of Muscle Cell Adhesion and Proliferation. Macromol. Biosci. 12(11): 1502-1513 (2012).

[126] Van Tomme SR, Storm G and Hennink WE. In situ gelling hydrogels for pharmaceutical and biomedical applications. Int. J. Pharm. 355: 1-18 (2008). 
[127] Bae KH, Wang L-S and Kurisawa M. Injectable biodegradable hydrogels: progress and challenges. J. Mater. Chem. B. 1: 5371 (2013).

[128] Teixeira LSM, Feijen J, van Blitterswijk CA, Dijkstra PJ and Karperien M. Enzyme-catalyzed crosslinkable hydrogels: emerging strategies for tissue engineering. Biomaterials. 33: 1281-1290 (2012).

[129] Sakai S, Hirose K, Taguchi K, Ogushi Y and Kawakami K. An injectable, in situ enzymatically gellable, gelatin derivative for drug delivery and tissue engineering. Biomaterials. 30: 33713377 (2009).

[130] Jun I, Park KM, Lee DY, Park KD and Shin H. Control of adhesion, focal adhesion assembly, and differentiation of myoblasts by enzymatically crosslinked cell-interactive hydrogels. Macromol. Res. 19(9): 911-920 (2011).

[131] Kurisawa M, Chung JE, Yang YY, Gao SJ and Uyama $H$. Injectable biodegradable hydrogels composed of hyaluronic acid-tyramine conjugates for drug delivery and tissue engineering. Chem. Commun. (Camb). 34: 4312-4314 (2005).

[132] Jin R, Hiemstra C, Zhong Z and Feijen J. Enzyme-mediated fast in situ formation of hydrogels from dextran-tyramine conjugates. Biomaterials. 28(18): 2791-2800 (2007).

[133] Nakajima $\mathrm{N}$ and Ikada Y. Mechanism of Amide Formation by Carbodiimide for Bioconjugation in Aqueous Media. Bioconjug. Chem. 6(1): 123-130 (1995).

[134] Lee F, Chung JE and Kurisawa M. An injectable hyaluronic acid-tyramine hydrogel system for protein delivery. J. Control. Release. 134: 186-193 (2009).

[135] Sakai S, Moriyama K, Taguchi K and Kawakami K. Hematin is an alternative catalyst to horseradish peroxidase for in situ 
hydrogelation of polymers with phenolic hydroxyl groups in vivo. Biomacromolecules. 11(8): 2179-2183 (2010).

[136] Lee F, Chung JE and Kurisawa M. An injectable enzymatically crosslinked hyaluronic acid-tyramine hydrogel system with independent tuning of mechanical strength and gelation rate. Soft Matter. 4: 880-887 (2008).

[137] Yung CW, Wu LQ, Tullman JA, Payne GF, Bentley WE and Barbari TA. Transglutaminase crosslinked gelatin as a tissue engineering scaffold. J. Biomed. Mater. Res. A. 83(4): 10391046, (2007).

[138] Chen T, Embree HD, Brown EM, Taylor MM and Payne GF. Enzyme-catalyzed gel formation of gelatin and chitosan: potential for in situ applications. Biomaterials. 24: 2831-2841 (2003).

[139] Moreira Teixeira LS, Bijl S, Pully VV, Otto C, Jin R, Feijen J, van Blitterswijk CA, Dijkstra PJ and Karperien M. Self-attaching and cell-attracting in-situ forming dextran-tyramine conjugates hydrogels for arthroscopic cartilage repair. Biomaterials. 33(11): 3164-3174 (2012).

[140] Wang R, Leber N, Buhl C, Verdonschot N, Dijkstra PJ and Karperien M. Cartilage adhesive and mechanical properties of enzymatically crosslinked polysaccharide tyramine conjugate hydrogels. Polym. Adv. Technol. 25(5): 568-574 (2014).

[141] Chuang C-H, Lin R-Z, Tien H-W, Chu Y-C, Li Y-C, Melero-Martin $\mathrm{JM}$ and Chen Y-C. Enzymatic regulation of functional vascular networks using gelatin hydrogels. Acta Biomater. 19: 85-99 (2015).

[142] Gu WY, Yao H, Huang CY and Cheung HS. New insight into deformation-dependent hydraulic permeability of gels and cartilage, and dynamic behavior of agarose gels in confined compression. J. Biomech. 36: 593-598 (2003). 
[143] Cha C, Soman P, Zhu W, Nikkhah M, Camci-Unal G, Chen S and Khademhosseini A. Structural Reinforcement of Cell-Laden Hydrogels with Microfabricated Three Dimensional Scaffolds. Biomater. Sci. 2(5): 703-709 (2014).

[144] Myung D, Waters D, Wiseman M, Duhamel P-E, Noolandi J, Ta $\mathrm{CN}$ and Frank CW. Progress in the development of interpenetrating polymer network hydrogels. Polym. Adv. Technol. 19(6): 647-657 (2008).

[145] Spagnol C, Rodrigues FHA, Neto AGVC, Pereira AGB, Fajardo AR, Radovanovic E, Rubira AF and Muniz EC. Nanocomposites based on poly(acrylamide-co-acrylate) and cellulose nanowhiskers. Eur. Polym. J. 48(3): 454-463 (2012).

[146] Moutos FT and Guilak F. Composite scaffolds for cartilage tissue engineering. Biorheology. 45(3-4): 501-512 (2008).

[147] Liu X, Huang C, Feng Y, Liang J, Fan Y, Gu Z and Zhang X. Reinforcement of a Porous Collagen Scaffold with SurfaceActivated PLA Fibers. J. Biomater. Sci. 21: 963-977 (2010).

[148] Wayne JS, McDowell CL, Shields KJ and Tuan RS. In vivo response of polylactic acid-alginate scaffolds and bone marrow-derived cells for cartilage tissue engineering. Tissue Eng. 11(5-6): 953-963 (2005).

[149] Sherwood JK, Riley SL, Palazzolo R, Brown SC, Monkhouse DC, Coates M, Griffith LG, Landeen LK and Ratcliffe A. A threedimensional osteochondral composite scaffold for articular cartilage repair. Biomaterials. 23(24): 4739-4751 (2002).

[150] Regev O, Reddy CS, Nseir N and Zussman E. Hydrogel Reinforced by Short Albumin Fibers: Mechanical Characterization and Assessment of Biocompatibility. Macromol. Mater. Eng. 298: 283-291 (2013). 
[151] Moutos FT, Freed LE and Guilak F. A biomimetic threedimensional woven composite scaffold for functional tissue engineering of cartilage. Nat. Mater. 6: 162-167 (2007).

[152] Maranchi JP, Trexler MM, Guo Q and Elisseeff JH. Fibrereinforced hydrogels with high optical transparency. Int. Mater. Rev. 59(5): 264-296 (2014).

[153] Hardy JG, Lin P and Schmidt CE. Biodegradable hydrogels composed of oxime crosslinked poly(ethylene glycol), hyaluronic acid and collagen: a tunable platform for soft tissue engineering. J. Biomater. Sci. Polym. Ed. 26(3): 143-161 (2015).

[154] Feng $Y$, Zhao H, Zhang S, Jiao L, Lu J, Wang $\mathrm{H}$ and Guo J. Polyester-Hydrophilic PEO Networks as Multifunctional Biomaterials. Macromol. Symp. 306-307(1): 18-26 (2011).

[155] Visser J, Melchels FPW, Jeon JE, van Bussel EM, Kimpton LS, Byrne HM, Dhert WJA, Dalton PD, Hutmacher DW and Malda J. Reinforcement of hydrogels using three-dimensionally printed microfibres. Nat. Commun. 6: 6933 (2015).

[156] Dragan ES, Perju MM and Dinu MV. Preparation and characterization of IPN composite hydrogels based on polyacrylamide and chitosan and their interaction with ionic dyes. Carbohydr. Polym. 88(1): 270-281 (2012).

[157] Gong JP, Katsuyama Y, Kurokawa T and Osada Y. DoubleNetwork Hydrogels with Extremely High Mechanical Strength. Adv. Mater. 15(14): 1155-1158 (2003).

[158] Dragan ES. Design and applications of interpenetrating polymer network hydrogels. A review. Chem. Eng. J. 243: 572590 (2014).

[159] Parizel N, Meyer G and Weill G. Morphologies of semi and full interpenetrating polymer networks by nuclear magnetic 
resonance relaxation times. Polymer. 36(12): 2323-2330 (1995).

[160] Salmerón Sánchez M, Gallego Ferrer G, Torregrosa Cabanilles $C$, Meseguer Dueñas JM, Monleón Pradas M and Gómez Ribelles JL. Forced compatibility in poly (methyl acrylate)/ poly (methyl methacrylate) sequential interpenetrating polymer networks. Polymer. 42: 10071-10075 (2001).

[161] Eichhorn SJ, Dufresne A, Aranguren $M$, Marcovich NE, Capadona JR, Rowan SJ, Weder C, Thielemans W, Roman M, Renneckar S, Gindl W, Veigel S, Keckes J, Yano H, Abe K, Nogi M, Nakagaito AN, Mangalam A, Simonsen J, Benight AS, Bismarck A, Berglund LA and Peijs T. Review: current international research into cellulose nanofibres and nanocomposites. J Mater Sci.45(1): 1-33 (2010).

[162] Herrera-Franco PJ and Valadez-González A. A study of the mechanical properties of short natural-fiber reinforced composites. Compos. Part B Eng. 36(8): 597-608 (2005).

[163] Abdelmouleh M, Boufi S, Belgacem M and Dufresne A. Short natural-fibre reinforced polyethylene and natural rubber composites: Effect of silane coupling agents and fibres loading. Compos. Sci. Technol. 67(7-8): 1627-1639 (2007).

[164] Sutti A, Lin $T$ and Wang $X$. Shear-Enhanced Solution Precipitation: A Simple Process to Produce Short Polymeric Nanofibers. J. Nanosci. Nanotechnol. 11(10): 8947-8952 (2011).

[165] de Moraes MA, Paternotte E, Mantovani D and Beppu MM. Mechanical and biological performances of new scaffolds made of collagen hydrogels and fibroin microfibers for vascular tissue engineering. Macromol. Biosci. 12(9): 12531264 (2012).

[166] Chen $W, Y$ Yu H, Liu $Y$, Chen $P$, Zhang $M$ and Hai $Y$. Individualization of cellulose nanofibers from wood using 
high-intensity ultrasonication combined with chemical pretreatments. Carbohydr. Polym. 83(4): 1804-1811 (2011).

[167] Greenfeld I and Zussman E. Polymer entanglement loss in extensional flow: Evidence from electrospun short nanofibers. J. Polym. Sci. Part B Polym. Phys. 51(18): 1377-1391 (2013).

[168] Coburn JM, Gibson M, Monagle S, Patterson Z and Elisseeff JH. Bioinspired nano fibers support chondrogenesis for articular cartilage repair. PNAS. 109(25): 10012-10017 (2012).

[169] Palma Santana B, Dos Reis Paganotto GF, Nedel F, Piva E, Varella de Carvalho R, Nör JE, Demarco FF and Villarreal Carreño NL. Nano-/microfiber scaffold for tissue engineering: physical and biological properties. J. Biomed. Mater. Res. A. 100A: 3051-3058 (2012).

[170] Hsieh A, Zahir T, Lapitsky Y, Amsden B, Wan W and Shoichet MS. Hydrogel/electrospun fiber composites influence neural stem/progenitor cell fate. Soft Matter. 6(10): 2227-2237 (2010).

[171] Facca AG, Kortschot MT and Yan N. Predicting the elastic modulus of natural fibre reinforced thermoplastics. Compos. Part A Appl. Sci. Manuf. 37(10): 1660-1671 (2006).

[172] Tucker III CL and Liang E. Stiffness predictions for unidirectional short-fiber composites: Review and evaluation. Compos. Sci. Technol. 59(5): 655-671 (1999).

[173] Yan S, Xiaoqiang L, Shuiping L, Hongsheng W and Chuanglong $\mathrm{H}$. Fabrication and properties of PLLA-gelatin nanofibers by electrospinning. J. Appl. Polym. Sci. 117: 542-547 (2010).

[174] Plazas Bonilla CE and Perilla JE. The past, present and near future of materials for use in biodegradable orthopaedic implants. Ing. e Investig. 31(2): 124-133 (2011). 
[175] Van Beek $M$, Jones $L$ and Sheardown H. Hyaluronic acid containing hydrogels for the reduction of protein adsorption. Biomaterials. 29(7): 780-789 (2008).

[176] Johnson RP, Jeong Y-I, Choi E, Chung C-W, Kang DH, Oh S-O, Suh $H$ and Kim I. Biocompatible Poly(2-hydroxyethyl methacrylate)-b-poly(L-histidine) Hybrid Materials for $\mathrm{pH}$ Sensitive Intracellular Anticancer Drug Delivery. Adv. Funct. Mater. 22(5): 1058-1068 (2012).

[177] Babu M, Yadav HKS, Moin A and Shivakumar HG. In vitro-in vivo evaluation of poly(2-hydroxyethyl methacrylate-comethyl methacrylate) hydrogel implants containing cisplatin. Acta Pharm. Sin. B. 1(4): 261-267 (2011).

[178] Modi S, Koelling K and Vodovotz Y. Assessing the mechanical, phase inversion, and rheological properties of poly-[(R)-3hydroxybutyrate-co-(R)-3-hydroxyvalerate] (PHBV) blended with poly-(I-lactic acid) (PLA). Eur. Polym. J. 49(11): 3681-3690 (2013).

[179] Kai D, Prabhakaran MP, Stahl B, Eblenkamp M, Wintermantel $\mathrm{E}$ and Ramakrishna S. Mechanical properties and in vitro behavior of nanofiber-hydrogel composites for tissue engineering applications. Nanotechnology. 23: 095705 (10pp) (2012).

[180] Montaño-Leyva B, da Silva GGD, Gastaldi E, Torres-Chávez P, Gontard $\mathrm{N}$ and Angellier-Coussy $\mathrm{H}$. Biocomposites from wheat proteins and fibers: Structure/mechanical properties relationships. Ind. Crops Prod. 43: 545-555 (2013).

[181] Coburn J, Gibson M, Bandalini PA, Laird C, Mao H-Q, Moroni L, Seliktar D and Elisseeff J. Biomimetics of the extracellullar matrix: an integrated three dimensional fiber-hydrogel composite for cartilage tissue engineering. Smart Struct. Syst. 7(3): 213-222 (2011). 
[182] Tonsomboon K and Oyen ML. Composite electrospun gelatin fiber-alginate gel scaffolds for mechanically robust tissue engineered cornea. J. Mech. Behav. Biomed. Mater. 21: 185194 (2013).

[183] Brigham MD, Bick A, Lo E, Bendali A, Burdick JA and Khademhosseini $A$. Mechanically robust and bioadhesive collagen and photocrosslinkable hyaluronic acid semiinterpenetrating networks. Tissue Eng. Part A. 15(7): 16451653 (2009).

[184] Mehra TD, Ghash K, Shu XZ, Prestwich GD and Clark RAF. Molecular stenting with a crosslinked hyaluronan derivative inhibits collagen gel contraction. J Invest Dermatol. 126: 2202 (2006).

[185] Yamato M, Adachi E, Yamamoto K and Hayashi T. Condensation of collagen fibrils to the direct vicinity of fibroblasts as a cause of gel contraction. J Biochem. 117: 940 (1995).

[186] Fu Y, Xu K, Zheng X, Giacomin AJ, Mix AW and Kao WJ. 3D cell entrapment in crosslinked thiolated gelatin-poly(ethylene glycol) diacrylate hydrogels. Biomaterials. 33(1): 48-58 (2012).

[187] Baker BM and Chen CS. Deconstructing the third dimension: how 3D culture microenvironments alter cellular cues. J. Cell Sci. 125(13): 3015-3024 (2012).

[188] Steward AJ, Wagner DR and Kelly DJ. The pericellular environment regulates cytoskeletal development and the differentiation of mesenchymal stem cells and determines their response to hydrostatic pressure. Eur. Cell. Mater. 25: 167-178 (2013).

[189] Wang L-S, Du C, Chung JE and Kurisawa M. Enzymatically cross-linked gelatin-phenol hydrogels with a broader stiffness range for osteogenic differentiation of human mesenchymal stem cells. Acta Biomater. 8(5): 1826-1837 (2012). 
[190] Wang L-S, Du C, Toh WS, Wan ACA, Gao SJ and Kurisawa M. Modulation of chondrocyte functions and stiffness-dependent cartilage repair using an injectable enzymatically crosslinked hydrogel with tunable mechanical properties. Biomaterials. 35(7): 2207-2217 (2014).

[191] Lai J-Y. Corneal Stromal Cell Growth on Gelatin/Chondroitin Sulfate Scaffolds Modified at Different NHS/EDC Molar Ratios. Int. J. Mol. Sci. 14: 2036-2055 (2013).

[192] Edlund U, Källrot M and Albertsson A-C. Single-step covalent functionalization of polylactide surfaces. J. Am. Chem. Soc. 127(24): 8865-8871 (2005).

[193] Källrot M, Edlund U and Albertsson A-C. Covalent grafting of poly(L-lactide) to tune the in vitro degradation rate. Biomacromolecules. 8(8): 2492-2496 (2007).

[194] Ma H, Davis RH and Bowman CN. A Novel Sequential Photoinduced Living Graft Polymerization. Macromolecules. 33(2): 331-335 (2000).

[195] Janorkar AV, Metters AT and Hirt DE. Modification of Poly(lactic acid) Films: Enhanced Wettability from SurfaceConfined Photografting and Increased Degradation Rate Due to an Artifact of the Photografting Process. Macromolecules. 37(24): 9151-9159 (2004).

[196] Nugroho RWN, Odelius K, Höglund A and Albertsson A-C. Nondestructive Covalent 'Grafting-from' of Poly(lactide) Particles of Different Geometries. ACS Appl. Mater. Interfaces. 4: 2978-2984 (2012).

[197] Socrates G. Infrared and Raman Characteristic Group Frequencies. 3rd ed. England: Wiley\&Sons, 2001.

[198] Bakkour Y, Darcos V, Coumes F, Li S and Coudane J. Brush-like amphiphilic copolymers based on polylactide and 
poly(ethylene glycol): Synthesis, self-assembly and evaluation as drug carrier. Polymer. 54(7): 1746-1754 (2013).

[199] Jung S-H, Song H-Y, Lee $\mathrm{Y}$, Jeong HM and Lee H. Novel Thermoresponsive Polymers Tunable by $\mathrm{pH}$. Macromolecules. 44(6): 1628-1634 (2011).

[200] Darr A and Calabro A. Synthesis and characterization of tyramine-based hyaluronan hydrogels. J. Mater. Sci. Mater. Med. 20(1): 33-44 (2009).

[201] Kobayashi S, Uyama H and Kimura S. Enzymatic Polymerization. Chem. Rev. 101(12): 3793-3818 (2001).

[202] Oudgenoeg G, Hilhorst R, Piersma SR, Boeriu CG, Gruppen H, Hessing M, Voragen AGJ and Laane C. Peroxidase-Mediated Cross-Linking of a Tyrosine-Containing Peptide with Ferulic Acid. J. Agric. Food Chem. 49(5): 2503-2510 (2001).

[203] Ikada Y. Tissue Engineering: Fundamentals and Applications. Oxford, Uk: Elsevier Ltd, 2006.

[204] Meyvis TK, Stubbe BG, Van Steenbergen MJ, Hennink WE, De Smedt SC and Demeester J. A comparison between the use of dynamic mechanical analysis and oscillatory shear rheometry for the characterisation of hydrogels. Int. J. Pharm. 244(1-2): 163-168 (2002).

[205] Kavanagh GM and Ross-Murphy SB. Rheological characterisation of polymer gels. Prog. Polym. Sci. 23(3): 533562 (1998).

[206] Tschoegl NW. The Phenomenological Theory of Linear Viscoelastic Behavior. Berlin, Heidelberg: Springer Berlin Heidelberg, 1989.

[207] Plant GW, Harvey AR and Chirila TV. Axonal growth within poly (2-hydroxyethyl methacrylate) sponges infiltrated with 
Schwann cells and implanted into the lesioned rat optic tract. Brain Res. 671(1): 119-130 (1995).

[208] Civerchia-Perez L, Faris B, LaPointe G, Beldekas J, Leibowitz H and Franzblau C. Use of collagen-hydroxyethylmethacrylate hydrogels for cell growth. Proc. Natl. Acad. Sci. U. S. A. 77(4): 2064-2068 (1980).

[209] Andrés $V$ and Walsh $K$. Myogenin expression, cell cycle withdrawal, and phenotypic differentiation are temporally separable events that precede cell fusion upon myogenesis. $J$. Cell Biol. 132(4): 657-666 (1996).

[210] Rowley JA and Mooney DJ. Alginate type and RGD density control myoblast phenotype. J. Biomed. Mater. Res. 60(2): 217-223 (2002).

[211] Gupta S, Greeshma T, Basu B, Goswami S and Sinha A. Stiffness- and wettability-dependent myoblast cell compatibility of transparent poly(vinyl alcohol) hydrogels. J. Biomed. Mater. Res. Part B Appl. Biomater. 101B(2): 346-354 (2013).

[212] Mawad D, Stewart E, Officer DL, Romeo T, Wagner P, Wagner $\mathrm{K}$ and Wallace GG. A Single Component Conducting Polymer Hydrogel as a Scaffold for Tissue Engineering. Adv. Funct. Mater. 22(13): 2692-2699 (2012).

[213] Salimath AS and García AJ. Biofunctional hydrogels for skeletal muscle constructs. J. Tissue Eng. Regen. Med. (2014). DOI: 10.1002/term.1881

[214] Cooper ST, Maxwell AL, Kizana E, Ghoddusi M, Hardeman EC, Alexander IE, Allen DG and North KN. C2C12 Co-culture on a fibroblast substratum enables sustained survival of contractile, highly differentiated myotubes with peripheral nuclei and adult fast myosin expression. Cell Motil. Cytoskeleton. 58(3): 200-211 (2004). 
[215] Ostrovidov S, Ahadian S, Ramon-Azcon J, Hosseini V, Fujie T, Parthiban SP, Shiku H, Matsue T, Kaji H, Ramalingam M, Bae H and Khademhosseini A. Three-dimensional co-culture of C2C12/PC12 cells improves skeletal muscle tissue formation and function. J. Tissue Eng. Regen. Med. (2014).DOI: 10.1002/term.1956

[216] Wang L-S, Chung JE, Chan PP-Y and Kurisawa M. Injectable biodegradable hydrogels with tunable mechanical properties for the stimulation of neurogenesic differentiation of human mesenchymal stem cells in 3D culture. Biomaterials. 31(6): 1148-1157 (2010).

[217] Fan Z, Zhang $Y$, Fang S, XU C and Li X. Bienzymatically crosslinked gelatin/hyaluronic acid interpenetrating network hydrogels: preparation and characterization. RSC Adv. 5(3): 1929-1936 (2015).

[218] Shu XZ, Liu Y, Luo Y, Roberts MC and Prestwich GD. Disulfide Cross-Linked Hyaluronan Hydrogels. Biomacromolecules. 3(6): 1304-1311 (2002).

[219] Bhat S, Tripathi A and Kumar A. Supermacroprous chitosanagarose-gelatin cryogels: in vitro characterization and in vivo assessment for cartilage tissue engineering. J. $R$. Soc. Interface. 8(57): 540-554 (2011).

[220] Kim J, Park Y, Tae G, Lee KB, Hwang CM, Hwang SJ, Kim IS, Noh $I$ and Sun K. Characterization of low-molecular-weight hyaluronic acid-based hydrogel and differential stem cell responses in the hydrogel microenvironments. J. Biomed. Mater. Res. A. 88(4): 967-975 (2009).

[221] Grover CN, Gwynne JH, Pugh N, Hamaia S, Farndale RW, Best $\mathrm{SM}$ and Cameron RE. Crosslinking and composition influence the surface properties, mechanical stiffness and cell reactivity of collagen-based films. Acta Biomater. 8(8): 3080-3090 (2012). 
[222] Sarker B, Singh R, Silva R, Roether JA, Kaschta J, Detsch R, Schubert DW, Cicha I and Boccaccini AR. Evaluation of fibroblasts adhesion and proliferation on alginate-gelatin crosslinked hydrogel. PLoS One. 9(9): e107952 (2014).

[223] Chung IM, Enemchukwu NO, Khaja SD, Murthy N, Mantalaris A and García AJ. Bioadhesive hydrogel microenvironments to modulate epithelial morphogenesis. Biomaterials. 29(17): 2637-2645 (2008).

[224] Lei Y, Gojgini S, Lam J and Segura T. The spreading, migration and proliferation of mouse mesenchymal stem cells cultured inside hyaluronic acid hydrogels. Biomaterials. 32(1): 39-47 (2011).

[225] Calderon L, Collin E, Velasco-Bayon D, Murphy M, O'Halloran $\mathrm{D}$ and Pandit A. Type II collagen-hyaluronan hydrogel-a step towards a scaffold for intervertebral disc tissue engineering. Eur. Cell. Mater. 20: 134-148 (2010).

[226] Camci-Unal G, Cuttica D, Annabi N, Demarchi D and Khademhosseini A. Synthesis and Characterization of Hybrid Hyaluronic Acid-Gelatin Hydrogels. Biomacromolecules. 14(4): 1085-1092 (2013).

[227] Sahoo S, Chung C, Khetan S and Burdick JA. Hydrolytically Degradable Hyaluronic Acid Hydrogels with Controlled Temporal Structures. Biomacromolecules. 9(4): 1088-1092 (2009).

[228] Pan L, Ren Y, Cui F and Xu Q. Viability and differentiation of neural precursors on hyaluronic acid hydrogel scaffold. J. Neurosci. Res. 87(14): 3207-3220 (2009).

[229] Wang T-W and Spector M. Development of hyaluronic acidbased scaffolds for brain tissue engineering. Acta Biomater. 5(7): 2371-2384 (2009). 
[230] Buxboim A, Ivanovska IL and Discher DE. Matrix elasticity, cytoskeletal forces and physics of the nucleus: how deeply do cells 'feel' outside and in?. J. Cell Sci. 123(3): 297-308 (2010).

[231] Bershadsky AD, Balaban NQ and Geiger B. Adhesiondependent cell mechanosensitivity. Annu. Rev. Cell Dev. Biol. 19: 677-695 (2003).

[232] Oryan A, Alidadi S, Moshiri A and Maffulli N. Bone regenerative medicine: classic options, novel strategies, and future directions. J. Orthop. Surg. Res. 9(1): 18 (2014).

[233] Oh S-A, Lee H-Y, Lee JH, Kim T-H, Jang J-H, Kim H-W and Wall I. Collagen three-dimensional hydrogel matrix carrying basic fibroblast growth factor for the cultivation of mesenchymal stem cells and osteogenic differentiation. Tissue Eng. Part A. 18(9-10): 1087-1100 (2012).

[234] Chargé SBP and Rudnicki MA. Cellular and molecular regulation of muscle regeneration Physiol. Rev. 84(1): 209238 (2004).

[235] Dhawan J and Helfman DM. Modulation of acto-myosin contractility in skeletal muscle myoblasts uncouples growth arrest from differentiation. J. Cell Sci. 117(17): 3735-3748 (2004).

[236] Engler AJ, Sen S, Sweeney HL and Discher DE. Matrix Elasticity Directs Stem Cell Lineage Specification. Cell. 126(4): 677-689 (2006).

[237] Schulz RM and Bader A. Cartilage tissue engineering and bioreactor systems for the cultivation and stimulation of chondrocytes. Eur. Biophys. J. 36(4-5): 539-568 (2007).

[238] Gallego Ferrer G, Salmerón Sánchez M, Gómez Ribelles JL, Romero Colomer FJ and Monleón Pradas M. Nanodomains in a hydrophilic-hydrophobic IPN based on poly(2-hydroxyethyl 
acrylate) and poly(ethyl acrylate). Eur. Polym. J. 43: 31363145 (2007).

[239] Araújo N, Gomes D, Gómez Ribelles JL, Monleón Pradas M and Mano JF. Dynamic-mechanical behavior of hydrophobichydrophilic interpenetrating copolymer networks. Polym. Eng. Sci. 46: 930-937 (2006).

[240] Pérez Olmedilla M, Garcia-Giralt N, Monleón Pradas M, Benito Ruiz P, Gómez Ribelles JL, Cáceres Palou E and Monllau García JC. Response of human chondrocytes to a non-uniform distribution of hydrophilic domains on poly (ethyl acrylate-cohydroxyethyl methacrylate) copolymers. Biomaterials. 27: 1003-1012 (2006).

[241] Gallego Ferrer G, Soria Meliá JM, Hernández Canales J, Meseguer Dueñas JM, Romero Colomer F, Monleón Pradas M, Gómez Ribelles JL, Pissis P and Polizos G. Poly(2-hydroxyethyl acrylate) hydrogel confined in a hydrophobous porous matrix. Colloid Polym. Sci. 283: 681-690 (2004).

[242] Stathopoulos A, Klonos P, Kyritsis A, Pissis P, Christodoulides C, Rodriguez Hernández JC, Monleón Pradas $M$ and Gómez Ribelles JL. Water sorption and polymer dynamics in hybrid poly(2-hydroxyethyl-co-ethyl acrylate)/silica hydrogels. Eur. Polym. J. 46(1): 101-111 (2010).

[243] Gallego Ferrer G, Monleón Pradas M, Gómez Ribelles JL, Romero Colomer F, Castilla-Cortázar I and Vidaurre A. Influence of the nature of the porous confining network on the sorption, diffusion and mechanical properties of hydrogel IPNs. Eur. Polym. J. 46(4): 774-782 (2010).

[244] Gallego Ferrer G, Monleón Pradas M, Gómez Ribelles JL and Pissis P. Swelling and thermally stimulated depolarization currents in hydrogels formed by interpenetrating polymer networks. J. Non. Cryst. Solids. 235-237: 692-696 (1998). 
[245] Kyritsis A, Pissis P, Gómez Ribelles JL and Monleón Pradas M. Polymer-water interactions in poly ( hydroxyethy1 acrylate ) hydrogels studied by dielectric, calorimetric and sorption isotherm measurements. Polym. Gels Networks. 3: 445-469 (1995).

[246] Greenspan L. Humidity fixed points of Binary Saturated Solutions. J Res Nat Bur Stand A Phys Chem. 81A: 89-96 (1977).

[247] DiSilvestro MR and Suh J-KF. A cross-validation of the biphasic poroviscoelastic model of articular cartilage in unconfined compression, indentation, and confined compression. J. Biomech. 34: 519-525 (2001).

[248] DiSilvestro MR, Zhu Q and Suh J-KF. Biphasic Poroviscoelastic Simulation of the Unconfined Compression of Articular Cartilage: II-Effect of Variable Strain Rates. J. Biomech. Eng. 123(2): 198 (2001).

[249] Acosta Santamaría VA, García Aznar JM, Ochoa I and Doblare M. Effect of Sample Pre-Contact on the Experimental Evaluation of Cartilage Mechanical Properties. Exp. Mech. 53: 911-917 (2013).

[250] Bursać PM, Obitz TW, Eisenberg SR and Stamenović D. Confined and unconfined stress relaxation of cartilage: appropriateness of a transversely isotropic analysis. J. Biomech. 32: 1125-1130 (1999).

[251] Hung CT, Mauck RL, Wang CCB, Lima EG and Ateshian GA. A paradigm for functional tissue engineering of articular cartilage via applied physiologic deformational loading. Ann. Biomed. Eng. 32: 35-49 (2004).

[252] Wilson W, van Donkelaar CC, van Rietbergen B and Huiskes R. A fibril-reinforced poroviscoelastic swelling model for articular cartilage. J. Biomech. 38: 1195-1204 (2005). 
[253] Korhonen RK, Laasanen MS, Töyräs J, Rieppo J, Hirvonen J, Helminen $\mathrm{HJ}$ and Jurvelin JS. Comparison of the equilibrium response of articular cartilage in unconfined compression, confined compression and indentation. J. Biomech. 35: 903909 (2002).

[254] Schinagl RM, Gurskis D, Chen AC and Sah RL. Depthdependent confined compression modulus of full-thickness bovine articular cartilage. J. Orthop. Res. 15(4): 499-506 (1997).

[255] Acosta Santamaría V, Deplaine H, Mariggió D, VillanuevaMolines AR, García-Aznar JM, Gómez Ribelles JL, Doblaré M, Gallego Ferrer $G$ and Ochoa I. Influence of the macro and micro-porous structure on the mechanical behavior of poly(Ilactic acid) scaffolds. J. Non. Cryst. Solids. 358: 3141-3149 (2012).

[256] JMansour JM and Mow VC. The permeability of articular cartilage under compressive strain and at high pressures. J. Bone Joint Surg. Am. 58(4): 509-516 (1976).

[257] Monleón Pradas $M$, Gómez Ribelles JL, Serrano Aroca A, Gallego Ferrer G, Suay Antón J and Pissis P. Porous poly(2hydroxyethyl acrylate) hydrogels. Polymer. 42(10): 4667-4674 (2001).

[258] Serrano Aroca A, Campillo Fernández AJ, Gómez Ribelles JL, Monleón Pradas M, Gallego Ferrer $G$ and Pissis P. Porous poly(2-hydroxyethyl acrylate) hydrogels prepared by radical polymerisation with methanol as diluent. Polymer. 45(26): 8949-8955 (2004).

[259] Gallego Ferrer G, Monleón Pradas M, Gómez Ribelles JL and Salmerón Sánchez M. Thermodynamical analysis of the hydrogel state in poly(2-hydroxyethyl acrylate). Polymer. 45(18): 6207-6217 (2004). 
[260] Gallego Ferrer G, Monleón Pradas M and Gómez Ribelles JL. Thermodynamics of water sorption in acrylic homonetworks and IPNs. Macromol. Symp. 200(1): 217-226 (2003).

[261] Carter DR and Wong M. Modelling cartilage mechanobiology. Phil. Trans. R. Soc. Lond. B. 358: 1461-1471 (2003).

[262] Fung YC. Biomechanics: mechanical properties of living tissues. J Boimech. 38: 483-490 (1993).

[263] Thambyah A, Nather A and Goh J. Mechanical properties of articular cartilage covered by the meniscus. Osteoarthr. Cartil. 14: 580-588 (2006).

[264] Boschetti F, Miotti C, Massi F, Colombo M, Quaglini V, Peretti GM and Pietrabissa R. Second Joint EMBS-BMES Conference 2002 - Engineering in Medicine and Biology Society Annual Fall Meeting of the Biomedical Engineering Society. Proceedings of the Second Joint 24th Annual Conference and the Annual Fall Meeting of the Biomedical Engineering Society. Engineering in Medicine and Biology. 3: 2581-2582 (2002).

[265] Sperling LH. Interpenetrating Polymer Networks: An Overview. Washington DC: American Chemical Society, 1994.

[266] Salmerón Sánchez M, Gallego Ferrer G, Torregrosa Cabanilles C, Meseguer Dueñas JM, Monleón Pradas M and Gómez Ribelles JL. Forced compatibility in poly(methyl acrylate)/poly(methyl methacrylate) sequential interpenetrating polymer networks. Polymer. 42: 1007110075 (2001).

[267] Zhao C, Xu M, Zhu $W$ and Luo X. Novel interpenetrating polymer networks of polypropylene/poly(n-butyl acrylate). Polymer. 39(2): 275-281 (1998).

[268] Stathopoulos AT, Kyritsis A, Gallego Ferrer G, Gómez Ribelles IL, Christodoulides C and Pissis P. Cooperative Segmental Motions in Ethyl Acrylate/Triethylene Glycol Dimethacrylate 
Copolymer Networks Studied by Dielectric Techniques. Macromolecules. 44(20): 8233-8244 (2011).

[269] U. Gedde, Polymer Physics, 1st ed. London: Chapman\&Hall, 1995. 


\section{Glossary}

${ }^{1}$ H-NMR: Proton Nuclear Magnetic Resonance

$\mathrm{ACl}$ : autologous chondrocyte implantation

ADAMTS: A Desintegrin And Metalloproteinase with Thrombospondin motifs

AR: aspect ratio

ATR: attenuated total reflectance

bFGF: basic Fibroblast Growth Factor

BHT: 2,6-Di-tert-butyl-4-methylphenol

BM-hMSCs: human bone marrow mesenchymal stem cells

BMPs: bone morphogenetic proteins

BP: benzophenone

BSA: bovine serum albumin

CC: confined compression

CF-KRB: Calcium Free Kebs Ringer Buffer

$\mathrm{Cl}$ : contractility inhibitor

$\mathrm{CM}$ : chondrogenic media

CNWs: cellulose nanowhiskers

$\mathrm{CO}_{2}$ : carbon dioxide

COMP: cartilage oligomeric matrix protein

$\mathrm{COOH}$ : carboxylic groups

CS: chondroitin sulfate

$D$ : diffusion coefficient

$\mathrm{D}_{2} \mathrm{O}$ : deuterium oxide

DAPI: 4',6-diamidino-2-phenylindole 
DM: differentiation medium

DMEM: Dulbecco's Modified Eagle Medium

DMS: Dynamic Mechanical Spectroscopy

DMSO-d6: Dimethyl sulfoxide-d6

DPBS: Dulbecco's phosphate buffered saline

DS: dynamic shear

DSC: Differential Scanning Calorimetry

$E$ or $E_{s}$ : Young's modulus

$E^{\prime}$ : Young's storage modulus

EA: ethyl acrylate

ECM: Extracellular Matrix

EDC: 1-ethyl-3-(3-dimethyl aminopropyl) carbodiimide

EGDMA: ethylene glycol dimethacrylate

ES: equilibrium shear

EthD-1: ethidium homodimer-1

EWC: Equilibrium Water Content

EWC': Equilibrium Water Content referred to the hydrophilic component

$E W C_{r}$ : reabsorption Equilibrium Water Content

$E W C_{r}^{\prime}$ : reabsorption Equilibrium Water Content referred to the hydrophilic component

F: Force

FBS: fetal bovine serum

FGF: fibroblast growth factor

FTIR: Fourier Transformed Infrared Spectroscopy

$\mathrm{G}^{\prime}$ : shear storage modulus 
$\mathrm{G}^{\prime \prime}$ : shear loss modulus

$\left|G^{*}\right|$ : the complex modulus magnitude

GAGs: glycosaminoglycans

GEL or GEL-TA: tyramine grafted gelatin hydrogel

GEL-GTA: glutaraldehyde crosslinked gelatin

GEL-Tyr: tyramine grafted gelatin

GEL-TA xPLLA-ES: tyramine grafted gelatin composites with $x$ percentage of PLLA-ES fibers

GEL-TA xPLLA-HT: tyramine grafted gelatin composites with $x$ percentage of PLLA-HT fibers

GLA protein: $\gamma$-carboxyglutamic acid-rich protein

GM: growth medium

GMP: Good Manufacturing Practices

GTA: glutaraldehyde

Gly: glycine

$\mathrm{H}_{2} \mathrm{O}$ : water

$\mathrm{H}_{2} \mathrm{O}_{2}$ : hydrogen peroxide

$H_{a}$ : aggregated modulus

HA: hyaluronic acid

HA-Tyr: tyramine grafted hyaluronic acid

HEA: 2-hydroxyethyl acrylate

HEMA: 2-hydroxyethyl methacrylate

HEPES: 4-(2-hydroxyethyl)piperazine-1-ethanesulphonic acid

hMSCs: human mesenchymal stem cells

HMW: high molecular weight

HRP: horseradish peroxidase 
HUVECS: human umbilical cord vein endothelial cells

I: indentation

IGFs: insulin growth factors

IL-1: interleukin 1

IPNs: interpenetrated polymer networks

$k$ : permeability

KRB: Krebs Ringer Buffer

KS: keratan sulfate

LMW: low molecular weight

$\mathrm{MACl}$ : Matrix-induced ACl

MES: 2-(N-Morpholino)ethanesulfonic acid

MMPs: matrix metalloproteinases

MSCs: mesenchymal stem cells

MTS:(3-(4,5-dimethylthiazol-2-yl)-5-(3-carboxymethoxyphenyl)-2-(4-

sulfophenyl)-2H-tetrazolium)

MWCO: molecular weight cut off

NHS: N-hydroxysuccinimide

OA: osteoarthritis

OATS: Osteochondral autografting

OCT: Optimal Cutting Temperature compound

PBS: phosphate-buffered saline

PDI: polydispersity index

PEA: poly(ethyl acrylate)

PEG: Poly(ethylene glycol)

Ph: phenol 
PHEA: poly(2-hydroxyethyl acrylate)

PHEMA: poly(2-hydroxyethyl methacrylate)

PLLA: poly(L-lactic acid)

PLLA-BP: PLLA fibers activated with BP

PLLA-ES: PLLA electrospun fibers

PLLA-HT: PLLA high turbulent flow microfibers

PLLA-PHEMA: PLLA microfibers grafted with PHEMA

Pro: proline

RA: rheumatoid arthritis

$\mathrm{RH}$ : relative humidity

ROM: rule of mixtures

SEC: Size exclusion chromatography

SEM: Scanning Electron Microscope

t: time

TCSR: tensile constant strain rate

TEGMA: triethylenglycol dimethacrylate

$T_{g}$ : glass transition temperature

TGF- $\beta 1$ : transforming growth factor beta-1

TGF- $\beta 2$ : transforming growth factor beta-2

TNF- $\alpha$ : tumor necrosis factor-alpha

TSG-6: tumor necrosis factor-stimulated gene 6 protein

TSR: tensile stress relaxation

Tyr: tyramine

UC: unconfined compression

$w$ : water content 
$w^{\prime}:$ water content referred to the hydrophilic component

$w_{r}$ : reabsorption water content

$w_{r}^{\prime}$ : reabsorption water content referred to the hydrophilic component

Wnt: Wingless family 


\section{Curriculum Vitae}

\section{Education}

(2005-2011) Chemical Engineer.

University: Universitat Politècnica de València. Valencia (Spain)

(2011-2015) PhD studies in "Technologies for Health and Welfare" at Universitat Politècnica de València with the economic support from the Spanish Ministry of Economy and Competitiveness through the FPI BES-2011-046144 PhD grant.

Thesis title: "Protein-based injectable hydrogels towards the regeneration of articular cartilage". Supervisor: Gloria Gallego Ferrer.

\section{Research stays}

2013- Research stay (EEBB 2013-MEC) in the Department of Fibre and Polymer Technology, Royal Institute of Technology, KTH, Stockholm (Sweden) for four months. Supervisor: Ulrica Edlund.

2014- Research stay (EEBB 2014-MEC) in the Department of Biomedical Engineering, University of Glasgow (United Kingdom) for four months. Supervisor: Manuel Salmerón Sánchez.

\section{Publications}

[1] A. Vallés-Lluch, S. Poveda-Reyes, P. Amorós, D. Beltrán and M. Monleón Pradas. Hyaluronic Acid-Silica Nanohybrid Gels. Biomacromolecules. 14: 4217-4225 (2013)

[2] S. Manzano, S. Poveda-Reyes, G. Gallego Ferrer, I. Ochoa and M. $\mathrm{H}$. Doweidar. Computational analysis of cartilage implants based on an interpenetrated polymer network for tissue repairing. Computer Methods and Programs in Biomedicine. 116: 249-259 (2014)

[3] S. Poveda-Reves, T. C. Gamboa-Martínez, S. Manzano, I Ochoa, M. H. Doweidar, J. L. Gómez Ribelles and G. Gallego Ferrer. Engineering Interpenetrating Polymer Networks of Poly(2-Hydroxyethyl 
Acrylate) as Ex Vivo Platforms for Articular Cartilage Regeneration. International Journal of Polymeric Materials and Polymeric Biomaterials. 64 (14): 745-754 (2015).

[4] S. Poveda-Reyes, L. R. Mellera-Oglialoro, R. Martínez-Haya, T. C. Gamboa-Martínez, J. L. Gómez Ribelles and G. Gallego Ferrer. Reinforcing an Injectable Gelatin Hydrogel with PLLA Microfibers: Two Routes for Short Fiber Production. Macromolecular Materials and Engineering. 300(10): 977-988 (2015).

[5] S. Poveda-Reyes, A. Rodrigo-Navarro, T. C. Gamboa-Martínez, J. C. Rodríguez-Cabello, L. Quintanilla-Sierra, U. Edlund and G. Gallego Ferrer. Injectable Composites of Loose Microfibers and Gelatin with Improved Interfacial Interaction for Soft Tissue Engineering. Polymer. 74: 224-234 (2015).

[6] S. Poveda-Reyes, E. Sanmartín-Masiá and G. Gallego Ferrer. Extracellular matrix-inspired gelatin/hyaluronic acid injectable hydrogels. (In process).

[7] S. Poveda-Reyes, V. Moulisova, E. Sanmartín-Masiá, M. SalmerónSánchez and G. Gallego Ferrer. Myogenic Differentiation in GelatinHyaluronic Acid Injectable Hydrogels. (Submitted to Macromolecular Bioscience).

[8] S. Poveda-Reyes, V. Moulisova, E. Sanmartín-Masiá, L. QuintanillaSierra, M. Salmerón-Sánchez and G. Gallego Ferrer. GelatinHyaluronic Acid Hydrogels promote BM-hMSCs differentiation towards cartilage phenotype. (In process).

\section{Conferences}

[1] 25th European Conference on Biomaterials (ESB 2013)

Authors: Leonardo Rubén Mellera-Oglialoro, Tatiana Carolina Gamboa-Martínez, Sara Poveda-Reyes, José Luis Gómez Ribelles, Gloria Gallego Ferrer.

Title: Gelatin Gels Reinforced with Polymeric Microfibers for Tissue Engineering 
Type of participation: Oral communication

Publication: ISBN 978-84-695-7831-5

Place: Madrid Date: 8-12th September 2013

\section{[2] 25th European Conference on Biomaterials (ESB 2013)}

Authors: Sara Poveda-Reyes, Tatiana Carolina Gamboa-Martínez, Carlos Pelegay Salas, Mohamed Hamdy Doweidar, José Luis Gómez Ribelles, Ignacio Ochoa Garrido, Gloria Gallego Ferrer.

Title: Interpenetrated Polymer Networks as Host Tissue in a Bioreactor for Articular Cartilage Regeneration

Type of participation: Poster

Publication: ISBN 978-84-695-7831-5

Place: Madrid Date: 8-12th September 2013

\section{[3] I Congreso Biomedicina Predocs}

Authors: Sara Poveda-Reyes, Esther Sanmartín-Masiá, Petra CantóLoras, Félix González-Fernández, Gloria gallego Ferrer.

Title: Hidrogeles inyectables de gelatina y ácido hialurónico para la regeneración del cartílago articular: dos rutas de combinación.

Type of participation: Poster

Place: Valencia Date: 28th November 2014

[4] 5th International Symposium Interface Biology of Implants (IBI)

Authors: Sara Poveda-Reyes, Vladimira Moulisova, Esther SanmartínMasiá, Manuel Salmerón-Sánchez, Gloria Gallego-Ferrer.

Title: Injectable extracellular matrix-inspired gelatin/hyaluronic acid hydrogels for soft tissue engineering

Type of participation: Poster

Place: Rostock (Germany) Date: 6-8th May 2015. 


\section{[5] XLII Annual ESAO Conference (ESAO 2015)}

Authors: Sara Poveda-Reyes, Aleixandre Rodrigo-Navarro, Tatiana C. Gamboa-Martínez, José C. Rodíguez-Cabello, Luis Quintanilla-Sierra, Ulrica Edlund, Gloria Gallego Ferrer.

Title: Injectable Composites of Loose Microfibers and Gelatin for Soft Tissue Engineering

Type of participation: Oral communication

Place: Leuven (Belgium) Date: 2-5th September 2015

\section{I+D+i Projects}

[1] Title: Diseño y fabricación de una plataforma biomimética tipo scaffold/soporte para la regeneración del cartílago articular (DPI2010-20399-C04-03)

Funding: Spanish Ministry of Science and Innovation

Dates: 2011-2013

Principal researcher: Gloria Gallego Ferrer

[2] Title: Estimulación mecánica local de células mesenquimales de cara a su diferenciación osteogénica y condrogénica en medicina regenerativa (MINECO, MAT2013-46467-C4-1-R)

Funding: Spanish Ministry of Economy and Competitiveness

Dates: 2014-2016

Principal researchers: José Luis Gómez Ribelles and Gloria Gallego Ferrer (co-IPs)

\section{Courses}

- Prevention of occupational hazards (Laboratories/Research. July 2014)

- 6th Summer School on Medicines (SSM6) Barcelona (6-11th July 2014) 


\section{Grants}

- Doctoral grant: FPI grant BES-2011-046144 from the Spanish Ministry of Economy and Competitiveness for the development of my PhD studies.

- FPI fellowships: EEBB-I-13-06179 and EEBB-I-14-08725 research stay grants. Thanks to these grants I could develop my scientific skills in the KTH (Stockholm, Sweden) and University of Glasgow (Glasgow, United Kingdom).

\section{Supplementary information}

- Software, user level: Windows XP/Vista/7, Word, Excel, Powerpoint, Project, MathCad, ChemCad, AutoCad, ALOHA, Menfis, Statgraphics.

- Medium knowledge in CAD software such as ProEngineer, Inventor...

- Council member of the Department of Applied Thermodynamics (Universitat Politècnica de València) as student representative (2012/2013 and 2013/2014).

- Tutor of Final Degree Projects in the Chemical Engineer Degree. Universitat Politècnica de València (UPV).

- Teaching: 7.25 credits $(72,5 \mathrm{~h})$ Department of Applied Thermodynamics. UPV.

- Preparation and writing of European funding proposals (MSCA-ITN and FET) (2015-2016).

- Driver license: $\mathrm{B}$. 
